DOE/RL-95-82

Revision 0

UC-630

\title{
Inventory of Miscellaneous Streams
}

Date Published

September 1995

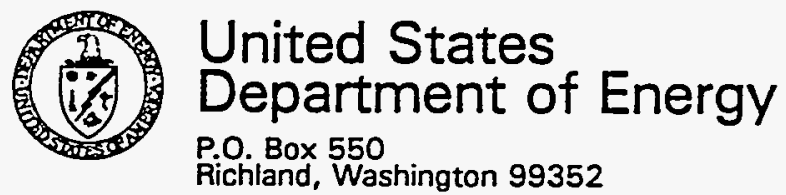

Richland, Washington 99352

Approved for Public Release

DISTRIBUTION OF THS DOCUMENT IS UNLAMTED 


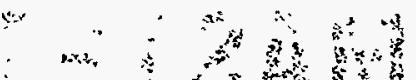




\section{DISCLAIMER}

Portions of this document may be illegible in electronic image products. Images are produced from the best available original document. 


\section{TABLE OF CONTENTS}

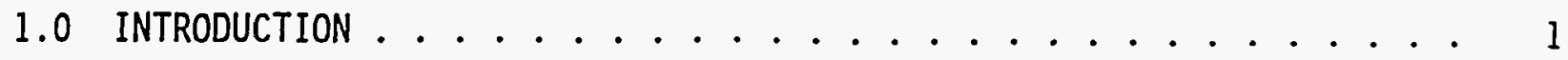

2.0 HANFORD SITE DESCRIPTION ................... 1

3.0 MISCELLANEOUS STREAM INVENTORY . . ........... 3

3.1 Data Explanation ................. 3

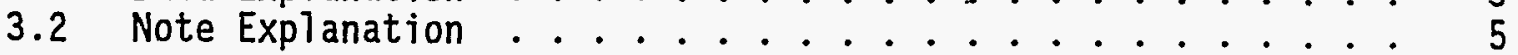

4.0 SOURCE WATER DESCRIPTION . . . . . . . . ..... 6

4.1 Surface Water from the Columbia River. . . . . . . . 6

4.2 Sanitary Water .............. 6

4.3 Groundwater (We11 Water) ................ 7

4.4 Storm Water.................. 7

5.0 PROCESS DESCRIPTION ................... . . . 8

5.1 Surface Water Process Description . . . . . . . . . . 8

5.2 Sanitary Water Process Descriptions . . . . . . . . . 8

5.2 .1 Steam Condensate . . . . . . . . . 8

5.2 .2 Cooling Water ............. 8

5.2 .3 sink Drains . . . . . . . . . . . . 9

5.3 Groundwater Process Description . . . . . . . . . . 9

5.4 Storm Water Process Description . . . . . . . . . 9

6.0 REFERENCES .......................... 75

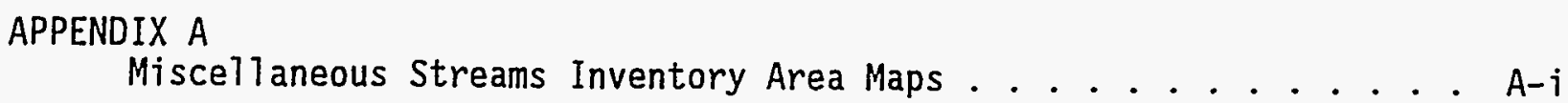

Figure and Table

Figure 2-1. Hanford Site. ............... 2

Table 3-1. Miscellaneous Stream Inventory........... 11

\section{DISCLAIMER}

This report was prepared as an account of work sponsored by an agency of the United States Government. Neither the United States Government nor any agency thereof, nor any of their employees, makes any warranty, express or implied, or assumes any legal liability or responsibility for the accuracy, completeness, or usefulness of any information, apparatus, product, or process disclosed, or represents that its use would not infringe privately owned rights. Reference herein to any specific commercial product, process, or service by trade name, trademark, manufacturer, or otherwise does not necessarily constitute or imply its endorsement, recommendation, or favoring by the United States Government or any agency thereof. The views and opinions of authors expressed herein do not necessarily state or reflect those of the United States Government or any agency thereof. 
DOE/RL-95-82, Rev. 0
$09 / 95$

(This page intentionally left blank) 


\section{INVENTORY OF MISCELLANEOUS STREAMS}

\subsection{INTRODUCTION}

On December 23, 1991, the U.S. Department of Energy, Rich] and Operations Office (RL) and the Washington State Department of Ecology (Ecology) agreed to adhere to the provisions of the Department of Ecology Consent Order No. DE 91NM-177 (Consent Order) (Ecology and U.S. DOE) 1991). The Consent Order lists the regulatory milestones for liquid effluent streams at the Hanford Site to comply with the permitting requirements of Washington Administrative Code (WAC) 173-216 (State Waste Discharge Permit Program) or WAC 173-218 (Washington Underground Injection Control Program) where applicable.

The RL provided the U.S Congress a Plan and Schedule to discontinue disposal of contaminated liquid effluent into the soil column on the Hanford Site (DOE 1987). The plan and schedule document contained a strategy for the implementation of alternative treatment and disposal systems. This strategy included prioritizing the streams into two phases. The Phase I streams were considered to be higher priority than the Phase II streams. The actions recommended for the Phase I and II streams in the two reports were incorporated in the Hanford Federal Facility Agreement and Consent Order (Tri Party Agreement) (Ecology, et al. 1994). Miscellaneous Streams are those liquid effluents streams identified within the Consent Order that are discharged to the ground but are not categorized as Phase I or Phase II Streams.

Miscellaneous streams discharging to the soil column on the Hanford Site are subject to requirements of several milestones identified in the Consent Order. The "Plan and Schedule for Disposition and Regulatory Compliance for -Misce 71 aneous Streams" (DOE/RL-93-94, Rev. 1) provides a $p 7$ an and schedule for the disposition of Miscellaneous Streams to satisfy one of the Consent Order requirements. One of the commitments (Activity 6-2.2) established in the plan and schedule is to annually update the Miscellaneous Stream Inventory. The annual update will continue until September of 1998, at which time the four categorical permit applications are scheduled to have been submitted.

\subsection{HANFORD SITE DESCRIPTION}

The Hanford Site covers approximately 1,450 square kilometers $(560$ square miles) of semiarid 7 and that is owned by the U.S. Government and managed by DOE-RL. The Hanford Site is located northwest of the city of Richland, Washington (Figure 2-1). The city of Richland adjoins the southeastern most portion of the Hanford Site boundary and is the nearest population center.

Activities on the Hanford Site are centralized in numerically designated areas. The 100 Areas, located along the Columbia River, contains deactivated 
DOE/RL-95-82, Rev. 0

$09 / 95$

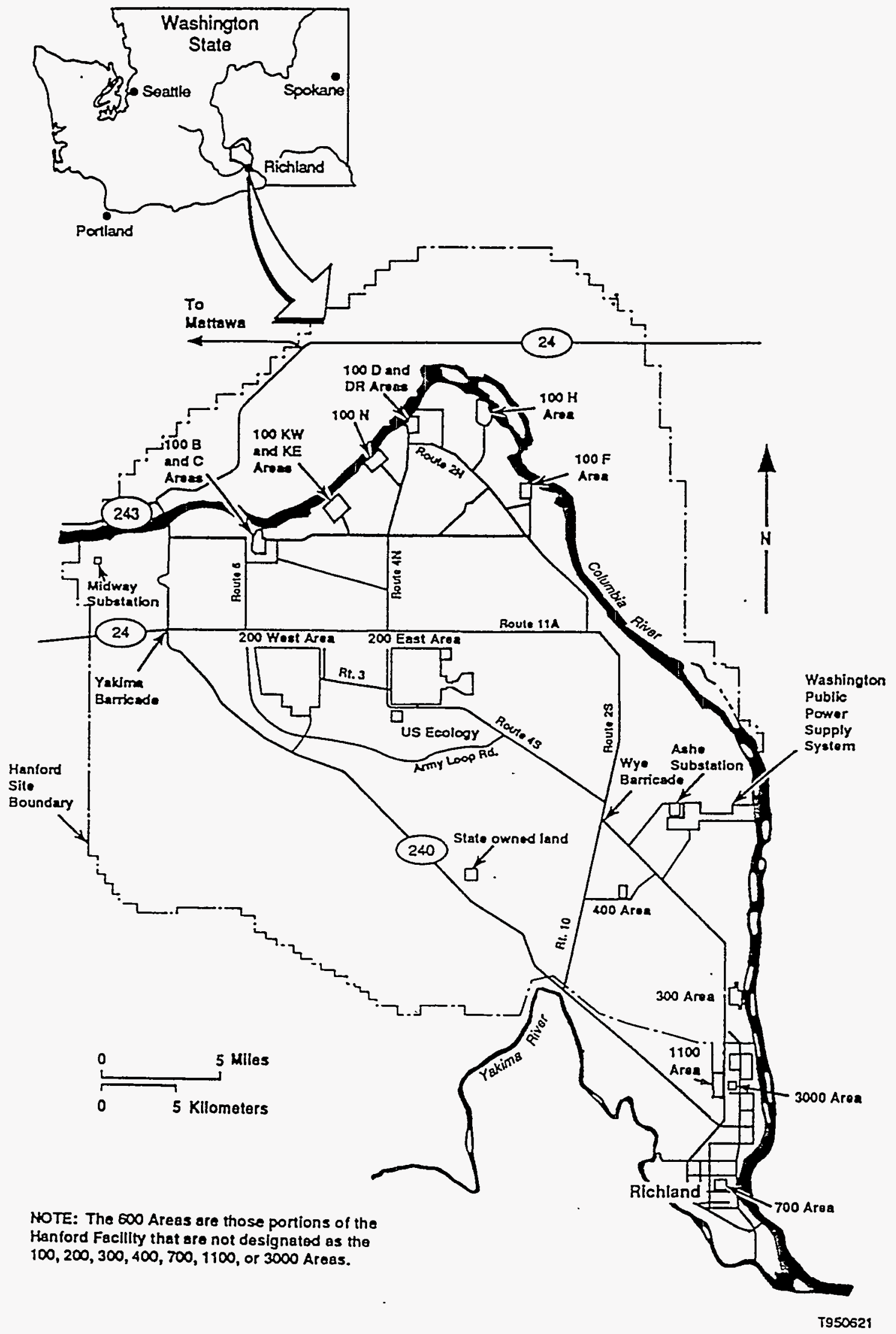

Figure 2-1. Hanford Site 
reactors. The processing units are located in the 200 Areas, which are on a plateau approximately 11 kilometers (7 miles) from the Columbia River. The 300 Area, located adjacent to and north of Richland, contains research and development laboratories. The 400 Area, 8 kilometers (5 miles) northwest of the 300 Area, contains the Fast Flux Facility previously used for testing liquid metal reactor systems. The 600 Area covers all locations not specifically given an area designation. Adjacent to the north of Richland, the 1100 Area contains offices associated with administration, maintenance, transportation, and materials procurement and distribution. The 3000 Area, located between the 1100 Area and 300 Area, contains engineering offices and administrative offices. Additional administrative offices are also located in the 700 Area.

The Miscellaneous Stream inventory lists disposal site locations limited to activities conducted by DOE-RL on the Hanford Site, and excludes activities conducted by others on lands covered by leases, use permits, easements and other agreements whereby 1 and is used by parties other than DOE-RL. For example, the Miscellaneous Stream inventory does not cover activities on state owned or leased lands, lands owned or under use agreements by the Bonnevilie Power Administration, and Tands Teased to the Washington Public Power Supply System.

\subsection{MISCELLANEOUS STREAM INVENTORY}

The inventory of Miscellaneous Streams, given in Table 3-1, identifies 1iquid effluent discharges from a fixed location to an engineered disposal structure at a measurable flowrate. An engineered disposal structure, as defined in the Plan and Schedule, is a man-made structure that aids in infiltration of fluids into the soil. Appendix $A$ of this inventory, contains detailed area maps of the 100,200,300, and 400 Areas that identify the locations of the active miscellaneous streams disposal sites. The stream identification numbers on the maps, corresponds to the "Stream \#" field on the inventory (Table 3-1). Those Miscellaneous Streams that are identified in Table 4 of the Consent Order have been permitted through other means and are not included in this inventory.

\subsection{Data Explanation}

Information on each column of the inventory is described in this section. Inactive streams were only included if they collect storm water from an area surrounding the disposal structure. There are a few disposal structure that are planned to be reactivated as stated in the comments section.

\section{Area}

This information corresponds with where the disposal structure is in regards to Hanford Areas (e.g., 200E, 100N, etc). 


\section{Process Description}

The process description should contain the building number and process generating the stream (e.g., steam condensate). Detailed process descriptions are described in Section 5.0 .

\section{Source Water}

Source water corresponds to the key at the bottom of the each page of the inventory. If there is more than one stream discharging to the disposal structure all source waters should be identified. Detailed descriptions of the four types of source water are described in Section 4.0 .

\section{Stream \#}

The stream number is a consecutive reference ID\#. When a stream is eliminated or rerouted, it is noted in the comments but the stream \# is not reused. Some streams on the initial inventory did not belong on the updated inventory (e.g., when a stream was permitted during a previous action). These streams were removed and their ID\#s were reused for newly added streams. This eliminated the process of renumbering streams and allowed for a historical file to be kept.

\section{Disposal Structure}

Originally, this field was used to identify different types of disposal structures. As streams were submitted for addition to the inventory, it became clear that definitions of different disposal structures were not being used consistently. This inconsistency made this field of little use. However, this field does identify different disposal structures for the non-injection well discharges.

Note

This note field corresponds to the footnote on the original miscellaneous stream inventory. Some of the footnotes were no longer needed (i.e., discharges to 009) and were removed. The definition of each note is described at the bottom on each page of the inventory. Detailed descriptions of each note are described in Section 3.2.

\section{Well Code}

Ecology requested that EPA Well Codes be assigned to the active Class $V$ injection wells. The codes used were:

1. 5 W20 - Industrial Process Water and Waste Disposal Wells

2. 5 Al9 - Cooling Water Return Flow Wells

3. 502 - Stormwater Drainage Wells

4. 504 - Industrial Drainage Wells

\section{Coordinates}

Disposal structure coordinates are identified by Lambert coordinate system.

Flow (gpm)

Flow rates are estimated. Flow rates for each disposal structure are averaged over one-year period in gallons per minute (gpm). 
Comments

This field may contain a variety of information. It contains stream location descriptions (e.g., west side of PUREX), and information on whether a stream. has been eliminated, added, or corrected.

216

This field identifies whether or not the stream is subject to permitting requirements under WAC 173-216. Current7y, storm water discharges are marked "yes" and steam condensate are marked "no" due to agreements with Ecology. Permitting of steam condensate discharges has been deferred indefinitely. If the stream has been eliminated or does not require a permit through current agreement with Ecology, 216 is marked "no".

218

This field identifies whether or not the stream is discharging to an injection well. If the discharge to an injection well has been eliminated, 218 is marked "no".

\subsection{Note Explanation}

There are four possible notes that may be associated with each stream on the injection well registration. These notes may be assigned to each stream as applicable.

$a=$ This note is obsolete.

$b=$ Stream is discharging to an injection well within a surface contaminated area.

$c=$ Potentially contaminated stream.

$\mathrm{d}=$ Disposal site within 300 feet of an active/inactive crib, ditch or trench.

Note "a": This note is obsolete. This note was helpful in assisting permit applicants as to which streams to include in the permit application. Streams have been verified as eliminated, rerouted, or no change.

Note " $b$ ": Stream is discharging to a disposal site within a surface contaminated area. Surface contaminated areas are defined as those near-surface soils contaminated with dangerous and/or radioactive waste. There is a potential for migration of existing contaminants present in the soil of the discharge site to the ground water: Underground injection wells discharging directly to a surface contaminated area are a concern.

The boundaries of surface contaminated areas are often set as a conservative boundary for convenient administrative control, and may also include uncontaminated areas. For example, if there are two surface contamination areas close to each other, a boundary will be set to include both surface contamination areas. 
DOE/RL-95-82, Rev. 0

$09 / 95$

Therefore, this example boundary includes uncontaminated areas in between the surface contamination area.

Note "c": Potentially contaminated stream. Streams are considered potentially contaminated if there is a possibility for contaminants described in WAC 173-200 to enter the source water and cause ground water criteria to be exceeded.

Miscellaneous Streams originating from sources with physical and/or administrative barriers to prevent contaminants from entering the stream are not considered to have a potential for contaminants. Ground water, surface water, or potable waste water, unaltered except for temperature (e.g., steam condensate, uncontaminated storm water, noncontact cooling water), are not considered to have the potential for contaminants exceeding Timits in WAC 173-200.

Note "d": Disposal site within 91-meters (300 feet) of an active/inactive crib, ditch or trench. Cribs, ditches, and trenches were used for the disposal of radioactive contaminants. There is a potential for migration of existing contaminants present in the soil within a 91-meter ( 300 foot) radius of the discharge point. The 91-meter ( 300 foot) criterion has been used as a minimum separation distance for siting new cribs on the Hanford Site. It is considered a conservative distance based on collective experience from borehole dritling in the vicinity of liquid effluent disposal sites. Lateral spreading from adjacent 7 iquid disposal sites greater than 91 meters (300 feet) apart has not been observed to impact either disposal stream.

\subsection{SOURCE WATER DESCRIPTION}

There are four types of source water possible for waste streams on the Hanford Site: surface water from the Columbia River; potable water, groundwater, and storm water.

\subsection{Surface Water from the Columbia River}

Surface water from the Columbia River is pumped from the 100-B, 100-D, or 300 Areas. This water, also called raw water, is filtered to remove large debris, but has not been through any other treatment process.

\subsection{Sanitary Water}

A three-step process converts raw water from the Columbia River into sanitary water, also known as potable water. Equipment used to perform this process includes a stainless steel mixing tank, coagulator settling basins, and multimedia gravity filters. 
In the first step of the treatment process, raw water from the columbia River is supplied to the mixing tank. Chlorine gas, primarily used to control algae and odor, is added to the raw water as it enters the mixing tank.

Water flows from the mixing tank to one of the coagulator settling basins. Aluminum sulfate (alum) is added in the coagulator settling basins as needed. Alum acts as a coagulant, causing flocculate particles to grow in size and density, or agglomerate, and ultimately precipitate to the bottom of the coagulator settling basin.

In the final step, multimedia gravity filters are used to remove the remaining fine particles. The multimedia gravity filters consist of multiple layers of sand, charcoal, and gravel. Gravitational force allows water to pass through gravity filters, causing the finer particles to adhere to the sand grains, charcoal grains, or gravel. Praestol 2525TR ${ }^{1}$ (anionic acrylamide co polymer) is added to water in the gravity filters to aide infiltration of the water in the multimedia gravity filters in the 300 Area Treatment facility only. Chlorine may be added to the potable water in the clearwell to maintain a concentration of one part per million.

The source of sanitary water to the 1100 Area is from the City of Richland. However, the City of Richland sanitary water will not be discussed since storm water is the only source water from a Miscellaneous Stream in the 1100 Area.

\subsection{Groundwater (We11 Water)}

Groundwater is used as the primary source water only in the 400 Area. Three deep wells (one primary and two backup) supply water to three storage tanks. The water is chlorinated with a $1 \%$ sodium hypochlorite solution prior to entering the storage tanks. Storage tank water is used as supply water. The sanitary water is pumped throughout the 400 Area for domestic and process use.

Numerous backup wells also exist on the Hanford Site. However, these wells are only used in case of an emergency.

\subsection{Storm Water}

Storm water is rain fall and snow melt run-off.

1 Praestol 2515TR is a Grace Deerborn product. 
DOE/RL-95-82, Rev. 0

$09 / 95$

\subsection{PROCESS DESCRIPTION}

Process descriptions for each of the four types of source water are described below.

\subsection{Surface Water Process Description} leaks.

Waste water is generated from cooling water processes and pump packing

- Cooling Water -- Noncontact cooling water is used throughout the Hanford Site for equipment such as pumps, heating, ventilating, and. air conditioning (HVAC) systems, air compressors, turbines, generators, and boiler water jackets.

- Pump Packing Leaks -- Leaks may occur around worn out or loose fitting packings. In many cases, the pump packing is made intentionally to fit loose to extend its lifetime, thus reducing the cost of frequent changes.

\subsection{Sanitary Water Process Descriptions}

Sanitary water processes contribute to the Miscellaneous Streams on the Hanford Site. The following sections describe sanitary water processes.

5.2.1 Steam Condensate. Steam is produced from sanitary water that has been dechlorinated and sent through a water softner system to remove minerals (calcium and magnesium). The treated water is introduced into coal-fired boilers to produce steam. This steam is superheated before distribution to facilities for both heating and process use. Disposal sites receive steam condensate from the steam distribution lines. When used for heating purposes, this is a seasonal discharge. Dearborn $66^{2}$, and Polyquest $683^{1}$ are added to dechlorinate the water, prevent scale, and control corrosion before entering the boilers. Super Filmeen $14^{1}$ is added at the powerhouse to the steam for corrosion control in the steam lines.

5.2.2 Cooling Water. Along with raw water, sanitary water is also used as noncontact cooling water throughout the Hanford Site for equipment such as pumps, HVAC systems, air compressors, turbines, generators, and boiler water jackets. Air compressor blowdown also is included in this category.

- HVAC -- Continuous air exchange is required in process and/or work areas. Outside air is heated or cooled as needed. Condensate is produced by the HVAC system. This condensate is the entrained moisture in the air drawn in by the respective HVAC system. This condensate is collected and discharged to the disposal site.

- Pump Packing Leaks -- Leaks may occur around worn out or loose fitting packings. In many cases, the pump packing is made

2 A trademark of Grace Dearborn Co. 
intentionally to fit loose to extend its lifetime, thus reducing the cost of frequent changes.

- Air Compressor Blowdown -- Compressed air storage tanks contain a moisture trap and drain valve. Water vapor, which condenses when the air is compressed, collects in the trap and is drained periodically. The effluent may be contaminated with small quantities of oil. The source is intermittent and the flow rate depends on compressed air demand and seasonal fluctuations in ambient air temperature and humidity.

5.2.3 Sink Drains. Sink drains collect waste water used in kitchens, cleaning processes, eye wash stations, and safety showers. The majority of sinks are used for general sanitation practices, such as washing hands, while others, like those used in paint shops, are used for cleaning painting equipment.

- Cleaning/Kitchens -- Waste water is generated from cleaning paint brushes and water jugs. Kitchens in several buildings also generate waste water.

- Eye Wash and Safety Shower -- Eye wash and safety showers are for emergency use only. These are used when a person comes in contact with hazardous materials that must be washed off their eyes and body to reduce the risk of serious injury.

\subsection{Groundwater Process Description} leaks.

Wastewater is generated from cooling water processes and pump packing

- Cooling Water -- Noncontact cooling water is used throughout the Hanford Site for equipment such as pumps, heating, ventilating, and air conditioning (HVAC) systems, air compressors, turbines, generators, and boiler water jackets.

- Pump Packing Leaks -- Leaks may occur around worn. out or loose fitting packings. In many cases, the pump packing is made intentionally to fit loose to extend its lifetime, thus reducing the cost of frequent changes.

\subsection{Storm Water Process Description}

Storm water is generated from rainfall and snowmelt that runs off of roofs, pavement, etc.. If runoff flows through areas of contamination, the runoff may aid in the transport of contaminants through the soil column. 
DOE/RL-95-82, Rev. 0

$09 / 95$

(This page intentionally left blank) 
Table 3-1. Miscellaneous Streams Inventory

\begin{tabular}{|c|c|c|c|c|c|c|c|c|c|c|c|}
\hline Area & $\begin{array}{l}\text { Source } \\
\text { Water }\end{array}$ & $\begin{array}{c}\text { Stream } \\
\#\end{array}$ & Note & Process Description & \begin{tabular}{|c|} 
Flow \\
(gpm)
\end{tabular} & Disposal Structure & $\begin{array}{l}\text { Well } \\
\text { Code }\end{array}$ & $\begin{array}{c}\text { Washington } \\
\text { State Planar } \\
\text { Coordinates } \\
\text { (meters) }\end{array}$ & Comments & 216 & 218 \\
\hline 10013 & D & 73 & & $\begin{array}{l}181 \text { B Building - cooling waler for diesel } \\
\text { emiergency pump and traveling screen } \\
\text { backwash. }\end{array}$ & $<0.50$ & Trench & $\mathrm{N} / \mathrm{A}$ & $\begin{array}{l}\text { ES64,855.48 } \\
\text { N145,220.08 }\end{array}$ & $\begin{array}{l}\text { Description revied per ce mail from } \\
\text { D. Ilerman dated 7/19/95. Travel } \\
\text { screen back wash has been out of } \\
\text { service for } 3 \text { years, sereens will be } \\
\text { replaced and placed back into } \\
\text { service. Located approximately } 60 \\
\text { feet from Columbia River }\end{array}$ & Yes & No \\
\hline 1000 & & 672 & & $\begin{array}{l}181 \mathrm{~B} \text { Building, alandoned injection well } \\
\text { located in front of building. }\end{array}$ & 0.00 & injection well & & $\begin{array}{l}\text { ES64,675.00 } \\
\text { N145,230.00 }\end{array}$ & $\begin{array}{l}\text { ELIMINATED: 7/26/95 this } \\
\text { injection well has been abandoned } \\
\text { per CC mail from D. Hlerman dated } \\
7 / 19 / 95\end{array}$ & No & No \\
\hline 10013 & D & 74 & & $\begin{array}{l}\text { 1821B Building - sanilary water from sinks, } \\
\text { drinking fountains and pump water. }\end{array}$ & $<5,00$ & Drain Field & N/A & $\begin{array}{l}\text { ES64,768.30 } \\
\text { N144,668.30 }\end{array}$ & $\begin{array}{l}\text { CORRECTED 8/94. From sump } \\
\text { collection to current description. } \\
\text { 182B Building }\end{array}$ & Yes & No \\
\hline 1001$)$ & D) & 671 & & $\begin{array}{l}\text { 181D Building. cooling water for diesel } \\
\text { emergency pumps, and traveling screen } \\
\text { backwaslı }\end{array}$ & $<0.50$ & Irench & N/A & $\mathrm{E} 0.00 \mathrm{~N} 0.00$ & $\begin{array}{l}\text { Per cc mail dated 7/19/95 from D. } \\
\text { llerman, stream was added to } \\
\text { inventory }(7 / 26 / 95) \text {. Screens have } \\
\text { not operated for } 3 \text { years but plan on } \\
\text { placing replaced sereens in service. }\end{array}$ & Yes & No \\
\hline 10010 & & 673 & & 181D Building. injection well abandoned & 0.00 & & & $\begin{array}{l}\text { E572,790.29 } \\
\text { N151,724.97 }\end{array}$ & $\begin{array}{l}\text { Eliminated: stream was added to } \\
\text { inventory per cemail from D. Ilerman } \\
\text { dated7/19/95. Injection well has } \\
\text { been abandoned. }\end{array}$ & No & No \\
\hline $100 \mathrm{k}$ & & 676 & & $\begin{array}{l}1717 \mathrm{~K} \text { Building - Evaporative cooler } \\
\text { discharge (1 Contributor) }\end{array}$ & 0.00 & & & E0.00 N0.00 & $\begin{array}{l}\text { Added 8/31/95. Stream discharges to } \\
\text { ground. }\end{array}$ & & No \\
\hline $100 k$ & & 681 & & $\begin{array}{l}1717 \mathrm{~K} \text { Building - Evaporative cooler } \\
\text { discharge (3 contributors) }\end{array}$ & 0.00 & & & E $0.00 N 0.00$ & $\begin{array}{l}\text { Added 8/31/95. Stream discharges to } \\
\text { ground. }\end{array}$ & Yes & No \\
\hline $100 \mathrm{~N}$ & $\mathrm{c}$ & 395 & & 107N Building - Rain run-off. & $<0.50$ & Injection Well & SD2 & $\begin{array}{l}\text { Es71,029.89 } \\
\text { N149,480.59 }\end{array}$ & West area of $107 \mathrm{~N}$. & Yes & Yes \\
\hline
\end{tabular}

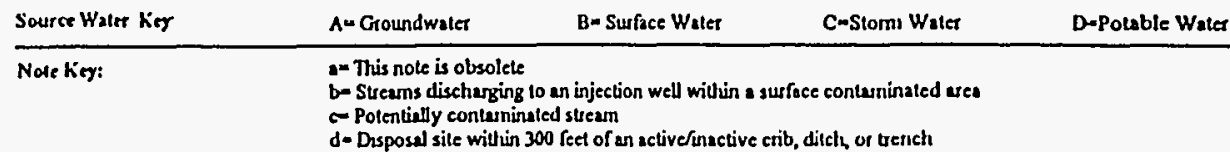


Table 3-1. Miscellaneous Streams Inventory

\begin{tabular}{|c|c|c|c|c|c|c|c|c|c|c|c|}
\hline Area & $\begin{array}{l}\text { Source } \\
\text { Water }\end{array}$ & $\begin{array}{c}\text { Stream } \\
\#\end{array}$ & Note & Process Description & $\begin{array}{c}\text { Flow } \\
\text { (gpm) }\end{array}$ & Disposal Structure & $\begin{array}{l}\text { Well } \\
\text { Code }\end{array}$ & $\begin{array}{l}\text { Washington } \\
\text { State Planar } \\
\text { Coordinates } \\
\text { (meters) }\end{array}$ & Comments & 216 & 218 \\
\hline $100 \mathrm{~N}$ & C & 396 & & 107N Building - Rain run-off. & $<0.50$ & Injection Well & SD2 & $\begin{array}{l}\text { Es71,038.12 } \\
\text { N149,477.15 }\end{array}$ & East area of $107 \mathrm{~N}$. & Yes & Yes \\
\hline $100 \mathrm{~N}$ & B & 492 & & $\begin{array}{l}\text { 183N Building - When fire system piping is } \\
\text { opened al the valve pit for repair, untreated } \\
\text { raw water from the Columbia River (via } \\
\text { Ilanford Site export water system) drains } \\
\text { from pipes into the pit. }\end{array}$ & $<0.01$ & $\begin{array}{l}\text { Injection Well at the } \\
\text { bottom of the valve pit. }\end{array}$ & SW 20 & $\begin{array}{l}\text { Es71,110.05 } \\
\text { N149,397.06 }\end{array}$ & $\begin{array}{l}\text { ADDED 11/94. Valve pit north of } \\
183 \mathrm{~N} \text {. }\end{array}$ & Yes & Yes \\
\hline $100 \mathrm{~N}$ & B & 493 & & $\begin{array}{l}183 \mathrm{~N} \text { Building - This stream is a relief valve } \\
\text { which releases during upset conditions in the } \\
\text { plant fire systents. Released waler flows into } \\
\text { a container, and overllows on the ground. }\end{array}$ & 0.00 & Discharges to the ground & & $\begin{array}{l}\text { E571,110.05 } \\
\text { N149,397.06 }\end{array}$ & $\begin{array}{l}\text { ELIMINATED: Disposal site is not } \\
\text { an engineered structurc. ADDED } \\
11 / 94 \text {. Average annual flow rate is } \\
\text { unknown. North of } 183 \mathrm{~N} \text {, adjacent } \\
\text { to Valve Pit housing the french drain } \\
\text { mentioned in } 510 \text {. }\end{array}$ & No & No \\
\hline 1100 & c & 618 & & $\begin{array}{l}1100 \text { Area parking lol storm drain system - } \\
\text { Catch Basin } \$ 32\end{array}$ & 0.00 & & & $\begin{array}{l}\text { Es93,533.22 } \\
\text { N110,439.11 }\end{array}$ & $\begin{array}{l}\text { ELIMINATED: 7/6/9S per M. } \\
\text { Gunter catch basin does not } \\
\text { discharge directly to ground. } \\
\text { ADDED 4/95. }\end{array}$ & No & No \\
\hline 1100 & C & 588 & & $\begin{array}{l}1100 \text { Area parking lot storm drain system - } \\
\text { Catch Basin } \# 1 \text {. }\end{array}$ & 0.00 & & & $\begin{array}{l}\text { E } 593,507.77 \\
\text { N } 111,066.03\end{array}$ & $\begin{array}{l}\text { ELIMINATED: } 7 / 6 / 95 \text { per } \mathrm{M} \text {. } \\
\text { Gunter these catch basins donot } \\
\text { have a direct discharge to the } \\
\text { ground. ADDED 4/95 }\end{array}$ & No & No \\
\hline 1100 & & 596 & & $\begin{array}{l}1100 \text { Area parking fol storm drain system. } \\
\text { Catch Basin } \# 10\end{array}$ & 0.00 & & & $\begin{array}{l}\text { E593,440.46 } \\
\text { N110,901.24 }\end{array}$ & $\begin{array}{l}\text { ELIMINATED. CORRECTED: } \\
7 / 6 / 95 \text { per } M \text {. Gunter: this is not an } \\
\text { injection well it is only a bend in the } \\
\text { piping. ADDED } 4 / 95 \text {. This catch } \\
\text { basin is below grade, located at a } \\
\text { bend in the piping between catch } \\
\text { basin } 19 \text { and the outlet to the } \\
\text { collection system. }\end{array}$ & No & No \\
\hline 1100 & c & 597 & & $\begin{array}{l}1100 \text { Area parking lot storm drain system. } \\
\text { Calch Basin } \# 11\end{array}$ & 0.00 & & & $\begin{array}{l}\text { ES93,618.17 } \\
\text { N110,721.23 }\end{array}$ & $\begin{array}{l}\text { ELIMINATED: 7/6/95 per M. } \\
\text { Gunter catch basin does nol directly } \\
\text { discharge to ground. ADDED 4/95. }\end{array}$ & No & No \\
\hline
\end{tabular}

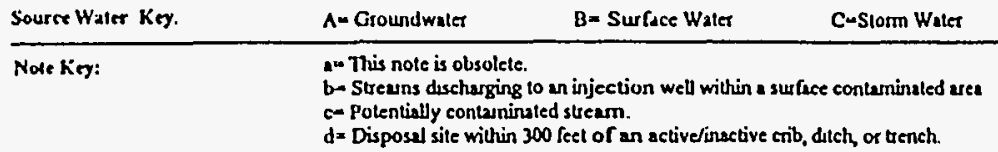

D.Polable Waler 
Table 3-1. Miscellaneous Streams Inventory

\begin{tabular}{|c|c|c|c|c|c|c|c|c|c|c|c|}
\hline Area & $\begin{array}{l}\text { Source } \\
\text { Water }\end{array}$ & $\begin{array}{c}\text { Stream } \\
\#\end{array}$ & Note & Process Description & $\begin{array}{c}\text { Flow } \\
\text { (gpm) }\end{array}$ & Disposal Structure & \begin{tabular}{l|} 
Well \\
Code
\end{tabular} & $\begin{array}{c}\text { Washington } \\
\text { State Planar } \\
\text { Coordinates } \\
\text { (meters) } \\
\end{array}$ & Comments & 216 & 218 \\
\hline 1100 & c & 598 & & $\begin{array}{l}1100 \text { Area parking lot storm drain system - } \\
\text { Catch Basin } \# 12\end{array}$ & 0.00 & & & $\begin{array}{l}\text { ES93.601.25 } \\
\text { N110.721.23 }\end{array}$ & $\begin{array}{l}\text { ELIMINATED: } 7 / 6 / 95 \text { per M. } \\
\text { Gunter calch basin does not } \\
\text { discharge directly to ground. } \\
\text { ADDED 4/95. }\end{array}$ & No & No \\
\hline 1100 & c & 599 & & $\begin{array}{l}1100 \text { Arca parking lol storm drain system - } \\
\text { Catcl Basin } \# 13\end{array}$ & 0.00 & & & $\begin{array}{l}\text { ES93,548.67 } \\
\text { N110,721.23 }\end{array}$ & $\begin{array}{l}\text { ELIMINATED: } 7 / 6 / 95 \text { per } M . \\
\text { Gunter calch basin does nol } \\
\text { discharge directly to ground. } \\
\text { ADDED } 4 / 95\end{array}$ & No & No \\
\hline 1100 & C & 600 & & $\begin{array}{l}1100 \text { Area parking lot storm drain system - } \\
\text { Catch Basin } \| 14\end{array}$ & 0.00 & & & $\begin{array}{l}\text { ES93,618.17 } \\
\text { N110,666.76 }\end{array}$ & $\begin{array}{l}\text { ELIMINATED: } 7 / 6 / 95 \text { per M. } \\
\text { Gunter catch basin does not } \\
\text { discharge directly to ground. } \\
\text { ADDED 4/95 }\end{array}$ & No & No \\
\hline 1100 & C & 601 & & $\begin{array}{l}1100 \text { Area parking lol storm drain system - } \\
\text { Catch Basin } \# 15\end{array}$ & 0.00 & & & $\begin{array}{l}\text { ES93,602.10 } \\
\text { N110,666.76 }\end{array}$ & $\begin{array}{l}\text { ELIMINATED: 7/6/95 per M. } \\
\text { Gunter catch basin does not } \\
\text { discharge directly to ground. } \\
\text { ADDED 4/95 }\end{array}$ & No & No \\
\hline 1100 & $\mathrm{c}$ & 602 & & $\begin{array}{l}1100 \text { Area parking lot storm drain system.- } \\
\text { Catch Basin } \# 16\end{array}$ & 0.00 & & & $\begin{array}{l}\text { ES93,602.10 } \\
\text { N110,666.76 }\end{array}$ & $\begin{array}{l}\text { ELIMINATED: 7/6/95 per M. } \\
\text { Gunter catch basin does not } \\
\text { discharge directly to ground. } \\
\text { ADDED 4/95. }\end{array}$ & No & No \\
\hline 1100 & c & 603 & & $\begin{array}{l}1100 \text { Area parking lot storm drain system - } \\
\text { Catch Basin } \# 17\end{array}$ & 0.00 & & & $\begin{array}{l}\text { E593,566.96 } \\
\text { N110,698.55 }\end{array}$ & $\begin{array}{l}\text { ELIMINATED: 7/6/95 per M. } \\
\text { Gunter calch basin does not } \\
\text { discharge directly to ground. } \\
\text { ADDED 4/95. }\end{array}$ & No & No \\
\hline 1100 & c & 604 & & $\begin{array}{l}1100 \text { Area parking lot stom drain system - } \\
\text { Catch Basin } / 18\end{array}$ & $<0.00$ & & & $\begin{array}{l}\text { ES93,544.04 } \\
\text { N110,698.53 }\end{array}$ & $\begin{array}{l}\text { ELIMINATED: 7/6/95 per M. } \\
\text { Gunter calch basin does not } \\
\text { discharge directly to ground. } \\
\text { ADDED 4/95. }\end{array}$ & No & $\overline{\text { No }}$ \\
\hline 1100 & c & 605 & & $\begin{array}{l}1100 \text { Area parking lot stom drain system - } \\
\text { Catch Basin } \# 19\end{array}$ & 0.00 & & & $\begin{array}{l}\text { E593,533.22 } \\
\text { N110,702.45 }\end{array}$ & $\begin{array}{l}\text { ELIMINATED: 7/6/95 per M. } \\
\text { Gunter, catch basin does not } \\
\text { disclarge direclly to ground. } \\
\text { ADDEED 4/95. }\end{array}$ & No & No \\
\hline
\end{tabular}


Table 3-1. Miscellaneous Streams Inventory

\begin{tabular}{|c|c|c|c|c|c|c|c|c|c|c|c|}
\hline Area & $\begin{array}{l}\text { Source } \\
\text { Water }\end{array}$ & $\begin{array}{c}\text { Stream } \\
\#\end{array}$ & Note & Process Description & $\begin{array}{c}\text { Flow } \\
\text { (gpm) }\end{array}$ & Disposal Structure & $\begin{array}{l}\text { Well } \\
\text { Code }\end{array}$ & $\begin{array}{c}\text { Washington } \\
\text { State Planar } \\
\text { Coordinates } \\
\text { (meters) } \\
\end{array}$ & Comments & 216 & 218 \\
\hline 1100 & $c$ & 589 & & $\begin{array}{l}1100 \text { Area parking lot storm drain systetn - } \\
\text { Calch Basin / } 2\end{array}$ & 0.00 & & & $\begin{array}{l}\text { Es93,509.30 } \\
N 110,975.13\end{array}$ & $\begin{array}{l}\text { ELIMINATED: } 7 / 6 / 95 \text { per } M \text {. } \\
\text { Gunter the catch basin does not } \\
\text { discharge directly to ground. } \\
\text { ADDED 4/95. }\end{array}$ & No & No \\
\hline 1100 & $c$ & 606 & & $\begin{array}{l}1100 \text { Area parking lot storm drain system - } \\
\text { Catch Basin // } 20\end{array}$ & 0.00 & & & $\begin{array}{l}\text { E593,533.22 } \\
\text { N110,698.56 }\end{array}$ & $\begin{array}{l}\text { ELIMINATED: 7/6/95 per M. } \\
\text { Gunter, catch basin does not } \\
\text { discharge directly to ground. } \\
\text { ADDED 4/95 }\end{array}$ & No & No \\
\hline 1100 & c & 607 & & $\begin{array}{l}1100 \text { Area parking lot storm drain system - } \\
\text { Catch 13:sin } 121\end{array}$ & 0.00 & & & $\begin{array}{l}\text { E593,469.57 } \\
N 110,698.56\end{array}$ & $\begin{array}{l}\text { ELIMINATED: } 7 / 6 / 95 \text { per M. } \\
\text { Gunter catch basin does nol } \\
\text { discharge directly to ground. } \\
\text { ADDED 4/95 }\end{array}$ & No & No \\
\hline 1100 & $\mathrm{c}$ & 608 & & $\begin{array}{l}1100 \text { Area parking lot storm drain system - } \\
\text { Calch Basin } 1 / 22\end{array}$ & 0.00 & & & $\begin{array}{l}\text { ES93,587.32 } \\
\text { N110,566.88 }\end{array}$ & $\begin{array}{l}\text { ELIMINATED: 7/6/95 per M. } \\
\text { Gunter catch basin does not } \\
\text { discharge directly to ground. } \\
\text { ADDED 4/95. }\end{array}$ & No & No \\
\hline 1100 & $\mathrm{c}$ & 609 & & $\begin{array}{l}1100 \text { Area parking lot storm drain system - } \\
\text { Catch Basin \# } 23\end{array}$ & 0.00 & & & $\begin{array}{l}\text { E593,574.06 } \\
\text { N110,566.88 }\end{array}$ & $\begin{array}{l}\text { ELIMINATED: 7/6/95 per M. } \\
\text { Gunter catch basin does not } \\
\text { discharge directly to ground. } \\
\text { ADDED 4/95. }\end{array}$ & No & No \\
\hline 1100 & c & 610 & & $\begin{array}{l}1100 \text { Area parking lot stonn drain syslem - } \\
\text { Catch Basin } / 24\end{array}$ & 0.00 & & & $\begin{array}{l}\text { E.S93,544.04 } \\
\text { N110,566.88 }\end{array}$ & $\begin{array}{l}\text { ELIMINATED: 7/6/95 per M. } \\
\text { Gunter catch basin does not } \\
\text { discharge directly to ground. } \\
\text { ADDED 4/95. }\end{array}$ & No & No \\
\hline 1100 & c & 611 & & $\begin{array}{l}1100 \text { Area parking lot storm drain system - } \\
\text { Calch Basin } 1225\end{array}$ & 0.00 & & & $\begin{array}{l}\text { ES593,533.22 } \\
\text { N110,570.51 }\end{array}$ & $\begin{array}{l}\text { ELIMINATED: } 7 / 6 / 95 \text { per M. } \\
\text { Gunter calch basin does not } \\
\text { dischlarge directly to ground. } \\
\text { ADDED 4/95. }\end{array}$ & No & No \\
\hline 1100 & $c$ & 612 & & $\begin{array}{l}1100 \text { Area parking lot storn drain system - } \\
\text { Catch Basin } \# 26\end{array}$ & 0.00 & & & $\begin{array}{l}\text { ES93,533.22 } \\
\text { N110,566.88 }\end{array}$ & $\begin{array}{l}\text { FLIMINATED: 7/6/95 per M. } \\
\text { Gunter catch basin does not } \\
\text { discharge directly to ground. } \\
\text { ADDED 4/95. }\end{array}$ & No & No \\
\hline \multicolumn{12}{|c|}{ Sourre Water Keg } \\
\hline
\end{tabular}


Table 3-1. Miscellaneous Streams Inventory

\begin{tabular}{|c|c|c|c|c|c|c|c|c|c|c|c|}
\hline Area & $\begin{array}{l}\text { Source } \\
\text { Water }\end{array}$ & $\begin{array}{c}\text { Stream } \\
\#\end{array}$ & Note & Process Description & $\begin{array}{l}\text { Flow } \\
\text { (gpm) }\end{array}$ & Disposal Structure & $\begin{array}{l}\text { Well } \\
\text { Code }\end{array}$ & $\begin{array}{c}\text { Washington } \\
\text { State Planar } \\
\text { Coordinates } \\
\text { (meters) } \\
\end{array}$ & Comments & 216 & 218 \\
\hline 1100 & c & 613 & & $\begin{array}{l}1100 \text { Area parking lot slomu drain system - } \\
\text { Catch Basin } \| 27\end{array}$ & 0.00 & & & $\begin{array}{l}\text { E593,469.57 } \\
\text { N110,566.88 }\end{array}$ & $\begin{array}{l}\text { ELIMINATED: 7/6/95 per M. } \\
\text { Gunter calch basin does not } \\
\text { discharge directly to ground. } \\
\text { ADDED 4/95. }\end{array}$ & No & No \\
\hline 1100 & c & 615 & . & $\begin{array}{l}1100 \text { Area parking lot storm drain system - } \\
\text { Catch Basin } 1129\end{array}$ & 0.00 & & & $\begin{array}{l}\text { ES93,585.03 } \\
\text { N110,433.44 }\end{array}$ & $\begin{array}{l}\text { ELIMINATED: 7/6/95 per M. } \\
\text { Ounter catch basin does not } \\
\text { discharge directly to ground. } \\
\text { ADDED 4/95. }\end{array}$ & No & No \\
\hline 1100 & c & 590 & & $\begin{array}{l}1100 \text { Area parking lot slorm drain system - } \\
\text { Calcl Basin } \| 3\end{array}$ & 0.00 & & & $\begin{array}{l}\text { ES93,510.41 } \\
\text { N110,907.16 }\end{array}$ & $\begin{array}{l}\text { ELIMINATED: 7/6/95 per M. } \\
\text { Ounter, calch basin does not } \\
\text { discharge directly to ground. } \\
\text { ADDED 4/95. }\end{array}$ & No & No \\
\hline 1100 & c & 616 & & $\begin{array}{l}1100 \text { Area parking lot storm drain system - } \\
\text { Calch Basin \# } 30\end{array}$ & 0.00 & & & $\begin{array}{l}\text { ES93,585.03 } \\
\mathrm{N} 110,436.27\end{array}$ & $\begin{array}{l}\text { ELIMINATED: 7/6/95 per M. } \\
\text { Gunter catch basin does not } \\
\text { discharge directly to ground. } \\
\text { ADDED 4/95. }\end{array}$ & No & No \\
\hline 1100 & c & 617 & & $\begin{array}{l}1100 \text { Area parking lot storm drain system - } \\
\text { Calch Basin \# } 31\end{array}$ & 0.00 & & & $\begin{array}{l}\text { E593,544.04 } \\
\text { N110,436.28 }\end{array}$ & $\begin{array}{l}\text { ELIMINATED: 7/6/95 per M. } \\
\text { Gunter catch basin does not directly } \\
\text { to ground. ADDED 4/95. }\end{array}$ & No & No \\
\hline 1100 & & 619 & & $\begin{array}{l}1100 \text { Area parking lot stom drain system - } \\
\text { Calch Basin } \# 33\end{array}$ & 0.00 & & & $\begin{array}{l}\text { ES S93,533.22 } \\
\text { N110,436.03 }\end{array}$ & $\begin{array}{l}\text { ELIMINATED: CORRECTED: } \\
\text { 7/6/95 per M. Gunter: is only a } \\
\text { bend in the pipe, not a disposal site. } \\
\text { ADDED 4/95. This catch basin is } \\
\text { below grade, located al a wye in the } \\
\text { piping adjacent to calch basin } 1 / 32 \text {. }\end{array}$ & No & No \\
\hline 1100 & c & 620 & & $\begin{array}{l}1100 \text { Arca parking lot storm drain system - } \\
\text { Calch Basin } 134\end{array}$ & 0.00 & & & $\begin{array}{l}\text { ES93,491.45 } \\
\text { N } 110,435.12\end{array}$ & $\begin{array}{l}\text { ELIMINATID: 7/6/95 per M. } \\
\text { Gunter catch basin does not } \\
\text { discharge directly to ground. } \\
\text { ADDED 4/95. }\end{array}$ & No & No \\
\hline
\end{tabular}

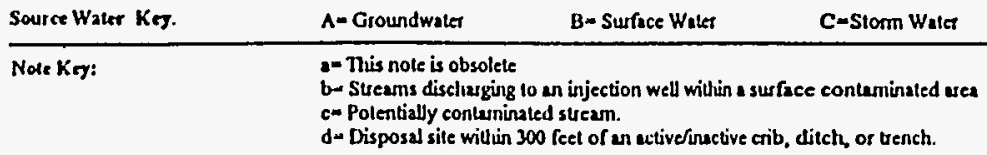


Table 3-1. Miscellaneous Streams Inventory

\begin{tabular}{|c|c|c|c|c|c|c|c|c|c|c|c|}
\hline Area & \begin{tabular}{|c|}
$\begin{array}{c}\text { Source } \\
\text { Water }\end{array}$ \\
\end{tabular} & $\begin{array}{c}\text { Stream } \\
\#\end{array}$ & Note & Process Description & $\begin{array}{c}\text { Flow } \\
\text { (gpm) }\end{array}$ & Disposal Structure & \begin{tabular}{|l|} 
Well \\
Code
\end{tabular} & $\begin{array}{c}\text { Washington } \\
\text { State Planar } \\
\text { Coordinates } \\
\text { (meters) }\end{array}$ & Comments & 216 & 218 \\
\hline 1100 & $\mathrm{c}$ & 621 & & $\begin{array}{l}1100 \text { Area parking lot storm drain syslem - } \\
\text { Catch Basin \# } 35\end{array}$ & 0.00 & & & $\begin{array}{l}\text { E } 593,481.24 \\
\text { N } 110,445.97\end{array}$ & $\begin{array}{l}\text { ELIMINATED: 7/6/95 per M. } \\
\text { Gunter catch basin does not } \\
\text { discharge directly to ground. } \\
\text { ADDED 4/95. }\end{array}$ & No & No \\
\hline 1100 & $\mathrm{c}$ & 622 & & $\begin{array}{l}1100 \text { Area parking lot storm drain system - } \\
\text { Catch Basin } \# 36\end{array}$ & 0.00 & & & $\begin{array}{l}\text { E } 593,440.30 \\
\text { N } 110,489.40\end{array}$ & $\begin{array}{l}\text { ELIMINATED:: 7/6/95 per M. } \\
\text { Gunter catch basin does not } \\
\text { discharge dirzcelty to ground.. } \\
\text { ADDED 4/95. This catch basin is } \\
\text { located below grade, located at a } 45 \\
\text { degree bend in the piping between } \\
\text { catch basin t36 and the collection } \\
\text { system oullet point. }\end{array}$ & No & No \\
\hline 1100 & c & 591 & & $\begin{array}{l}1100 \text { Area parking lot storm drain system - } \\
\text { Calch Basin } \$ 5\end{array}$ & 0.00 & & & $\begin{array}{l}E 593,611.31 \\
\text { N110,833.85 }\end{array}$ & $\begin{array}{l}\text { ELIMINATED: } 7 / 6 / 95 \text { per M. } \\
\text { Gunter catch basin does not } \\
\text { discharge directly to ground. } \\
\text { ADDED 4/95 }\end{array}$ & No & No \\
\hline 1100 & c & 592 & & $\begin{array}{l}1100 \text { Area parking lot storm drain system - } \\
\text { Catch Basin } \# 6\end{array}$ & 0.00 & & & $\begin{array}{l}\text { ES93,553.64 } \\
\text { N110,833.98 }\end{array}$ & $\begin{array}{l}\text { ELIMINATED: 7/6/95 per M. } \\
\text { Gunter catch basin does not } \\
\text { discharge directly to ground. } \\
\text { ADDED 4/95. }\end{array}$ & No & No \\
\hline 1100 & c & 593 & & $\begin{array}{l}1100 \text { Area parking lot storn drain system - } \\
\text { Catch Basin } \# 7 \text {. }\end{array}$ & 0.00 & & & $\begin{array}{l}\text { ES93,510.48 } \\
\text { N110,833.98 }\end{array}$ & $\begin{array}{l}\text { ELIMINATED: } 7 / 6 / 95 \text { per M. } \\
\text { Gunter catch basin does not } \\
\text { discharge directly to ground. } \\
\text { ADDED } 4 / 95 \text {. }\end{array}$ & No & No \\
\hline 1100 & $\mathrm{c}$ & 594 & & $\begin{array}{l}1100 \text { Area parking lot storm drain system - } \\
\text { Catch Basin } \# 8 \text {. }\end{array}$ & 0.00 & & & $\begin{array}{l}\text { ES93,469.57 } \\
\text { N110,833.98 }\end{array}$ & $\begin{array}{l}\text { ELIMINATED: } 7 / 6 / 95 \text { per M. } \\
\text { Gunter, catch basin does not } \\
\text { discharge directly to ground. } \\
\text { ADDED 4/95. }\end{array}$ & No & No \\
\hline 1100 & c & 595 & & $\begin{array}{l}1100 \text { Area parking lot storm drain system - } \\
\text { Catch Basin } \# 9\end{array}$ & 0.00 & & & $\begin{array}{l}\text { ES93,435.59 } \\
N 110,006.12\end{array}$ & $\begin{array}{l}\text { ELIMINATED: } 7 / 6 / 95 \text { per M. } \\
\text { Gunter catch basin does not } \\
\text { discliarge directly to ground. } \\
\text { ADDI:D 4/95. }\end{array}$ & No & No \\
\hline uree $w$ & rater Keg. & $A=$ & Groundwatc & B- Surface Water & & D-Pouble Water & & & & & \\
\hline
\end{tabular}


Table 3-1. Miscellaneous Streams Inventory

\begin{tabular}{|c|c|c|c|c|c|c|c|c|c|c|c|}
\hline Area & $\begin{array}{l}\text { Source } \\
\text { Water }\end{array}$ & $\begin{array}{c}\text { Stream } \\
\#\end{array}$ & Note & Process Description & $\begin{array}{c}\text { Flow } \\
\text { (gpm) }\end{array}$ & Disposal Structure & $\begin{array}{l}\text { Well } \\
\text { Code }\end{array}$ & $\begin{array}{l}\text { Washington } \\
\text { State Planar } \\
\text { Coordinates } \\
\text { (meters) }\end{array}$ & Comments & 216 & 218 \\
\hline 1100 & c & 614 & & $\begin{array}{l}1100 \text { Area parking lot stonn drain system - } \\
\text { Catch Basin } \# 28\end{array}$ & 0.00 & & & $\begin{array}{l}\text { Es93,585.03 } \\
\text { N110,411.10 }\end{array}$ & $\begin{array}{l}\text { ELIMINATED: 7/6/95 per M. } \\
\text { Gunter calch basin does not } \\
\text { discharge directly to ground. } \\
\text { ADDED 4/95. }\end{array}$ & No & No \\
\hline 1100 & c & 661 & & $\begin{array}{l}1163 \text { Building - Stom water run-olr from } \\
\text { parking area flows to catcl basin; overflow } \\
\text { routed to a drywell beneath catch basin. } \\
\end{array}$ & $<0.01$ & injection well & SD2 & $\begin{array}{l}\text { E591,660.00 } \\
\text { N123,654.00 }\end{array}$ & $\begin{array}{l}\text { ADDED: 7/6/95 per fax from } M \text {. } \\
\text { Gunter daled 7/6/95 }\end{array}$ & Yes & Yes \\
\hline 1100 & C & 475 & & $\begin{array}{l}1163 \text { Building - Parking Arca - Storm water } \\
\text { run-off from parking area lows to catch } \\
\text { basin; overflow routed to a drywell beneath } \\
\text { catch basin. }\end{array}$ & $<0.01$ & Injection Well & SD2 & $\begin{array}{l}\text { E592,146.71 } \\
\text { N123,939.32 }\end{array}$ & $\begin{array}{l}\text { ADDED 10/94. Parking area east of } \\
1163 \text { Building }\end{array}$ & Yes & Yes \\
\hline 1100 & C & 476 & & $\begin{array}{l}1163 \text { Building - Parking Area - Storm waler } \\
\text { run-off from parking area flows to catch } \\
\text { basin; overflow routed to a drywell beneath } \\
\text { catch basin. }\end{array}$ & $<0.01$ & Injection Well & 5D2 & $\begin{array}{l}\text { E592,152.39 } \\
\text { N123,859.18 }\end{array}$ & $\begin{array}{l}\text { ADDED 10/94. Parking Area east } \\
\text { of } 1163 \text { Building }\end{array}$ & Yes & Yes \\
\hline 1100 & $\mathrm{c}$ & 477 & & $\begin{array}{l}1163 \text { Duilding - Parking area - Storm water } \\
\text { run-off from parking area flows to catch } \\
\text { basin; overllow routed to a drywell beneath } \\
\text { catch basin. }\end{array}$ & $<0.01$ & Injection Well & SD2 & $\begin{array}{l}\text { E592,147.08 } \\
\text { N123,789.67 }\end{array}$ & $\begin{array}{l}\text { ADDED 10/94. Parking area east of } \\
1163 \text { building }\end{array}$ & Yes & Yes \\
\hline 1100 & $\mathbf{c}$ & 623 & & $\begin{array}{l}1163 \text { Building - South parking lot.catch } \\
\text { basin outfall. This structure discharges } \\
\text { stormwater nunoff from } 9 \text { catch basins } \\
\text { throughout the parking lot to a ditch along } \\
\text { Snyder road. }\end{array}$ & $<0.09$ & $\begin{array}{l}\text { Outfall (discharges to a } \\
\text { ditch) }\end{array}$ & N/A & $\begin{array}{l}\text { E592,147.08 } \\
\text { N123,789.67 }\end{array}$ & $\begin{array}{l}\text { ADDED 4/95. Located adjacent to } \\
\text { Snyder road, west of the westemmost } \\
\text { driveway. }\end{array}$ & Yes & No \\
\hline 1100 & c & 669 & & $\begin{array}{l}1163 \text { Building - Stomm water nun-off from } \\
\text { parking area flows to catch basin; overflow } \\
\text { rouled to a drywell benealh catch basin. }\end{array}$ & $<0.01$ & injection well & SD2 & $\begin{array}{l}\text { E } 591,933.00 \\
N 123,554.00\end{array}$ & $\begin{array}{l}\text { ADDED: 7/6/95 per fax from } M \text {. } \\
\text { Gunter }\end{array}$ & Yes & Yes \\
\hline 1100 & c & 658 & & $\begin{array}{l}1163 \text { Building - Storm water run-off from } \\
\text { parking area llows to calch basin; overflow } \\
\text { routed to a drywell beneath catcli basin. }\end{array}$ & $<0.01$ & injection well & SD2 & $\begin{array}{l}\text { E591,933.00 } \\
\text { N123,454.00 }\end{array}$ & $\begin{array}{l}\text { ADDED: } 7 / 6 / 95 \text { per cc:mail from } M \text {. } \\
\text { Gunter dated } 6 / 30 / 95 \text {. }\end{array}$ & Yes & Yes \\
\hline 1300 & c & 474 & & $\begin{array}{l}1163 \text { Building - Storn water nun-ofr from } \\
\text { parking area flows to catch basin; overflow } \\
\text { routed to a drywell beneath catch basin. }\end{array}$ & $<0.01$ & Injection Well & SD2 & $\begin{array}{l}\text { E.592,164.06 } \\
\text { N123,947.29 }\end{array}$ & $\begin{array}{l}\text { ADDED 10/94. Parking arca cast of } \\
\text { 1 } 163 \text { Building }\end{array}$ & Yes & Yes \\
\hline
\end{tabular}

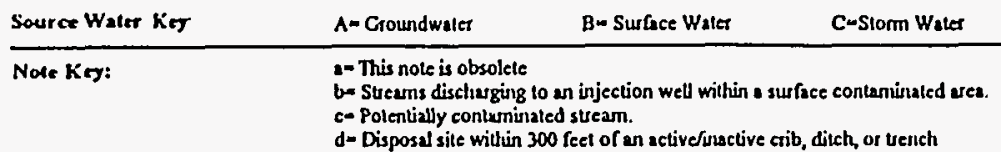

D-Potable Waler 
Table 3-1. Miscellaneous Streams Inventory

\begin{tabular}{|c|c|c|c|c|c|c|c|c|c|c|c|}
\hline Area & $\begin{array}{l}\text { Source } \\
\text { Water }\end{array}$ & $\begin{array}{c}\text { Stream } \\
\#\end{array}$ & Note & Process Description & $\begin{array}{l}\text { Flow } \\
\text { (gpm) }\end{array}$ & Disposal Structure & $\begin{array}{l}\text { Well } \\
\text { Code }\end{array}$ & $\begin{array}{l}\text { Washington } \\
\text { State Planar } \\
\text { Coordinates } \\
\text { (meters) }\end{array}$ & Comments & 216 & 218 \\
\hline 1100 & C & 478 & & $\begin{array}{l}1163 \text { Duilding - Storm water run-off from } \\
\text { parking area flows to catch basin; overflow } \\
\text { routed to a drywell beneath calch basin. }\end{array}$ & $<0.01$ & Injection Well & SD2 & $\begin{array}{l}E 592,070.75 \\
N 123,968.09\end{array}$ & $\begin{array}{l}\text { ADDED 10/94. North of } 1163 \\
\text { Building }\end{array}$ & Yes & Yes \\
\hline 1100 & c & 662 & & $\begin{array}{l}1163 \text { Building - Stom water nun-off from } \\
\text { parking area flows to catch basin; overilow } \\
\text { routed to a drywell beneath catch basin. }\end{array}$ & $<0.01$ & injection well & SD2 & $\begin{array}{l}\text { E592,361.00 } \\
N 123,994.00\end{array}$ & $\begin{array}{l}\text { ADDED: 7/6/95 per fax from } M \text {. } \\
\text { Ounter. }\end{array}$ & Yes & Yes \\
\hline 1100 & $\mathrm{C}$ & 663 & & \begin{tabular}{|l|}
163 Building - Storm water run-off from \\
parking area flows to catch basin; overflow \\
routed to a drywell benealh catch basin. \\
\end{tabular} & $<0.01$ & injection wells & SD2 & $\begin{array}{l}E 592,199.00 \\
\text { N123,791.00 }\end{array}$ & $\begin{array}{l}\text { ADDED: } 7 / 6 / 95 \text { per fax from } M \text {. } \\
\text { Gunter }\end{array}$ & Yes & Yes \\
\hline 1100 & $\mathrm{c}$ & 664 & & $\begin{array}{l}1163 \text { Building - Storm water run-ofr from } \\
\text { parking area flows to catch basin; overflow } \\
\text { routed to a drywell beneath catch basin. }\end{array}$ & $<0.01$ & injection well & SD2 & $\begin{array}{l}\text { E592,033.00 } \\
\text { N123,651.00 }\end{array}$ & $\begin{array}{l}\text { ADDED: 7/6/95 per fax from M. } \\
\text { Gunter }\end{array}$ & Yes & Yes \\
\hline 1100 & C & 665 & & $\begin{array}{l}1163 \text { Building - Storm water run-off from } \\
\text { parking area flows to catch basin; overflow } \\
\text { routed to a drywell beneath catch basin. }\end{array}$ & $<0.01$ & injection well & SD2 & $\begin{array}{l}\text { ES92,033.00 } \\
\text { N123,554.00 }\end{array}$ & $\begin{array}{l}\text { ADDED: 7/6/95 per fax from } M \text {. } \\
\text { Gunter }\end{array}$ & Yes & $\overline{\text { Yes }}$ \\
\hline 1100 & C & 666 & & $\begin{array}{l}1163 \text { Building - Stom water run-off from } \\
\text { parking area flows to catch basin; overflow } \\
\text { routed to a drywell beneath catch basin. }\end{array}$ & $<0.01$ & injection well & SD2 & $\begin{array}{l}E S 92,033.00 \\
N 123,454.00\end{array}$ & $\begin{array}{l}\text { ADDED: 7/6/95 per fax from } M \text {. } \\
\text { Gunler }\end{array}$ & Yes & $\overline{\text { Yes }}$ \\
\hline 1100 & $\mathrm{c}$ & 667 & & $\begin{array}{l}1163 \text { Building - Storm water run-off from } \\
\text { parking area flows to catch basin; overflow } \\
\text { rouled to a drywell beneath catch basin. }\end{array}$ & $<0.01$ & injection well & SD2 & $\begin{array}{l}\text { ES91,983.00 } \\
\text { N123,554.00 }\end{array}$ & $\begin{array}{l}\text { ADDED: 7/6/95 per fax from M. } \\
\text { Gunter }\end{array}$ & Yes & Yes \\
\hline 1100 & $\bar{c}$ & 668 & & $\begin{array}{l}1163 \text { Building - Storm water run-off from } \\
\text { parking area llows to calch basin; overflow } \\
\text { routed to a drywell beneath catch basin. }\end{array}$ & $<0.01$ & injection well & SD2 & $\begin{array}{l}\text { E591,983.00 } \\
\text { N123,454.00 }\end{array}$ & $\begin{array}{l}\text { ADDED: 7/6/95 per fax from M. } \\
\text { Gunler }\end{array}$ & Yes & Yes \\
\hline 1100 & $\mathrm{C}$ & 539 & & 1163 Building parking lot - Stonn water. & $<1.00$ & Injection Well & SD2 & $\begin{array}{l}\text { ES43,460.00 } \\
\text { N1 } 10,200.00\end{array}$ & $\begin{array}{l}\text { ADDED 2/95. Not yet active. Should } \\
\text { be installed } 2 / 95 \text {. North parking lot } \\
\text { of } 1163 \text {. }\end{array}$ & Yes & Yes \\
\hline 1100 & c & 487 & & $\begin{array}{l}1171 \text { Building - Stonnwater Collection } \\
\text { System. Collects stonnwater overflow from } \\
\text { parking area catch basins; eflluent is used to } \\
\text { water the grass within the collection basin. }\end{array}$ & 5.00 & Collection Basin & N/A & $\begin{array}{l}\text { E592,014.55 } \\
\text { N124,516.27 }\end{array}$ & $\begin{array}{l}\text { ADDED 10/94. South of Building } \\
1171\end{array}$ & Yes & No \\
\hline
\end{tabular}

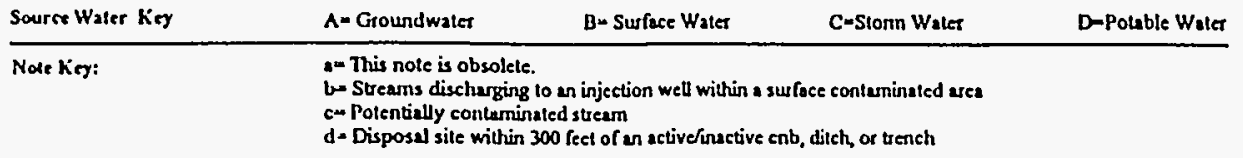


Table 3-1. Miscellaneous Streams Inventory

\begin{tabular}{|c|c|c|c|c|c|c|c|c|c|c|c|}
\hline Area & $\begin{array}{l}\text { Source } \\
\text { Water }\end{array}$ & $\begin{array}{c}\text { Stream } \\
\#\end{array}$ & Note & Process Description & $\begin{array}{c}\text { Flow } \\
\text { (gpm) }\end{array}$ & Disposal Structure & \begin{tabular}{l|} 
Well \\
Code
\end{tabular} & $\begin{array}{c}\text { Washington } \\
\text { State Planar } \\
\text { Coordinates } \\
\text { (meters) }\end{array}$ & Comments & 216 & 218 \\
\hline 1100 & $\mathrm{CD}$ & 473 & & $\begin{array}{l}1171 \text { Building - Stom water rutn-off and } \\
\text { velicle wash efluent to catch basin. }\end{array}$ & $<0.01$ & Injection Well & SD2. & $\begin{array}{l}\text { Es92,009.42 } \\
\text { N124,872.56 }\end{array}$ & $\begin{array}{l}\text { ELIMINATED: } 67 / 6 / 95 \text { per cc:mail } \\
\text { from M. Gunter dated } 6 / 30 / 95 \text {. } \\
\text { ADDED 10/94. North of } 1171 \\
\text { Building }\end{array}$ & Yes & Yes \\
\hline 1100 & $\mathbf{C}$ & 472 & & 1171 Building - parking lot stom drain. & $<0.01$ & Injection Well & SD2 & $\begin{array}{l}E 592,085.30 \\
\text { N124,928.92 }\end{array}$ & $\begin{array}{l}\text { ELIMINATED 4/95. Removed } \\
\text { during re-surfacing. ADDED 10/94. } \\
\text { Northeast of } 1171 \text { Building. To be } \\
\text { removed as paving continues. }\end{array}$ & No & No \\
\hline 1100 & c & 479 & & $\begin{array}{l}\text { Parking area in front of gravel area that was } \\
\text { once } 1166 \text { Building - Storm water run-orf. }\end{array}$ & $<0.50$ & Injection Well & 5D2 & $\begin{array}{l}\text { ES22,164.06 } \\
\text { N124,073.17 }\end{array}$ & $\begin{array}{l}\text { ADDED 10/94. Parallell to Stevens } \\
\text { Drive, between N1500 and N2200, } \\
\text { in the paved area nearest the street }\end{array}$ & Yes & Yes \\
\hline 1100 & C & 480 & & $\begin{array}{l}\text { P'arking area in front of gravel area that was } \\
\text { once } 1166 \text { Building - Stom water nun-off. }\end{array}$ & $<0.50$ & Injection Well & SD2 & $\begin{array}{l}\text { E592,164.02 } \\
\text { N124,089.93 }\end{array}$ & $\begin{array}{l}\text { ADDED 10/94. Parallell to Stevens } \\
\text { Drive, between N1 } 500 \text { and N2200, } \\
\text { in the paved area nearest the street }\end{array}$ & Yes & $\overline{\text { Yes }}$ \\
\hline 1100 & C & 481 & & $\begin{array}{l}\text { P'arking area in front of gravel area that was } \\
\text { once } 1166 \text { Building - Storm water nun-orf. }\end{array}$ & $<0.50$ & Injection Well & 5D2 & $\begin{array}{l}\text { Es92,163.99 } \\
\text { N124,103.65 }\end{array}$ & $\begin{array}{l}\text { ADDED 10/94. Parallell to Stevens } \\
\text { Drive, between N1500 and N2200, } \\
\text { in the paved area nearest the street }\end{array}$ & Yes & Yes \\
\hline 1100 & C & 482 & & $\begin{array}{l}\text { Parking area in front of gravel area that was } \\
\text { once } 1166 \text { Building - Storm water nun-off. }\end{array}$ & $<0.50$ & Injection Well & SD2 & $\begin{array}{l}\text { Es92,163.95 } \\
\text { N } 124,118.88\end{array}$ & $\begin{array}{l}\text { ADDED 10/94. Parallell to Stevens } \\
\text { Drive, between N1500 and N2200, } \\
\text { in the paved area nearest the street }\end{array}$ & Yes & Yes \\
\hline 1100 & C & 483 & & $\begin{array}{l}\text { Parking area in front of gravel area that was } \\
\text { once } 1166 \text { Building - Slorm water nun-off. }\end{array}$ & $<0.50$ & Injection Well & SD2 & $\begin{array}{l}\text { ES92,163.91 } \\
\text { N124,135.65 }\end{array}$ & $\begin{array}{l}\text { ADDED 10/94. Parallell to Stevens } \\
\text { Drive, between N1 } 500 \text { and N2200, } \\
\text { in the paved area nearest the street }\end{array}$ & Yes & Yes \\
\hline 1100 & $\mathrm{c}$ & 484 & & $\begin{array}{l}\text { Parking area in front of gravel area that was } \\
\text { once } 1166 \text { Building - Storm water nun-olr. }\end{array}$ & $<0.50$ & Injection Well & SD2 & $\begin{array}{l}\text { E592,163.86 } \\
\text { N124,157.59 }\end{array}$ & $\begin{array}{l}\text { ADDED 10/94. Parallell to Stevens } \\
\text { Drive, belween N1500 and N2200, } \\
\text { in the paved area nearest the street }\end{array}$ & Yes & Yes \\
\hline 1100 & C & 485 & & $\begin{array}{l}\text { Parking arta in front of gravel area that was } \\
\text { once } 1166 \text { Butilding - Storm water nun-ofr. }\end{array}$ & $<0.50$ & Injection Well & SD2 & $\begin{array}{l}\text { ES92,163.81 } \\
\text { N124,176.79 }\end{array}$ & $\begin{array}{l}\text { ADDED 10/94. Parallell to Stevens } \\
\text { Drive, between N1500 and N2200, } \\
\text { in the paved area nearest the street }\end{array}$ & Yes & Yes \\
\hline
\end{tabular}

$\begin{array}{lll}\text { Source Water Krg } & \text { A-Groundwater } & \text { Bu Surface Waltet }\end{array}$

D-Potable Water

bu Streans dischagsisg to an injection well wiftin a

d- Disposal sitc willin 300 feel of en actuveduractive ent, ditch, or tench 
Table 3-1. Miscellaneous Streams Inventory

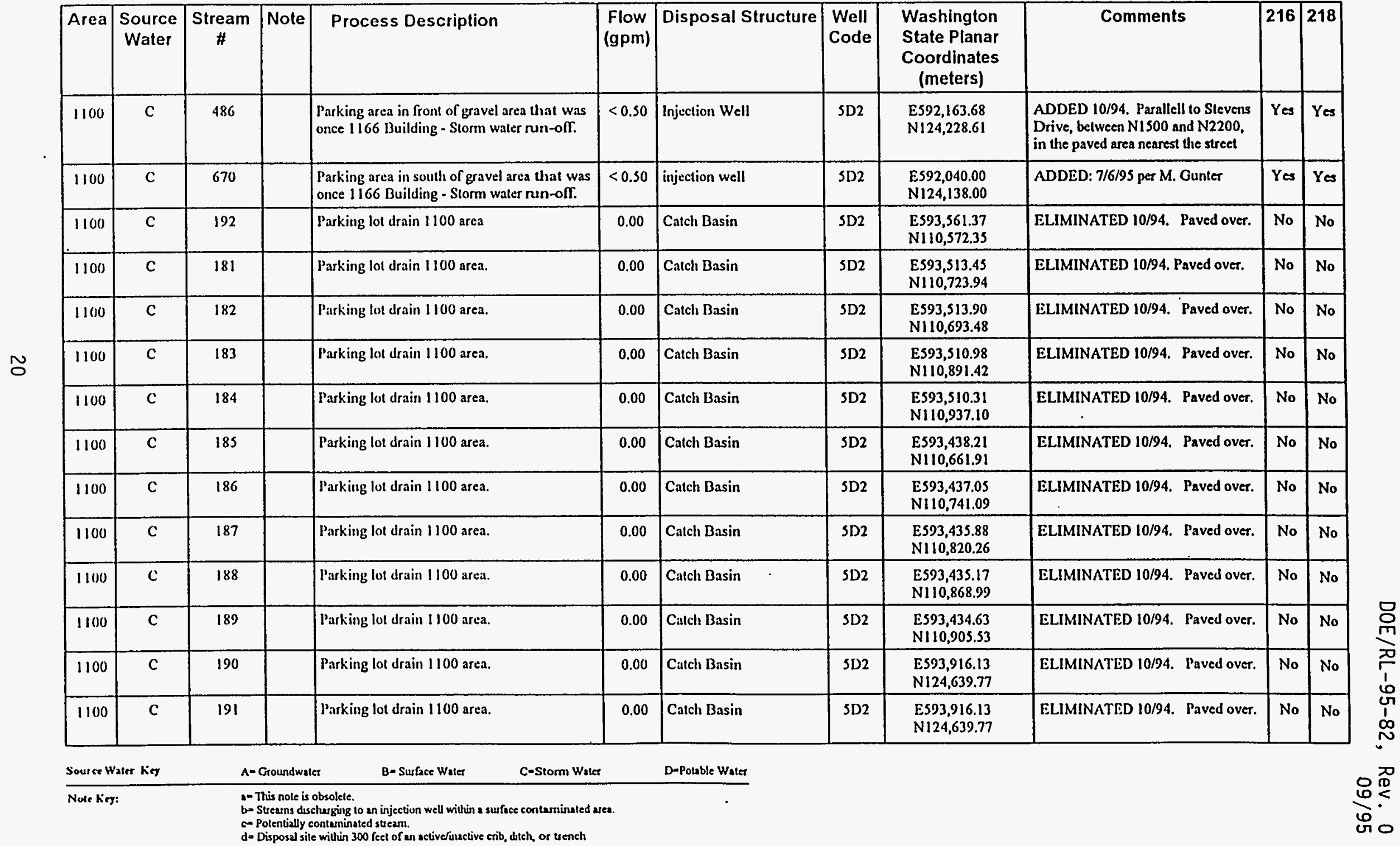


Table 3-1. Miscellaneous Streams Inventory

\begin{tabular}{|c|c|c|c|c|c|c|c|c|c|c|c|}
\hline Area & \begin{tabular}{|c|} 
Source \\
Water
\end{tabular} & $\begin{array}{c}\text { Stream } \\
\#\end{array}$ & Note & Process Description & $\begin{array}{c}\text { Flow } \\
(\text { gpm })\end{array}$ & Disposal Structure & \begin{tabular}{|l|} 
Well \\
Code
\end{tabular} & $\begin{array}{c}\text { Washington } \\
\text { State Planar } \\
\text { Coordinates } \\
\text { (meters) } \\
\end{array}$ & Comments & 216 & 218 \\
\hline 1100 & c & 193 & & Parking lot drain 1100 area. & 0.00 & Catch Basin & SD2 & $\begin{array}{l}E 593,582.69 \\
\text { N110,572.66 } \\
\end{array}$ & ELIMINATED 10/94. Paved over. & No & No \\
\hline 1100 & c & 194 & & Parking lot drain 1100 area. & 0.00 & Catch Basin & 5D2 & $\begin{array}{l}\text { E593,582.46 } \\
\text { N110,587.89 } \\
\end{array}$ & ELIMINATED 10/94. Paved over. & No & No \\
\hline 1100 & c & 195 & & Parking lot drain 1100 area. & 0.00 & Catch Basin & 5D2 & $\begin{array}{l}\text { ES93,635.42 } \\
\text { N110,611.51 } \\
\end{array}$ & ELIMINATED 10/94. Paved over. & No & No \\
\hline 1100 & $\mathrm{c}$ & 196 & & Parking lot drain 1100 areà. & 0.00 & Catch Basin & SD2 & $\begin{array}{l}\text { ES93,603.01 } \\
\text { N110,847.09 } \\
\end{array}$ & ELIMINATED 10/94. Paved over. & No & No \\
\hline 1100 & c & 197 & & P'arking lot drain 1100 area. & 0.00 & Catch Basin & SD2 & $\begin{array}{l}\text { ES93,542.11 } \\
\text { N110,846.19 } \\
\end{array}$ & |ELIMINATED 10/94. Paved over. & No & No \\
\hline 1100 & c & 198 & & Parking lot drain 1100 area. & 0.00 & Catch Basin & SD2 & $\begin{array}{l}\text { Es93,639.98 } \\
\text { N110,611.58 }\end{array}$ & ELIMINATED 10/94. Paved over. & No & No \\
\hline 200 & $\mathrm{D}$ & 168 & & $\begin{array}{l}\text { Sleam trap - 2P-Y ard-MSS-TRP-10S, 106, } \\
107 \text { - Steam condensate. }\end{array}$ & $<1.00$ & Injection Well & $5 W 20$ & $\begin{array}{l}\text { ES72,550.80 } \\
\text { Ni35,820.46 }\end{array}$ & $\begin{array}{l}\text { Ofrof steam tie-line between 200E to } \\
200 \mathrm{~W} \text {. }\end{array}$ & No & Yes \\
\hline 200 & $\mathrm{D}$ & 167 & & $\begin{array}{l}\text { Steam trap - 2P-Yard-MSS-TRP-108, } 109 \\
\text { (formerly TLT-08, 09) - Steam condensale. }\end{array}$ & $<1.00$ & Injection Well & $5 W 20$ & $\begin{array}{l}\text { ES71,904.69 } \\
\text { N135,818.63 }\end{array}$ & $\begin{array}{l}\text { Orf of steam tie-line between } 200 \mathrm{E} \text { to } \\
200 \mathrm{~W} \text {. }\end{array}$ & No & Yes \\
\hline 200 & D & 166 & & $\begin{array}{l}\text { Stean trap - 2P.Yard-MSS-TRP-110, } 111, \\
112 \text { (formerly TLT-10, 11, 12) - Steam } \\
\text { condensate. }\end{array}$ & $<1.00$ & Injection Well & $5 W 20$ & $\begin{array}{l}\text { Es71,179.35 } \\
\text { N135,816.58 }\end{array}$ & $\begin{array}{l}\text { Orf of steam tic-line belween } 200 E \text { to } \\
200 \mathrm{w} \text {. }\end{array}$ & No & Yes \\
\hline 200 & D & 165 & & $\begin{array}{l}\text { Steam trap - 21-Y Yard-MSS-TRP-113, 114, } \\
115,116 \text { (fomerly TLT-13, 14, 15, 16)- } \\
\text { Steam condensate. }\end{array}$ & $<1.00$ & Injection Well & $5 W 20$ & $\begin{array}{l}\text { ES70,539.40 } \\
\text { N135,814.79 }\end{array}$ & $\begin{array}{l}\text { Orf of steam tie-line between } 200 \mathrm{~F} \text { to } \\
200 \mathrm{~W} \text {. }\end{array}$ & No & Yes \\
\hline 200 & D & 164 & & $\begin{array}{l}\text { Steam trap - 2P-Yard-MSS-TRP-117, } 118 \\
\text { (formerly TLT-17, 18)-Steam condensate. }\end{array}$ & $<1.00$ & Injection Well & $5 W_{20}$ & $\begin{array}{l}E 569,731.57 \\
\text { N135,812.76 } \\
\end{array}$ & $\begin{array}{l}\text { Ofr of steam tie-line between } 200 \mathrm{E} \text { to } \\
200 \mathrm{~W} \text {. }\end{array}$ & No & Yes \\
\hline 200 & D & 163 & & $\begin{array}{l}\text { Steam trap - 2P.Y Yard-MSS-TRPP-119, 120, } \\
121 \text { (formerly TLT-19, 20, 21) - Steam } \\
\text { condensate. }\end{array}$ & $<1.00$ & Injection Well & $5 W 20$ & $\begin{array}{l}\text { ES69,122.07 } \\
\text { N135,811.24 }\end{array}$ & $\begin{array}{l}\text { CORRECTED: 7/6/95 per ce:mail } \\
\text { from M. Gunter. Ofr of steam tie- } \\
\text { line between 200E to } 200 \mathrm{~W} \text {. }\end{array}$ & No & Yes \\
\hline 200 & $\mathrm{D}$ & 162 & & $\begin{array}{l}\text { Steam trap - 2P-Yard-MSS-TRP-122, } 123 \\
\text { (formerly TLT-22, 23) - Steam condensate. }\end{array}$ & $<1.00$ & Injection Well & $5 W_{20}$ & $\begin{array}{l}\text { ES } 588,405.91 \\
\text { N135,809.45 }\end{array}$ & $\begin{array}{l}\text { CORRECTED: } 7 / 6 / 95 \text { per ce:mail } \\
\text { from M. Gunter. Ofr of steam tie- } \\
\text { line between 200E: to } 200 \mathrm{~W} \text {. }\end{array}$ & No & Yes \\
\hline
\end{tabular}


Table 3-1. Miscellaneous Streams Inventory

\begin{tabular}{|c|c|c|c|c|c|c|c|c|c|c|c|}
\hline Area & $\begin{array}{l}\text { Source } \\
\text { Water }\end{array}$ & $\begin{array}{c}\text { Stream } \\
\#\end{array}$ & Note & Process Description & $\begin{array}{c}\text { Flow } \\
\text { (gpm) }\end{array}$ & Disposal Structure & $\begin{array}{l}\text { Well } \\
\text { Code }\end{array}$ & $\begin{array}{l}\text { Washington } \\
\text { State Planar } \\
\text { Coordinates } \\
\text { (meters) }\end{array}$ & Comments & 216 & 218 \\
\hline 200 & $\mathrm{D}$ & 161 & & $\begin{array}{l}\text { Steam trap - 2Q-Yard-MSS-TRP-124, 125, } \\
126,127,128 \text { (fornerly TLT-24, 25, 26, } \\
27,28 \text { ) - Steam condensate. } \\
\end{array}$ & $<1.00$ & Injection Well & sw20 & $\begin{array}{l}\text { ES567.672.64 } \\
\text { N135,953.65 }\end{array}$ & $\begin{array}{l}\text { Orf of steam tie-line between } 200 \mathrm{E} \text { to } \\
200 \mathrm{~W} \text {. }\end{array}$ & No & Yes \\
\hline 2001: & D & 686 & & $\begin{array}{l}\text { 105A Mock Tank Test Sile - Electrical } \\
\text { Resistance Tomography Testing }\end{array}$ & 0.00 & non-engineered structure & & E0.00 N0.00 & $\begin{array}{l}\text { Two month duration, 8/1/95. } \\
9 / 30 / 95 \text {, of a total } 20,000 \text { gallons. }\end{array}$ & No & No \\
\hline 2001: & B & 459 & & 202A Building - PUREX pump seal water. & $<0.10$ & Injection Well (D) & $5 \wedge 19$ & $\begin{array}{l}\text { Es75,521.58 } \\
\text { N136,081.82 }\end{array}$ & $\begin{array}{l}\text { ADDED 10/94. Southwest corner of } \\
\text { 202A PUREX. }\end{array}$ & Yes & Yes \\
\hline 2001 & c & 464 & bd & $\begin{array}{l}\text { 202A Building - PUREX storm water, } \\
\text { discharged into a contaminated area. }\end{array}$ & $<0.10$ & Injection Well (K) & SD4 & $\begin{array}{l}\text { E575,200.68 } \\
\text { N135,533.58 }\end{array}$ & $\begin{array}{l}\text { ADDED 10/94. South side of } 202 \mathrm{~A} \\
\text { across the access road from } 291 \mathrm{~A} \\
\text { exhaust fans. }\end{array}$ & Yes & Yes \\
\hline 2001: & $\mathrm{C}$ & 460 & & 202A Building - PURLX storm water. & $<0.10$ & Injection Well (E) & SD2 & $\begin{array}{l}\text { E574,954.52 } \\
\text { N135,607.87 }\end{array}$ & $\begin{array}{l}\text { ADDED 10/94. Southwest comer of } \\
\text { 202A PUREX. }\end{array}$ & Yes & Yes \\
\hline $200 \mathrm{E}$ & c & 461 & d & $202 \wedge$ Building - PUREX storm water. & $<0.10$ & Injection Well (F) & SD2 & $\begin{array}{l}\text { E575,064.15 } \\
\text { N135,527.72 }\end{array}$ & $\begin{array}{l}\text { ADDED 10/94. South side of } 202 \mathrm{~A} \\
\text { PUREX, connected to the } \\
\text { proportional sample pit H04. }\end{array}$ & Yes & Yes \\
\hline 2001: & $\mathrm{c}$ & 462 & & $202 A$ Building - PUREX stom water. & $<0.10$ & Injection Well (II) & SD2 & $\begin{array}{l}\text { ES75,095.96 } \\
\text { N135,596.68 }\end{array}$ & $\begin{array}{l}\text { ADDED 10/94. South side of } 202 \mathrm{~A} \\
\text { connected to the vacuum cleaner } \\
\text { filter pit. }\end{array}$ & Yes & Yes \\
\hline 2001: & $\mathrm{c}$ & 463 & & $202 \AA$ Building - PUREX storm water. & $<0.10$ & Injection Well (I) & SD2 & $\begin{array}{l}\text { Es75,106.26 } \\
\text { N135,619.57 }\end{array}$ & $\begin{array}{l}\text { ADDED 10/94. South side of } 202 \mathrm{~A}, \\
\text { connected to steam trap pil H03. Trap } \\
\text { pit H03 is attached. approximately } \\
\text { half way down the south wall of } \\
202 \mathrm{~A} \text {. }\end{array}$ & Yes & Yes \\
\hline 2001: & $\mathrm{C}$ & 465 & b & $202 A$ Building - PUREX storn waler. & $<0.10$ & Injection Well (P) & 5D4 & $\begin{array}{l}\text { E.575,242.50 } \\
\text { N135,619.95 }\end{array}$ & $\begin{array}{l}\text { ADDED 10/94. South side of } 202 \AA \\
\text { connected to steam pit } H 1 \text {. Trap pit } \\
111 \text { is altached to the cast end of the } \\
\text { south wall of } 202 \AA \text {. }\end{array}$ & Yes & Yes \\
\hline 2001: & $\mathrm{C}$ & 466 & b & 202A lituilding - PURL:X stonn waler. & $<0.10$ & Injection Well (R) & SD4 & $\begin{array}{l}\text { EST5,274.50 } \\
\text { N135,620.03 }\end{array}$ & $\begin{array}{l}\text { ADDEDD 10/94. South side of } 202 \AA \\
\text { adjacent to the southeast comer of } \\
202 \AA\end{array}$ & Yes & Yes \\
\hline \multicolumn{12}{|c|}{ Sourre Water Keg. } \\
\hline Note kis & & & 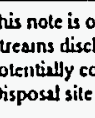 & 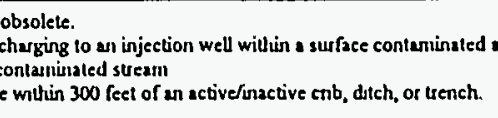 & & & & & & & \\
\hline
\end{tabular}


Table 3-1. Miscellaneous Streams Inventory

\begin{tabular}{|c|c|c|c|c|c|c|c|c|c|c|c|}
\hline Area & $\begin{array}{l}\text { Source } \\
\text { Water }\end{array}$ & $\begin{array}{c}\text { Stream } \\
\#\end{array}$ & Note & Process Description & $\begin{array}{l}\text { Flow } \\
\text { (gpm) }\end{array}$ & Disposal Structure & $\begin{array}{l}\text { Well } \\
\text { Code }\end{array}$ & $\begin{array}{l}\text { Washington } \\
\text { State Planar } \\
\text { Coordinates } \\
\text { (meters) }\end{array}$ & Comments & 216 & 218 \\
\hline 2001: & $\mathbf{C}$ & 467 & & $202 \mathrm{~A}$ Building - PUREX storm water. & 0.00 & Injection Well & SD2 & $\begin{array}{l}\text { E575,055.56 } \\
\text { N135,659.96 }\end{array}$ & $\begin{array}{l}\text { ADDED 10/94. North side of } 202 \mathrm{~A} \text {, } \\
\text { at the northwest comer of the } \\
\text { entrance walkway and the service } \\
\text { road. }\end{array}$ & Yes & Yes \\
\hline 2001: & D & 63 & bed & $\begin{array}{l}\text { 202A PURl:X - Line } 18801 \text {. Steam } \\
\text { condensate discharges to a french drain } \\
\text { located within a surface contaminated area. }\end{array}$ & 0.04 & Injection Well (O) & $5 W 20$ & $\begin{array}{l}\text { E575, } 244.18 \\
\text { N135,562.96 }\end{array}$ & $\begin{array}{l}\text { South side of 202A, SW of } 295 A \\
\text { ASD. }\end{array}$ & No & Yes \\
\hline 200r: & D & 61 & bd & $\begin{array}{l}\text { 202A PUREX - Line } \# 8801 \text {. Steam } \\
\text { condensate discharges to a french drain } \\
\text { localed within a surface contaminated area. }\end{array}$ & 0.04 & Injection Well (M) & $5 W 20$ & $\begin{array}{l}\text { ES75, } 184.63 \\
\text { N135,606.98 }\end{array}$ & $\begin{array}{l}\text { South side of } 202 \mathrm{~A} \text {, between } 202 \mathrm{~A} \\
\text { south wall and the } 291 \mathrm{AHI} \text { ammonia } \\
\text { off-gas filler building. }\end{array}$ & No & Yes \\
\hline 2001: & D & 66 & bd & $\begin{array}{l}202 A \text { PUREX - line } 18801 \text {. Steam } \\
\text { condensate discharges to a french drain } \\
\text { located within a surface contaminated area. }\end{array}$ & 0.04 & Injection Well (T) & $5 W 20$ & $\begin{array}{l}\text { ES75,283.53 } \\
\text { N135,658.46 }\end{array}$ & $\begin{array}{l}\text { North side of } 202 \mathrm{~A} \text {, near the entrance } \\
\text { to the storage tunnels. }\end{array}$ & No & Yes \\
\hline 2001: & D & 494 & & $\begin{array}{l}\text { 202A PUREX - 202- } A-417 \text { Catch tank } \\
\text { leaked steam condensale until two years ago. } \\
\text { Stream discharges to the ground. }\end{array}$ & $<0.01$ & Discharges to the ground & $N / A$ & $\begin{array}{l}\text { ES75, } 141.94 \\
\text { N135,614.49 }\end{array}$ & $\begin{array}{l}\text { ADDED I1/94. Along south wall of } \\
\text { 202A PUREX Building. }\end{array}$ & Yes & No \\
\hline 2001: & D & 62 & led & $\begin{array}{l}\text { 202A PUREX - Line } H 8801 \text {. Steam } \\
\text { condensate discharges to a french drain } \\
\text { located widlin a surface contaminated area. }\end{array}$ & 0.04 & lnjection Well (N) & $5 W 20$ & $\begin{array}{l}\text { E575,244.21 } \\
\text { N135,551.99 }\end{array}$ & $\begin{array}{l}\text { South side of } 202 \AA \text { between } 292 \mathrm{AB} \\
\text { Main Stack Building and } 218-\mathrm{E}-14 \\
\text { storage tunnel. }\end{array}$ & No & Yes \\
\hline 2001: & $\mathbf{D}$ & 64 & bd & $\begin{array}{l}\text { 202A PURl:X - Line } \$ 8801 \text {. Steam } \\
\text { condensate discharges to a french drain } \\
\text { localed within a surface contaminated area. }\end{array}$ & 0.04 & Injection Well (Q) & $5 W 20$ & $\begin{array}{l}\text { E.575,271.50 } \\
\text { N135,601.13 }\end{array}$ & $\begin{array}{l}\text { South side of } 202 \mathrm{~A} \text {, on the east side } \\
\text { of the } 218 . \mathrm{E}-15 \text { storage tunnel. }\end{array}$ & No & Yes \\
\hline 2001: & D & $65^{\circ}$ & b & $\begin{array}{l}202 \text { A PUREX - Line } 18801 \text {. Steam } \\
\text { condensate. }\end{array}$ & 0.04 & Injection Well (S) & SW20 & $\begin{array}{l}\text { E575,274.12 } \\
\text { N135,645.33 }\end{array}$ & $\begin{array}{l}\text { North side of } 202 \mathrm{~A} \text {, along the cast } \\
\text { side of the north wall next to the } 218- \\
\text { E-14 storage tunnel. }\end{array}$ & No & Yes \\
\hline 2001: & D & 56 & & $\begin{array}{l}202 \mathrm{~A} \text { PURLI:X - Sleam condensale line } \\
\text { HI801. }\end{array}$ & 0.10 & Injection Well $(\Lambda)$ & $5 W 20$ & $\begin{array}{l}\text { E574,933.29 } \\
\text { N135,718.59 }\end{array}$ & $\begin{array}{l}\text { N.W. comer of PURR:X where the } \\
\text { stean line enters through the security } \\
\text { fences }\end{array}$ & No & Yes \\
\hline 2001: & D & 57 & d & $\begin{array}{l}202 \mathrm{~A} \text { PURREX - Stcam condensale line } \\
118801 .\end{array}$ & 0.04 & Injection Well (B) & $5 W 20$ & $\begin{array}{l}\text { ES74,933.17 } \\
\text { N135,613.91 }\end{array}$ & West sille of PUREX. & No & Yes \\
\hline
\end{tabular}

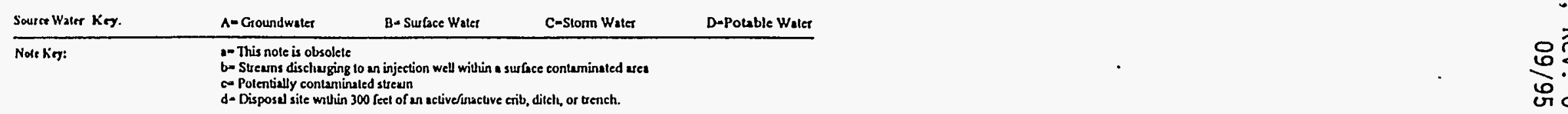


Table 3-1. Miscellaneous Streams Inventory

\begin{tabular}{|c|c|c|c|c|c|c|c|c|c|c|c|}
\hline Area & $\begin{array}{l}\text { Source } \\
\text { Water }\end{array}$ & $\begin{array}{c}\text { Stream } \\
\#\end{array}$ & Note & Process Description & $\begin{array}{c}\text { Flow } \\
(\mathrm{gpm})\end{array}$ & Disposal Structure & $\begin{array}{l}\text { Well } \\
\text { Code }\end{array}$ & $\begin{array}{l}\text { Washington } \\
\text { State Planar } \\
\text { Coordinates } \\
\text { (meters) }\end{array}$ & Comments & 216 & 218 \\
\hline 2001: & D & 60 & d & $\begin{array}{l}\text { 202A PUUREX - Steam condersate line } \\
\text { H8801. }\end{array}$ & 0.04 & Injection Well (G) & $5 W 20$ & $\begin{array}{l}\text { Es75, } 105.64 \\
\text { Ni3s, } 514.72\end{array}$ & $\begin{array}{l}\text { South side of PUREX, prior to the } \\
\text { H04 gate access. }\end{array}$ & No & Yes \\
\hline 2001: & D) & 67 & d & $\begin{array}{l}\text { 202A PUREX - Steam condersate line } \\
\text { \#8801. }\end{array}$ & 0.04 & Injection Well (U) & $5 W 20$ & $\begin{array}{l}\text { E575,283.73 } \\
\text { N135,902.83 }\end{array}$ & $\begin{array}{l}\text { North comer of PUREX, in the } \\
\text { exclusion zone. }\end{array}$ & No & Yes \\
\hline 2001: & $\mathrm{D}$ & 68 & d & $\begin{array}{l}202 \AA \text { PUREX - Steam condensate line } \\
\text { H8801. }\end{array}$ & 0.04 & Injection Well (V) & $5 W 20$ & $\begin{array}{l}\text { ES75,222.28 } \\
\text { N135,655.85 }\end{array}$ & $\begin{array}{l}\text { North side of } 202 \mathrm{~A} \text {, next to the north } \\
\text { wall of } 206 \mathrm{~A} \text { fractionator. }\end{array}$ & No & Yes \\
\hline 2001: & D & 69 & d & $\begin{array}{l}\text { 202A PUREX - Sleam condensale line } \\
\text { H8801. }\end{array}$ & 0.04 & Injection Well (W) & $5 W 20$ & $\begin{array}{l}\text { ES75,136.01 } \\
\text { N135,662.32 }\end{array}$ & $\begin{array}{l}\text { North side of } 202 \mathrm{~A} \text {, on the west wall } \\
\text { of the laboratory sample receiving } \\
\text { dock. }\end{array}$ & No & Yes \\
\hline 2001: & $\mathrm{D}$ & 70 & d & $\begin{array}{l}\text { 202A PURREX - Steam condensate line } \\
\text { H8801. }\end{array}$ & 0.04 & Injection Well $(Y)$ & $5 W 20$ & $\begin{array}{l}\text { ES75, } 118.24 \\
\mathrm{~N} 135,735.28\end{array}$ & $\begin{array}{l}\text { North side of } 202 \mathrm{~A} \text {, between 203A } \\
\text { UNH1 pumphouse and MO332. }\end{array}$ & No & Yes \\
\hline $2001:$ & D & 71 & d & $\begin{array}{l}\text { 202A PUREX - Steam condensate line } \\
\text { H8801. }\end{array}$ & 0.04 & Injection Well (Z) & $5 W 20$ & $\begin{array}{l}\text { ES75,108.20 } \\
\text { N135,797.01 }\end{array}$ & $\begin{array}{l}\text { North of } 202 \mathrm{~A} \text {, on the north side of } \\
\text { TK-P3 containment dike, in the } \\
\text { exclusion zone. }\end{array}$ & No & Yes \\
\hline $200 E$ & D & 72 & d & $\begin{array}{l}\text { 202A PUREX - Steam condensale line } \\
\text { H880 } 1 \text {. }\end{array}$ & 0.04 & Injection Well (AA) & $5 W 20$ & $\begin{array}{l}\text { ES75,076.69 } \\
\text { N135,716.87 }\end{array}$ & $\begin{array}{l}\text { North side of } 202 \mathrm{~A} \text {, on the NW } \\
\text { cormer of } 211 \mathrm{~A} \mathrm{storage} \mathrm{tanks,} \\
\text { between TK }-41 \text { and } 211 \mathrm{~A} \text { chemical } \\
\text { access track. }\end{array}$ & No & Yes \\
\hline 2001 & $\mathrm{CD}$ & 58 & & $\begin{array}{l}\text { 202A rURrEX - Steam condensate, also has } \\
\text { potential to receive storm water. }\end{array}$ & 0.04 & Injection Well (C) & SD2, & $\begin{array}{l}\text { Es74,959.05 } \\
\text { N135,624.34 }\end{array}$ & $\begin{array}{l}\text { West side of } 202 \AA \text {, south of the PR- } \\
\text { Dock. }\end{array}$ & Yes & Yes \\
\hline 2001: & $C D$ & 59 & bd & $\begin{array}{l}\text { 202A PUREX - Stomm water potentially } \\
\text { discharges to a french drain localed within } \\
\text { surface contaminated area. Drain was used } \\
\text { to collect steam condensale from the } 291 \wedge \text {. } \\
\text { control house. }\end{array}$ & 0.04 & Injection Well (L) & SD4 & $\begin{array}{l}\text { ES75,212.20 } \\
\text { N135,555.86 }\end{array}$ & $\begin{array}{l}\text { South side of } 202 \mathrm{~A}, \text { between } 291 \AA \\
\text { exhaust fans and } 292 \mathrm{AB} \text { Main Stack } \\
\text { Building. }\end{array}$ & Yes & Yes \\
\hline $200 E$ & D & 657 & d & 204-AR Steam Trap & 0.00 & Injection Well & $5 \mathrm{~W} 20$ & $\begin{array}{l}\text { ES75,186.00 } \\
\text { Ni36,010.00 } \\
\end{array}$ & Added 6/95. Currently inactive & No & Yes \\
\hline 200E: & D & 316 & & $\begin{array}{l}2101 \mathrm{M} \text { Building - Steam condensate, batch } \\
\text { discharge during winter, northeast side. }\end{array}$ & 0.00 & Injection Well & 5620 & $\begin{array}{l}\text { ES73,639.42 } \\
\mathrm{N} 135,397.48 \\
\end{array}$ & ELIMINATED 10/94 & No & No \\
\hline Sourre W & Vater Kers & & oundwate & B- Sufface Wattr & & Dopolable Water & & & & & \\
\hline
\end{tabular}


Table 3-1. Miscellaneous Streams Inventory

\begin{tabular}{|c|c|c|c|c|c|c|c|c|c|c|c|}
\hline Area & $\begin{array}{l}\text { Source } \\
\text { Water }\end{array}$ & $\begin{array}{c}\text { Stream } \\
\#\end{array}$ & Note & Process Description & $\begin{array}{c}\text { Flow } \\
\text { (gpm) }\end{array}$ & Disposal Structure & \begin{tabular}{|l|} 
Well \\
Code
\end{tabular} & $\begin{array}{l}\text { Washington } \\
\text { State Planar } \\
\text { Coordinates } \\
\text { (meters) } \\
\end{array}$ & Comments & 216 & 218 \\
\hline 2001: & D & 220 & $b$ & $\begin{array}{l}241 \text { A Tank Farm Steam Condensale - Steam } \\
\text { condensate is dischirged year-round to a } \\
\text { caisson located in a surface contaminated } \\
\text { area. }\end{array}$ & $<5.00$ & Injection Well & $5 w_{20}$ & $\begin{array}{l}\text { Es75,361.38 } \\
\text { N136, } 145.99\end{array}$ & $\begin{array}{l}\text { CORRECTED 2/95. Contact and } \\
\text { drawing corrected. Will be } \\
\text { eliminated by 12/96. Under the over } \\
\text { ground steam line between AY- Farm } \\
\text { and } 241-A-702 \text {. }\end{array}$ & No & Yes \\
\hline 2001: & D & 218 & b & \begin{tabular}{|l|}
$241 \mathrm{AZ}$ Tank Farm, AZ-154 Steam \\
Condenstate Catch Basin - Steam condensate \\
is discharged year-round to a caisson located \\
in a surface contaminated area.
\end{tabular} & $<5.00$ & Injection Well & $5 w_{20}$ & $\begin{array}{l}\text { ES75,440.26 } \\
\text { N136,270.59 }\end{array}$ & $\begin{array}{l}\text { ELIMINATED 4/95. Does not } \\
\text { discharge to ground Will reroute to } \\
\text { the Aging Waste tanks by } 5 / 96 \text {. Just } \\
\text { inside AZ Tank Farm perimeler } \\
\text { fence, southeast of tank } 101 \mathrm{AZ} \text {. }\end{array}$ & No & No \\
\hline 2001: & D) & 547 & d & $\begin{array}{l}242 \Lambda \text { Building - Injection wells receives } \\
\text { eflluent from the } 242-A-2,242-A-3,242 \text {. } \\
A-4 \text { stean traps, and PRV }-E A 1-1 . .\end{array}$ & $<1.00$ & Injection Well & sw20 & $\begin{array}{l}\text { ES75,374.09 } \\
\text { N135,954.68 }\end{array}$ & $\begin{array}{l}\text { CORRECTED 7/6/95 PER } \\
\text { CC:MAALL from Mark Bowman } \\
\text { dated 7/5/95. (stream \#562, 548, } \\
\text { currenlly deleted from inventory, } \\
\text { discharge into stream \#547). Added } \\
\text { 2/95. }\end{array}$ & No & Yes \\
\hline 2001: & D & 118 & & $\begin{array}{l}242 \mathrm{~A} \text { Building. Injection well receives } \\
\text { sleam condensale from two steam traps and a } \\
\text { relief valve (PSV.EAI - } 1 \text { ). }\end{array}$ & 0.00 & . & & $\begin{array}{l}\text { Es75,374.09 } \\
\text { N135,954.68 }\end{array}$ & $\begin{array}{l}\text { ELIMINATED: same stream as \# } \\
\text { S47 per Bowman 8/22/95. } \\
\text { CORRECTED: 7/6/95 per cc:mail } \\
\text { from Mark Bowman dated 7/5/95. } \\
\text { (stream H1 19, currently deleted from } \\
\text { inventory, discliarges to stream H118) } \\
\text { CORRECTED } 2 / 95 \text {. SW of } 242 \AA \\
\text { Evaporator }\end{array}$ & No & No \\
\hline 2001: & $\mathrm{D}$ & so & d & 242AC Pipefilter's Shop, steam trap & 0.05 & Injection Well & $5 w_{20}$ & $\begin{array}{l}\text { ES75,296.75 } \\
\text { N135,968.37 }\end{array}$ & $\begin{array}{l}\text { CORRECTED 7/6/95 PER ce:MAIL } \\
\text { from M. Gunter dated } 6 / 28 / 95 \text {. } \\
\text { INACTIVE } 5 / 95 \text {. } 242 \wedge C \text { No longer } \\
\text { receives steam. Stream is localed I } \\
\text { foot from stream } \# 48 \text {. }\end{array}$ & No & Yes \\
\hline 2001: & D & 48 & d & $\begin{array}{l}\text { 242AC Pipefitter's Shop: duret discharge } \\
\text { lines to disposal site (242AC steam } \\
\text { condensate, pipefitter storage skid slack } \\
\text { condensale, strean HSO steann trap). }\end{array}$ & $<0.05$ & Injection Well & $5 \mathrm{~W} 20$ & $\begin{array}{l}\text { E575,295.75 } \\
\text { N135,968.37 }\end{array}$ & $\begin{array}{l}\text { CORRRCCTED: 7/6/95 per CC:Mail } \\
\text { from M. Gunter dated 6/29/95. } \\
\text { INAC'TIVE 5/95. 242AC no longet } \\
\text { receives steam. }\end{array}$ & No & Yes \\
\hline
\end{tabular}


Table 3-1. Miscellaneous Streams Inventory

\begin{tabular}{|c|c|c|c|c|c|c|c|c|c|c|c|}
\hline Area & $\begin{array}{l}\text { Source } \\
\text { Water }\end{array}$ & $\begin{array}{c}\text { Stream } \\
\#\end{array}$ & Note & Process Description & $\begin{array}{c}\text { Flow } \\
\text { (gpm) }\end{array}$ & Disposal Structure & $\begin{array}{l}\text { Well } \\
\text { Code }\end{array}$ & $\begin{array}{c}\text { Washington } \\
\text { State Planar } \\
\text { Coordinates } \\
\text { (meters) }\end{array}$ & Comments & 216 & 218 \\
\hline 200E & $D$ & 452 & & 2704IIV Building - Steam condensate. & $<0.01$ & Injection Well & sw20 & $\begin{array}{l}\text { ES72,609.65 } \\
\text { N136,579.50 }\end{array}$ & $\begin{array}{l}\text { ADDED 2/95. } 150 \text { feel south of } \\
270411 V \text { towards southwest comer. }\end{array}$ & No & Yes \\
\hline 200E & D & 563 & & 2704IIV Building - Steam condensate. & $<0.01$ & Injection Well & $5 w 20$ & $\begin{array}{l}E 572,625.50 \\
N 136,579.54\end{array}$ & $\begin{array}{l}\text { ADDED 2/95. } 150 \text { feet south of } \\
270411 \mathrm{~V} \text {, towards southeast comer. }\end{array}$ & No & Yes \\
\hline 2001: & c & 564 & & 2704IIV Building stomwwater nunoff & $<5.00$ & Collection Basin & $\mathrm{N} / \mathrm{A}$ & $\begin{array}{l}E 572,552.71 \\
\text { N136,825.96 }\end{array}$ & $\begin{array}{l}\text { ADDED 2/95. North of } 270411 \mathrm{~V} \text {, } \\
400 \text { feet from northwest comer. }\end{array}$ & Yes & No \\
\hline $200 \mathrm{E}$ & $\mathrm{C}$ & $\$ 30$ & & 270alIV Building stormwater nunofi: & $<5.00$ & Collection Basin & N/A & $\begin{array}{l}\text { ES72,464.30 } \\
\text { N136,825.70 }\end{array}$ & $\begin{array}{l}\text { ADDED 2/95. Northwest of } \\
2704 \mathrm{HV} \text { at north end of parking lot. }\end{array}$ & Yes & No \\
\hline 2001: & $\mathrm{D}$ & 488 & & $\begin{array}{l}\text { 2707E Building - Steam condensate hatch } \\
\text { discharge during winter. }\end{array}$ & $<5.00$ & Injection Well & $5 W 20$ & $\begin{array}{l}\text { ES73,548.99 } \\
\text { N135,719.88 }\end{array}$ & $\begin{array}{l}\text { ADDED 10/94. Southwest side of } \\
\text { 2707-E Building }\end{array}$ & No & Yes \\
\hline 2001: & D & 312 & & $\begin{array}{l}\text { 2707E Building - Steam condensale north } \\
\text { side, balch discharge during winter. }\end{array}$ & $<5.00$ & Injection Well & sw20 & $\begin{array}{l}\text { ES73,572.15 } \\
\text { N135,729.31 }\end{array}$ & $\begin{array}{l}\text { CORRECTED 7/94. Disposal } \\
\text { Structure Changed }\end{array}$ & No & Yes \\
\hline 200r: & $\mathrm{D}$ & 522 & & 2707E Building - Steam condensale. & $<1.00$ & Injection Well & $5 w 20$ & $\begin{array}{l}\text { Es73,574.15 } \\
\text { N135,719.59 }\end{array}$ & $\begin{array}{l}\text { ADDED 2/93. 2707E southeast } \\
\text { comer. }\end{array}$ & No & Yes \\
\hline $200 \mathrm{E}$ & D & 313 & & $\begin{array}{l}\text { 2713E Building - Steam condensale, batch } \\
\text { discharged during winter. }\end{array}$ & $<5.00$ & Injection Well & $5 W 20$ & $\begin{array}{l}\text { Es73,612.09 } \\
\text { N135,555.69 }\end{array}$ & $\begin{array}{l}\text { CORRECTED 2/95. Coordinates } \\
\text { and comments changed. } \\
\text { CORRECTED 7/94. Disposal } \\
\text { Structure Changed. Southwest } \\
\text { comer of 2713E. }\end{array}$ & No & Yes \\
\hline 200: & D & 92 & & $\begin{array}{l}\text { 2715E Building - Steam Trap } 1102 \text { - This } \\
\text { steam trap is on the line to the building, past } \\
\text { the first cutof from the main leader. }\end{array}$ & $<1.00$ & Injection Well & SW20 & $\begin{array}{l}\text { ES73,670.56 } \\
\text { N135,559.71 }\end{array}$ & South of $2715 E$ & No & $\overline{\text { Yes }}$ \\
\hline 2001: & D & 93 & & $\begin{array}{l}\text { 27ISE Building - Steain Trap H103 - This } \\
\text { steam Irap is on the line to the building. past } \\
\text { the first cutoff from the main header. }\end{array}$ & $<1.00$ & Injection Well & sw20 & $\begin{array}{l}\text { ES73,670.56 } \\
\text { N135,560.15 }\end{array}$ & South of $2715 \mathrm{E}$ & No & Yes \\
\hline 2001: & D & 94 & & $\begin{array}{l}\text { 2715l: Building - Steam Trap } / 104 \text { - This } \\
\text { steam trap is on the line to the building, past } \\
\text { the first cutoff from the main header. }\end{array}$ & $<1.00$ & Injection Well & $5 w 20$ & $\begin{array}{l}\text { ES73,674.41 } \\
\text { N135,569.63 }\end{array}$ & South or $2715 E$ & No & $\overline{\text { Yes }}$ \\
\hline
\end{tabular}

$\begin{array}{lll}\text { Source Water kieg } & \text { A- Groundwater } & \text { B- Surface Water }\end{array}$


Table 3-1. Miscellaneous Streams Inventory

\begin{tabular}{|c|c|c|c|c|c|c|c|c|c|c|c|}
\hline Area & $\begin{array}{l}\text { Source } \\
\text { Water }\end{array}$ & $\begin{array}{c}\text { Stream } \\
\#\end{array}$ & Note & Process Description & $\begin{array}{l}\text { Flow } \\
\text { (gpm) }\end{array}$ & Disposal Structure & $\begin{array}{l}\text { Well } \\
\text { Code }\end{array}$ & $\begin{array}{c}\text { Washington } \\
\text { State Planar } \\
\text { Coordinates } \\
\text { (meters) }\end{array}$ & Comments & 216 & 218 \\
\hline 2001: & D) & 315 & & $\begin{array}{l}\text { 27ISE Building - Steam condensate, batch } \\
\text { discharged during winter. }\end{array}$ & $<5.00$ & Injection Well & $5 W 20$ & $\begin{array}{l}E 573,721.89 \\
N 135,549.47\end{array}$ & $\begin{array}{l}\text { CORRECTED 7/94. Disposal } \\
\text { Structure Changed. South side of } \\
\text { 2715E. INACTIVE. }\end{array}$ & No & Yes \\
\hline 2001: & $\mathrm{D}$ & 489 & & $\begin{array}{l}\text { 27ISE Building - Steam condensate; batch } \\
\text { discharged during winter. }\end{array}$ & $<5.00$ & Injection Well & SW20 & $\begin{array}{l}\text { E573,655,38 } \\
\text { N135,571.42 }\end{array}$ & $\begin{array}{l}\text { ADDED 10/94. West side of 2715- } \\
\text { E building. INACTIVE }\end{array}$ & No & Yes \\
\hline 2001: & D & 223 & c & $\begin{array}{l}\text { 271SEC Building - Paint Shop sink used to } \\
\text { wash latex paint bnushes. }\end{array}$ & $<1.00$ & Injection Well & $5 W 20$ & $\begin{array}{l}\text { E573,625.04 } \\
\text { N135,661.85 }\end{array}$ & $\begin{array}{l}\text { Northeast comer of } 2715 E C 10 \text { feet } \\
\text { west } 25 \text { feet north. }\end{array}$ & Yes & Yes \\
\hline 2001: & $\mathrm{D}$ & 224 & & $\begin{array}{l}\text { 2715EC Building - Steam condensatc and } \\
\text { overilow from building heating and cooling } \\
\text { unit. }\end{array}$ & $<1.00$ & Injection Well & $5 w 20$ & $\begin{array}{l}\text { Es73,627.04 } \\
\text { N135,661.27 }\end{array}$ & $\begin{array}{l}\text { CORRECTED 11/94. Disposal } \\
\text { structure changed. Northeast comer } \\
\text { of } 2715 E C \text {. }\end{array}$ & No & Yes \\
\hline 200E: & D & $\$ 27$ & & 271 SEC Building - Steam condensale. & 1.00 & Injection Well & $5 w 20$ & $\begin{array}{l}\text { ES73,604.74 } \\
\text { N135,653.27 }\end{array}$ & $\begin{array}{l}\text { ADDED 2/95. } 20^{\circ} \text { West of the } \\
\text { southwest comer 2715EC Building }\end{array}$ & No & Yes \\
\hline 200E: & D & $\$ 28$ & & 2715EC Building - Stean condensate. & 1.00 & Injection Well & $5 W 20$ & $\begin{array}{l}\text { ES73,604.74 } \\
\text { N135,664.99 }\end{array}$ & $\begin{array}{l}\text { ADDED 2/95. 10' North and 20' } \\
\text { West of the northwest comer of } \\
\text { Building 2715EC. }\end{array}$ & No & Yes \\
\hline 200E & $\mathrm{c}$ & 529 & & 2715EC Building - Storm water nun-off. & 1.00 & Injection Well & SD2 & $\begin{array}{l}\text { ES73,614.17 } \\
\text { N135,666.42 }\end{array}$ & $\begin{array}{l}\text { ADDED 2/95. } 10^{\prime} \text { North of the } \\
\text { northwest comer of } 2715 E C \text {. }\end{array}$ & Yes & Yes \\
\hline 2001: & D & 311 & & $\begin{array}{l}\text { 2719R: Building - Stean condensatc; batch } \\
\text { discharged during winter. }\end{array}$ & $<5.00$ & Injection Well & $5 W 20$ & $\begin{array}{l}E 573,604.03 \\
\text { N135,767.19 }\end{array}$ & $\begin{array}{l}\text { CORRECTED 7/94. Disposal } \\
\text { Structure Changed. West side of } \\
2719 E \text {. }\end{array}$ & No & Yes \\
\hline $.2001:$ & $\bar{D}$ & 490 & & $\begin{array}{l}\text { 271B Building - Lunch room ice machine } \\
\text { overflow. }\end{array}$ & $<0.07$ & Injection Well & sw20 & $\begin{array}{l}\text { E.573,467.24 } \\
\text { N136,477.69 }\end{array}$ & $\begin{array}{l}\text { ADDED 7/94. Scheduled to be } \\
\text { rerouted }\end{array}$ & Yes & Yes \\
\hline 2001: & $\mathrm{c}$ & 496 & & $\begin{array}{l}\text { 2721 EA Building - Stonn water nin-off from } \\
\text { roof drains and paved surface. }\end{array}$ & $<0.01$ & Injection Well & SD2 & $\begin{array}{l}\text { E:S72,865.78 } \\
\text { N135,670.08 }\end{array}$ & $\begin{array}{l}\text { ADDISD 12/94. Approximately 15 } \mathrm{n} \\
\text { west of } 2721 \mathrm{EA}\end{array}$ & Yes & Yes \\
\hline
\end{tabular}

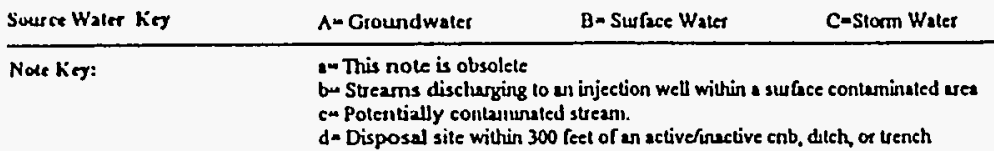

D-Potable Water 
Table 3-1. Miscellaneous Streams Inventory

\begin{tabular}{|c|c|c|c|c|c|c|c|c|c|c|c|}
\hline Area & $\begin{array}{l}\text { Source } \\
\text { Water }\end{array}$ & $\begin{array}{c}\text { Stream } \\
\#\end{array}$ & Note & Process Description & $\begin{array}{l}\text { Flow } \\
\text { (gpm) }\end{array}$ & Disposal Structure & $\begin{array}{l}\text { Well } \\
\text { Code }\end{array}$ & $\begin{array}{l}\text { Washington } \\
\text { State Planar } \\
\text { Coordinates } \\
\text { (meters) }\end{array}$ & Comments & 216 & 218 \\
\hline 2001: & $\mathbf{D}$ & 659 & & $\begin{array}{l}\text { 272BD: inactive french drain east side of } \\
\text { builidng. }\end{array}$ & 0.00 & injection well & & $\begin{array}{l}\text { Es73,508.31 } \\
\text { N136,541.48 }\end{array}$ & $\begin{array}{l}\text { ELIMINATED: } 67 / 6 / 95 \text { per cc:mail } \\
\text { from R. Weissenfels dated 7/6/95. } \\
\text { Sources include floor drain that is } \\
\text { temporily plugged and will be } \\
\text { permanently plugged and sink drain } \\
\text { that has been removed and capped. }\end{array}$ & No & No \\
\hline 2001: & $D$ & 500 & & 272E Building - Receives steam condensate. & $<0.50$ & $\begin{array}{l}\text { Injection Well, Labelled } \\
\text { :E2 }\end{array}$ & $5 W 20$ & $\begin{array}{l}\text { E573,575.99 } \\
\text { N135,647.43 }\end{array}$ & ADDED 12/94. east side of $272 E$ & No & Yes \\
\hline 2001: & $\mathrm{D}$ & 498 & & $\begin{array}{l}\text { 272E Building - IIVAC/ steam condensate } \\
\text { to an eight inch diameter french drain. }\end{array}$ & $<0.01$ & Injuction Well & $5 W 20$ & $\begin{array}{l}\text { Es73,558.64 } \\
\text { N135,619.24 }\end{array}$ & $\begin{array}{l}\text { ADDED 12/94. south side of 272E, } \\
\text { adjacent to building }\end{array}$ & No & $\overline{Y e s}$ \\
\hline 2001: & D & 501 & & 272E Building - Steam condensate. & $<0.01$ & Injection Well & sw20 & $\begin{array}{l}\text { Es73,575.55 } \\
\text { N135,634.42 }\end{array}$ & ADDED 12/94. East of 272E & No & Yes \\
\hline 200E & $\mathrm{D}$ & 310 & & $\begin{array}{l}\text { 272E Building - Sleain condensate; batch } \\
\text { discharged during winter. }\end{array}$ & $<5.00$ & Injection Well & $5 W 20$ & $\begin{array}{l}\text { ES73,561.86 } \\
\text { N135,684.14 }\end{array}$ & $\begin{array}{l}\text { CORRECTED 7/94. Disposal } \\
\text { structure changed. North side of } \\
\text { 272E. }\end{array}$ & No & Yes \\
\hline 200l: & c & 499 & & $\begin{array}{l}\text { 272E Building - Storm water from walkway. } \\
\text { Overflow from Stream } \# 500 \text {. }\end{array}$ & $<0.01$ & $\begin{array}{l}\text { Injection Well, Labelled } \\
\text { El }\end{array}$ & SD2 & $\begin{array}{l}\text { Es73,578.59 } \\
\text { N135,647.43 }\end{array}$ & $\begin{array}{l}\text { ADDED 12/94, east side of 272E, } \\
\text { adjacent to building }\end{array}$ & Yes & $\overline{Y e s}$ \\
\hline 2001: & $\mathrm{D}$ & 559 & & 272E Building - Water from valve. & $<1.00$ & Injection Well & $5 W 20$ & $\begin{array}{l}\text { E573,575.99 } \\
\text { N135,604.06 }\end{array}$ & $\begin{array}{l}\text { ADDED 2/95. } 50 \text { fect south of } \\
\text { southwest comer of } 272 E \text {. }\end{array}$ & Yes & Yes \\
\hline 2001: & $D$ & 560 & & $\begin{array}{l}\text { 273l: Iuilding } 72 \text {-inch well - Water from } \\
\text { vacuum vent line for sanilary water. }\end{array}$ & $<1.00$ & Injection Well & $5 W 20$ & $\begin{array}{l}\text { E573,476.03 } \\
\text { N135,733.16 }\end{array}$ & $\begin{array}{l}\text { ADDED 2/95. } 50 \text { feet north of 273E } \\
\text { towards west side. }\end{array}$ & Yes & $\overline{\text { Yes }}$ \\
\hline 200l: & D & $56 !$ & & $\begin{array}{l}\text { 273 E: Building Control valve above } 12 \text {-inch } \\
\text { well - Potable waler from valve. }\end{array}$ & $<1.00$ & Injection Well & $5 W 20$ & $\begin{array}{l}\text { ES53,509.71 } \\
\text { N135,732.41 }\end{array}$ & $\begin{array}{l}\text { ADDED 2/95. } 50 \text { fect north of 273E } \\
\text { towards east side. }\end{array}$ & Yes & $\overline{Y e s}$ \\
\hline 2001: & $\mathrm{D}$ & 630 & & $\begin{array}{l}\text { 2750E Building - Overllow cooling water } \\
\text { from the evaporative cooler (cools condensor } \\
\text { coils on the building heat pumps) discharges } \\
\text { to a trench the south of } 2750 \text {. }\end{array}$ & 0.13 & Trench & N/A & $\begin{array}{l}\text { EST3,596.00 } \\
\text { N135,069.00 }\end{array}$ & $\begin{array}{l}\text { ADDrED 4/95. South of } 2750 \text { E. } \\
\text { Structure is to be completed in the } \\
\text { summer of } 1995 \text {. }\end{array}$ & Yes & No \\
\hline
\end{tabular}

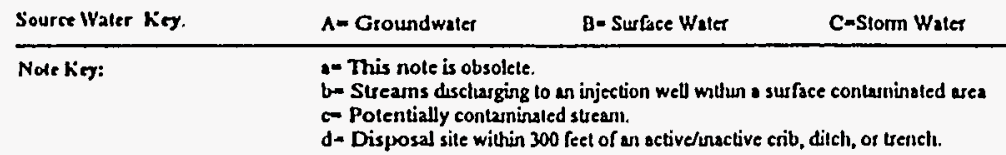

DuPolable Water 
Table 3-1. Miscellaneous Streams Inventory

\begin{tabular}{|c|c|c|c|c|c|c|c|c|c|c|c|}
\hline Area & $\begin{array}{l}\text { Source } \\
\text { Water }\end{array}$ & $\begin{array}{c}\text { Stream } \\
\#\end{array}$ & Note & Process Description & $\begin{array}{l}\text { Flow } \\
\text { (gpm) }\end{array}$ & Disposal Structure & $\begin{array}{l}\text { Well } \\
\text { Code }\end{array}$ & $\begin{array}{l}\text { Washington } \\
\text { State Planar } \\
\text { Coordinates } \\
\text { (meters) }\end{array}$ & Comments & 216 & 218 \\
\hline 2001: & D & 629 & & $\begin{array}{l}2750 \text { Building - Steam condensate from } \\
\text { building heating discharges to a trench near } \\
\text { the southwest coner of } 2750 \text {. }\end{array}$ & 0.30 & Trench & N/A & $\begin{array}{l}\text { EST5, } 164.00 \\
\text { N135,916.00 }\end{array}$ & $\begin{array}{l}\text { ADDED } 4 / 95 \text {. Southwest of } 2750 E \text {. } \\
\text { Structure is to be completed in the } \\
\text { summer of } 1995 \text {. }\end{array}$ & Yes & No \\
\hline 2001: & c & 625 & & $\begin{array}{l}2750 \text { B Building - An outfall collects } \\
\text { stormwaler from a network of catcli basins in } \\
\text { the parking area south of } 2750 \mathrm{E} \text {. The } \\
\text { outfall discharges to a gravel pad in a diech } \\
\text { southwest of } 2751 \mathrm{E} \text {. }\end{array}$ & $<0.04$ & $\begin{array}{l}\text { Outfall (discharges to a } \\
\text { ditch) }\end{array}$ & $N / A$ & $\begin{array}{l}\text { ES73,570.92 } \\
\text { N13S,048.34 }\end{array}$ & $\begin{array}{l}\text { ADDED 4/95. Off southwest corner } \\
\text { of } 2751 \text { E, actoss from road. }\end{array}$ & Yes & No \\
\hline 2001: & c & 624 & & $\begin{array}{l}\text { 275OE Building - An outfall northwest of } \\
2750 E \text { collects stormwater from a network } \\
\text { of Is calch basins and storm drain/mantholes } \\
\text { throughout the parking areas norhth of } 2750 \mathrm{E} \\
\text { and discharges to a ditch. This includes those } \\
\text { calch basins around MO-234 and MO-21. }\end{array}$ & $<0.10$ & $\begin{array}{l}\text { Outfall (discharges to a } \\
\text { ditch) }\end{array}$ & N/A & $\begin{array}{l}E 573,424.21 \\
\text { N135,195.12 }\end{array}$ & $\begin{array}{l}\text { ADDED 4/95. Outfall is located } \\
2220 \text { feet north of } 2752 E \text {. }\end{array}$ & Yes & No \\
\hline 200E: & D & 314 & & $\begin{array}{l}\text { 275E Building - Steam condensate; batch } \\
\text { discliarged during winter. }\end{array}$ & $<5.00$ & Injection Well & $5 W 20$ & $\begin{array}{l}\text { ES73,706.54 } \\
\text { N135,614.47 }\end{array}$ & $\begin{array}{l}\text { CORRECTED 7/94. Disposal } \\
\text { Structure Changed. East side of } \\
\text { 275E. }\end{array}$ & No & Yes \\
\hline 200l: & D & 525 & & 275E Building - Steam condensate. & $<1.00$ & Injection Well & $5 W 20$ & $\begin{array}{l}\text { E573,674.88 } \\
\text { N135,604.93 }\end{array}$ & $\begin{array}{l}\text { ADDED 2/95. } 10 \text { ' west of the center } \\
\text { of west side } 275 \mathrm{E} \text { Building. }\end{array}$ & No. & Yes \\
\hline $2001:$ & $\mathrm{C}$ & 526 & & 275E Building - Slorm water run-off. & 1.00 & Injection Well & SD2 & $\begin{array}{l}\text { E573,707.84 } \\
\text { N135,613.17 }\end{array}$ & $\begin{array}{l}\text { ADDED 2/95. } 10^{\circ} \text { East of the } \\
\text { northeast comer of } 275 E \text { Building. }\end{array}$ & Yes & Yes \\
\hline 2001: & $\mathrm{D}$ & 318 & & $\begin{array}{l}\text { 275EA Building - Steam condensale; batch } \\
\text { discharged during winter. }\end{array}$ & $<5.00$ & Injection Well & $5 W 20$ & $\begin{array}{l}\text { ES75,082.26 } \\
\text { NI35,839.39 } \\
\end{array}$ & 27SEA west șide & No & Yes \\
\hline 2001: & $\mathrm{D}$ & 497 & & $\begin{array}{l}\text { 275EA luilding - Steam condensate from } \\
\text { steam pipeline. }\end{array}$ & $<0.01$ & Injection Well & $5 W 20$ & $\begin{array}{l}\text { EST5,155.34 } \\
\text { N135,903.89 } \\
\end{array}$ & ADDED 12/94. Southeast of 275EA & No & Yes \\
\hline 2001: & D) & 317 & b & $\begin{array}{l}\text { 276C Building - Sleam condensale; batch } \\
\text { discharged during winter. }\end{array}$ & $<5.00$ & Injection Well & sw20 & $\begin{array}{l}\text { E574,547.21 } \\
N 136,367.39\end{array}$ & $\begin{array}{l}\text { FLIMINA'TED: } 8 / 30 / 95 \text { per cc:mail } \\
\text { from from M. Gunter. } 276 \mathrm{C} \text { east } \\
\text { side }\end{array}$ & No & Yes \\
\hline $2001 \mathrm{~S}$ & $\mathrm{D}$ & 176 & & $\begin{array}{l}\text { 283E Building - IITP-TRP-200-205,208- } \\
305 \text { (located inside this building) discharge } \\
\text { to this injection well. Ileater filter floor. }\end{array}$ & $<5.00$ & Injection Well & $5 W 20$ & $\begin{array}{l}\text { ES73,829.00 } \\
\text { N135,664.32 }\end{array}$ & $\begin{array}{l}\text { CORRECTED S/95. This stream is } \\
\text { to be relocated. }\end{array}$ & No & Yes \\
\hline
\end{tabular}

\begin{tabular}{|c|c|c|c|}
\hline Source Water Kiry & A-Groundwaler & Bu Surface Water & C-Storm Water \\
\hline
\end{tabular}

D-Potable Walet

br Streans discharging to an irjection well wittin a suf face contaminated ase.

d- Disposal site within 300 feet of an active/inactive enb, duch, or trench 
Table 3-1. Miscellaneous Streams Inventory

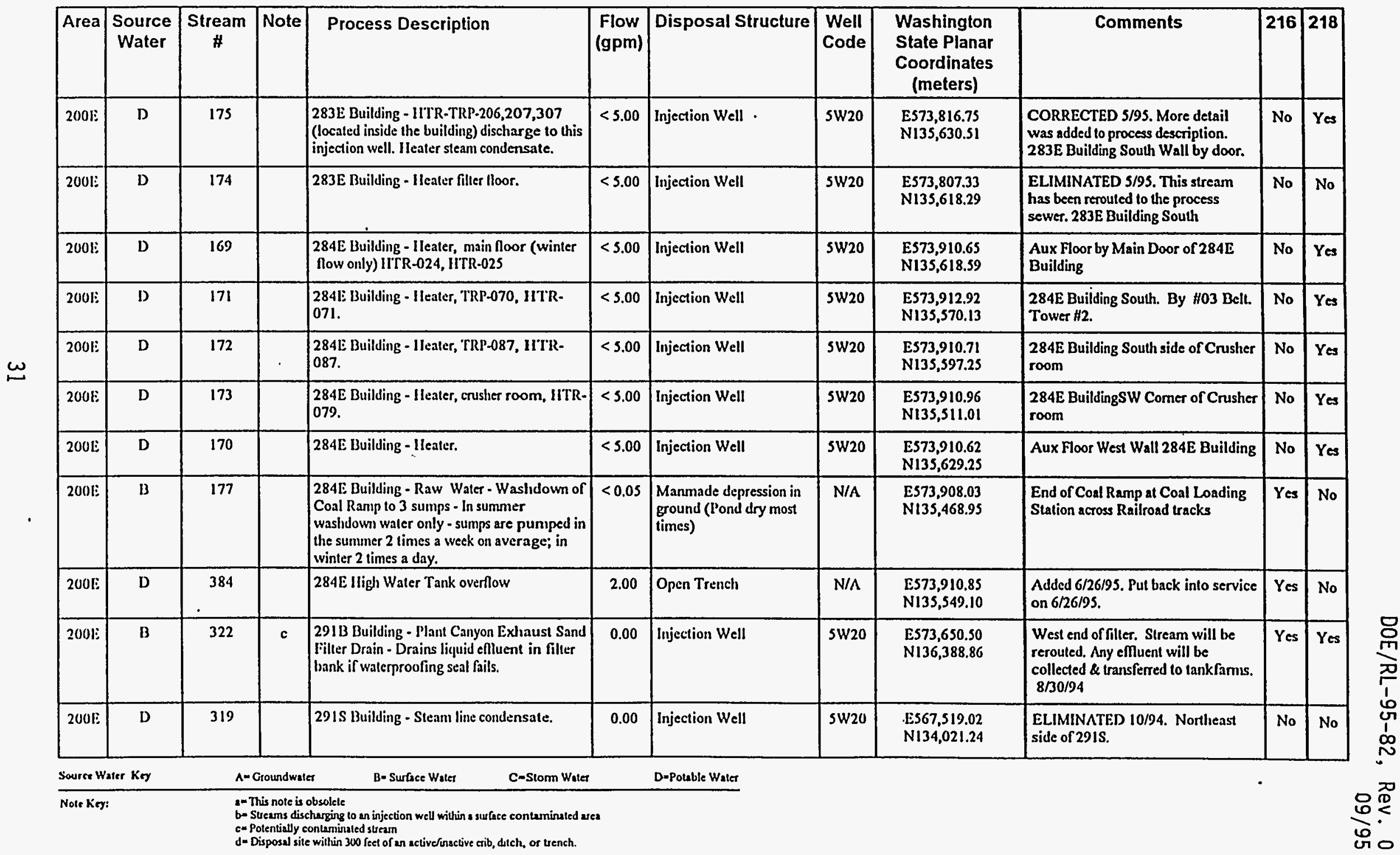


Table 3-1. Miscellaneous Streams Inventory

\begin{tabular}{|c|c|c|c|c|c|c|c|c|c|c|c|}
\hline Area & $\begin{array}{l}\text { Source } \\
\text { Water }\end{array}$ & $\begin{array}{c}\text { Stream } \\
\#\end{array}$ & Note & Process Description & $\begin{array}{c}\text { Flow } \\
\text { (gpm) }\end{array}$ & Disposal Structure & $\begin{array}{c}\text { Well } \\
\text { Code }\end{array}$ & $\begin{array}{l}\text { Washington } \\
\text { State Planar } \\
\text { Coordinates } \\
\text { (meters) }\end{array}$ & Comments & 216 & 218 \\
\hline 2001: & $D$ & 320 & & 291S Building - Steam turbine condensate. & 0.00 & Injection Well & $5 W 20$ & $\begin{array}{l}\text { ES67,511.43 } \\
\text { N134,009.03 }\end{array}$ & $\begin{array}{l}\text { ELIMINATED 10/94. Southwest } \\
\text { side of } 291 \mathrm{~S} \text {. }\end{array}$ & No & No \\
\hline $2001:$ & $\mathrm{D}$ & 8 & d & $\begin{array}{l}29213 \text { Building - B Plant yard steam line, } \\
\text { three inch line to 292-B, steam trap } \\
\text { condensate. }\end{array}$ & 0.01 & Injection Well & SW 20 & $\begin{array}{l}\text { E } 573,556.52 \\
\text { N136,375.54 }\end{array}$ & $\begin{array}{l}\text { "d" note added per weissenfels on } \\
8 / 28 / 95\end{array}$ & No & Yes \\
\hline 2001: & D & 5 & $d$ & $\begin{array}{l}\text { B Plant Yard Stcan Line - Eighlt inch mait, } \\
\text { stean trap condensate. }\end{array}$ & 0.70 & Injection Well & $5 W 20$ & $\begin{array}{l}\text { ES73,411.47 } \\
\text { N136,367.91 }\end{array}$ & $\begin{array}{l}\text { "d" nole added per weissenfels on } \\
8 / 28 / 95\end{array}$ & No & Yes \\
\hline $2001:$ & $\mathfrak{D}$ & 6 & d & $\begin{array}{l}\text { B Plant Yard Steam Line - Eight inch main, } \\
\text { steam trap condensate. }\end{array}$ & 0.30 & Injection Well & $5 W 20$ & $\begin{array}{l}\text { Es73,358.44 } \\
\text { N136,367.76 }\end{array}$ & $\begin{array}{l}\text { "d" note added per weissenfels on } \\
8 / 28 / 95\end{array}$ & No & Yes \\
\hline $2001:$ & D & 7 & & $\begin{array}{l}\text { 13 Plant Yard Steam Line - Eight inch main, } \\
\text { stean trap condensate. }\end{array}$ & 0.30 & Injection Well & $5 W 20$ & $\begin{array}{l}\text { E.573,330.16 } \\
\text { N136,401.42 }\end{array}$ & & No & Yes \\
\hline 2001: & $\mathrm{D}$ & 1 & & $\begin{array}{l}\text { B Plant Yard Steam line - Six inch main, } \\
\text { steam Irap condensate. }\end{array}$ & 0.80 & Injection Well & $5 W 20$ & $\begin{array}{l}E 573,721.72 \\
\text { N136,368.79 }\end{array}$ & . & No & Yes \\
\hline 2000: & D & 4 & d & $\begin{array}{l}\text { B Plant Yard Steam Line - Ten inch main, } \\
\text { steam trap condensate. }\end{array}$ & 0.50 & Injection Well & $5 W 20$ & $\begin{array}{l}E 573,560.20 \\
N 136,368.33\end{array}$ & $\begin{array}{l}\text { "d" noted added per weissenfels } \\
8 / 28 / 95\end{array}$ & No & Yes \\
\hline $2001:$ & D & 3 & & $\begin{array}{l}\text { B Plant Yard Steam Line - Ten inch main, } \\
\text { steam trap condensale. }\end{array}$ & 0.40 & Injection Well & $3 w 20$ & $\begin{array}{l}\text { E573,643.09 } \\
\text { Ni36,368.57 }\end{array}$ & & No & Yes \\
\hline 2001: & D) & 9 & d & $\begin{array}{l}\text { B Plant Yard Steam Line - Three inch main, } \\
\text { steam trap condensale. }\end{array}$ & 0.10 & Injection Well & $5 W 20$ & $\begin{array}{l}\text { ES73,277.50 } \\
N 136,377.49\end{array}$ & $\begin{array}{l}\text { "d" note added per wcissenfels on } \\
8 / 28 / 95\end{array}$ & No & Yes \\
\hline 2001: & D & 2 & & $\begin{array}{l}\text { B Plant Yard Steam Line - Twelve inch } \\
\text { main, steam trap condensate. }\end{array}$ & 0.05 & Injection Well & sw20 & $\begin{array}{l}\text { Es73,714.10 } \\
\text { N136,368.77 }\end{array}$ & & No & Yes \\
\hline $2001:$ & B & 688 & & $\begin{array}{l}\text { ETF Trealment Facility: consinuction } \\
\text { discharge from sump lanks } \# 1 \text { and } \# 2 \text {. }\end{array}$ & 0.00 & to ground & & E0.00 N0.00 & $\begin{array}{l}\text { Discharge to ground at the NE comer } \\
\text { and south end of building. This } \\
\text { discharge will be covered under the } \\
\text { first categorical permit. }\end{array}$ & Yes & No \\
\hline $2001:$ & c & 558 & & $\begin{array}{l}\text { M0400 Building - Storm water drains to } \\
\text { ground will overtlow to sewer. } \\
\text {. }\end{array}$ & $<1.00$ & Injection Well & SD2 & $\begin{array}{l}E 573,463.12 \\
\text { N136,533.15 }\end{array}$ & $\begin{array}{l}\text { ADDED 2/95. } 20 \text { feet south of } \\
\text { southwest comer of M0400 near B- } \\
\text { Plant. }\end{array}$ & Yes & Yes \\
\hline
\end{tabular}

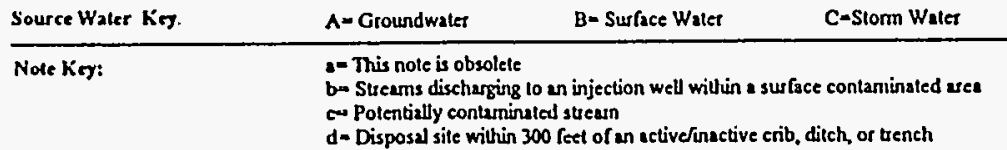

D-Poluble Water 
Table 3-1. Miscellaneous Streams Inventory

\begin{tabular}{|c|c|c|c|c|c|c|c|c|c|c|c|}
\hline Area & $\begin{array}{c}\text { Source } \\
\text { Water } \\
\end{array}$ & $\begin{array}{c}\text { Stream } \\
\#\end{array}$ & Note & Process Description & $\begin{array}{c}\text { Flow } \\
\text { (gpm) }\end{array}$ & Disposal Structure & $\begin{array}{l}\text { Well } \\
\text { Code }\end{array}$ & $\begin{array}{l}\text { Washington } \\
\text { State Planar } \\
\text { Coordinates } \\
\text { (meters) }\end{array}$ & Comments & 216 & 218 \\
\hline 2001: & $\mathrm{D}$ & 533 & & $\begin{array}{l}\text { MO035 Building - Source is water valve on } \\
\text { line providing water to trailer. }\end{array}$ & $<1.00$ & Injection Well & $5 w_{20}$ & $\begin{array}{l}\text { ES75,009.78 } \\
\text { N136,019.07 }\end{array}$ & $\begin{array}{l}\text { ADDED 2/95. } 3 \text { feet south of trailer } \\
\text { towards center near PUREX. }\end{array}$ & Yes & Yes \\
\hline $2001:$ & D & 101 & & $\begin{array}{l}\text { Steam Trap - 2P-Yard-MSS-TRP-048 } \\
\text { (formerly MSD HOI)-Steam Condensalc. }\end{array}$ & $<1.00$ & Injection Well & $5 W 20$ & $\begin{array}{l}\text { ES73,602.64 } \\
\text { N135,686.28 }\end{array}$ & $\begin{array}{l}\text { CORRECTED: 7/6/95 PER } \\
\text { CC:MAIL from M. Gunter. East of } \\
\text { 272E }\end{array}$ & No & Yes \\
\hline 200E: & D & 642 & & $\begin{array}{l}\text { Steain Trap - 2P-Yard-MSS-TRP-050- } \\
\text { Sleam condensate. }\end{array}$ & $<0.01$ & Injection well & $5 W 20$ & $\begin{array}{l}\text { E573,884.00 } \\
N 135,539.00\end{array}$ & $\begin{array}{l}\text { LADDED 6/95. On line } 104 \text { to } \\
\text { 282E. }\end{array}$ & No & Yes \\
\hline 2005 & D & 643 & & $\begin{array}{l}\text { Steam Trap - 2P-Yard-MSS-TRP-056- } \\
\text { Steam condensate. }\end{array}$ & $<0.01$ & Injection well & $5 W 20$ & $\begin{array}{l}\text { ES73,907.00 } \\
\text { N135,557.00 }\end{array}$ & $\begin{array}{l}\text { ADDED 6/95. On line } 103 \text { to coal } \\
\text { ramp }\end{array}$ & No & Yes \\
\hline $2001:$ & D & 644 & & $\begin{array}{l}\text { Stcam 'Trap - 2P'-Yard-MSS-TRP-057- } \\
\text { Steam condensale. }\end{array}$ & $<0.01$ & Injection well & sw20 & $\begin{array}{l}\text { EST3,944.00 } \\
\text { N135,663.00 } \\
\end{array}$ & ADDED 6/95. INACTIVE. & No & $\overline{Y c s}$ \\
\hline 2001: & D & 645 & & $\begin{array}{l}\text { Steam Trap - 2P-Yard-MSS-TRP-058,059- } \\
\text { Steam condensale. }\end{array}$ & $<0.01$ & Injection well & sw20 & $\begin{array}{l}\text { E573,853.00 } \\
\text { N135,627.00 }\end{array}$ & $\begin{array}{l}\text { ADDED 6/95. Line } 101 \text { to 283E. } \\
\text { Both steam traps discharge to the } \\
\text { same injection well }\end{array}$ & No & Yes \\
\hline $200 \mathrm{E}$ & D & 633 & & $\begin{array}{l}\text { Steam Trap-2R-Yard-MSS-TRP-060- } \\
\text { Steam Condensale }\end{array}$ & $<0.01$ & Injection well & $5 w 20$ & $\begin{array}{l}\text { E573,881.72 } \\
\text { N135,610.88 }\end{array}$ & $\begin{array}{l}\text { ADDED 6/95. Located in front of } \\
284 E \text {, between Streams } 86 \text { and } 87 \text {. }\end{array}$ & No & Yes \\
\hline 2000 & D & 634 & & $\begin{array}{l}\text { Steam Trap - 2P-Yard-MSS-TRP-063- } \\
\text { Steam condensate. }\end{array}$ & $<0.01$ & Injection well & $5 W 20$ & $\begin{array}{l}\text { ES73,877.28 } \\
\text { N135,565.16 }\end{array}$ & $\begin{array}{l}\text { ADDED 6/95. Located in a cassion } \\
\text { in the road. Near stream } 87 \text { across } \\
\text { coad ramp by } 284 E \text {. }\end{array}$ & No & Yes \\
\hline 2001: & D & 635 & & $\begin{array}{l}\text { Steam Trap - 2P-Yard-MSS-TRP-064- } \\
\text { Steam condensale. }\end{array}$ & $<0.01$ & Injection well & $5 W 20$ & $\begin{array}{l}\text { ES73,892.73 } \\
\text { N135,489.02 }\end{array}$ & $\begin{array}{l}\text { ADDED 6/95. Located in a pit near } \\
\text { the silo next to the coal ramp. }\end{array}$ & No & Yes \\
\hline 2001: & D & 86 & & Steam Trap $2 \mathrm{P}$ - Yard-MSS-TRP-001. & $<1.00$ & Injection Well & $5 W 20$ & $\begin{array}{l}\text { ES73,885.00 } \\
\text { N135,640.00 } \\
\end{array}$ & West of $284 \mathrm{E}$ & No & Yes \\
\hline 200E & D & 87 & & Steam Trap 2P - Yard-MSS-TRP-002. & $<1.00$ & Injection Well & $5 W 20$ & $\begin{array}{l}\text { E573,864.00 } \\
\text { NI35,596.00 }\end{array}$ & West of $284 \mathrm{E}$ & No & Yes \\
\hline 2001 & $\mathrm{D}$ & 89 & & Steam Trap 2P - Yard-MSS-TRP-003. & $<1.00$ & Injection Well & $5 W 20$ & $\begin{array}{l}\text { ES73,744.00 } \\
\text { N135,562.00 }\end{array}$ & East side of Baltimore Crossover & No & Yes \\
\hline
\end{tabular}

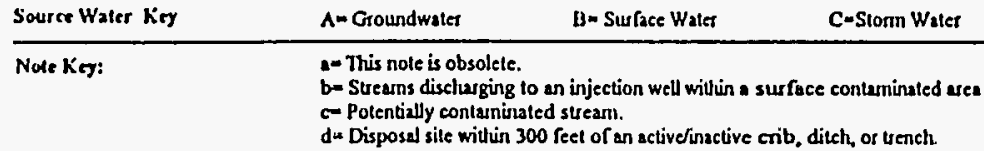

D-Potalle Waler 


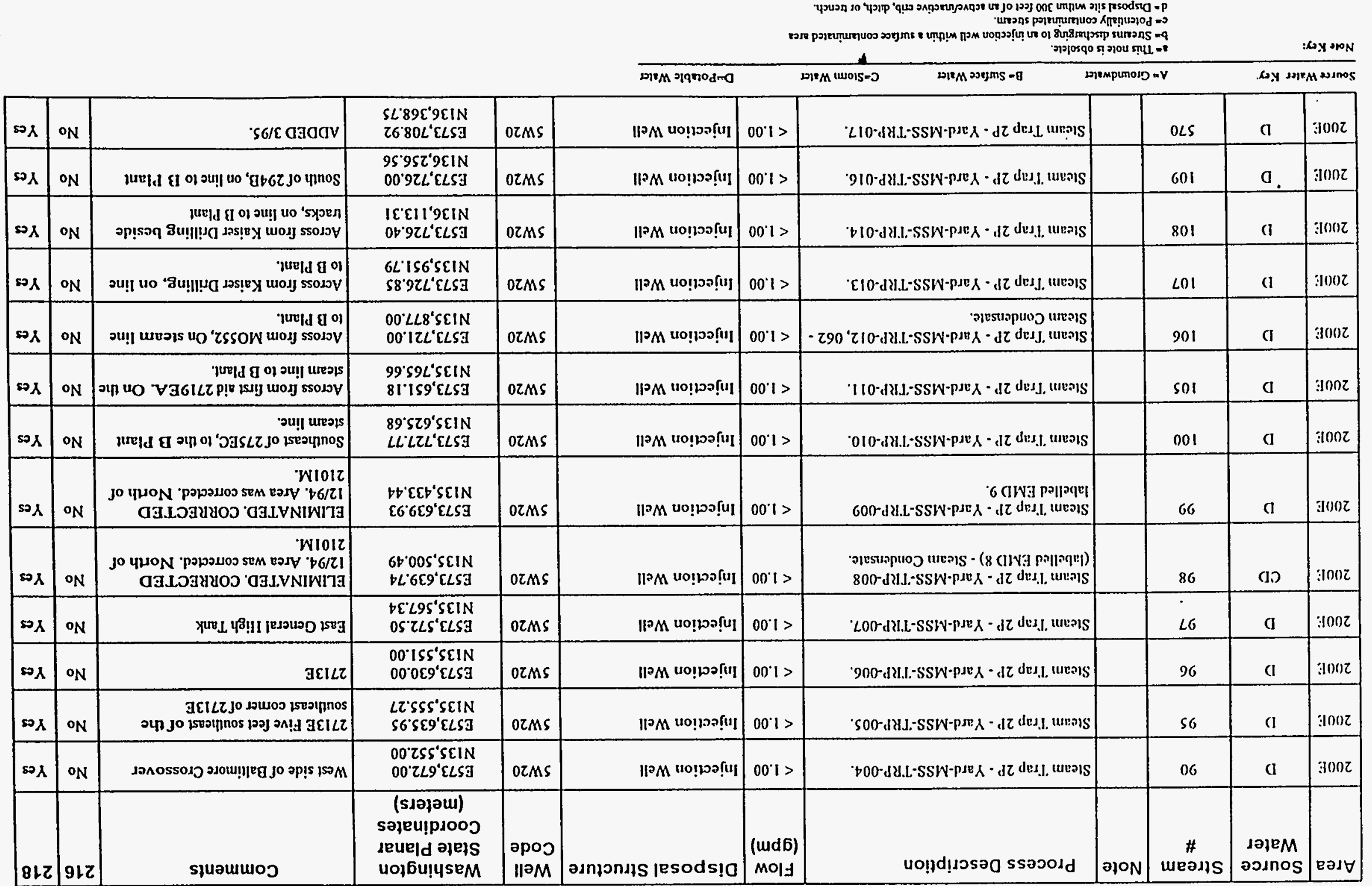

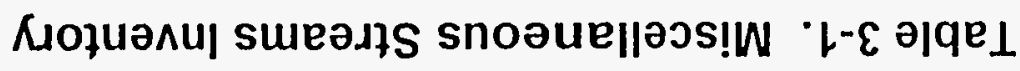


Table 3-1. Miscellaneous Streams Inventory

\begin{tabular}{|c|c|c|c|c|c|c|c|c|c|c|c|}
\hline Area & \begin{tabular}{|l|} 
Source \\
Water
\end{tabular} & $\begin{array}{c}\text { Stream } \\
\#\end{array}$ & Note & Process Description & $\begin{array}{c}\text { Flow } \\
\text { (gpm) }\end{array}$ & Disposal Structure & $\begin{array}{l}\text { Well } \\
\text { Code }\end{array}$ & $\begin{array}{l}\text { Washington } \\
\text { State Planar } \\
\text { Coordinates } \\
\text { (meters) }\end{array}$ & Comments & 216 & 218 \\
\hline $200 \mathrm{E}$ & $\mathrm{D}$ & 110 & & Steam Trap 2P - Yard-MSS-TRP-018. & $<1.00$ & Injection Well & $5 w 20$ & $\begin{array}{l}\text { ES73,753.11 } \\
\text { N } 136,369.40\end{array}$ & $\begin{array}{l}\text { INACTIVE. North of } 294 \mathrm{~B} \text {. On line } \\
\text { to hot semi. }\end{array}$ & No & Yes \\
\hline $200 \mathrm{E}$ & $\mathrm{D}$ & 571 & & Steam Trap 2P - Yard-MSS-TRP-019. & $<1.00$ & Injection Well & $5 W 20$ & $\begin{array}{l}\text { ES73,699.78 } \\
\text { N136,368.73 }\end{array}$ & ADDED 3/95. & No & Yes \\
\hline $200 \mathrm{E}$ & D & 111 & & Stcam Trap 2P - Yard-MSS-TRP-036. & $<1.00$ & Injection Well & sw20 & $\begin{array}{l}\text { ES74,010.92 } \\
\text { N135,727.07 } \\
\end{array}$ & East of 284E toward PUREX & No & Yes \\
\hline $200 \mathrm{E}$ & D & 112 & & Steam Trap 2P - Yard-MSS-TRP-037. & $<1.00$ & Injection Well & $5 W 20$ & $\begin{array}{l}\text { E574,227.31 } \\
\text { N135,727.68 } \\
\end{array}$ & 2nd trap on PUREX line & No & Yes \\
\hline $200 \mathrm{E}$ & D & 113 & & Steam Trap 2P - Yard-MSS-TRP-038. & $<1.00$ & Injection Well & sw20 & $\begin{array}{l}\text { Es74,547.31 } \\
\text { N135,728.59 } \\
\end{array}$ & 3rd trap on PUREX line & No & Yes \\
\hline $200 \mathrm{E}$ & D & 114 & & Steam Trap 2P-Yard-MSS-TRP-039. & $<1.00$ & Injection Well & sw20 & $\begin{array}{l}\text { ES74,736.28 } \\
\text { N135,729.14 } \\
\end{array}$ & 4th trap on PUREX line & No & Yes \\
\hline 200E & D & 115 & & Steam Trap 2P - Yard-MSS-TRP-040. & $<1.00$ & Injection Well & $5 W 20$ & $\begin{array}{l}\text { E575,053.47 } \\
\text { N135,766.32 } \\
\end{array}$ & Caisson Pit from PUREX 10 204-AR. & No & Yes \\
\hline $200 \mathrm{E}$ & D & 116 & & Steam Trap 2P - Yard-MSS-TRP-041. & $<1.00$ & Injection Well & $5 W 20$ & $\begin{array}{l}\text { Es75,056.00 } \\
\text { N135,887.00 } \\
\end{array}$ & West of 275EA & No & Yes \\
\hline $200 \mathrm{E}$ & $\mathrm{D}$ & 117 & & Steam Trap 2P-Yard-MSS-TRP-042. & $<1.00$ & Injection Well & $5 W 20$ & $\begin{array}{l}\text { E575, } 166.00 \\
\text { N135,947.00 } \\
\end{array}$ & North of 27SEA & No & $\overline{\text { Yes }}$ \\
\hline $200 \mathrm{E}$ & D & 572 & & Steam Trap 2P-Yard-MSS-TRP-043. & $<1.00$ & Injection Well & $5 W 20$ & $\begin{array}{l}\text { ES75,181.00 } \\
\text { NI35,986.00 } \\
\end{array}$ & ADDED 3/95. North of 275EA & No & Yes \\
\hline $200 \mathrm{E}$ & D & 573 & & Steam Trap 2P - Yard-MSS-TRP-044. & $<1.00$ & Injection Well & $5 W 20$ & $\begin{array}{l}\text { E575,194.00 } \\
\text { N136,621.00 } \\
\end{array}$ & ADDED 3/95. West of 244AR & No & $\overline{\text { Yes }}$ \\
\hline 200E & D & 91 & & Steam Trap 2P - Yard-MSS-TRP-047. & 0.00 & & & $\begin{array}{l}\text { E573,660.87 } \\
\text { N135,573.68 }\end{array}$ & $\begin{array}{l}\text { ELIMINATED; } 9 / 5 / 95 \text {, stream does } \\
\text { not discharge to an engineered } \\
\text { structure, per cemail from M. } \\
\text { Gunter. South of } 2715 E\end{array}$ & No & No \\
\hline 200E & D & 104 & & Steam Trap 2P - Yard-MSS-TRP-049. & $<1.00$ & Injection Well & $5 W 20$ & $\begin{array}{l}\text { ES73,602.73 } \\
\text { N135,655.81 }\end{array}$ & Northeast of 272E & No & Yes \\
\hline
\end{tabular}


Table 3-1. Miscellaneous Streams Inventory

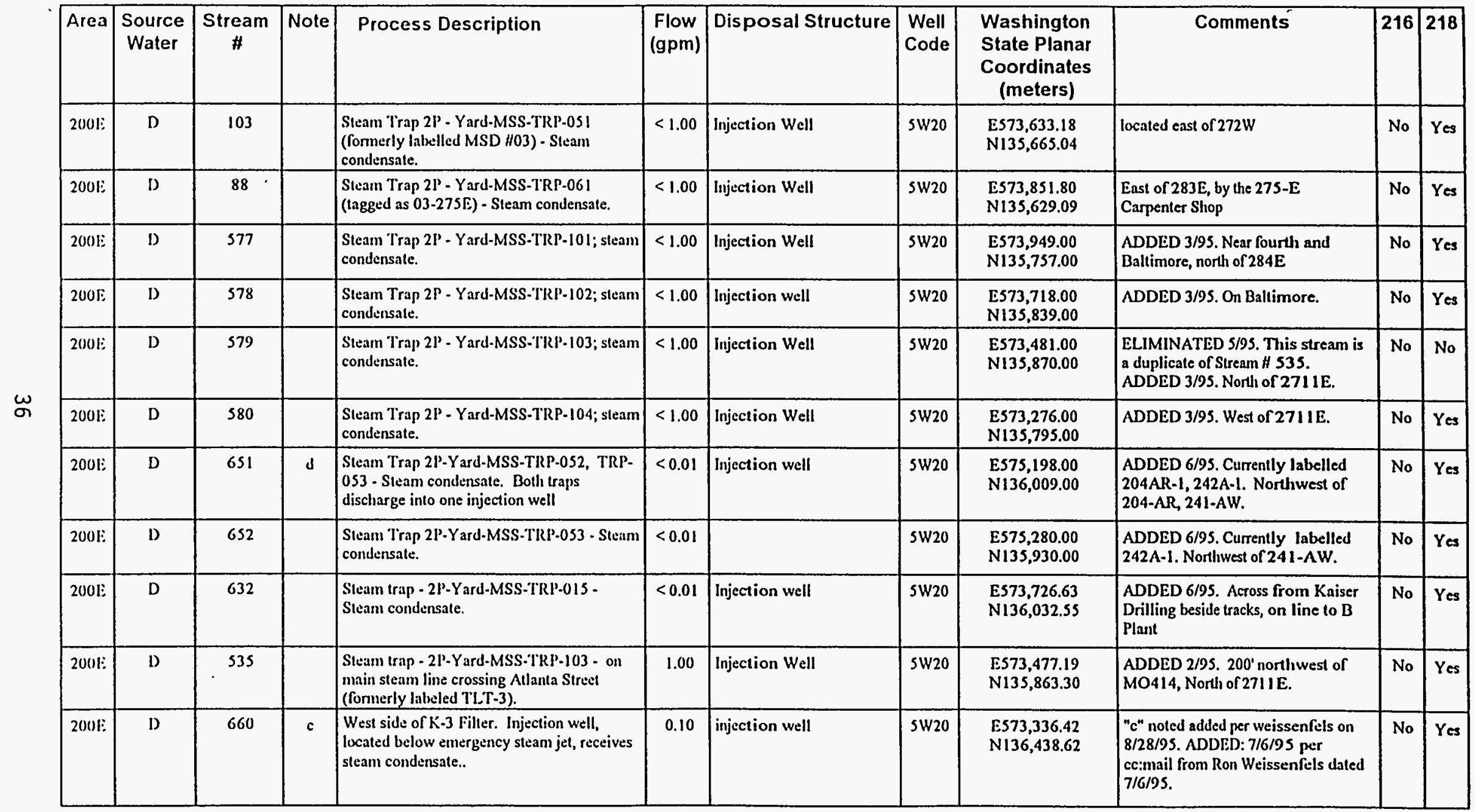

Source Waler Ker A- Groundwater 
Table 3-1. Miscellaneous Streams Inventory

\begin{tabular}{|c|c|c|c|c|c|c|c|c|c|c|c|}
\hline Area & $\begin{array}{l}\text { Source } \\
\text { Water }\end{array}$ & $\begin{array}{c}\text { Stream } \\
\#\end{array}$ & Note & Process Description & \begin{tabular}{|c|} 
Flow \\
(gpm)
\end{tabular} & Disposal Structure & \begin{tabular}{|l|} 
Well \\
Code
\end{tabular} & $\begin{array}{l}\text { Washington } \\
\text { State Planar } \\
\text { Coordinates } \\
\text { (meters) }\end{array}$ & Comments & 216 & 218 \\
\hline $200 \mathrm{w}$ & D & 202 & d & 219S Building - Steam condensate. & $<1.00$ & Injection Well & sw20 & $\begin{array}{l}E 567,473.66 \\
\mathrm{~N} 133,928.20 \\
\end{array}$ & North side of 219S. & No & Yes \\
\hline $200 w$ & $\mathrm{D}$ & 575 & $d$ & 219S Building - Steam condensate. & $<1.00$ & Injection Well & $5 W 20$ & $\begin{array}{l}\text { Es67,466.04 } \\
\text { N133,929.71 } \\
\end{array}$ & $\begin{array}{l}\text { ADDED 3/95. 219S Building, west } \\
\text { of the main door. }\end{array}$ & No & Yes \\
\hline $200 \mathrm{w}$ & $\mathrm{D}$ & 200 & d & 222S Builuing - Steam condensate. & $<1.00$ & Injection Well & $5 \mathrm{~W} 20$ & $\begin{array}{l}E 567,424.94 \\
N 133,906.14\end{array}$ & Localed near Door 15 North. & No & Yes \\
\hline $200 w$ & c & 206 & & $\begin{array}{l}\text { 222S Building - Calch Basin (storm drain) } \\
\text { H03. }\end{array}$ & $<0.10$ & Injection Well & SD2 & $\begin{array}{l}\text { E567,304.S4 } \\
N 133,814.86\end{array}$ & \begin{tabular}{|l|} 
CHANGED 6/95. Upon review of \\
maps and current drawings this catch \\
basin was found to not be a "d." In \\
addition, the well code was changed \\
to a SD2 to reflect the BMP \\
downgrade. 222S Lab parking area.
\end{tabular} & Yes & Yes \\
\hline $200 \mathrm{~W}$ & c & 207 & & $\begin{array}{l}\text { 222S Building - Catch Basin (storm drain) } \\
\text { 104. }\end{array}$ & $<0.10$ & Injection Well & SD2 & $\begin{array}{l}\text { ES567,256.62 } \\
\text { N133,859.24 }\end{array}$ & $\begin{array}{l}\text { CIIANGED 6/9S. Upon review of } \\
\text { maps and currest drawings this catch } \\
\text { basin was found to not be a "d." In } \\
\text { addition, the well code was changed } \\
\text { to a SD2 to reflet the BMP } \\
\text { downgrade. 222S Lab parking area. }\end{array}$ & Yes & Yes \\
\hline $200 \mathrm{~W}$ & C & 208 & d & $\begin{array}{l}\text { 222S l3uilling - Catch Basin (slonn drain) } \\
\text { Hos. }\end{array}$ & $<0.10$ & Injection Well & SD4 & $\begin{array}{l}\text { E567,354.19 } \\
\text { N133,821.97 } \\
\end{array}$ & 222S Lab parking area. & Yes & Yes \\
\hline $200 \mathrm{w}$ & c & 209 & d & $\begin{array}{l}\text { 222S Building - Calch Basin (stonn drain) } \\
\text { 106. }\end{array}$ & $<0.10$ & Injection Well & 3D4 & $\begin{array}{l}\text { ES67,353.99 } \\
N 133,920.07 \\
\end{array}$ & 222S Lab parking area. & Yes & Yes \\
\hline $2001 \mathrm{~W}$ & $\mathrm{c}$ & 210 & $d$ & $\begin{array}{l}\text { 222S Building - Catch Basin (storn drain) } \\
\text { H07. }\end{array}$ & $<0.10$ & Injection Well & SD4 & $\begin{array}{l}\text { ES67,360.91 } \\
\text { N133,814.97 } \\
\end{array}$ & 222S Lab parking area. & Yes & Yes \\
\hline $200 \mathrm{w}$ & $\mathrm{c}$ & 211 & $d$ & $\begin{array}{l}\text { 222S Building - Calch Basin (stonn drain) } \\
\text { 1108. }\end{array}$ & $<0.10$ & Injection Well & SD4 & $\begin{array}{l}\text { ES67,360.69 } \\
\text { N133,917.04 } \\
\end{array}$ & 222S Lab parking area. & Yes & Yes \\
\hline $200 w$ & C & 212 & $d$ & $\begin{array}{l}\text { 222S Building - Calch Basin (stom drain) } \\
\text { "110. }\end{array}$ & $<0.10$ & Injection Well & SD4 & $\begin{array}{l}\text { ES67,433.98 } \\
\text { N133,815.01 } \\
\end{array}$ & $222 S$ Lab parking area. & Yes & Yes \\
\hline $200 \mathrm{w}$ & c & 213 & d & $\begin{array}{l}\text { 222S Building - Catch Basin (stonn drain) } \\
\text { H11. }\end{array}$ & $<0.10$ & Injection Well & 5D4 & $\begin{array}{l}\text { Es67,389.85 } \\
\text { N133,920.39 }\end{array}$ & $222 S$ Lab parking area. & Yes & Yes \\
\hline
\end{tabular}


Table 3-1. Miscellaneous Streams Inventory

\begin{tabular}{|c|c|c|c|c|c|c|c|c|c|c|c|}
\hline Area & $\begin{array}{l}\text { Source } \\
\text { Water }\end{array}$ & $\begin{array}{c}\text { Stream } \\
\#\end{array}$ & Note & Process Description & \begin{tabular}{|c|} 
Flow \\
(gpm)
\end{tabular} & Disposal Structure & $\begin{array}{l}\text { Well } \\
\text { Code }\end{array}$ & $\begin{array}{c}\text { Washington } \\
\text { State Planar } \\
\text { Coordinates } \\
\text { (meters) }\end{array}$ & Comments & 216 & 218 \\
\hline 2000 & D & 576 & d & 222S Building - Steam condensate. & $<1.00$ & Injection Well & $5 W_{20}$ & $\begin{array}{l}E 567,418.63 \\
N 133,929.60\end{array}$ & $\begin{array}{l}\text { ADDED 3/95. To be constructed in } \\
\text { 5/95. Located north of door } 15 \\
\text { (222S), near the roadway. }\end{array}$ & No & Yes \\
\hline $2000 \mathrm{~W}$ & $\bar{c}$ & 584 & J & $\begin{array}{l}2225 \text { Building - drain line collects overflow } \\
\text { storm water from catch basin } 166 \text { and } H 8 . \\
\text { Drain line is a } 100^{\circ}, 15^{\prime \prime} \text { dia. perforated } \\
\text { comugated metal pipe. }\end{array}$ & $<0.20$ & Drain Line & SD4 & $\begin{array}{l}\text { E566,951.87 } \\
\text { N133,893.65 }\end{array}$ & $\begin{array}{l}\text { ADDED 4/95. The drain line begins } \\
\text { 20' west of catch basin } H 6 \text {, } \\
\text { extending } 100^{\prime} \text { westward. }\end{array}$ & Yes & No \\
\hline $200 w$ & $\mathrm{D}$ & 201 & d & 222SC Building - Steam condensale. & $<1.00$ & Injection Well & sw20 & $\begin{array}{l}\text { Es67,436.53 } \\
\text { N133,926.59 } \\
\end{array}$ & North of building. & No & Yes \\
\hline $200 \mathrm{~W}$ & D & 271 & & 222T Building - Steam condensate. & $<1.00$ & Injection Well & sw20 & $\begin{array}{l}\text { E567,629.50 } \\
\text { N136,816.09 } \\
\end{array}$ & & No & Yes \\
\hline $200 \mathrm{w}$ & D & 272 & & 222T Building - Steam condensale. & $<1.00$ & Injection Well & sw20 & $\begin{array}{l}\text { E567,621.64 } \\
\text { N136,804.95 } \\
\end{array}$ & & No & Yes \\
\hline $200 w$ & D & 273 & & $222 \mathrm{~T}$ Building - Stean condensale. & $<1.00$ & Injection Well & sw20 & $\begin{array}{l}\text { E567,613.77 } \\
\text { N136,789.88 } \\
\end{array}$ & & No & Yes \\
\hline $200 \mathrm{w}$ & D & 274 & & 222 $\mathrm{r}$ Building - Stean condensate. & $<1.00$ & Injection Well & $5 W_{20}$ & $\begin{array}{l}\text { E567,615.74 } \\
\text { N136,797.09 } \\
\end{array}$ & . & No & Yes \\
\hline $200 \mathrm{w}$ & D & 275 & & 222T Building - Steam condensate. & $<1.00$ & Injection Well & $5 W_{20}$ & $\begin{array}{l}\text { ES67,626.23 } \\
\text { N136,811.51 } \\
\end{array}$ & & No & Yes \\
\hline $200 \mathrm{w}$ & $\mathrm{CD}$ & 393 & & $\begin{array}{l}\text { 222U Building - Steam condensate and } \\
\text { storm water. }\end{array}$ & $<1.00$ & Injection Well & $5 W 20$. & $\begin{array}{l}\text { ES67,661.04 } \\
\text { N135,103.63 }\end{array}$ & $\begin{array}{l}\text { CORRECTED 2/95. Steam is } \\
\text { INACTIVE. 222U back side eastem } \\
\text { most comer. }\end{array}$ & Yes & Yes \\
\hline $200 \mathrm{w}$ & $C D$ & 394 & & $\begin{array}{l}222 U \text { Building · Steam condensale and } \\
\text { storm water. }\end{array}$ & $<1.00$ & Injection Well & $5 W 20$, & $\begin{array}{l}\text { E567,603.14 } \\
\text { N135,115.94 }\end{array}$ & $\begin{array}{l}\text { CORRECTED 2/95. Steam is } \\
\text { INACTIVE. } 222 U \text { back side } \\
\text { western most comer. }\end{array}$ & Yes & \begin{tabular}{|l|} 
Yes \\
\end{tabular} \\
\hline $200 w$ & c & 521 & & 222U Building - Storm water run-ofr. & $<0.50$ & Injection Well & SD2 & $\begin{array}{l}\text { ES67,612.76 } \\
\text { N } 135,127,60\end{array}$ & $\begin{array}{l}\text { ADDED 2/95. Backside of 222U } \\
\text { center of building. }\end{array}$ & Yes & Yes \\
\hline $200 \mathrm{w}$ & c & 687 & & 222. U Building - storm water run-off:' & $<0.50$ & Injection well & SD2 & $\begin{array}{l}E 567,624.13 \\
N 135,141.59\end{array}$ & $\begin{array}{l}\text { ADDED: 9/5/95 per ccrnail from } M \text {. } \\
\text { Gunter }\end{array}$ & Yes & Yes \\
\hline \multicolumn{12}{|c|}{$A=$ Groundwater } \\
\hline \multicolumn{12}{|c|}{ 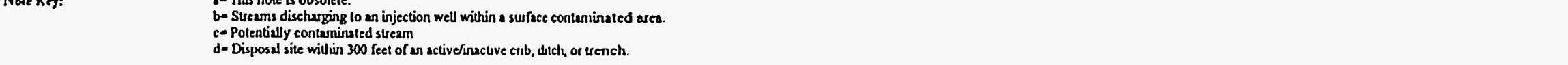 } \\
\hline
\end{tabular}


Table 3-1. Miscellaneous Streams Inventory

\begin{tabular}{|c|c|c|c|c|c|c|c|c|c|c|c|}
\hline Area & $\begin{array}{l}\text { Source } \\
\text { Water }\end{array}$ & $\begin{array}{c}\text { Stream } \\
\quad \#\end{array}$ & Note & Process Description & $\begin{array}{l}\text { Flow } \\
\text { (gpm) }\end{array}$ & Disposal Structure & $\begin{array}{l}\text { Well } \\
\text { Code }\end{array}$ & $\begin{array}{l}\text { Washington } \\
\text { State Planar } \\
\text { Coordinates } \\
\text { (meters) }\end{array}$ & Comments & 216 & 218 \\
\hline $200 \mathrm{~W}$ & $\mathrm{c}$ & 685 & & $222 U$ Building - strom water run-off. & $<0.50$ & Injection well & SD2 & $\begin{array}{l}E 567,607.37 \\
\text { N135,121.04 }\end{array}$ & $\begin{array}{l}\text { ADDED: } 9 / 5 / 95 \text { per comail from } M \text {. } \\
\text { Gunter }\end{array}$ & Yes & Yes \\
\hline $200 \mathrm{~W}$ & D) & 677 & & $\begin{array}{l}224 \mathrm{~T} \text { Building. Steam condensite and heal } \\
\text { pump condensale discharge }\end{array}$ & $<0.01$ & Injection Well & $5 W 20$ & $\begin{array}{r}\text { Es67,548.00 } \\
\text { N136,721.00 } \\
\end{array}$ & ADDED: $8 / 30 / 95$ & Yes & Yes \\
\hline $200 \mathrm{~W}$ & $D$ & ss & & $\begin{array}{l}224 \text { U Building - Steam condensate } \\
\text { discharge. }\end{array}$ & 0.10 & lnjection Well & $5 w 20$ & $\begin{array}{l}\text { E567,524.16 } \\
\text { N134,999.67 }\end{array}$ & $\begin{array}{l}\text { INACTIVE. CORRECTED 10/94. } \\
\text { 224U Building southeast side. Does } \\
\text { not discharge to a surface } \\
\text { contaminated area as stated in } \\
\text { original inventory. }\end{array}$ & No & Yes \\
\hline $200 \mathrm{~W}$ & D & 54 & & $\begin{array}{l}224 U \text { Building - Steam condensate, } \\
\text { northwest corner. }\end{array}$ & 0.50 & Injection Well & $5 W 20$ & $\begin{array}{l}E 567,539.33 \\
\text { N135,024.09 } \\
\end{array}$ & INACTIVE S/95. & No & Yes \\
\hline $200 \mathrm{~W}$ & D & 52 & & $\begin{array}{l}224 \text { U Building - Steam condensate, } \\
\text { southwest side. }\end{array}$ & 0.50 & Injection Well & $5 W 20$ & $\begin{array}{l}\text { E567,539.37 } \\
\text { N135,007.33 } \\
\end{array}$ & INACTIVE S/OS. & No & Yes \\
\hline $200 \mathrm{~W}$ & $\mathrm{D}$ & 259 & & 2312 Building - Main steam line trap $/ 102$. & 0.05 & Injection Well & $5 W 20$ & $\begin{array}{l}\text { Es66,448.25 } \\
\text { N135,885.04 } \\
\end{array}$ & & No & Yes \\
\hline $200 \mathrm{~W}$ & D & 260 & d & $\begin{array}{l}2312 \text { Building - Stack demister condensale } \\
\text { drain. }\end{array}$ & 0.02 & Injection Well & sw20 & $\begin{array}{l}\text { E. } 566,461.35 \\
\text { N135,913.58 }\end{array}$ & & Yes & Yes \\
\hline $200 \mathrm{~W}$ & c & 255 & d & $\begin{array}{l}2312 \text { Building - Air intake corridor storn } \\
\text { drains. }\end{array}$ & $<0.01$ & Injection Well & SD4 & $\begin{array}{l}\text { ES66,453.43 } \\
\text { N135,875.93 }\end{array}$ & & Yes & Yes \\
\hline $200 \mathrm{~W}$ & c & 256 & $d$ & $\begin{array}{l}2312 \text { Building - Air intake corridor storm } \\
\text { drains. }\end{array}$ & $<0.01$ & Injection Well & SD4 & $\begin{array}{l}\text { ES } 566,453.43 \\
N 135,876.08 \\
\end{array}$ & & Yes & Yes \\
\hline $200 \mathrm{~W}$ & $\mathrm{c}$ & 257 & $d$ & $\begin{array}{l}2312 \text { Building - Air intake corridor stom } \\
\text { drains. }\end{array}$ & $<0.01$ & Injection Well & 5D4 & $\begin{array}{l}\text { ES } 66,453.43 \\
\text { N135,876.23 } \\
\end{array}$ & & Yes & Yes \\
\hline $200 \mathrm{~W}$ & D & 258 & d & 2312 Building - Main steam line trap 1101. & 0.05 & Injection Well & $5 W 20$ & $\begin{array}{l}\text { ES66,448.25 } \\
\text { N135,889.99 } \\
\end{array}$ & & No & Yes \\
\hline $2010 \mathrm{~W}$ & CD & 508 & & $\begin{array}{l}\text { 2312. Building - Steam condensate and } \\
\text { potentially receiving stom water from } \\
\text { overhead pipes. }\end{array}$ & $<5.00$ & Injection Well & SD4, & $\begin{array}{l}\text { E566,483.61 } \\
\text { NI35,941.29 }\end{array}$ & $\begin{array}{l}\text { ADDED 12/94. Approximately } 60^{\circ} \\
\text { north of norlienst comer of } 2312 \text {, } \\
\text { near slied. }\end{array}$ & Yes & Yes \\
\hline
\end{tabular}

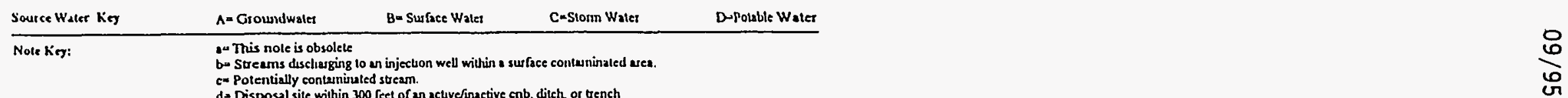


Table 3-1. Miscellaneous Streams Inventory

\begin{tabular}{|c|c|c|c|c|c|c|c|c|c|c|c|}
\hline Area & \begin{tabular}{|c|}
$\begin{array}{c}\text { Source } \\
\text { Water }\end{array}$ \\
\end{tabular} & $\begin{array}{c}\text { Stream } \\
\#\end{array}$ & Note & Process Description & $\begin{array}{l}\text { Flow } \\
\text { (gpm) }\end{array}$ & Disposal Structure & \begin{tabular}{l|} 
Well \\
Code
\end{tabular} & $\begin{array}{c}\text { Washington } \\
\text { State Planar } \\
\text { Coordinates } \\
\text { (meters) }\end{array}$ & Comments & 216 & 218 \\
\hline $200 \mathrm{w}$ & Cl & 509 & & $\begin{array}{l}2312 \text { Building - Slean condensate and } \\
\text { potentially receiving storm water from } \\
\text { overhead pipes. }\end{array}$ & $<5.00$ & Injection Well & SD4, & $\begin{array}{l}\text { ES66,496.79 } \\
\text { N135,920.85 }\end{array}$ & $\begin{array}{l}\text { ADDED 12/94. Within the } \\
\text { northwest inverted comer of } 2312 \\
\text { building }\end{array}$ & Yes & Yes \\
\hline $200 \mathrm{w}$ & CD & 510 & c & $\begin{array}{l}2312 \text { Building - Stcam condunsale and storm } \\
\text { water, potentially contaminated with } \\
\text { hydrocarbons }\end{array}$ & $<5.00$ & Injection Well & SD4, & $\begin{array}{l}\text { ES66,466.16 } \\
\text { NI35,867.13 }\end{array}$ & $\begin{array}{l}\text { ADDED 12/94. Approximately 10 a } \\
\text { east of the southwest comer of the } \\
\text { west wing }\end{array}$ & Yes & Yes \\
\hline $200 \mathrm{w}$ & c & 565 & & $231 Z$ Building - Storm water. & $<0.01$ & Injection Well & SD2 & $\begin{array}{l}\text { E566,453.43 } \\
\text { N135,908.83 }\end{array}$ & $\begin{array}{l}\text { ADDED 2/95. Located in space } \\
\text { between } 2 \text { walls on West side of } \\
\text { 231Z. Northern most of wells in that } \\
\text { area. }\end{array}$ & Yes & $\overline{\text { Yes }}$ \\
\hline $200 \mathrm{~W}$ & c & 566 & & 2312 Building - Stomm water. & $<0.01$ & Injection Well & SD2 & $\begin{array}{l}\text { ES66,453.43 } \\
\text { N135,901.70 }\end{array}$ & $\begin{array}{l}\text { ADDED 2/95. Located in space } \\
\text { belween } 2 \text { walls on West side of } \\
2312 \text {. Second northem most well. }\end{array}$ & Yes & Yes \\
\hline $200 \mathrm{w}$ & c & 567 & & 2312 Building - Storn water. & $<0.01$ & Injection Well & SD2 & $\begin{array}{l}\text { ES66,453.43 } \\
\text { N135,894.05 }\end{array}$ & $\begin{array}{l}\text { ADDED 2/95. Located in space } \\
\text { between } 2 \text { walls on West side of } \\
2312 \text {. Second southern most well. }\end{array}$ & Yes & Yes \\
\hline $200 \mathrm{w}$ & c & 568 & & $231 \mathrm{Z}$ Building - Storn water. & $<0.01$ & Injection Well & SD2 & $\begin{array}{l}\text { ES66,453.43 } \\
N 135,887.39\end{array}$ & $\begin{array}{l}\text { ADDED 2/95. Located in space } \\
\text { between } 2 \text { walls on West side of } \\
\text { 231Z. Soulhem most well. }\end{array}$ & Yes & Yes \\
\hline $200 \mathrm{w}$ & c & 569 & & 231Z Building - Storm water. & $<0.01$ & Injection Well & SD2 & $\begin{array}{l}\text { ES66,509.96 } \\
\text { N135,864.06 }\end{array}$ & $\begin{array}{l}\text { ADDED 2/95. Located } \sim 10 \text { feet } \\
\text { East of front door of } 231 Z \text {. }\end{array}$ & Yes & Yes \\
\hline $200 \mathrm{w}$ & CD & 511 & c & $\begin{array}{l}2312 \text { Build ding - Water condensate and oil } \\
\text { hlowdown from building air compressors, } \\
\text { and storn water. Potemtially contaminated } \\
\text { wilh hydrocarbons. } \\
\end{array}$ & $<5.00$ & Injection'Well & 5D4, & $\begin{array}{l}\text { E566,466.11 } \\
\text { NI35,867.46 }\end{array}$ & $\begin{array}{l}\text { ADDED 12/94. Approximately 12 } \\
\text { east of the soulhwest comer of the } \\
\text { west wing }\end{array}$ & Yes & Yes \\
\hline $200 \mathrm{w}$ & D & 245 & & $\begin{array}{l}232 Z \text { Building - Clange room water healer } \\
\text { overtlow. }\end{array}$ & 0.00 & Injection Well & $5 W_{20}$ & $\begin{array}{l}E 566,440.84 \\
\text { N135,576.08 } \\
\end{array}$ & ELIMINATED 6/95. & Yes & Yes \\
\hline $200 \mathrm{w}$ & D & 248 & & 2345Z Building - Main steam line trap H01. & 0.05 & Injection Well & $5 W 20$ & $\begin{array}{l}\text { E566,45S.54 } \\
\text { N135,778.67 }\end{array}$ & & No & Yes \\
\hline
\end{tabular}

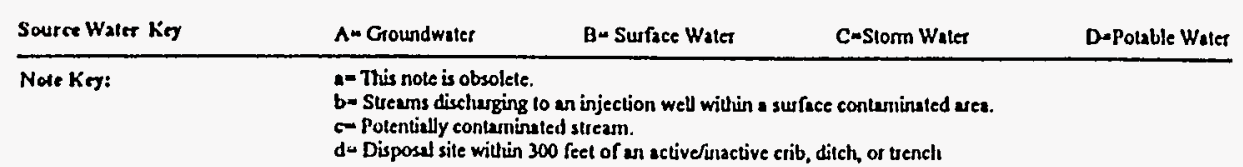


Table 3-1. Miscellaneous Streams Inventory

\begin{tabular}{|c|c|c|c|c|c|c|c|c|c|c|c|}
\hline Area & $\begin{array}{l}\text { Source } \\
\text { Water }\end{array}$ & $\begin{array}{c}\text { Stream } \\
\#\end{array}$ & Note & Process Description & $\begin{array}{c}\text { Flow } \\
\text { (gpm) }\end{array}$ & Disposal Structure & $\begin{array}{l}\text { Well } \\
\text { Code }\end{array}$ & $\begin{array}{c}\text { Washington } \\
\text { State Planar } \\
\text { Coordinates } \\
\text { (meters) }\end{array}$ & Comments & 216 & 218 \\
\hline $200 \mathrm{~W}$ & $\mathrm{D}$ & 249 & & $2345 Z$ Building - Main steam line trap 1102 & 0.05 & Injection Well & $5 w 20$ & $\begin{array}{l}E 566.455 .82 \\
\text { N135,716.25 }\end{array}$ & & No & Yes \\
\hline $200 \mathrm{~W}$ & D & 250 & & 2345Z Building - Main steam line trap $\# 03$. & 0.05 & Injection Well & $5 w 20$ & $\begin{array}{l}E .566,455.89 \\
\text { N135,685.77 } \\
\end{array}$ & - & No & Yes \\
\hline $2000 \mathrm{~W}$ & D & 254 & $d$ & $\begin{array}{l}2345 Z \text { Building - 'PF' Complex main steam } \\
\text { tine trap } \$ 01 .\end{array}$ & 0.05 & Injection Well & $5 W 20$ & $\begin{array}{r}E 566,559.29 \\
\text { N135,795.75 } \\
\end{array}$ & & No & Yes \\
\hline $200 \mathrm{~W}$ & D & 587 & d & $\begin{array}{l}2345 Z \text { Building - PJPP Complex main steam } \\
\text { line trap } \# 2 \text {. }\end{array}$ & 0.05 & Injection Well & $s W 20$ & $\begin{array}{l}\text { E566,534.67 } \\
\text { N135,804.63 } \\
\end{array}$ & ELIMINATED 6/95. ADDED 3/95. & No & Yes \\
\hline $200 w$ & $\mathbf{D}$ & 247 & & $\begin{array}{l}\text { 2345Z Building - Plustonium process support } \\
\text { steam trap. }\end{array}$ & 0.01 & Injection Well & $5 W 20$ & $\begin{array}{l}\text { ES66, } 455.92 \\
\text { N135,676.63 } \\
\end{array}$ & & No & Yes \\
\hline $200 \mathrm{w}$ & $\mathrm{C}$ & 228 & & $\begin{array}{l}\text { 2345Z Building - Storn drain in stairwell to } \\
\text { pipe tunnel H01. }\end{array}$ & $<0.01$ & Injection Well & SD2 & $\begin{array}{l}\text { ES66,545.82 } \\
\text { N135,644.95 }\end{array}$ & & Yes & Yes \\
\hline $200 \mathrm{~W}$ & C & 231 & & $\begin{array}{l}2345 Z \text { Building - Stom drain in stainvell to } \\
\text { pipe tunnel } \$ 06 .\end{array}$ & $<0.01$ & Injection Well & SD2 & $\begin{array}{l}E 566,545.89 \\
\text { N135,664.67 } \\
\end{array}$ & & Yes & Yes \\
\hline $200 \mathrm{~W}$ & c & 229 & & $\begin{array}{l}2345 Z \text { Building - Storm drain in stainwell to } \\
\text { pipe tunnel } 1104 \text {. }\end{array}$ & $<0.01$ & Injection Well & SD2 & $\begin{array}{l}\text { ES66,413.25 } \\
\text { N135,633.92 } \\
\end{array}$ & & Yes & Yes \\
\hline $200 \mathrm{~W}$ & c & 230 & & $\begin{array}{l}23452 \text { Building - Storm drain in stairwell to } \\
\text { pipe tunnel th05. }\end{array}$ & $<0.01$ & Injection Well & SD2 & $\begin{array}{l}\text { E566,410.18 } \\
\text { N135,676.51 } \\
\end{array}$ & & $Y e s$ & Yes \\
\hline $200 \mathrm{~W}$ & D) & 246 & & $\begin{array}{l}\text { 2345Z Building - Ventilation condensate } \\
\text { drain from duct level. }\end{array}$ & 0.01 & Injection Well & $.5 W 20$ & $\begin{array}{l}\text { ES66,414.75 } \\
\text { N135,676.52 } \\
\end{array}$ & & No & Yes \\
\hline $2000 \mathrm{~W}$ & D & 225 & & $\begin{array}{l}\text { 234SZC Building - IIVAC condensate } \\
\text { drains from roof. }\end{array}$ & 0.20 & Injection Well & $3 W 20$ & $\begin{array}{l}\text { Es66,562.71 } \\
\text { N135,644.90 }\end{array}$ & $\begin{array}{l}\text { CORRECTED 2/95. Comment and } \\
\text { contact added. Streams } 225 \text { and } 226 \\
\text { are not duplicate. }\end{array}$ & No & Yes \\
\hline $200 \mathrm{~W}$ & D) & 226 & & $\begin{array}{l}\text { 2345ZC Building - IIVAC condensale } \\
\text { drains from roof. }\end{array}$ & 0.20 & Injection Well & $5 W 20$ & $\begin{array}{l}\text { ES66,562.71 } \\
\text { N135,644.90 }\end{array}$ & $\begin{array}{l}\text { CORRECTED 2/95. Comment and } \\
\text { contact added. Streams } 225 \text { and } 226 \\
\text { are not duplicate. }\end{array}$ & No & Yes \\
\hline $200 \mathrm{~W}$ & D & 554 & J & $\begin{array}{l}\text { 24!-SX Tank Fanm - Steam discliarge to a } \\
\text { caisson }\end{array}$ & 0.00 & Injection Well & $5 W 20$ & $\begin{array}{l}E 566,941.15 \\
\text { N } 134,376.48\end{array}$ & $\begin{array}{l}\text { ELIMINATED S/9S. ADDEI) 2/95. } \\
\text { Steam supply was blanked at valve } \\
\text { MSS-V-17. Inside the fence. }\end{array}$ & No & No \\
\hline
\end{tabular}


Table 3-1. Miscellaneous Streams Inventory

\begin{tabular}{|c|c|c|c|c|c|c|c|c|c|c|c|}
\hline Area & $\begin{array}{l}\text { Source } \\
\text { Water }\end{array}$ & $\begin{array}{c}\text { Stream } \\
\#\end{array}$ & Note & Process Description & $\begin{array}{c}\text { Flow } \\
\text { (gpm) }\end{array}$ & Disposal Structure & $\begin{array}{l}\text { Well } \\
\text { Code }\end{array}$ & $\begin{array}{l}\text { Washington } \\
\text { State Planar } \\
\text { Coordinates } \\
\text { (meters) }\end{array}$ & Comments & 216 & 218 \\
\hline $200 \mathrm{w}$ & D & 555 & d & $\begin{array}{l}\text { 241-SX Tank Fanm - Sleam discharge to a } \\
\text { caissoni. }\end{array}$ & 0.00 & Injection Well & sw20 & $\begin{array}{l}\text { ES66,895.12 } \\
\text { N134,376.74 }\end{array}$ & $\begin{array}{l}\text { ELIMINATED 5/95. ADDED 2/95. } \\
\text { Steam supply was blanked at valve. } \\
\text { MSS-V-17. Inside the fence. }\end{array}$ & No & No \\
\hline $200 w$ & D & 556 & d & $\begin{array}{l}\text { 241-SX Tank Fami - Sleam discharge to a } \\
\text { caisson. }\end{array}$ & 0.00 & Injection Well & $5 w 20$ & $\begin{array}{l}E 566,861.30 \\
\text { N134,376.06 }\end{array}$ & $\begin{array}{l}\text { ELIMINATED S/95. ADDED 2/95. } \\
\text { Steam supply blanked at valve MSS- } \\
\text { V-17. Inside the fence. }\end{array}$ & No & No \\
\hline $200 \mathrm{~W}$ & $\mathrm{D}$ & 557 & d & $\begin{array}{l}241-S X \text { Tank IFarm - Sleam discharge to a } \\
\text { caisson. }\end{array}$ & 0.00 & Injection Well & $5 W 20$ & $\begin{array}{l}\text { E566,861.38 } \\
\text { N134,337.68 }\end{array}$ & $\begin{array}{l}\text { ELIMINATED S/95. ADDED 2/95. } \\
\text { Steam supply was blanked at valve } \\
\text { MSS-V-17. Inside the fence. }\end{array}$ & No & No \\
\hline $200 \mathrm{~W}$ & b) & 552 & d & $\begin{array}{l}\text { 241-SX-401 Tank Farn Vapor Manifold } \\
\text { Condenser - Steam condensate and condenser } \\
\text { sampler line discharge to dry well (caisson). }\end{array}$ & 0.00 & Injection Well & $5 w 20$ & $\begin{array}{l}\text { E566,742.99 } \\
\text { N134,277.66 }\end{array}$ & $\begin{array}{l}\text { ELIMINATED 5/95. ADDED 2/95. } \\
\text { Steam supply eliminated in the late } \\
1970 \text { s. Inside the SX farm. }\end{array}$ & No & No \\
\hline $200 \mathrm{~W}$ & $\mathrm{D}$ & 553 & $d$ & $\begin{array}{l}\text { 241-SX-402 Tank Farn Vapor Manifold } \\
\text { Condenser - Condenser sanipler line } \\
\text { discharging to dry well (caisson). }\end{array}$ & 0.00 & Injection Well & $5 W 20$ & $\begin{array}{l}\text { ES66,742.77 } \\
\text { N134,383.74 }\end{array}$ & $\begin{array}{l}\text { ELIMINATED S/95. ADDED 2/95. } \\
\text { Steam supply was eliminated in the } \\
\text { late } 1970 \text { s. Inside the } S X \text { farm. }\end{array}$ & No & No \\
\hline $2001 \mathrm{~W}$ & $\mathrm{D}$ & 549 & d & $\begin{array}{l}\text { 241.SY Tank Fami - Slean pit discharges to } \\
\text { a caisson east of } 241-S Y-103 \text {. }\end{array}$ & 0.00 & Injection Well (nud leg) & $5 W 20$ & $\begin{array}{l}\text { Es66,871.58 } \\
\text { N134,532.66 }\end{array}$ & $\begin{array}{l}\text { ELIMINATED S/9S. ADDED 2/95. } \\
\text { Steam supply was blanked at valve } \\
\text { MSS-V-16. Northeast of the } \\
\text { exhauster (West of 103-SY). }\end{array}$ & No & No \\
\hline $200 \mathrm{~W}$ & D & 550 & d & $\begin{array}{l}\text { 241-SY Tank Farn - Stean pit. East of } 241 \text { - } \\
\text { SY-271. }\end{array}$ & 0.00 & Injection Well & $5 W 20$ & $\begin{array}{l}\text { E } 566,859.62 \\
\text { N134,577.79 }\end{array}$ & $\begin{array}{l}\text { ELIMINATED S/95. ADDED } 2 / 95 . \\
\text { Sicam supply was blanked at MSS. } \\
\text { V-16. }\end{array}$ & No & No \\
\hline $200 w^{\prime}$ & D & 234 & $d$ & $24 I Z$ Building - Main sleam line trap. & 0.05 & Injection Well & sw20 & $\begin{array}{l}\text { ES66,511.02 } \\
\text { N135,551.88 } \\
\end{array}$ & & No & Yes \\
\hline $200 \mathrm{~W}$ & D & 232 & cd & 24IZ Building - Eyewash/vafity shower. & 0.00 & Injection Well & $5 W 20$ & $\begin{array}{l}E 566,535.49 \\
N 135,535.14\end{array}$ & $\begin{array}{l}\text { East side of } 241 Z \text {. INACTIVE } \\
10 / 94 .\end{array}$ & Yes & Yes \\
\hline
\end{tabular}

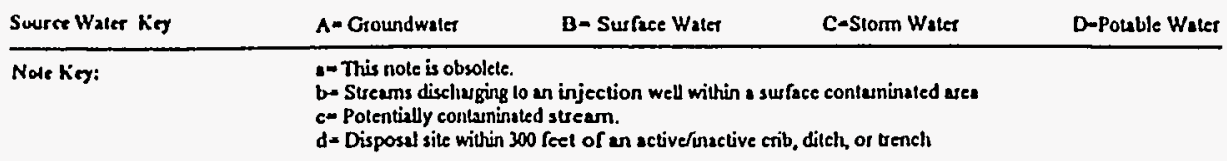


Table 3-1. Miscellaneous Streams Inventory

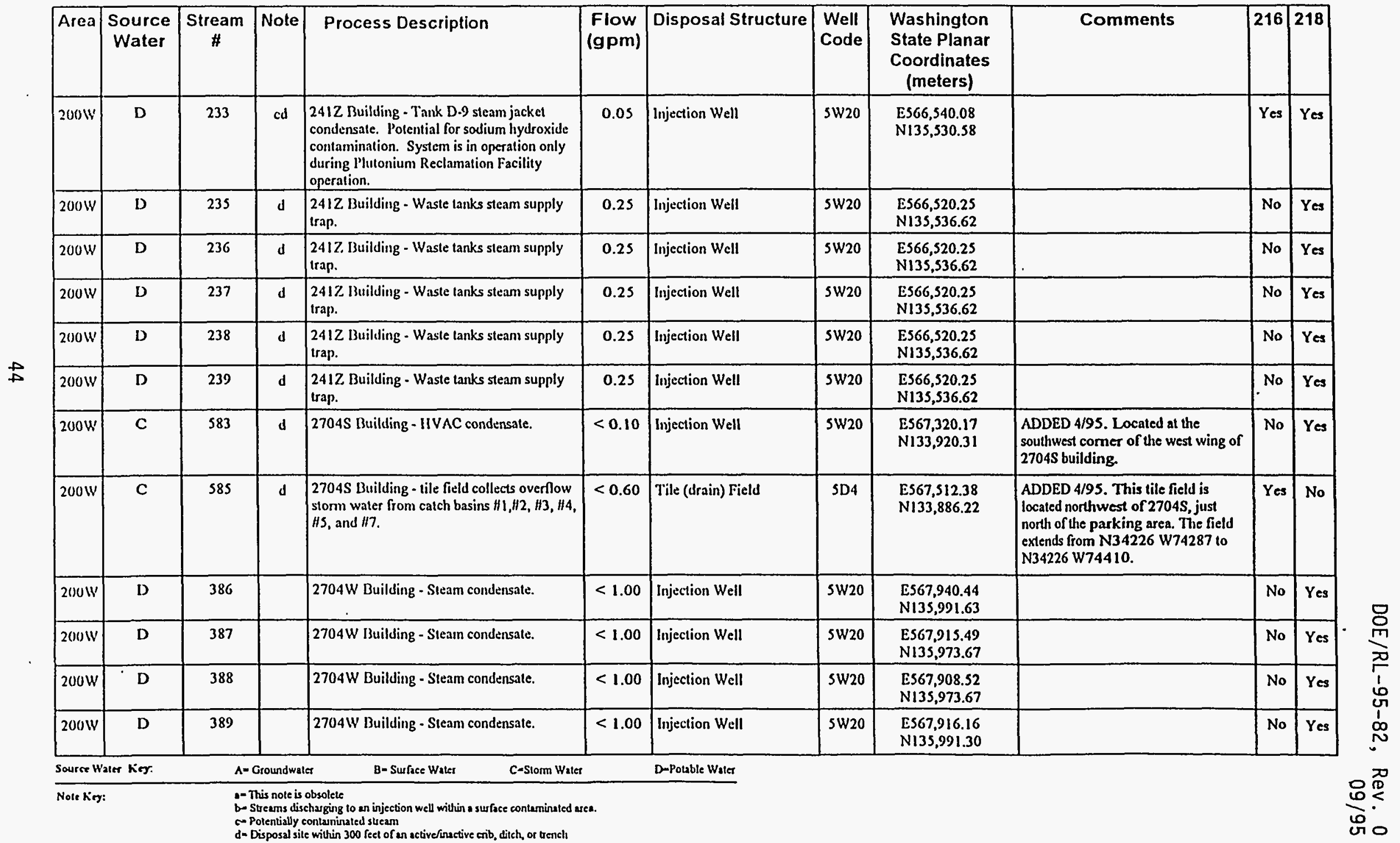


Table 3-1. Miscellaneous Streams Inventory

\begin{tabular}{|c|c|c|c|c|c|c|c|c|c|c|c|}
\hline Area & \begin{tabular}{|l|} 
Source \\
Water
\end{tabular} & $\begin{array}{c}\text { Stream } \\
\#\end{array}$ & Note & Process Description & $\begin{array}{c}\text { Flow } \\
\text { (gpm) }\end{array}$ & Disposal Structure & \begin{tabular}{|l|} 
Well \\
Code
\end{tabular} & $\begin{array}{c}\text { Washington } \\
\text { State Planar } \\
\text { Coordinates } \\
\text { (meters) }\end{array}$ & Comments & 216 & 218 \\
\hline $200 w$ & D & 390 & & 2704W Building - Steam condensate. & $<1.00$ & Injection Well & $5 W 20$ & $\begin{array}{l}\text { Es } 567,903.52 \\
N 136,003.94 \\
\end{array}$ & & No & Yes \\
\hline $200 \mathrm{w}$ & D & 391 & & 2704W Building - Steam condensate. & $<1.00$ & Injection Well & $5 W 20$ & $\begin{array}{l}\text { ES67,940.11 } \\
\mathrm{N} 136,003.94 \\
\end{array}$ & & No & Yes \\
\hline $200 \mathrm{~W}$ & $\mathrm{D}$ & 392 & & 2704W Building - Steam condensate. & $<1.00$ & Injection Well & $5 W 20$ & $\begin{array}{l}\text { ES67,918.82 } \\
\text { N136,003.94 } \\
\end{array}$ & & No & Yes \\
\hline $200 \mathrm{w}$ & D & 253 & d & $2704 \mathrm{Z}$ Building - Main steam line trap H02. & 0.05 & Injection Well & $5 W 20$ & $\begin{array}{l}\text { ES66,538.07 } \\
\text { N135,743.88 } \\
\end{array}$ & | ELIMINATED S/95. & No & No \\
\hline $200 \mathrm{~W}$ & D & 252 & d & $2704 Z$ Building - Main steam line trap HOI. & 0.05 & Injection Well & sw20 & $\begin{array}{l}\text { ES66,553.23 } \\
\text { N135,778.97 } \\
\end{array}$ & ELIMINATED 4/95. & No & No \\
\hline $200 \mathrm{w}$ & D & 27) & & 2707W Building - Steam condensute. & $<1.00$ & Injection Well & $5 W_{20}$ & $\begin{array}{l}\text { Es67,915.43 } \\
\text { N136,039.43 } \\
\end{array}$ & & No & Yes \\
\hline $200 \mathrm{~W}$ & D & 276 & & 2707W Building - Steam condensate. & $<1.00$ & Injection Well & $5 W 20$ & $\begin{array}{l}\text { ES67,940.31 } \\
\text { N136,039.43 } \\
\end{array}$ & & No & Yes \\
\hline $200 \mathrm{~W}$ & $\mathrm{D}$ & 277 & & 2707W Building - Steam condensate. & $<1.00$ & Injection Well & $5 \mathrm{~W} 20$ & $\begin{array}{l}\text { ES67,939.94 } \\
\text { N136,049.83 } \\
\end{array}$ & & No & Yes \\
\hline $200 \mathrm{w}$ & D & 278 & & 2707W Building - Steam condensate. & $<1.00$ & Injection Well & $5 W 20$ & $\begin{array}{l}\text { Es } 67,915.43 \\
\mathrm{~N} 136,049.08 \\
\end{array}$ & & No & Yes \\
\hline $200 \mathrm{w}$ & D & 280 & & 2707 W Building - Steam condensate. & $<1.00$ & Injection Well & $5 W 20$ & $\begin{array}{l}\text { Es67,922.49 } \\
\mathrm{N} 136,039.43 \\
\end{array}$ & & No & Yes \\
\hline $200 \mathrm{~W}$ & D & 536 & & 2707W Building - Steam condensatc. & 1.00 & Injection Well & $5 W_{20}$ & $\begin{array}{l}\text { Es67,908.38 } \\
\mathrm{N} 136,046.86 \\
\end{array}$ & ADDED 2/95. West of $2707 \mathrm{~W}$ & No & Yes \\
\hline $200 \mathrm{~W}$ & c & 537 & & 2707W Building - Stonn water run-olf. & 1.00 & Injection Well & SD2 & $\begin{array}{l}\text { E } 367,900.58 \\
N 136,040.54\end{array}$ & $\begin{array}{l}\text { ADDED 2/95. East of southwest } \\
\text { comer of 2707W }\end{array}$ & Yes & Yes \\
\hline $200 \mathrm{w}$ & D & 281 & & 2713 W Building - Steam condensate. & $<1.00$ & Injection Well & $5 W 20$ & $\begin{array}{l}\text { Es67,853.69 } \\
\text { N136,192.19 }\end{array}$ & $\begin{array}{l}\text { CORRECTED 2/95. Coordinates. } \\
\text { Soulliwest comer of } 2713 \text { w. }\end{array}$ & No & Yes \\
\hline $2001 \mathrm{~W}$ & $\mathrm{D}$ & 282 & & 27I3W IBuilding - Stean condensale. & $<1.00$ & Injection Well & $5 W 20$ & $\begin{array}{l}\text { E567,853.59 } \\
\text { N136,208.94 }\end{array}$ & $\begin{array}{l}\text { CORRECTED 2/95. Coordinates. } \\
\text { Northwest comer } 2713 \mathrm{~W} \text {. }\end{array}$ & No & $Y_{e s}$ \\
\hline
\end{tabular}


Table 3-1. Miscellaneous Streams Inventory

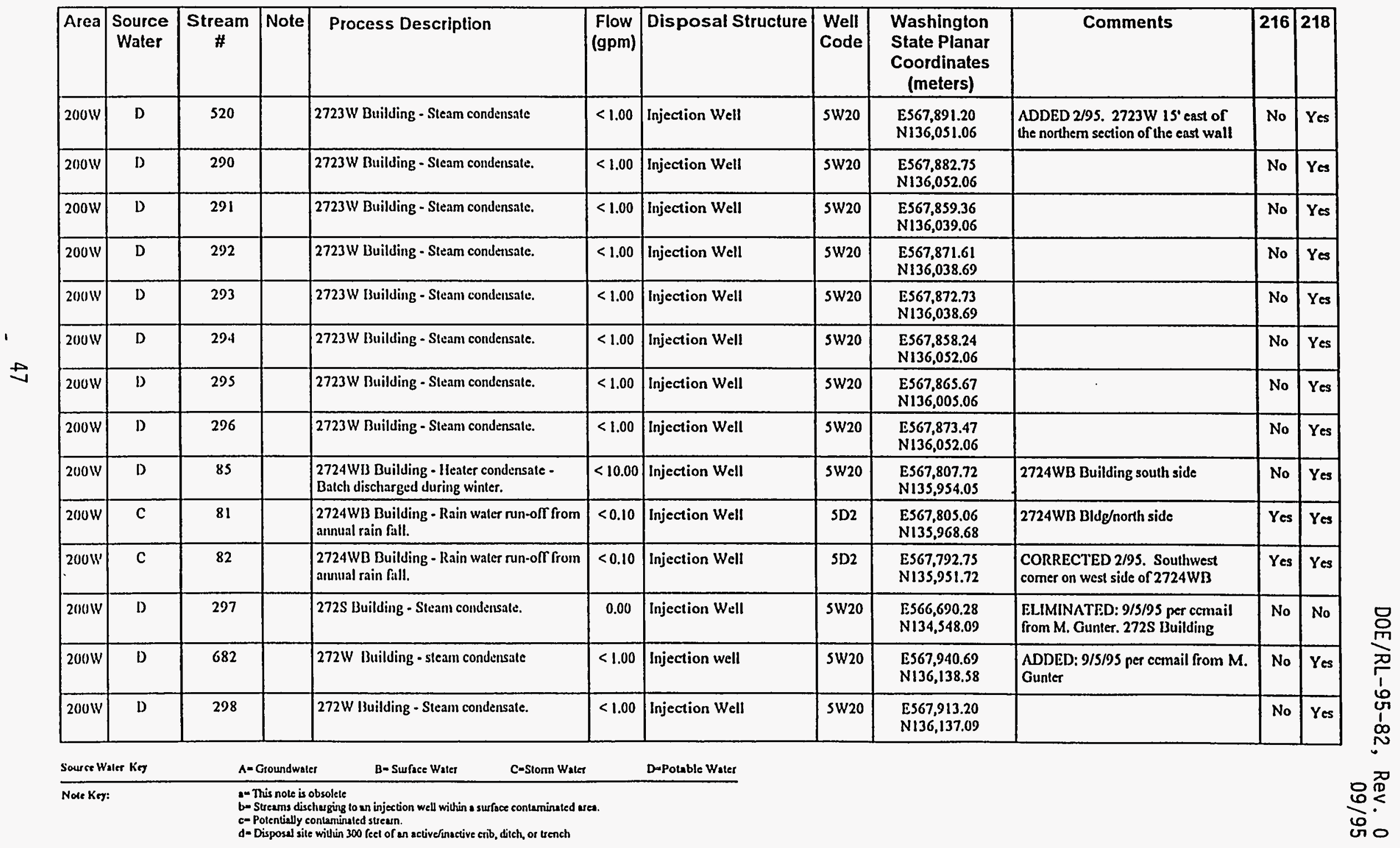


Table 3-1. Miscellaneous Streams Inventory

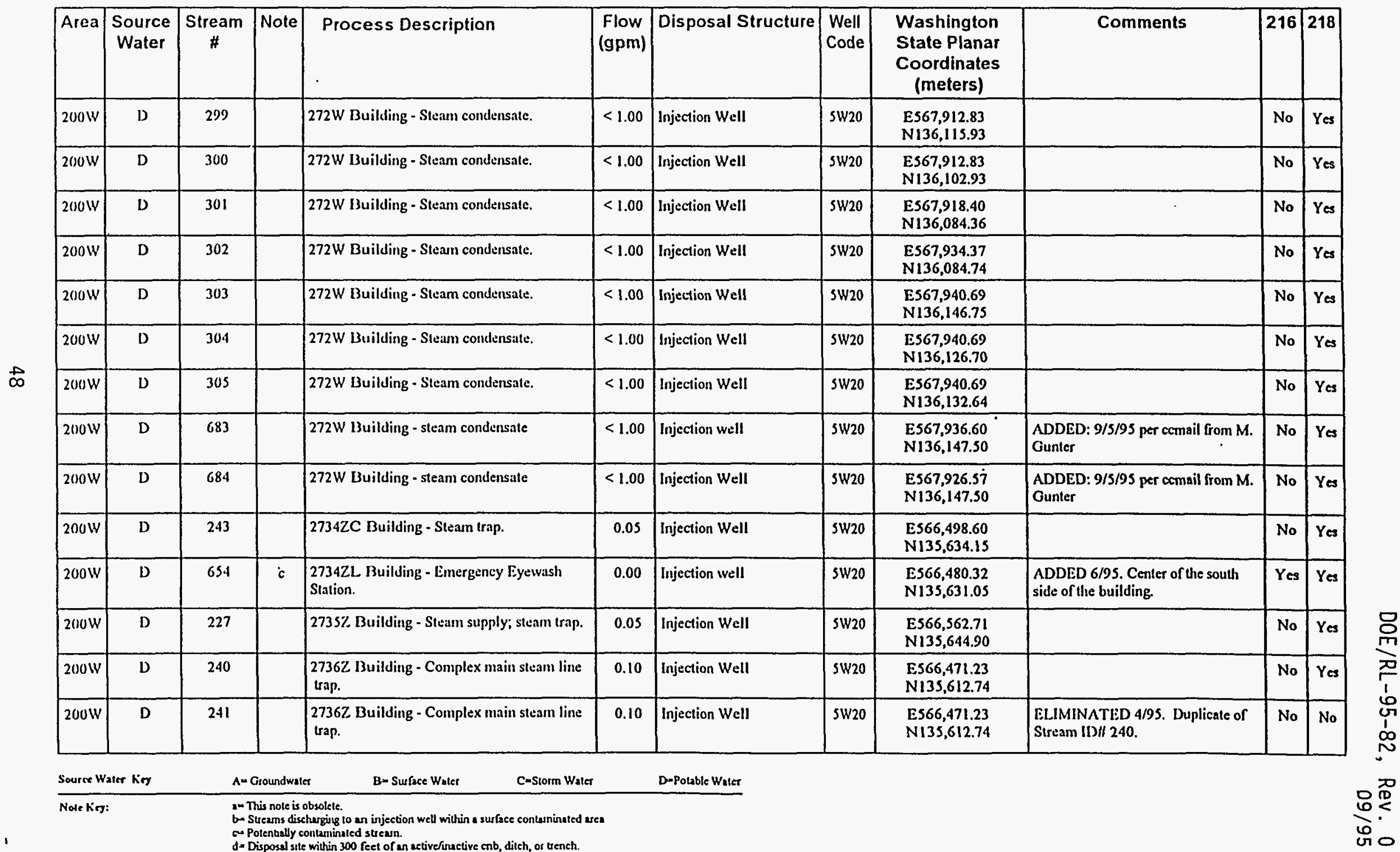


Table 3-1. Miscellaneous Streams Inventory

\begin{tabular}{|c|c|c|c|c|c|c|c|c|c|c|c|}
\hline Area & $\begin{array}{l}\text { Source } \\
\text { Water }\end{array}$ & $\begin{array}{l}\text { Stream } \\
\#\end{array}$ & Note & Process Description & $\begin{array}{c}\text { Flow } \\
\text { (gpm) }\end{array}$ & Disposal Structure & \begin{tabular}{l|} 
Well \\
Code
\end{tabular} & $\begin{array}{c}\text { Washington } \\
\text { State Planar } \\
\text { Coordinates } \\
\text { (meters) } \\
\end{array}$ & Comments & 216 & 218 \\
\hline $200 \mathrm{w}$ & c & 244 & & $27362 C$ Building - Storn drain. & $<0.01$ & Injection Well & SD2 & $\begin{array}{l}\text { ES66,465.27 } \\
\text { N135,560.90 }\end{array}$ & & Yes & Yes \\
\hline $200 \mathrm{w}$ & D & 534 & & $\begin{array}{l}273 \text { W Building - Sanitary water reccived } \\
\text { from } 277 \text { W Building. }\end{array}$ & $<1.00$ & Injection Well & $5 \mathrm{~W} 20$ & $\begin{array}{l}\text { ES67,831.51 } \\
\text { N136,119.64 }\end{array}$ & $\begin{array}{l}\text { ADDED 2/95. } 10 \text { fect east of } 273 \mathrm{~W} \\
\text { towards north end. }\end{array}$ & Yes & Yes \\
\hline $200 \mathrm{~W}$ & $\overline{C D}$ & 506 & & $\begin{array}{l}\text { 274W Building - Steam condensate and } \\
\text { stom water. }\end{array}$ & $<5.00$ & Injection Well & SD2, & $\begin{array}{l}\text { ES67,827.79 } \\
\text { N136,146.75 }\end{array}$ & $\begin{array}{l}\text { ADDED 12/94. Adjacent to south } \\
\text { wall, } \sim 50^{\prime} \text { west of southeast comer of } \\
\text { building } 274 \mathrm{~W}\end{array}$ & Yes & Yes \\
\hline $2000 \mathrm{~W}$ & D & 306 & & 274W Btuilding - Steam condensale. & $<5.00$ & Injection Well & $5 W_{20}$ & $\begin{array}{l}E S 67.873 .84 \\
N 136,151.95\end{array}$ & $\begin{array}{l}\text { Adjacent to building, } \sim 10 \text { north of } \\
\text { southwest cormer }\end{array}$ & No & Yes \\
\hline $200 \mathrm{~W}$ & D & sos & & 274 W Building - Stean condensale. & $<5.00$ & Injection Well & $5 w_{20}$ & $\begin{array}{l}\text { ES67,843.39 } \\
\text { N136,148.98 }\end{array}$ & $\begin{array}{l}\text { ADDED 12/94. Adjacent to the } \\
\text { 274W building at the southwest } \\
\text { comer, on the south wall }\end{array}$ & No & $\overline{\text { Yes }}$ \\
\hline $200 \mathrm{w}$ & $\bar{D}$ & 507 & & 274W Building - Steam condesate. & $<5.00$ & Injection Well & $5 w 20$ & $\begin{array}{l}\text { ES67,867.53 } \\
\text { N136,148.98 }\end{array}$ & $\begin{array}{l}\text { ADDED 12/94. Approximately } 10 \\
\text { west of the southeast comer of } \\
\text { building } 274 W, \text { adjacent to south } \\
\text { wall. }\end{array}$ & No & Yes \\
\hline $2001 \mathrm{~W}$ & D & 543 & & $\begin{array}{l}\text { 275W Building - Steam condensale from } \\
\text { three traps of of the overhead steann line. }\end{array}$ & $<5.00$ & Injection Well & $5 w_{20}$ & $\begin{array}{l}\text { ES67,814.76 } \\
\text { Ni36,150.82 }\end{array}$ & $\begin{array}{l}\text { ELIMINATED 4/95. No steam lines } \\
\text { currently run to the building. } \\
\text { ADDED 2/95. Approximately 60n } \\
\text { off the soutlieast comer of } 275 \mathrm{~W} \text {. }\end{array}$ & No & No \\
\hline $2010 \mathrm{WV}$ & $\bar{D}$ & 307 & & 275W Bsuilding - Steam condensalc. & $<5.00$ & Injection Well & $\overline{5 W 20}$ & $\begin{array}{l}\text { ES67,771.86 } \\
\text { N136,122.07 }\end{array}$ & $\begin{array}{l}\text { ELIMINATED 4/95. No steam lines } \\
\text { currenlly run to the building. 275W } \\
\text { Building }\end{array}$ & No & No \\
\hline $200 w$ & D & 6.31 & & $\begin{array}{l}277 \text { W Building - Steam condensate from } \\
\text { building lieat is discharged to this disposal } \\
\text { Irencl. }\end{array}$ & $<0.10$ & Trench & $\bar{N} / \mathrm{A}$ & $\begin{array}{l}\text { E567,779.51 } \\
\text { N136,095.13 }\end{array}$ & $\begin{array}{l}\text { ADDED 4/9S. This trench will be } \\
\text { west of } 277 W \text {, located in the } \\
\text { equipment laydown area. The } \\
\text { structure will be completed 10/95. }\end{array}$ & Yes & No \\
\hline $200 \mathrm{w}$ & $\bar{D}$ & 80 & c & $\begin{array}{l}\text { 277W Fabrication Shop - Condensale from } \\
\text { conpressor. }\end{array}$ & $<5.00$ & Injection Well & $5 W 20$ & $\begin{array}{l}\text { ES } 567,842.65 \\
N 136,097.73\end{array}$ & & Yes & Yes \\
\hline
\end{tabular}

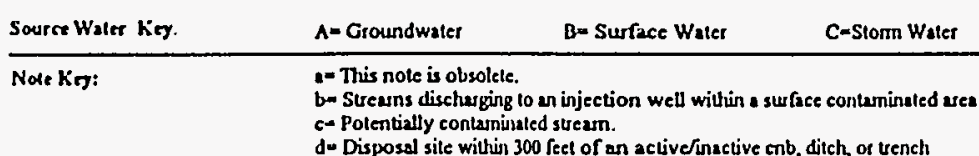


Table 3-1. Miscellaneous Streams Inventory

\begin{tabular}{|c|c|c|c|c|c|c|c|c|c|c|c|}
\hline Area & $\begin{array}{l}\text { Source } \\
\text { Water }\end{array}$ & $\begin{array}{c}\text { Stream } \\
\#\end{array}$ & Note & Process Description & $\begin{array}{l}\text { Flow } \\
\text { (gpm) }\end{array}$ & Disposal Structure & $\begin{array}{l}\text { Well } \\
\text { Code }\end{array}$ & $\begin{array}{c}\text { Washington } \\
\text { State Planar } \\
\text { Coordinates } \\
\text { (meters) } \\
\end{array}$ & Comments & 216 & 218 \\
\hline $2001 \mathrm{~W}$ & $\mathrm{D}$ & 77 & & $\begin{array}{l}\text { 277W Fabrication Shop - Conderssate from } \\
\text { two IIVAC units. }\end{array}$ & 0.00 & Injection Well & sw20 & $\begin{array}{l}\text { ES67,859.67 } \\
\text { Ni36,105.22 }\end{array}$ & $\begin{array}{l}\text { ELIMINATED: } 9 / 5 / 95 \text {, injection } \\
\text { well has been paved over per ccmail } \\
\text { from M. Gunler }\end{array}$ & No & No \\
\hline $200 \mathrm{~W}$ & $\bar{D}$ & 78 & & $\begin{array}{l}\text { 277W Fabrication Shop - Condensilte from } \\
\text { two IIVAC units. }\end{array}$ & 0.00 & Injection Well & sw20 & $\begin{array}{l}\text { ES67,917.57 } \\
\text { N136,105.37 }\end{array}$ & $\begin{array}{l}\text { ELIMINATED: } 9 / 5 / 95 \text { injection } \\
\text { well has been paved over per ccmail } \\
\text { from M. Gunler }\end{array}$ & No & No \\
\hline $2000 \mathrm{~W}$ & D) & 76 & & $\begin{array}{l}277 \text { Fabrication Shop - Condensiate from } \\
\text { thuilding heater and spray pan water. }\end{array}$ & $<1.00$ & Injection Well & sw20 & $\begin{array}{l}\text { E } 567,815.51 \\
\text { N136,092.92 }\end{array}$ & $\begin{array}{l}\text { CORRECTED 2/95. Located steam } \\
\text { line on right side of door } \$ 06 \text { that } \\
\text { runs over to the southside of } 273 \mathrm{~W} \text {. }\end{array}$ & No & Yes \\
\hline $200 \mathrm{w}$ & 1) & 75 & & $\begin{array}{l}277 \text { W Falurication Shop - Condensale from } \\
\text { building heater. }\end{array}$ & $<1.00$ & Injection Well & $5 w 20$ & $\begin{array}{l}\text { Es67,887.95 } \\
\text { N136,079.54 }\end{array}$ & Soulleast side of building 277 & No & Yes \\
\hline $200 \mathrm{w}$ & D & 79 & & $\begin{array}{l}\text { 277W Fabrication Shop - Sanitary water } \\
\text { (pressure regulating valve relief). }\end{array}$ & $<0.01$ & Injection Well & $5 w_{20}$ & $\begin{array}{l}\text { ES68,176.68 } \\
\text { N136,077.06 }\end{array}$ & $\begin{array}{l}\text { ELIMINATED 2/95. Stream could } \\
\text { nol be verified. }\end{array}$ & No & No \\
\hline $200 \mathrm{~W}$ & $D$ & 637 & & $\begin{array}{l}\text { 283W Building - Building heater steam traps } \\
\text { located inside of the building discharge to an } \\
\text { injection well on the east side exterior of the } \\
\text { building. }\end{array}$ & $<0.01$ & Injection well & $5 w_{20}$ & $\begin{array}{l}\text { E567,689.07 } \\
\text { N136,081.94 }\end{array}$ & $\begin{array}{l}\text { ADDED 6/95. Located on the east } \\
\text { side of } 283 \mathrm{~W} \text {. }\end{array}$ & No & Yes \\
\hline $200 \mathrm{~W}$ & D & 636 & & $\begin{array}{l}\text { 284W- Steam Iraps } 2 Q-Y \text { ard-MSS-TRP- } \\
001,010,127,128,065 \text { discharge to one } \\
\text { injection well located belind the new } 200 \mathrm{~W} \\
\text { package boilers. }\end{array}$ & $<0.01$ & Injection well & $5 w_{20}$ & $\begin{array}{l}\text { E567,646.71 } \\
\text { N135,959.94 }\end{array}$ & $\begin{array}{l}\text { CORRECTED: } 7 / 6 / 95 \text { PER ce:mail } \\
\text { from M. Gunter dated 6/29/95. } \\
\text { ADDED 6/95. These traps discharge } \\
\text { to the same injection well behind the } \\
200 \text { W package boiler. }\end{array}$ & No & Yes \\
\hline $200 \mathrm{w}$ & 13 & 471 & & 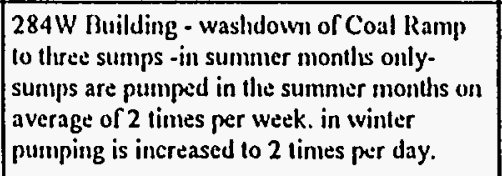 & $<0.50$ & $\begin{array}{l}\text { Manmade depression } \\
\text { (Pond is dry nost times) }\end{array}$ & $N / \Lambda$ & $\begin{array}{l}\text { E567,458.01 } \\
N 135,983.86\end{array}$ & \begin{tabular}{|l|} 
ELIMINATED 4/95. Coal ramp \\
washdown is no longer used. \\
ADDIDD lo/94. End of Coal Ramp \\
at Coal Loading Station across \\
Railroad tracks
\end{tabular} & $\overline{\text { No }}$ & No \\
\hline $200 w$ & D & 385 & & 284W Hligh Water Tank overllow. & 5.00 & Open Trench & $N / A$ & $\begin{array}{l}\text { E567,976.43 } \\
\text { N136,213.09 }\end{array}$ & $\begin{array}{l}\text { Water is potable and for general use } \\
\text { in the } 200 \mathrm{~W} \text { area. }\end{array}$ & Yes & No \\
\hline $200 \mathrm{~W}$ & $\bar{D}$ & 251 & & $\begin{array}{l}\text { 29022. Building - Iligh water tower steam } \\
\text { trap. }\end{array}$ & 0.02 & Injection Well & $s w 20$ & $\begin{array}{l}\text { ES } 566,440.52 \\
\text { N135,737.54 } \\
\end{array}$ & & No & Yes \\
\hline
\end{tabular}


Table 3-1. Miscellaneous Streams Inventory

\begin{tabular}{|c|c|c|c|c|c|c|c|c|c|c|c|}
\hline Area & $\begin{array}{c}\text { Source } \\
\text { Water }\end{array}$ & $\begin{array}{c}\text { Stream } \\
\#\end{array}$ & Note & Process Description & $\begin{array}{c}\text { Flow } \\
\text { (gpm) }\end{array}$ & Disposal Structure & $\begin{array}{l}\text { Well } \\
\text { Code }\end{array}$ & $\begin{array}{l}\text { Washington } \\
\text { State Planar } \\
\text { Coordinates } \\
\text { (meters) }\end{array}$ & Comments & 216 & 218 \\
\hline $200 \mathrm{~W}$ & D & 551 & d & $\begin{array}{l}\text { 296.S-15 (Sludge Cooler) - Steam heater } \\
\text { discharging to a dry well (caisson). }\end{array}$ & 0.00 & Injection Well & $5 W 20$ & $\begin{array}{l}\text { Es66,764.50 } \\
\text { N134,190.01 }\end{array}$ & $\begin{array}{l}\text { ELIMINATED S/95. ADDED 2/95. } \\
\text { Steam supply was blanked at valve } \\
\text { MSS-V-17. Inside the SX farm. }\end{array}$ & No & No \\
\hline $200 \mathrm{~W}$ & D & 263 & b & $\begin{array}{l}\text { Condensate discharged to french drain } 216- \\
Z-15 \text {. }\end{array}$ & 1.00 & Injection Well & $5 W 20$ & $\begin{array}{l}\text { E566,465.27 } \\
\text { N135, } 560.90\end{array}$ & $\begin{array}{l}\text { Potential historical contamination } \\
\text { underground at discharge location. }\end{array}$ & No & Yes \\
\hline $200 \mathrm{~W}$ & D & 468 & & $\begin{array}{l}\text { DACS Trailer - IIVAC - Condensule from } \\
10 \text { ton IIVAC condenser. }\end{array}$ & $<0.01$ & Injection Well & $5 W 20$ & $\begin{array}{l}E 566,821.09 \\
N 134,638.33\end{array}$ & $\begin{array}{l}\text { ADDED 11/94. Approximately } 10 \\
\text { feet north of the DACS trailer, } 12 \\
\text { inches below grade. }\end{array}$ & No & Yes \\
\hline $200 w$ & $\mathrm{D}$ & 51 & $d$ & $\begin{array}{l}\text { Ice llouse adjacent to W-IS Shect Metal } \\
\text { Sliop - Water jug rinsate batch discharged } \\
\text { during cleaning activities. A mixture of I } \\
\text { cup bleach and } 1 / 4 \text { cup NallcO3 (sodium } \\
\text { bicarbonate) to } 20 \text { gallons of water is used } 5 \\
\text { days a week to clean/sterilize the jug. } \\
\end{array}$ & 0.01 & Injection Well & $5 W 20$ & $\begin{array}{l}\text { ES } 67,302.95 \\
\text { N135,894.20 }\end{array}$ & $\begin{array}{l}\text { ELIMINATED 4/95. Discharge was } \\
\text { rerouted to the sanitary sewer. } \\
\text { CORRECTED 2/95. Comment and } \\
\text { contact added. }\end{array}$ & No & No \\
\hline $200 \mathrm{~W}$ & D & 39 & & Laborer's Storage - Steam condensate. & 0.05 & lnjection Well & $5 W 20$ & $\begin{array}{l}\text { E567,258.21 } \\
\text { N135,833.50 } \\
\end{array}$ & CORRECTED 2/95. Contact added. & No & Yes \\
\hline $200 \mathrm{~W}$ & $\mathrm{c}$ & 523 & & MO028 Building - Stonm water run-olf. & 1.00 & Injection Well & SD2 & $\begin{array}{l}\text { E567,258.76 } \\
\text { N133,854.37 }\end{array}$ & $\begin{array}{l}\text { ADDED 2/95. } 251 \text { north of the } \\
\text { northeast comer of MO028 }\end{array}$ & Yes & Yes \\
\hline $200 \mathrm{~W}$ & D & 45 & & M0716 Building - Stean condensate. & 0.05 & Injection Well & $5 W 20$ & $\begin{array}{l}\text { E567,270.25 } \\
\text { N135,894.79 }\end{array}$ & CORRECTED 2/95. Contact added. & No & Yes \\
\hline $200 W$ & $\mathrm{CD}$ & 44 & & $\begin{array}{l}\text { MO716 Paint Shop - Steam condensate; also } \\
\text { receives stom water null-oll. }\end{array}$ & 0.05 & Injection Well & SD2, & $\begin{array}{l}\text { E567,250.58 } \\
\text { N135,889.89 }\end{array}$ & $\begin{array}{l}\text { CORRECTED 2/95. Comment and } \\
\text { contact added. This trap is in a fenced } \\
\text { area west of the painting booth. }\end{array}$ & Yes & Yes \\
\hline $200 w$ & D & 222 & b & $\begin{array}{l}\text { S/S.VSY/242S Complex - Steain } \\
\text { condensate; steam condensate discharged } \\
\text { year-round to caisson localed within a } \\
\text { surface contaminated area. }\end{array}$ & $<0.00$ & Injection Well & sw20 & $\begin{array}{l}\text { Es66,902.16 } \\
\text { N134,358.78 }\end{array}$ & $\begin{array}{l}\text { ELIMIN TTED S/9S. No longer } \\
\text { receives waste per Bowman } 8 / 95 \text {. } \\
\text { CORRECTED 2/25. Comments and } \\
\text { contacts. Disposal area will be } \\
\text { relocated to an uncontaminated area. } \\
\text { Outside perimeter fence, on east side } \\
\text { of } 242-S \text {. Not active. }\end{array}$ & No & No \\
\hline Source W & Mer Key. & $A-$ & oundwate & B-Surface Water & & D-Poluble Water & & & & & \\
\hline
\end{tabular}


Table 3-1. Miscellaneous Streams Inventory

\begin{tabular}{|c|c|c|c|c|c|c|c|c|c|c|c|}
\hline Area & $\begin{array}{l}\text { Source } \\
\text { Water }\end{array}$ & Stream & Note & Process Description & $\begin{array}{c}\text { Flow } \\
\text { (gpm) }\end{array}$ & Disposal Structure & $\begin{array}{l}\text { Well } \\
\text { Code }\end{array}$ & $\begin{array}{l}\text { Washington } \\
\text { State Planar } \\
\text { Coordinates } \\
\text { (meters) }\end{array}$ & Comments & 216 & 218 \\
\hline $200 w$ & $\mathrm{D}$ & 221 & & $\begin{array}{l}\text { S/SX/SY/242S CompleX - Stenm } \\
\text { condensate; steam condensate discharges } \\
\text { year-round from steam trap on live steam } \\
\text { line. }\end{array}$ & $<0.00$ & Injection Well & sw20 & $\begin{array}{l}\text { ES66,857.99 } \\
\text { Ni34,619.23 }\end{array}$ & $\begin{array}{l}\text { ELIMINATED S/95. No longer } \\
\text { receives waste per Mark Bowman } \\
\text { 8/95. CORRECTED 2/95. } \\
\text { Comments and contacts. } \\
\text { CORRECTED 12/94. Area was } \\
\text { corrected. Oulside perimeter fence, } \\
\text { north of SY Farm. Not active.. }\end{array}$ & No & No \\
\hline $2001 \mathrm{~V}$ & D) & 42 & & Skid Shack - Steam condensate. & 0.05 & Injection Well & $5 w 20$ & $\begin{array}{l}E 567,234.56 \\
\text { N135,858.34 }\end{array}$ & $\begin{array}{l}\text { CORRECTED } 2 / 95 \text {. Comment and } \\
\text { contact added. West of W-20 } \\
\text { Pipefitter's shop. }\end{array}$ & No & Yes \\
\hline $200 \mathrm{~W}$ & 1) & 137 & & $\begin{array}{l}\text { Steam Trap - 2Q-Yard-MSS-TRRP-002 } \\
\text { (formerly Steam trap /102)-Steam } \\
\text { condensate. }\end{array}$ & $<1.00$ & Injection Well & $5 W 20$ & $\begin{array}{l}\text { ES67,705.01 } \\
\text { N135,798.57 }\end{array}$ & $\begin{array}{l}\text { Steam line on Beloit street from } \\
\text { Powerhouse across } 19 \text { th to REDOX. } \\
\text { 19th and Beloit }\end{array}$ & No & Yes \\
\hline $200 \mathrm{~W}$ & 1) & 138 & b & $\begin{array}{l}\text { Steam Trap 2Q - Yard-MSS-TRP- } 003,063 \\
\text { - Steam condensate disclarged to french } \\
\text { drain located on the perimeter of a surface } \\
\text { contaminated area. }\end{array}$ & $<1.00$ & Injection Well & $5 w 20$ & $\begin{array}{l}\text { ES67,751.60 } \\
\text { N135,448.22 }\end{array}$ & & No & Yes \\
\hline $200 \mathrm{~W}$ & $\mathrm{D}$ & 139 & & Steam Trap 2Q - Yard-MSS-TRP-004. & $<1.00$ & Injection Well & $5 W 20$ & $\begin{array}{l}\text { Es67,752.19 } \\
\text { N135,213.56 }\end{array}$ & $\begin{array}{l}\text { Steam line on Beloit street from } \\
\text { Powerhouse across 19th to REDOX. }\end{array}$ & No & Yes \\
\hline $200 \mathrm{w}$ & $\mathrm{D}$ & 140 & & Steam Trap 2Q - Yard-MSS-TRR'-00S. & $<1.00$ & Injection Well & sw20 & $\begin{array}{l}\text { ES67,677.37 } \\
\text { N134,658.73 }\end{array}$ & $\begin{array}{l}\text { Steam line on Beloit street from } \\
\text { Powethouse across } 19 \text { th to REDOX. }\end{array}$ & $\overline{\text { No }}$ & Yes \\
\hline $2000 \mathrm{~W}$ & $\mathrm{D}$ & 141 & b & $\begin{array}{l}\text { Steam Trap 2Q - Yard-MSS-TRP.006- } \\
\text { Steam condensale discharged to a french } \\
\text { drain located on the perimeter of a surface } \\
\text { contaminated area. }\end{array}$ & $<1.00$ & Injection Well & $5 W 20$ & $\begin{array}{l}\text { ES67,645.86 } \\
\text { NI35,079.21 }\end{array}$ & $\begin{array}{l}\text { Trap is under Steam Utilities } \\
\text { Organization. Beluind UO3 }\end{array}$ & No & Yes \\
\hline $200 \mathrm{~W}$ & D) & 142 & b & $\begin{array}{l}\text { Steam Trap } 2 Q \text { - Yard-MSS-TRP-007- } \\
\text { Steam condensate discharged to a french } \\
\text { drain localed on the perimeler of a surface } \\
\text { contaminated area. }\end{array}$ & $<1.00$ & Injection Well & $5 W 20$ & $\begin{array}{l}\text { ES67,569.94 } \\
\text { N134,969.31 }\end{array}$ & $\begin{array}{l}\text { Trap is under Steam Utilities } \\
\text { Organization. Comer of UO3 off } \\
\text { I Gth street }\end{array}$ & No & Yes \\
\hline $200 \mathrm{~W}$ & $\mathrm{D}$ & 143 & & Steam Trap 2Q - Yard-MSS-TRP-008. & $<1.00$ & Injection Well & $5 W 20$ & $\begin{array}{l}\text { ES } 57,570.00 \\
\text { N134,944.93 }\end{array}$ & $\begin{array}{l}\text { Steam line on Beloit street from } \\
\text { Powerliouse across } 19 \text { th to REDOX. } \\
\text { Opposite comer of } H 07 \text { trap }\end{array}$ & No & Yes \\
\hline \multicolumn{12}{|c|}{ Source Waler Key } \\
\hline \multicolumn{12}{|c|}{ 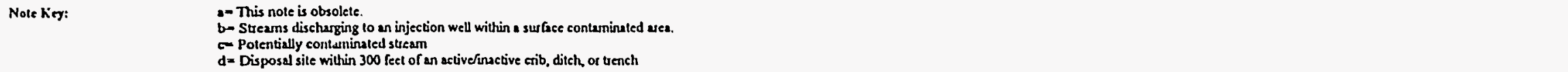 } \\
\hline
\end{tabular}


Table 3-1. Miscellaneous Streams Inventory

\begin{tabular}{|c|c|c|c|c|c|c|c|c|c|c|c|}
\hline Area & $\begin{array}{l}\text { Source } \\
\text { Water }\end{array}$ & $\begin{array}{l}\text { Stream } \\
\#\end{array}$ & Note & Process Description & $\begin{array}{l}\text { Flow } \\
\text { (gpm) }\end{array}$ & Disposal Structure & $\begin{array}{l}\text { Well } \\
\text { Code }\end{array}$ & $\begin{array}{l}\text { Washington } \\
\text { State Planar } \\
\text { Coordinates } \\
\text { (meters) }\end{array}$ & Comments & 216 & 218 \\
\hline $200 w$ & D & 144 & & Steam Trap 2Q - Yard-MSS-TRP-009. & $<1.00$ & Injection Well & $5 w 20$ & $\begin{array}{l}\text { E567,432.79 } \\
\text { N134,975.07 }\end{array}$ & $\begin{array}{l}\text { Steam line on Beloit street from } \\
\text { Powerhouse across } 19 \text { th to REDOX. } \\
\text { Of } 16 \text { th junction to } 241 U \text { and } \\
\text { Redox line. }\end{array}$ & No & Yes \\
\hline $200 \mathrm{~W}$ & $\mathbf{D}$ & 145 & b & $\begin{array}{l}\text { Steam Trap } 2 Q \text { - Yard-MSS-TRP.-014- } \\
\text { Steam condensate discharged to a french } \\
\text { drain that is located in a surface } \\
\text { contaminated area. }\end{array}$ & $<1.00$ & Injection Well & sw20 & $\begin{array}{l}\text { ES67,265.88 } \\
\text { N134,688.19 }\end{array}$ & $\begin{array}{l}\text { Trap is under Steam Utilities } \\
\text { Organization. Ofr road next to RR } \\
\text { track to REDOX }\end{array}$ & No & Yes \\
\hline $200 w$ & D & 146 & b & $\begin{array}{l}\text { Sleam Trap 2Q - Yard-MSS-TRP-015, } 064 \text { - } \\
\text { Sleam condensate. }\end{array}$ & $<1.00$ & Injection Well & $5 W 20$ & $\begin{array}{l}\text { Es67,266.58 } \\
\text { N134,404.78 }\end{array}$ & $\begin{array}{l}\text { CORRECTED: 7/6/95 PER } \\
\text { CC:MAIL from M. Gunter. Trap is } \\
\text { under Steam Utilities Organization. } \\
\text { On redox line near trap } \$ 14\end{array}$ & No & Yes \\
\hline $200 \mathrm{w}$ & D & 147 & & Steam Irap 2Q - Yard-MSS-TRP-016. & $<1.00$ & Injection Well & $5 W 20$ & $\begin{array}{l}\text { ES67,267.39 } \\
\text { N134,102.70 }\end{array}$ & $\begin{array}{l}\text { Steam line on Beloit street from } \\
\text { Powerhouse across } 19 \text { th to REDOX. } \\
\text { Comer of fence outside REDOX }\end{array}$ & No & Yes \\
\hline $2001 \mathrm{~W}$ & $\mathbf{D}$ & 148 & & Steam 'Trap 2Q - Yard-MSS-TRP-017. & $<1.00$ & Injection Well & $5 W 20$ & $\begin{array}{l}\text { Es67,374.48 } \\
\text { N } 133,880.51\end{array}$ & $\begin{array}{l}\text { Steam line on Beloit street from } \\
\text { Powerhouse across } 19 t h \text { to REDOX. } \\
\text { In front of 222-S Lab }\end{array}$ & No & Yes \\
\hline $2001 \mathrm{~W}$ & D & 136 & & Steam Trap 2Q - Yard-MSS-TRP-023. & $<1.00$ & Injection Well & sw20 & $\begin{array}{l}\text { ES } 67,628.75 \\
\text { N135,828.85 }\end{array}$ & $\begin{array}{l}\text { Steam line from Powerhouse off of } \\
\text { 19th street. }\end{array}$ & No & Yes \\
\hline $200 \mathrm{~W}$ & $\mathbf{D}$ & 134 & & Steam Trap 2Q - Yard-MSSS-TRL-024. & $<1.00$ & Injection Well & $5 W 20$ & $\begin{array}{l}\text { E567,522.16 } \\
\text { N135,798.11 }\end{array}$ & $\begin{array}{l}\text { Steam line from Powerhouse off of } \\
19 \text { th street. } 19 \text { th street and the end of } \\
\text { RR track }\end{array}$ & No & Yes \\
\hline $200 \mathrm{~W}$ & D & 135 & & Steam Trap 2Q - Yard-MSS-TRP-025. & $<1.00$ & Injection Well & $5 W 20$ & $\begin{array}{l}\text { E567,461.22 } \\
\text { N135,797.96 }\end{array}$ & $\begin{array}{l}\text { Steam line from Powerhouse off of } \\
19 \text { th street. Next to ash disposal on } \\
19 \text { th }\end{array}$ & No & Yes \\
\hline $2000 \mathrm{~W}$ & D & 132 & & Stean Trap 2Q - Yard-MSS-T'RP'-026,027. & $<1.00$ & Injection Well & $5 W 20$ & $\begin{array}{l}\text { ES67,445,98 } \\
\text { N } 135,797.92\end{array}$ & $\begin{array}{l}\text { Steam line from Powerhouse off of } \\
19 \|_{1} \text { street. Front of trailer between } \\
\text { powerhouse and PFP }\end{array}$ & No & Yes \\
\hline
\end{tabular}

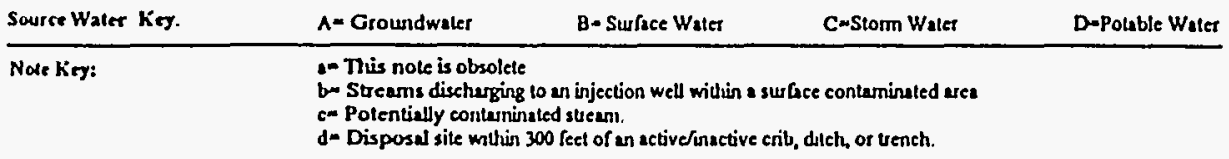


Table 3-1. Miscellaneous Streams Inventory

\begin{tabular}{|c|c|c|c|c|c|c|c|c|c|c|c|}
\hline Area & $\begin{array}{c}\text { Source } \\
\text { Water }\end{array}$ & $\begin{array}{c}\text { Stream } \\
\#\end{array}$ & Note & Process Description & $\begin{array}{c}\text { Flow } \\
\text { (gpm) }\end{array}$ & Disposal Structure & $\begin{array}{l}\text { Well } \\
\text { Code }\end{array}$ & $\begin{array}{l}\text { Washington } \\
\text { State Planar } \\
\text { Coordinates } \\
\text { (meters) } \\
\end{array}$ & Comments & 216 & 218 \\
\hline $200 w$ & D & 133 & & $\begin{array}{l}\text { Steam Trap 2Q-Yard-MSS-TRP-027. This } \\
\text { steam trap discharges to the same injection } \\
\text { well as }-026 \text {. }\end{array}$ & $<1.00$ & Injection Well & $5 w_{20}$ & $\begin{array}{l}\text { I: } 567,522.01 \\
\text { N135,862.11 }\end{array}$ & $\begin{array}{l}\text { ELIMINATED 5/95. This stream is } \\
\text { a duplicate of Stream } 133 \text {. Steam line } \\
\text { from Powerthouse off of } 19 t h \text { street. } \\
\text { Front of trailer between powerhouse } \\
\text { and PFPP }\end{array}$ & No & No \\
\hline 20016 & $\mathrm{D}$ & 128 & & Sleaun Trap 2Q - Yard-MSS-TRP-028. & $<1.00$ & Injection Well & sw20 & $\begin{array}{l}\text { E } 567,339.32 \\
\text { N135,797.66 }\end{array}$ & $\begin{array}{l}\text { Steam line from Powerhouse beside } \\
\text { the road to T-Plant. 19th and } \\
\text { Bridgeport }\end{array}$ & No & Yes \\
\hline $200 \mathrm{w}$ & D & 129 & & Steam Trap 2Q - Yard-MSS-TRP-029. & $<1.00$ & Injection Well & $5 W 20$ & $\begin{array}{l}\text { ES67,125.98 } \\
\text { N135,797.17 }\end{array}$ & $\begin{array}{l}\text { Steam line from Powerhouse beside } \\
\text { the road to T-Plant. 19th and } \\
\text { Canadian Ave. }\end{array}$ & No & Yes \\
\hline $200 w$ & $\mathrm{D}$ & 130 & & Steam Trap 2Q-Yard-MSS-TRP-030. & $<1.00$ & Injection Well & $5 W 20$ & $\begin{array}{l}\text { ES67,004.11 } \\
\text { N135,796.92 }\end{array}$ & $\begin{array}{l}\text { Sleam line from Powerhouse beside } \\
\text { the road to T-Plant. Front of PFP } \\
\text { and } 19 \mathrm{th}\end{array}$ & No & Yes \\
\hline $200 \mathrm{~W}$ & $\mathrm{D}$ & 131 & & Steam Trap 2Q - Yard-MSS-TRP-031. & $<1.00$ & Injection Well & 5620 & $\begin{array}{l}\text { Es66,730.03 } \\
\text { N135,796.18 } \\
\end{array}$ & $\begin{array}{l}\text { Steam line from Powerhouse beside } \\
\text { the road to T-Plant. }\end{array}$ & No & Yes \\
\hline $200 \mathrm{~W}$ & D & 120 & & Steam Trap 2Q - Yard-MSS-TRP-037. & $<1.00$ & Injection Well & $5 W 20$ & $\begin{array}{l}E 567,397.70 \\
\text { N136,821.79 } \\
\end{array}$ & $\begin{array}{l}\text { Steam line from Powerhouse beside } \\
\text { the road to T.Plant. }\end{array}$ & No & Yes \\
\hline $200 \mathrm{~W}$ & D & 121 & & Steam Trap 2Q - Yard-MSS-TRP-038. & $<1.00$ & Injection Well & sw20 & $\begin{array}{l}\text { Es67,398.19 } \\
\text { N136,623.70 }\end{array}$ & $\begin{array}{l}\text { Steam line from Powerhouse beside } \\
\text { the road to T-Plant. }\end{array}$ & No & Yes \\
\hline $200 \mathrm{w}$ & D & 122 & & Steam Trap 2Q - Yard-MSS-TRP-039. & $<1.00$ & Injection Well & $5 W 20$ & $\begin{array}{l}\text { E567,398.50 } \\
\text { N1 } 36,495.70\end{array}$ & $\begin{array}{l}\text { Steam line from Powerhouse beside } \\
\text { the road to ' } \text {-Plant. }\end{array}$ & No & Yes \\
\hline $200 w$ & D & 123 & & Stean Trap $2 Q-Y$ ard-MSS-TRP-040. & $<1.00$ & Injection Well & $5 W 20$ & $\begin{array}{l}\text { E567,398.95 } \\
\text { N136,306.75 }\end{array}$ & $\begin{array}{l}\text { Steam line from Powerhouse beside } \\
\text { the road to T-Plant. }\end{array}$ & No & Yes \\
\hline $2001 \mathrm{~W}$ & D & 124 & & Steam Trap 2Q - Yard-MSS-TRP-04I. & $<1.00$ & Injection Well & $5 W 20$ & $\begin{array}{l}\text { ES67,399.14 } \\
\text { N136,227.52 }\end{array}$ & $\begin{array}{l}\text { Steam line from Powerhouse beside } \\
\text { the road to T-Plant. }\end{array}$ & No & Yes \\
\hline $200 \mathrm{w}$ & D & 125 & & Steam Trap 2Q - Yard-MSS-TRP-042, 043. & $<1.00$ & Injection Well & $5 w 20$ & $\begin{array}{l}\text { ES67,430.08 } \\
\text { N136,035.59 }\end{array}$ & $\begin{array}{l}\text { Steam line from Powerhouse beside } \\
\text { the road to 'T-Plant. }\end{array}$ & No & Yes \\
\hline Sourcen 11 & er ker: & A- & sundwate & B-Sufface Water & & D-Potzble Waler & & & & & \\
\hline
\end{tabular}


Table 3-1. Miscellaneous Streams Inventory

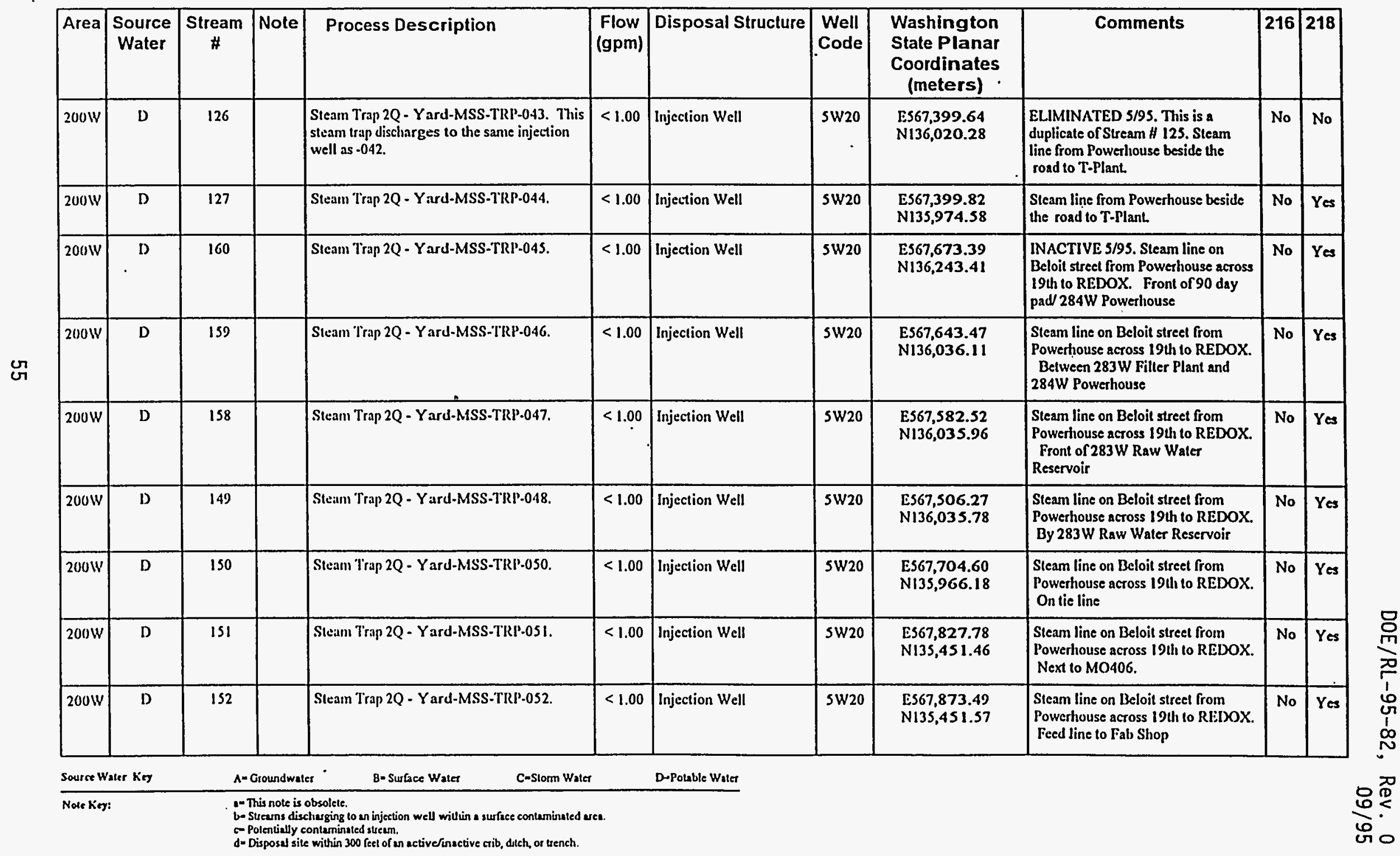


Table 3-1. Miscellaneous Streams Inventory

\begin{tabular}{|c|c|c|c|c|c|c|c|c|c|c|c|}
\hline Area & $\begin{array}{l}\text { Source } \\
\text { Water }\end{array}$ & $\begin{array}{c}\text { Stream } \\
\#\end{array}$ & Note & Process Description & $\begin{array}{c}\text { Flow } \\
\text { (gpm) }\end{array}$ & Disposal Structure & $\begin{array}{l}\text { Well } \\
\text { Code }\end{array}$ & $\begin{array}{c}\text { Washington } \\
\text { State Planar } \\
\text { Coordinates } \\
\text { (meters) } \\
\end{array}$ & Comments & 216 & 218 \\
\hline $200 \mathrm{~W}$ & D & 153 & & Steam Trap 2Q - Yard-MSS-TRP-0S3. & $<1.00$ & Injection Well & sw20 & $\begin{array}{l}\text { ES67,954.29 } \\
\text { N136,046.64 }\end{array}$ & $\begin{array}{l}\text { Steam line on Beloit street from } \\
\text { Powerhouse across } 19 \text { th to REDOX. } \\
\text { Ofr } 20 \text { th and Austin }\end{array}$ & No & Yes \\
\hline $200 \mathrm{~W}$ & D & 154 & & Steam Trap 2Q - Yard-MSS-TRP-054. & $<1.00$ & Injection Well & sW20 & $\begin{array}{l}\text { Es67,948.30 } \\
\text { N136,006.40 }\end{array}$ & \begin{tabular}{|l|} 
Steam line on Beloit street from \\
Powerhouse accoss 19 th to REDOX. \\
Front of $2707 \mathrm{~W}$ on 20 th
\end{tabular} & No & Yes \\
\hline $200 \mathrm{~W}$ & D & 157 & & Steam Trap 2Q - Yard-MSS-TRP-0SS. & $<1.00$ & Injection Well & $5 \mathrm{~W} 20$ & $\begin{array}{l}\text { ES67,948.22 } \\
\text { N } 136,036.87\end{array}$ & \begin{tabular}{|l|} 
Steam line on Beloit street from \\
Powerhouse across 19 th to REDOX. \\
Between HPT Office and 2707W
\end{tabular} & No & Yes \\
\hline $200 \mathrm{~W}$ & D) & iss & & Steam Tray 2Q - Yard-MSS-TRP'-056. & $<1.00$ & Injection Well & $5 \mathrm{~W} 20$ & $\begin{array}{l}\text { E567,948.11 } \\
\text { N } 136,079.54\end{array}$ & $\begin{array}{l}\text { Steam line on Beloit street from } \\
\text { Powerhouse across } 19 \text { th to REDOX. } \\
\text { Between } 277 \text { W and HPT Office }\end{array}$ & No & Yes \\
\hline $200 \mathrm{~W}$ & D & 156 & & Sleam Trap 2Q - Yard-MSS-TRP-057. & $<1.00$ & Injection Well & $5 W_{20}$ & $\begin{array}{l}\text { ES667,978.57 } \\
\text { N136,085.71 }\end{array}$ & $\begin{array}{l}\text { Steam line on Beloit street from } \\
\text { Powerhouse across } 19 \text { th to REDOX. } \\
\text { Comer of } 2707 \mathrm{~W} \text { and Machine } \\
\text { Shop }\end{array}$ & No & Yes \\
\hline $2001 \mathrm{w}$ & D & 646 & & $\begin{array}{l}\text { Sleam Trap 2Q-Yard-MSS-TRP-058 - } \\
\text { Steam condensale. }\end{array}$ & $<0.01$ & Injection well & $5 W 20$ & $\begin{array}{l}\text { ES67,978.48 } \\
\text { N136,124.41 }\end{array}$ & $\begin{array}{l}\text { CORRECTED: 7/6/95 per CC;Mail } \\
\text { from M. Gunter dated 6/29/95. } \\
\text { ADDED 6/95. On line } 805 \text { to the } \\
\text { water tower. }\end{array}$ & No & Yes \\
\hline $200 \mathrm{~W}$ & D & 647 & & $\begin{array}{l}\text { Steaun Trap 2Q-Yard-MSS-IRP-059- } \\
\text { Steam condensule. }\end{array}$ & $<0.01$ & Injection well & $5 W 20$ & $\begin{array}{l}\text { Es67,932.95 } \\
\text { N136,052.07 }\end{array}$ & $\begin{array}{l}\text { ADDED 6/25. On line } 803 \text { to } \\
2723 w\end{array}$ & No & Yes \\
\hline $2001 \mathrm{~W}$ & D & 648 & & $\begin{array}{l}\text { Sleam Trap } 2 Q \cdot Y \text { ard-MISS-TRP-060 - } \\
\text { Steam condensite. }\end{array}$ & $<0.01$ & Injection well & $5 W 20$ & $\begin{array}{l}\text { ES67,735.05 } \\
\text { N } 135,975.40\end{array}$ & $\begin{array}{l}\text { ADDED 6/95. On line } 803 \text { to } \\
\text { 2719WA. }\end{array}$ & No & Yes \\
\hline $200 \mathrm{~W}$ & D & 649 & & $\begin{array}{l}\text { Steam Trap 2Q-Yard-MSS-TRR-061- } \\
\text { Steam condensale. }\end{array}$ & $<0.01$ & Injection well & $5 W_{20}$ & $\begin{array}{l}\text { Es67,917.78 } \\
\text { N136,021.56 }\end{array}$ & $\begin{array}{l}\text { ADDED 6/95. On line } 806 \text { to } \\
\text { 2707W }\end{array}$ & No & Yes \\
\hline $200 \mathrm{w}$ & D & 650 & & $\begin{array}{l}\text { Steam Trap 2Q-Yard-MSS-TRP-062 - } \\
\text { Stean condensate. }\end{array}$ & $<0.01$ & Injection well & $5 w_{20}$ & $\begin{array}{l}\text { E:567,917.71 } \\
\text { N136,052.04 }\end{array}$ & $\begin{array}{l}\text { CORRECTED: } 7 / 6 / 95 \text { per CC:Mail } \\
\text { from M. Gunter dated } 6 / 29 / 95 \text {. } \\
\text { ADDFD } 6 / 95 \text {. On line } 805 \text { to } 272 \text { W. }\end{array}$ & No & Yes \\
\hline Source & Iateri Key & $\hat{A}$ & roundwatc & B- Surface Waler & & D-Potable Wata & & & & & \\
\hline
\end{tabular}


Table 3-1. Miscellaneous Streams Inventory

\begin{tabular}{|c|c|c|c|c|c|c|c|c|c|c|c|}
\hline Area & \begin{tabular}{|l|} 
Source \\
Water
\end{tabular} & $\begin{array}{c}\text { Stream } \\
\#\end{array}$ & Note & Process Description & \begin{tabular}{|l|} 
Flow \\
(gpm)
\end{tabular} & Disposal Structure & \begin{tabular}{c|} 
Well \\
Code
\end{tabular} & $\begin{array}{l}\text { Washington } \\
\text { State Planar } \\
\text { Coordinates } \\
\text { (meters) }\end{array}$ & Comments & 216 & 218 \\
\hline $200 w$ & D & 261 & b & $\begin{array}{l}\text { Steant Turbine - Condensale discharged to } \\
\text { frencli drain } 216-Z-13 \text {. }\end{array}$ & 0.50 & Injection Well & sw20 & $\begin{array}{l}\text { ES66,498.13 } \\
\text { N135,582.02 }\end{array}$ & $\begin{array}{l}\text { Potential historical contamination } \\
\text { underground at discharge location. }\end{array}$ & No & Yes \\
\hline $200 \mathrm{~W}$ & D & 262 & b & $\begin{array}{l}\text { Steam Turline - Condensale discharged to } \\
\text { french drain 216-2-14. }\end{array}$ & 0.50 & Injection Well & $5 W_{20}$ & $\begin{array}{l}\text { ES66,479.73 } \\
\text { N135,625.26 }\end{array}$ & $\begin{array}{l}\text { Potential historical contamination } \\
\text { underground at discharge location. }\end{array}$ & No & Yes \\
\hline $200 w$ & D & 640 & & $\begin{array}{l}\text { W-IS Sheet Metal Shop - Kaiser } \\
\text { Consinuction Yard - IIVAC condensale. }\end{array}$ & 0.05 & Injection well & $5 W 20$ & $\begin{array}{l}\text { ES67,288.00 } \\
\text { N13S,910.00 }\end{array}$ & $\begin{array}{l}\text { ADDED 6/95. North side of the sheet } \\
\text { metal shop in the KII cosntruction } \\
\text { yard. Possible duplicate of } \$ 46 \text {. }\end{array}$ & No & Yes \\
\hline $2001 \mathrm{~V}$ & D) & 46 & & W-15 Sheet Metal Shop - Steam conderisate. & 0.05 & Injection Well & $5 W 20$ & $\begin{array}{l}\text { ES67,288.90 } \\
\text { N135,910.66 }\end{array}$ & CORRECTED 2/95. Contact added. & No & Yes \\
\hline $200 \mathrm{w}$ & D & 40 & & W-18 Insulator's Shop - Steam condensnte. & 0.05 & Injection Well & $5 W_{20}$ & $\begin{array}{l}\text { ES67,260.17 } \\
\text { N135,829.82 }\end{array}$ & $\begin{array}{l}\text { CORRECTED 2/95. Contact added. } \\
\text { South }\end{array}$ & No & Yes \\
\hline $200 \mathrm{w}$ & D & 41 & & W-18 hisulator's Shop - Steam condensate. & 0.05 & Injection Well & $5 \mathrm{~W} 20$ & $\begin{array}{l}\text { ES } 67,266.00 \\
\text { N135,829.80 }\end{array}$ & CORRECTED 2/95. Conlact added. & No & Yes \\
\hline $200 \mathrm{w}$ & D & 43 & & W-20 Piperitter's Shop - Steam condensate. & 0.05 & Injection Well & $5 W 20$ & $\begin{array}{l}\text { ES67,245.58 } \\
\text { N135,862.94 }\end{array}$ & |CORRECTED 2/95. Conlact added. & No & Yes \\
\hline $200 \mathrm{w}$ & D & 638 & & $\begin{array}{l}\text { W.27 Pipefitter's Shop - Kaiser Constniction } \\
\text { Yard - Steam condensate. }\end{array}$ & 0.05 & Injection well & $5 W_{20}$ & $\begin{array}{l}\text { E.S67,228.00 } \\
\text { N } 135,828.00\end{array}$ & $\begin{array}{l}\text { ADDED 6/95. Within Kaiser } \\
\text { Construction Yard. On south end of } \\
\text { building, west side. }\end{array}$ & No & Yes \\
\hline $200 \mathrm{w}$ & D & 639 & & $\begin{array}{l}\text { W-27 Pipefilter's Shop - Kaiser Construction } \\
\text { Yard - Steam condensate. }\end{array}$ & 0.05 & Injection well & $5 W_{20}$ & $\begin{array}{l}\text { ES } 567,228.00 \\
\text { N135,835.00 }\end{array}$ & $\begin{array}{l}\text { ADDED 6/95. Within Kaiser } \\
\text { Construction Yard. On north end of } \\
\text { building, west side. Possible } \\
\text { duplicale of } \$ 38 \text {. }\end{array}$ & No & Yes \\
\hline $200 \mathrm{w}$ & D & 38 & & $\begin{array}{l}\text { W-27 Piperfitter's Shop and W-26 Carpenter's } \\
\text { Sliop - Steam condensate. }\end{array}$ & 0.01 & Injection Wall & sw20 & $\begin{array}{l}\text { ES } 567,235.32 \\
\text { N135,837.10 } \\
\end{array}$ & CORRISCTED 2/95. Contact added. & No & Yes \\
\hline $200 \mathrm{w}$ & D & 407 & & $\begin{array}{l}\text { WRAP I Building mechanical room. } \\
\text { Compressor Condensale and IIVAC } \\
\text { condensate. Due to the oil blow associated } \\
\text { with condensantion, an oil-water seperator } \\
\text { will be installed in the discharge line. }\end{array}$ & $<0.01$ & Gravel Basin & N/A & $\begin{array}{l}\text { E.565,839.59 } \\
\text { N136,552.79 }\end{array}$ & $\begin{array}{l}\text { ADDED 12/94. Scheduled to be } \\
\text { added 3/96. Gravel basins are to be } \\
\text { constructed wider than deep. North of } \\
\text { the mechanical room. }\end{array}$ & Yes & No \\
\hline
\end{tabular}


Table 3-1. Miscellaneous Streams Inventory

\begin{tabular}{|c|c|c|c|c|c|c|c|c|c|c|c|}
\hline Area & $\begin{array}{l}\text { Source } \\
\text { Water }\end{array}$ & $\begin{array}{c}\text { Stream } \\
\#\end{array}$ & Note & Process Description & $\begin{array}{c}\text { Flow } \\
\text { (gpm) }\end{array}$ & Disposal Structure & $\begin{array}{l}\text { Well } \\
\text { Code }\end{array}$ & $\begin{array}{l}\text { Washington } \\
\text { State Planar } \\
\text { Coordinates } \\
\text { (meters) } \\
\end{array}$ & Comments & 216 & 218 \\
\hline $2001 \mathrm{~W}$ & D & 424 & & $\begin{array}{l}\text { WRAP' I facility Truepact Unload Bay } \\
\text { Drain. Trucks will be washed with a high } \\
\text { pressure hose to remove the buildup of snow } \\
\text { and ice on the vehicles. }\end{array}$ & $<0.01$ & Gravel Basins & $N / A$ & $\begin{array}{l}\text { E566,916.97 } \\
\text { N136,511.31 }\end{array}$ & $\begin{array}{l}\text { ADDED 12/94. Scheduled to be } \\
\text { added 3/96. Gravel basins are to be } \\
\text { constructed wider than deep. The } \\
\text { Basins are located east of the WRAP } \\
1 \text { facility }\end{array}$ & Yes & No \\
\hline 300 & c & $\$ 24$ & & $\begin{array}{l}300 \text { Area south parking lot Stonnwater } \\
\text { Collection System. }\end{array}$ & 5.00 & Collection Basin & N/A & $\begin{array}{l}E 593,821.47 \\
\text { N115,569.97 }\end{array}$ & ADDED 2/95. East of 3790. & Yes & No \\
\hline 300 & D) & 495 & & $\begin{array}{l}\text { 303C Building - Steam condensate from } \\
\text { main header, UPD-TRP'007,008. }\end{array}$ & $<0.01$ & Injection Well & SW20 & $\begin{array}{l}\text { E593,852.00 } \\
\text { N116,075.00 }\end{array}$ & $\begin{array}{l}\text { ADDED 10/94. 303C Northeast } \\
\text { corner }\end{array}$ & No & Yes \\
\hline 300 & D) & 352 & & $\begin{array}{l}303 \mathrm{~F} \text { 13uilding stean condensate, was fed by } \\
\text { line from } 313 \text { building. This line is no longer } \\
\text { in service. }\end{array}$ & 0.00 & Covered ditch, not UIC. & N/A & $\begin{array}{l}\text { E.593,883.36 } \\
\text { N } 116,106.17\end{array}$ & $\begin{array}{l}\text { ELIMINATED 10/94. West side } \\
\text { 303F. Scheduled to be removed. }\end{array}$ & No & No \\
\hline 300 & $D$ & 267 & & 303 J Building - IIVAC condensate. & $<0.01$ & Injection Well & $5 W 20$ & $\begin{array}{l}E 593,979.07 \\
\text { N116,074.44 } \\
\end{array}$ & $303 \mathrm{~J}$ - north side of building & No & Yes \\
\hline 300 & D & 266 & & $\begin{array}{l}303 \mathrm{~J} \text { lBuilding - Steam condensate mud leg. } \\
\text { (part of } 300 \text { main supply). }\end{array}$ & $<0.50$ & Injection Well & $5 W 20$ & $\begin{array}{l}\text { E593,979.42 } \\
\text { N116,050.82 }\end{array}$ & $303 \mathrm{~J}$ - south side of building & No & Yes \\
\hline 300 & $\mathrm{D}$ & 415 & & 305 Building - Steam condensalc. & $<0.01$ & Injection Well & $5 W 20$ & $\begin{array}{l}\text { E593,771.95 } \\
\text { N116,214.36 }\end{array}$ & $\begin{array}{l}\text { ADDED 10/94. Northeast comer of } \\
\text { 305. }\end{array}$ & No & Yes \\
\hline 300 & D & 416 & & 305 Building - Steam condensate. & $<0.01$ & Injection Well & $5 W 20$ & $\begin{array}{l}E 593,763.23 \\
\text { N116,185.59 }\end{array}$ & $\begin{array}{l}\text { ADDED 10/94. South side of } 305 \\
12 \text { to } 15 \text { feet west of roll up door, } 4 \\
\text { feet from edge of the building. }\end{array}$ & No & Yes \\
\hline 300 & D & 417 & & 305 Building - Sleam condensate. & $<0.01$ & Injection Well & $5 W 20$ & $\begin{array}{l}\text { ES93,722.00 } \\
\text { N116,191.00 }\end{array}$ & $\begin{array}{l}\text { ADDED 10/94. Soutliwest comer of } \\
305 .\end{array}$ & No & Yes: \\
\hline 300 & D & 451 & & 305 Building - Steam condensate. & $<0.01$ & Injection Well & SW20 & $\begin{array}{l}\text { E:593,738.00 } \\
\text { N116,189.00 }\end{array}$ & $\begin{array}{l}\text { NDOLD 2/95. Main steam line } \\
\text { south side of } 305 \text { building by } \\
\text { entrance. }\end{array}$ & No & Yes \\
\hline 300 & $\mathrm{D}$ & 449 & & 305B Building - Steam condensate. & 0.00 & Injection Well & $5 W 20$ & $\begin{array}{l}\text { E593,706.34 } \\
\text { N116,146.65 }\end{array}$ & ADDED 10/94. Soulliwes! 305B. & No & Yes \\
\hline
\end{tabular}


Table 3-1. Miscellaneous Streams Inventory

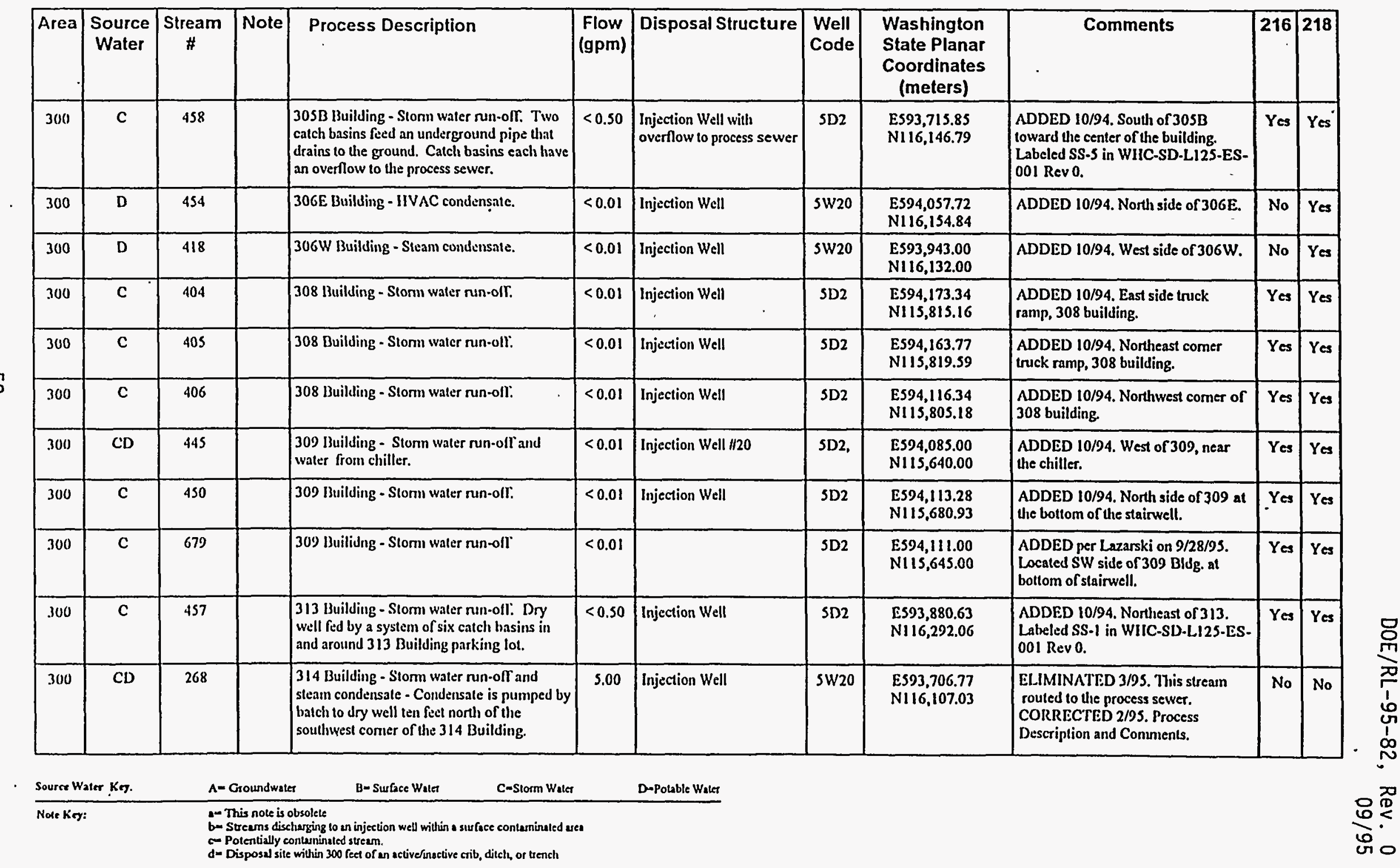


Table 3-1. Miscellaneous Streams Inventory

\begin{tabular}{|c|c|c|c|c|c|c|c|c|c|c|c|}
\hline Area & $\begin{array}{l}\text { Source } \\
\text { Water }\end{array}$ & $\begin{array}{c}\text { Stream } \\
\#\end{array}$ & Note & Process Description & $\begin{array}{l}\text { Flow } \\
\text { (gpm) }\end{array}$ & Disposal Structure & $\begin{array}{l}\text { Well } \\
\text { Code }\end{array}$ & $\begin{array}{c}\text { Washington } \\
\text { State Planar } \\
\text { Coordinates } \\
\text { (meters) }\end{array}$ & Comments & 216 & 218 \\
\hline 300 & $A$ & 350 & & 320 Building - & $<0.10$ & Injection Well & SF1 & $\begin{array}{l}E 593,768.26 \\
\text { N115,509.36 }\end{array}$ & $\begin{array}{l}\text { ELIMINATED per Lazarski on } \\
\text { 9/28/95. Northwest of } 320 \text { Building }\end{array}$ & No & No \\
\hline 300 & $A$ & 626 & & $\begin{array}{l}320 \text { Building - French drain receives eflluent } \\
\text { from irrigation lines when lines are } \\
\text { evacuated during the fall. }\end{array}$ & $<0.10$ & Injection Well & $5 \mathrm{Fl}$ & $\begin{array}{l}E S 93,760.00 \\
N 115,530.00\end{array}$ & $\begin{array}{l}\text { ADDED 4/95. North of } 320 \\
\text { Building }\end{array}$ & Yes & Yes \\
\hline 300 & $A$ & 627 & & $\begin{array}{l}320 \text { Building - French drain receives eflluent } \\
\text { from irrigation lines when lines are } \\
\text { evacuated during the fall. }\end{array}$ & $<0.10$ & Injection Well & SFI & $\begin{array}{l}\text { ES93,815.00 } \\
\text { N } 115,532.00\end{array}$ & $\begin{array}{l}\text { ADDED 4/95. Northeast of } 320 \\
\text { Building }\end{array}$ & Yes & Yes \\
\hline 300 & $\Lambda$ & 628 & & $\begin{array}{l}320 \text { Building - French drain receives eflluent } \\
\text { from irrigation lines when lines are } \\
\text { evacualed during the fall. }\end{array}$ & $<0.10$ & $\begin{array}{l}\text { Northeast of } 320 \\
\text { Building }\end{array}$ & sin & $\begin{array}{l}\text { ES93,860.00 } \\
\text { N115,510.00 }\end{array}$ & $\begin{array}{l}\text { ADDED 4/95. Northeast of } 320 \\
\text { Building }\end{array}$ & Yes & Yes \\
\hline 300 & D & 370 & & 321 Building - Steam condensate. & $<0.01$ & Injection Well & $5 W 20$ & $\begin{array}{l}E 593,777.37 \\
\text { N115,875.96 }\end{array}$ & West side 321 building & No & Yes \\
\hline 300 & D & 371 & & 321 Building - Steam condensate. & $<0.01$ & Injection Well & $5 W 20$ & $\begin{array}{l}\text { ES93,772.68 } \\
\text { N115,852.42 } \\
\end{array}$ & West side 321 building & No & Yes \\
\hline 300 & D & 372 & & 321 Building - Steam condensate. & $<0.01$ & $\begin{array}{l}\text { Injection Well, Labelled } \\
\text { as F.D. H35 }\end{array}$ & sw20 & $\begin{array}{l}E 593,800.00 \\
\text { N115,859.00 }\end{array}$ & $\begin{array}{l}\text { Bottom of the truck ramp on the } \\
\text { south side of } 321 \text { building. }\end{array}$ & No & Yes \\
\hline 300 & D & 348 & & 321 Building - Vent valve on water line. & $<0.01$ & Injection Well & $5 W 20$ & $\begin{array}{l}E 593,769.59 \\
\text { N115,855.42 }\end{array}$ & 321 Building, West side & Yes & Yes \\
\hline 300 & $\mathbf{C}$ & 680 & & 321 Building - storn water run-ofr & $<0.01$ & Injection Well & SD2 & $\begin{array}{l}E 593,807.00 \\
\text { N115,862.00 } \\
\end{array}$ & ADDED $9 / 28 / 95$ & Yes & Yes \\
\hline 300 & $\mathrm{D}$ & 453 & & 323 Building - Steam condensate. & $<0.01$ & Injection Well & SD2 & $\begin{array}{l}E S 93,782.92 \\
N 115,809.44 \\
\end{array}$ & $\begin{array}{l}\text { ADDED 10/94. South side of } 323 \\
\text { near the west comer. }\end{array}$ & No & Yes \\
\hline 300 & $\mathrm{C}$ & 354 & & 324 Building - Stonn water run-oll. & $<0.05$ & Injection Well & SD2 & $\begin{array}{l}\text { ES94,217.05 } \\
\text { N115,744.56 }\end{array}$ & $\begin{array}{l}\text { ELIMINATED 3/95. This stream } \\
\text { routed to the process sewer. ADDED } \\
10 / 94 \text {. The stream in the original } \\
\text { inventory was the same as } 353 \text {. This } \\
\text { stream has been added in its place. } \\
\text { South of building } 324\end{array}$ & No & No \\
\hline
\end{tabular}

B- Surface Walet

C-Storm Water DePolable Water 
Table 3-1. Miscellaneous Streams Inventory

\begin{tabular}{|c|c|c|c|c|c|c|c|c|c|c|c|}
\hline Area & $\begin{array}{l}\text { Source } \\
\text { Water }\end{array}$ & $\begin{array}{c}\text { Stream } \\
\#\end{array}$ & Note & Process Description & $\begin{array}{c}\text { Flow } \\
\text { (gpm) }\end{array}$ & Disposal Structure & $\begin{array}{l}\text { Well } \\
\text { Code }\end{array}$ & $\begin{array}{l}\text { Washington } \\
\text { State Planar } \\
\text { Coordinates } \\
\text { (meters) }\end{array}$ & Comments & 216 & 218 \\
\hline 300 & $\mathrm{CD}$ & 425 & & $\begin{array}{l}324 / 336 \text { Building - Stomm water run-ofr and } \\
\text { steam condensate. }\end{array}$ & $<0.05$ & Injection Well & SD2 & $\begin{array}{l}\text { E594,396.00 } \\
\text { NIIS,729.00 }\end{array}$ & $\begin{array}{l}\text { ADDED steam condensate as a } \\
\text { stream source per Lazarski } 9 / 28 / 95 \text {. } \\
\text { ADDED } 10 / 94 \text {. Southeast of } 336 \text {. } \\
324 \text { Bldg. emuent piped to this } \\
\text { location }\end{array}$ & Yes & Yes \\
\hline 300 & c & 264 & & $\begin{array}{l}325 \text { Building - Rain water from leaky roof. } \\
\text {. }\end{array}$ & $<0.01$ & Injection Well & SD2 & $\begin{array}{l}\text { ES93,978.00 } \\
\text { N115,745.00 }\end{array}$ & $\begin{array}{l}\text { CORRECTED 2/95. Process } \\
\text { Description and Comments. Inside } \\
325 \text { Building, south side stairwell. } \\
\text { Accessed via cafeteria. }\end{array}$ & Yes & $\overline{\text { Yes }}$ \\
\hline 300 & D & 265 & & $\begin{array}{l}325 \text { Building - Steim condensale; discharged } \\
\text { to dry well. }\end{array}$ & $<0.50$ & Injection Well No. 399-3 & $5 W 20$ & $\begin{array}{l}\text { ES94,023.00 } \\
\text { N115,828.00 }\end{array}$ & $\begin{array}{l}\text { CORRECTED 2/95. Coordinates. } \\
\text { Northeast comer of } 325 \text {, located } \\
\text { beneath clevated conpressed gas } \\
\text { storage dock. }\end{array}$ & No & Yes \\
\hline 300 & $\mathrm{CD}$ & 408 & & 325 Building Stormwaler. & $<0.01$ & Chiller Pad & $\mathrm{N} / \mathrm{A}$ & $\begin{array}{l}\text { Es94,045.05 } \\
\text { N115,808.71 }\end{array}$ & $\begin{array}{l}\text { ELIMINATED 3/95. ADDED } \\
\text { 10/94. East of building } 325 \text { on chiller } \\
\text { pad. }\end{array}$ & No & No \\
\hline 300 & $\mathrm{CD}$ & 409 & & $\begin{array}{l}326 \text { Building - Storm water run-off and } \\
\text { steatil condensate. }\end{array}$ & $<0.01$ & Injection Well & 5D2 & $\begin{array}{l}\text { ES93,934.65 } \\
\text { N115,854.73 }\end{array}$ & $\begin{array}{l}\text { ELIMINATED 3/95. This stream } \\
\text { routed to the sanitary sewer. } \\
\text { CORRECTED 2/95. Process } \\
\text { Description. ADDED 10/94. } \\
\text { Southeast of } 326 .\end{array}$ & No & No \\
\hline 300 & D & 353 & & $\begin{array}{l}328 \text { Building - French drain; stean } \\
\text { condensate. }\end{array}$ & $<0.01$ & Injection Well & $5 W 20$ & $\begin{array}{l}\text { ES94,019.89 } \\
\text { N115,889.11 }\end{array}$ & $\begin{array}{l}328 \text { Building, North side of NE } \\
\text { Annex }\end{array}$ & Yes & Yes \\
\hline 300 & c & 422 & & 329 [Building - Slomi water run-olf. & $<0.01$ & Injection Well & SD2 & $\begin{array}{l}\text { ES93,917.14 } \\
\text { NI15,749.68 }\end{array}$ & $\begin{array}{l}\text { ELIMINATED 3/95. This stream } \\
\text { routed to the process sewer. ADDED } \\
\text { 10/94. East side of } 329 \text { near the } \\
\text { south side. }\end{array}$ & No & No \\
\hline 300 & $\mathrm{C}$ & 546 & & 329 Building - Storn walter run-oll. & $<1.00$ & Injection Well & SD2 & $\begin{array}{l}\text { ES93,916.86 } \\
\text { N115,769.03 }\end{array}$ & $\begin{array}{l}\text { ELIMINATLD 3/95. This stream } \\
\text { routed to the process sewer. ADDED } \\
2 / 95 \text {. East side soull of center }\end{array}$ & No & $\overline{\text { No }}$ \\
\hline
\end{tabular}

\begin{tabular}{|c|c|c|}
\hline Source Water Kegr. & B-Sufface Water & C-Storm Water \\
\hline
\end{tabular}


Table 3-1. Miscellaneous Streams Inventory

\begin{tabular}{|c|c|c|c|c|c|c|c|c|c|c|c|}
\hline Area & $\begin{array}{l}\text { Source } \\
\text { Water }\end{array}$ & $\begin{array}{c}\text { Stream } \\
\#\end{array}$ & Note & Process Description & $\begin{array}{l}\text { Flow } \\
\text { (gpm) }\end{array}$ & Disposal Structure & $\begin{array}{l}\text { Well } \\
\text { Code }\end{array}$ & $\begin{array}{c}\text { Washington } \\
\text { State Planar } \\
\text { Coordinates } \\
\text { (meters) }\end{array}$ & Comments & 216 & 218 \\
\hline 300 & & 574 & & 331 Building - Stean condensate. & 0.00 & & & $\begin{array}{l}\text { Es94,471.01 } \\
\text { N115,403.31 }\end{array}$ & $\begin{array}{l}\text { ELIMINATED not an injection well } \\
\text { per Lazarski } 9 / 28 / 95 \text {. ADDED } 3 / 95 \text {. } \\
\text { West side of } 331 \text {, south of } 331-A \text {. }\end{array}$ & No & No \\
\hline 300 & D) & $\$ 13$ & & 331 Building - Sleam condensate. & $<1.00$ & Injection Well & sw20 & $\begin{array}{l}\text { E594,497.41 } \\
\text { N115,462.89 }\end{array}$ & $\begin{array}{l}\text { ADDED 2/95. } 331 \text { Building } 10 \text { feet } \\
\text { north of the northwest comer. }\end{array}$ & No & Yes \\
\hline 300 & c & 447 & & 331 Building - Storm water nun-ofl: & $<0.01$ & Injection Well $\# 32$ & SD2 & $\begin{array}{l}\text { E594,469.00 } \\
\text { N115,383.00 }\end{array}$ & $\begin{array}{l}\text { ADDED 10/94. West side of } 331 \text { by } \\
\text { kenuels. }\end{array}$ & Yes & Yes \\
\hline 300 & c & 4.48 & & 331 Building - Storm waler nun-olr. & $<0.01$ & Irjection Well H37 & 5D2 & $\begin{array}{l}\text { ES94,492.98 } \\
\text { N115,453.32 }\end{array}$ & $\begin{array}{l}\text { ADDED 10/94. West side } 30 \text { from } \\
\text { the nortliwest comes of } 331 \text {. }\end{array}$ & Yes & Yes \\
\hline 300 & c & 456 & & 333 Building - Stom watler nuth-ofr. & $<0.50$ & $\begin{array}{l}\text { Injection Well with an } \\
\text { overflow to process sewer }\end{array}$ & SD2 & $\begin{array}{l}\text { E593,996.58 } \\
\text { N116,179.49 }\end{array}$ & $\begin{array}{l}\text { ADDED 10/94. East side of } 333 \text { near } \\
\text { the south end. Labeled SS-3 in } \\
\text { WIIC-SD-LL125-ES-001 Rev } 0 .\end{array}$ & Yes & Yes \\
\hline 300 & c & 455 & & $\begin{array}{l}333 \text { Building - Storm water nun-oif. Drywell } \\
\text { is below grade, draining a network of four } \\
\text { catch basins. Drywell is approximately } 60 \\
\text { feet north of } 333 \text { Building. }\end{array}$ & $<0.50$ & Injection Well & SD2 & $\begin{array}{l}\text { E593,966.33 } \\
\text { N } 116,293.32\end{array}$ & $\begin{array}{l}\text { ADDED 10/94. } 60 \text { feet north of } 333 \text {, } \\
\text { labeled SS-2 in WHIC-SD-Li25-ES- } \\
\text { 00I Rev } 0 .\end{array}$ & Yes & Yes \\
\hline 300 & $\mathrm{D}$ & 428 & & 340 lsuilding - (P-3 pump pit) pump leaks. & $<0.01$ & Injection Well & SA19 & $\begin{array}{l}\text { Es94,174.96 } \\
\text { N115,898.46 }\end{array}$ & $\begin{array}{l}\text { ADDED 10/94. Bottom of P-3 pump } \\
\text { pit. }\end{array}$ & Yes & Yes \\
\hline 300 & $\mathrm{D}$ & 341 & & $\begin{array}{l}340 \text { Building - Steam condensale and } \\
\text { cooling water. }\end{array}$ & $<0.01$ & Injection Well & $5 w 20$ & $\begin{array}{l}\text { Es94,149.53 } \\
\text { N115,917.37 } \\
\end{array}$ & 340 Building, Southwest comer & Yes & Yes \\
\hline 300 & $D$ & 427 & & 340 A Building - Steam condensate. & $<0.01$ & Injection Well 1143 & $5 W 20$ & $\begin{array}{l}E 594,207.04 \\
\text { N115,938.73 } \\
\end{array}$ & ADDED 10/94. East side of $340 \mathrm{~A}$ & No & Yes \\
\hline 300 & D & 426 & & 34013 Building - Ilose flusli water. & $<0.01$ & Injection Well & $5 W 20$ & $\begin{array}{l}\text { E.594,171.59 } \\
\text { N115,951.53 } \\
\end{array}$ & ADDED 10/94. East side of $340 \mathrm{~B}$. & Yes & Yes \\
\hline .300 & D & 381 & & 3506A Building - Steam condensale. & $<0.01$ & Injection Well & sw20 & $\begin{array}{l}\text { ES93,847.11 } \\
\text { N115,977.25 } \\
\end{array}$ & North center $3506 \mathrm{~A}$ & No & Yes \\
\hline 300 & $\mathrm{v}$ & 382 & & 3506A Building - Steam condensate. & $<0.01$ & Injection Weli & $5 W 20$ & $\begin{array}{l}\text { ES93,856.62 } \\
\text { N115,977.39 }\end{array}$ & Northeast comer $3506 \wedge$ Building & No & Yes \\
\hline
\end{tabular}

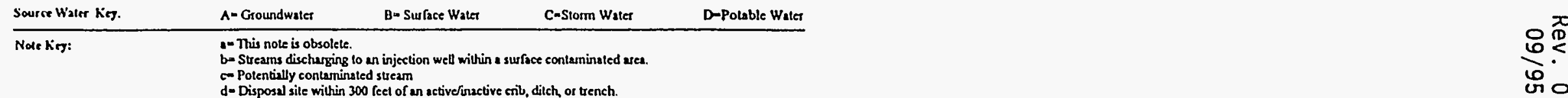


Table 3-1. Miscellaneous Streams Inventory

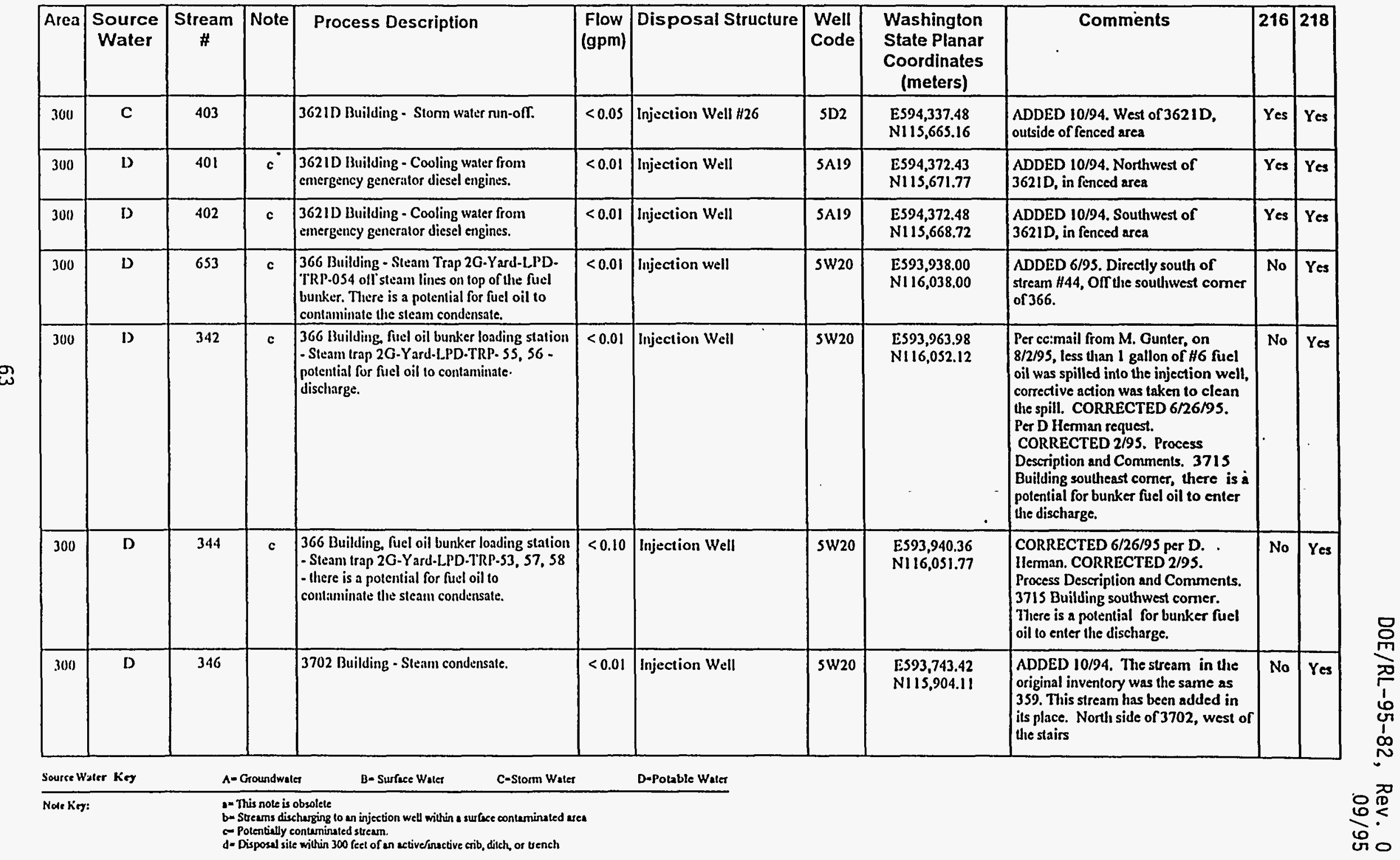


Table 3-1. Miscellaneous Streams Inventory

\begin{tabular}{|c|c|c|c|c|c|c|c|c|c|c|c|}
\hline Area & $\begin{array}{l}\text { Source } \\
\text { Water }\end{array}$ & $\begin{array}{c}\text { Stream } \\
\#\end{array}$ & Note & Process Description & $\begin{array}{c}\text { Flow } \\
\text { (gpm) }\end{array}$ & Disposal Structure & $\begin{array}{l}\text { Well } \\
\text { Code }\end{array}$ & $\begin{array}{l}\text { Washington } \\
\text { State Planar } \\
\text { Coordinates } \\
\text { (meters) }\end{array}$ & Comments & 216 & 218 \\
\hline 300 & $\mathrm{D}$ & 431 & & 3703 Building - Steam condensate. & $<0.01$ & Injection Well H9 & $5 W 20$ & $\begin{array}{l}\text { Es93,690.22 } \\
\text { N116,284.34 } \\
\end{array}$ & ADDED 10/94. South center 3703. & No & Yes \\
\hline 300 & $\mathrm{C}$ & 410 & & 370s Building - Storm water nun-olr. & $<0.01$ & Injection Well & SD2 & $\begin{array}{l}E 593,712.03 \\
\text { N116,070.53 }\end{array}$ & $\begin{array}{l}\text { ADDED 10/94. Northeast corner of } \\
3705\end{array}$ & Yes & Yes \\
\hline 300 & $\mathrm{c}$ & 411 & & 370 S Building - Stom waler nun-ofr. & $<0.01$ & Injection Well & SD2 & $\begin{array}{l}\text { E593,688.41 } \\
\text { N116,070.19 }\end{array}$ & $\begin{array}{l}\text { ADDED 10/94. Northwest comer of } \\
3705 \text {. }\end{array}$ & Yes & Yes \\
\hline 300 & C & 412 & & 3705 Building - Stom water run-oft. & $<0.01$ & Injection Well & SD2 & $\begin{array}{l}E 593,712.45 \\
\text { N116,041.98 }\end{array}$ & $\begin{array}{l}\text { ADDED 10/94. Southeast comer of } \\
3705 \text {. }\end{array}$ & Yes & Yes \\
\hline 300 & c & 413 & & 3705 Btuilding - Storm water null-otr. & $<0.01$ & Injection Well & SD2 & $\begin{array}{l}\text { E593,650.00 } \\
\text { N116,051.00 }\end{array}$ & $\begin{array}{l}\text { ADDED 10/94. Southwest comer of } \\
3705 \text {. }\end{array}$ & Yes & Yes \\
\hline 300 & $\mathrm{CD}$ & 515 & & $\begin{array}{l}3706 \text { Building - Discharge point for lire } \\
\text { sprinkler system water, consisting of a half } \\
\text { inch relief line and a two inch main line. }\end{array}$ & $<1.00$ & Injection Well & SD2, & $\begin{array}{l}\text { ES93,761.42 } \\
\text { NIIS,976.00 }\end{array}$ & $\begin{array}{l}\text { ADDED 2/95. } 3706 \text { Building center } \\
\text { of north side. }\end{array}$ & No & Yes \\
\hline 300 & $\mathrm{D}$ & 356 & & 3706 Building - Steam condensate. & $<0.05$ & $\begin{array}{l}\text { Injection Well with } \\
\text { overflow to process } \\
\text { sewer. }\end{array}$ & $5 W 20$ & $\begin{array}{l}\text { E593,809.05 } \\
\text { N115,976.70 }\end{array}$ & Northeast comer of 3706 & No & Yes \\
\hline 300 & D) & 357 & & 3706 Building - Steam condensate. & $<0.05$ & $\begin{array}{l}\text { linjection Well willı } \\
\text { overflow to process } \\
\text { sewer. }\end{array}$ & $5 W 20$ & $\begin{array}{l}\text { E593,752.16 } \\
\text { Ni15,956.82 }\end{array}$ & $\begin{array}{l}\text { North-central portion of } 3706 \text { main } \\
\text { courtyard }\end{array}$ & No & Yes \\
\hline 300 & $\mathrm{D}$ & 358 & & 3706 Building - Steam condensnte. & $<0.05$ & $\begin{array}{l}\text { Injection Well with } \\
\text { overflow to process } \\
\text { sewer. }\end{array}$ & $5 W 20$ & $\begin{array}{l}\text { ES93,748.00 } \\
\text { N115,937.77 }\end{array}$ & $\begin{array}{l}\text { Southem portion of } 3706 \text { main } \\
\text { courtyard along the west wall }\end{array}$ & No & Yes \\
\hline 300 & $\mathrm{D}$ & 360 & & 3706 Building - Stuim condensate. & $<0.01$ & $\begin{array}{l}\text { Injection Well with } \\
\text { overflow to process. } \\
\text { sewer. }\end{array}$ & $5 W 20$ & $\begin{array}{l}\text { ES93,780.70 } \\
\text { N115,960.28 }\end{array}$ & $\begin{array}{l}\text { North wall of courtyard that is } \\
\text { accessed via the I'irst Aid Station }\end{array}$ & No & Yes \\
\hline
\end{tabular}

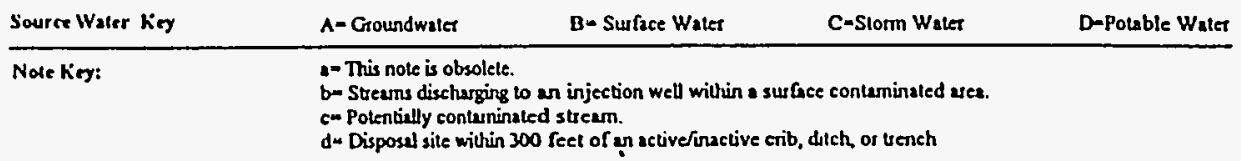


Table 3-1. Miscellaneous Streams Inventory

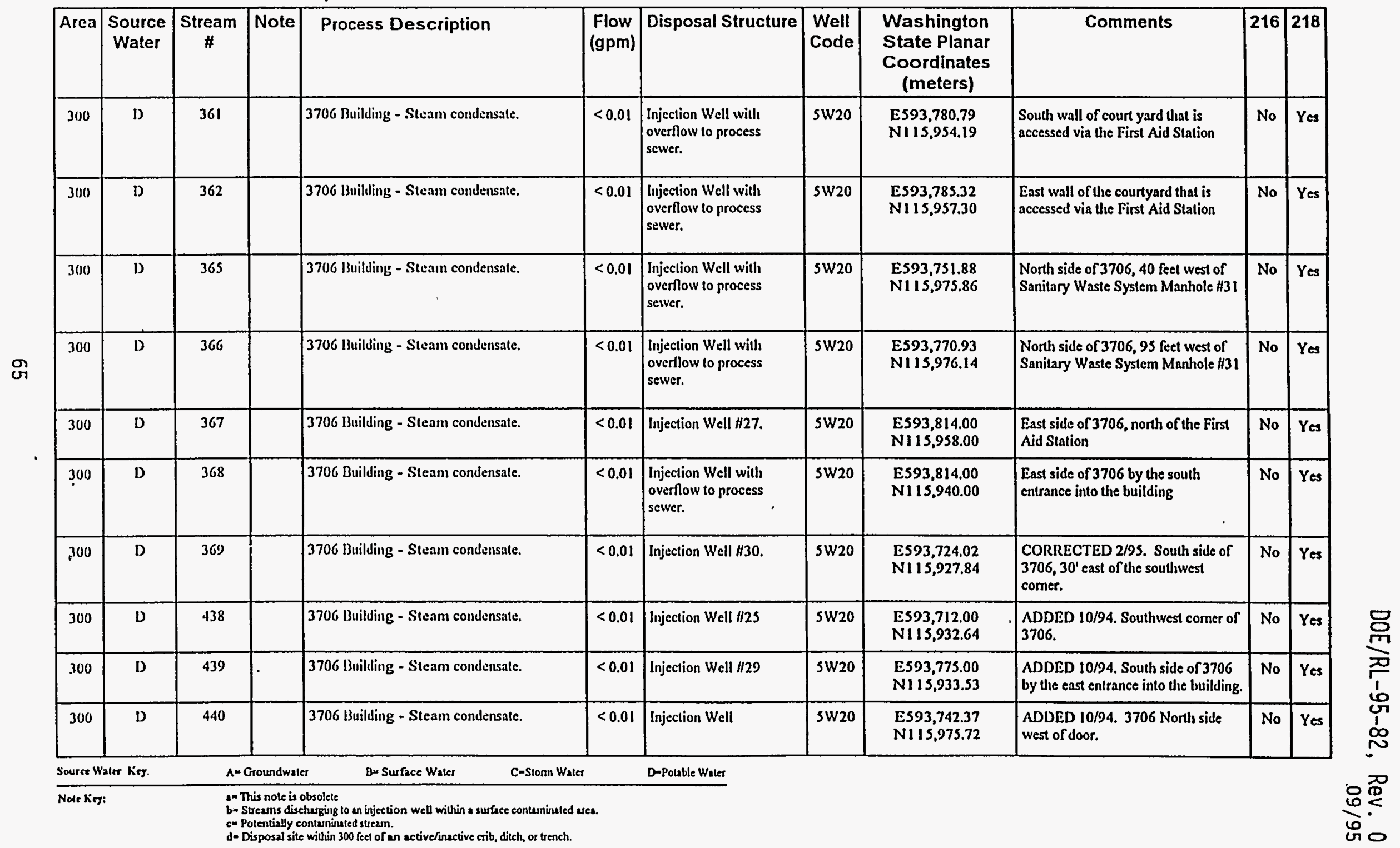


Table 3-1. Miscellaneous Streams Inventory

\begin{tabular}{|c|c|c|c|c|c|c|c|c|c|c|c|}
\hline Area & $\begin{array}{l}\text { Source } \\
\text { Water }\end{array}$ & $\begin{array}{c}\text { Stream } \\
\#\end{array}$ & Note & Process Description & $\begin{array}{c}\text { Flow } \\
\text { (gpm) }\end{array}$ & Disposal Structure & $\begin{array}{l}\text { Well } \\
\text { Code }\end{array}$ & $\begin{array}{c}\text { Washington } \\
\text { State Planar } \\
\text { Coordinates } \\
\text { (meters) }\end{array}$ & Comments & 216 & 218 \\
\hline 300 & $\mathrm{C}$ & 359 & & 3706 Building - Stonn water run-off. & $<0.05$ & Injection Well $1 / 22$ & SD2 & $\begin{array}{l}\text { E: } 593,713.79 \\
\text { N115,975.30 }\end{array}$ & Norlliwest comer of 3706 & Yes & Yes \\
\hline 300 & c & 363 & & 3706 IBuilding - Stonn water nun-ofr. & $<0.0 i$ & $\begin{array}{l}\text { Injection Well with } \\
\text { overflow to process } \\
\text { sewer. }\end{array}$ & SD2 & $\begin{array}{l}E 593,777.70 \\
\text { NI15,957.19 }\end{array}$ & $\begin{array}{l}\text { Central portion of courtyard that is } \\
\text { accessed via the First Aid Station }\end{array}$ & Yes & Yes \\
\hline 300 & C & 364 & & 3706 Building - Stonn waler run-olf. & $<0.01$ & $\begin{array}{l}\text { Injection Well with } \\
\text { overflow to process } \\
\text { sewer. }\end{array}$ & SD2 & $\begin{array}{l}E 593,774.65 \\
\text { N115,957.14 }\end{array}$ & $\begin{array}{l}\text { Central portion of courtyard that is } \\
\text { accessed via the First Aid Station }\end{array}$ & Yes & Yes \\
\hline $3(10)$ & b) & 4.32 & & $\begin{array}{l}3706 \text { A Building - Steam condensate, IIPD. } \\
\text { TRR'-025 }\end{array}$ & $<0.01$ & Injection Well $\$ 28$ & $5 W 20$ & $\begin{array}{l}\text { ES93,819.25 } \\
\text { N115,929.23 }\end{array}$ & $\begin{array}{l}\text { ADDED 10/94. Southeast comer of } \\
3706 \mathrm{~A} \text {. }\end{array}$ & No & Yes \\
\hline 300 & $\mathrm{D}$ & 430 & & $\begin{array}{l}370713 \text { Building - Stenm condensate, IIP'). } \\
\text { TRP-021. }\end{array}$ & $<0.01$ & Injection Well & $5 W 20$ & $\begin{array}{l}\text { ES93,739.00 } \\
\text { NIIS,993.00 }\end{array}$ & $\begin{array}{l}\text { ADDED 2/95. Northeast comer of } \\
\text { 3707B under main steam line labeled } \\
\text { US7. }\end{array}$ & No & Yes \\
\hline 300 & $\mathrm{D}$ & 325 & & 370713 Building - Steam condensate. & $<0.01$ & Injection Well & $5 w 20$ & $\begin{array}{r}E S 93,854.00 \\
N 116,003.00 \\
\end{array}$ & 3707B Building, Southeast & No & Yes \\
\hline 300 & $\mathrm{D}$ & 327 & & 3707B Building - Stearn condensate. & $<0.01$ & $\begin{array}{l}\text { Injection Well, Labelled } \\
\text { as F.D. } \$ 14\end{array}$ & $5 W 20$ & $\begin{array}{l}\text { ES93,846.00 } \\
\text { N } 116,008.00\end{array}$ & 3707B Building, North center & No & Yes \\
\hline 300 & D & 328 & & 370713 Building - Steam condersate. & $<0.01$ & Injection Well & $5 W 20$ & $\begin{array}{l}\text { E:S93,840.00 } \\
\text { N116,009.00 }\end{array}$ & 3707B Building, Northwest Corner & No & Yes \\
\hline 300 & $D$ & 326 & & $\begin{array}{l}\text { 3707B Building - Stean condensate; } \\
\text { currently inactive. }\end{array}$ & $<0.01$ & Injection Well & $s w 20$ & $\begin{array}{l}E S 93,852.00 \\
\text { N } 116,008.00\end{array}$ & $\begin{array}{l}\text { CORRECTED 2/95. Process } \\
\text { Description. 3707B Building. } \\
\text { Northeast comer }\end{array}$ & No & Yes \\
\hline 300 & D & 179 & & $3707 C$ Building - Stean condensale. & 0.10 & Injection Well H24 & $5 W 20$ & $\begin{array}{l}E S 93,701.00 \\
\text { N115,918.74 }\end{array}$ & $\begin{array}{l}\text { CORRECTED 2/95. Process } \\
\text { Description and Comments. 3707C } \\
\text { Center of South Side }\end{array}$ & No & Yes \\
\hline 300 & D & 178 & & 3707C Building - Steanı conderısate. & 0.10 & Injection Well H23 & sW20 & $\begin{array}{l}E S 93,707.33 \\
\text { N115,975.21 }\end{array}$ & $\begin{array}{l}\text { CORRECTED 2/95. Process } \\
\text { Description and Coordinates. } \\
\text { Norticast comer. }\end{array}$ & No & Yes \\
\hline \multicolumn{12}{|c|}{ Sourre Waler kirg } \\
\hline$\kappa$ & & & 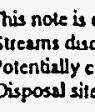 & 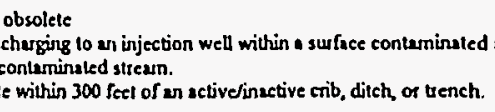 & & & & & & & \\
\hline
\end{tabular}


Table 3-1. Miscellaneous Streams Inventory

\begin{tabular}{|c|c|c|c|c|c|c|c|c|c|c|c|}
\hline Area & $\begin{array}{l}\text { Source } \\
\text { Water }\end{array}$ & $\begin{array}{c}\text { Stream } \\
\#\end{array}$ & Note & Process Description & $\begin{array}{c}\text { Flow } \\
\text { (gpm) }\end{array}$ & Disposal Structure & $\begin{array}{l}\text { Well } \\
\text { Code }\end{array}$ & $\begin{array}{c}\text { Washington } \\
\text { State Planar } \\
\text { Coordinates } \\
\text { (meters) }\end{array}$ & Comments & 216 & 218 \\
\hline 300 & D & 335 & & $3707 \mathrm{C}$ Building - Steam condensate. & $<0.01$ & $\begin{array}{l}\text { Injection Well, Labelled } \\
\text { as F.D. H4 }\end{array}$ & $5 W 20$ & $\begin{array}{l}\text { E } 593,692.00 \\
\text { N115,961.00 }\end{array}$ & 3707C Building West side & No & Yes \\
\hline 300 & $\mathrm{D}$ & 336 & " & 3707C Building - Steam condensate. & $<0.01$ & $\begin{array}{l}\text { Injection Well, Labelled } \\
\text { as F.D. \#3I }\end{array}$ & $5 W 20$ & $\begin{array}{l}\text { ES } 593,704.00 \\
\text { N115,918.72 }\end{array}$ & 3707C Building, Souihwest & No & Yes \\
\hline 300 & D & 337 & & 3707C Building - Steam condensaite. & $<0.01$ & Injection Well & $5 W 20$ & $\begin{array}{l}E 593,701.23 \\
\text { N } 115,975.12 \\
\end{array}$ & 3707C Building, Northeast & No & Yes \\
\hline 300 & $\mathrm{~b}$ & 443 & & 37071) Building - Steam condensale. & $<0.01$ & Injection Well H10 & $5 W 20$ & $\begin{array}{l}\text { E593,874.72 } \\
\text { N } 116,044.56\end{array}$ & $\begin{array}{l}\text { ADDED 10/94. Southwest conier of } \\
\text { 3707D. }\end{array}$ & No & Yes \\
\hline 300 & c & 441 & & 37071) Building - Storn drain. & $<0.01$ & Injection Well & SD2 2 & $\begin{array}{l}\text { ES93,874.30 } \\
\text { N } 116,073.06\end{array}$ & $\begin{array}{l}\text { ADDED 10/94. North side of 3707D } \\
\text { in parking area, labeled DI. }\end{array}$ & Yes & Yes \\
\hline 300 & c & 442 & & 3707D Building - Stom drain. & $<0.01$ & Injection Well & SD2 & $\begin{array}{l}\text { ES93,893.35 } \\
\text { N116,073.33 }\end{array}$ & $\begin{array}{l}\text { ADDED 10/94. Nortlı side of 3707D } \\
\text { in parking area, labeled D2. }\end{array}$ & Yes & Yes \\
\hline 300 & D & 423 & & 3708 Building - Stean condensale. & $<0.01$ & Injection Well & sw20 & $\begin{array}{l}\text { Es93,851.31 } \\
\text { N116,029.89 }\end{array}$ & ADDED 10/94. East side of 3708. & No & Yes \\
\hline 300 & D & 338 & & 3709 Building - Steam condensate. & $<0.01$ & $\begin{array}{l}\text { Injection Well, Labelled } \\
\text { as F.D. } 13\end{array}$ & $5 W 20$ & $\begin{array}{l}\text { Es93,685.24 } \\
\text { N115,974.89 }\end{array}$ & 3709 Building, Northeast & No & Yes \\
\hline 300 & D & 347 & & $\begin{array}{l}3709 \text { A Building - Air compressor } \\
\text { blowdown. }\end{array}$ & $<0.01$ & Injection Well & $5 w 20$ & $\begin{array}{l}\text { ES93,674.00 } \\
\text { N } 115,744.00\end{array}$ & 3709A Building. West side & Yes & Yes \\
\hline 300 & D & 355 & & 3709A Building - Steain trap. & $<0.01$ & Injection Well & $5 W 20$ & $\begin{array}{l}\text { E593,683.76 } \\
\text { N } 115,737.12 \\
\end{array}$ & 3709 A Building, South side & No & Yes \\
\hline .300 & D & 343 & & 3711 Building - Steam condensate. & $<0.01$ & Injection Well & $5 W 20$ & $\begin{array}{l}\text { ES94,027.10 } \\
\text { N116,046.18 } \\
\end{array}$ & 3711 Building, North side. & No & Yes \\
\hline 300 & D & 433 & & 3711 Building - Steam condensate. & $<0.01$ & Injection Well & $5 w_{20}$ & $\begin{array}{l}\text { ES94,032.01 } \\
\text { N116,022.63 } \\
\end{array}$ & ADDED 10/94. South side of 3711. & No & Yes \\
\hline 300 & D & 351 & & 3712 Building - Steam condensatc. & $<0.01$ & Injection Well & $5 w 20$ & $\begin{array}{l}E 593,925.75 \\
\text { N } 116,149.86\end{array}$ & 3712 Building, East center & No & Yes \\
\hline
\end{tabular}

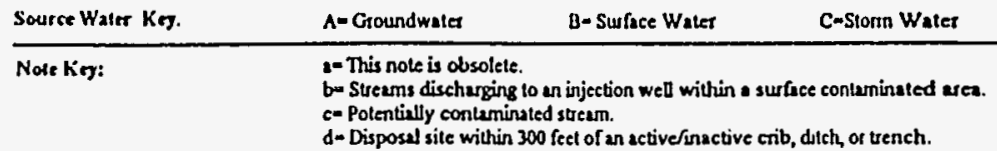

D-Polable Water

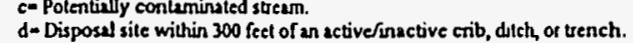


Table 3-1. Miscellaneous Streams Inventory

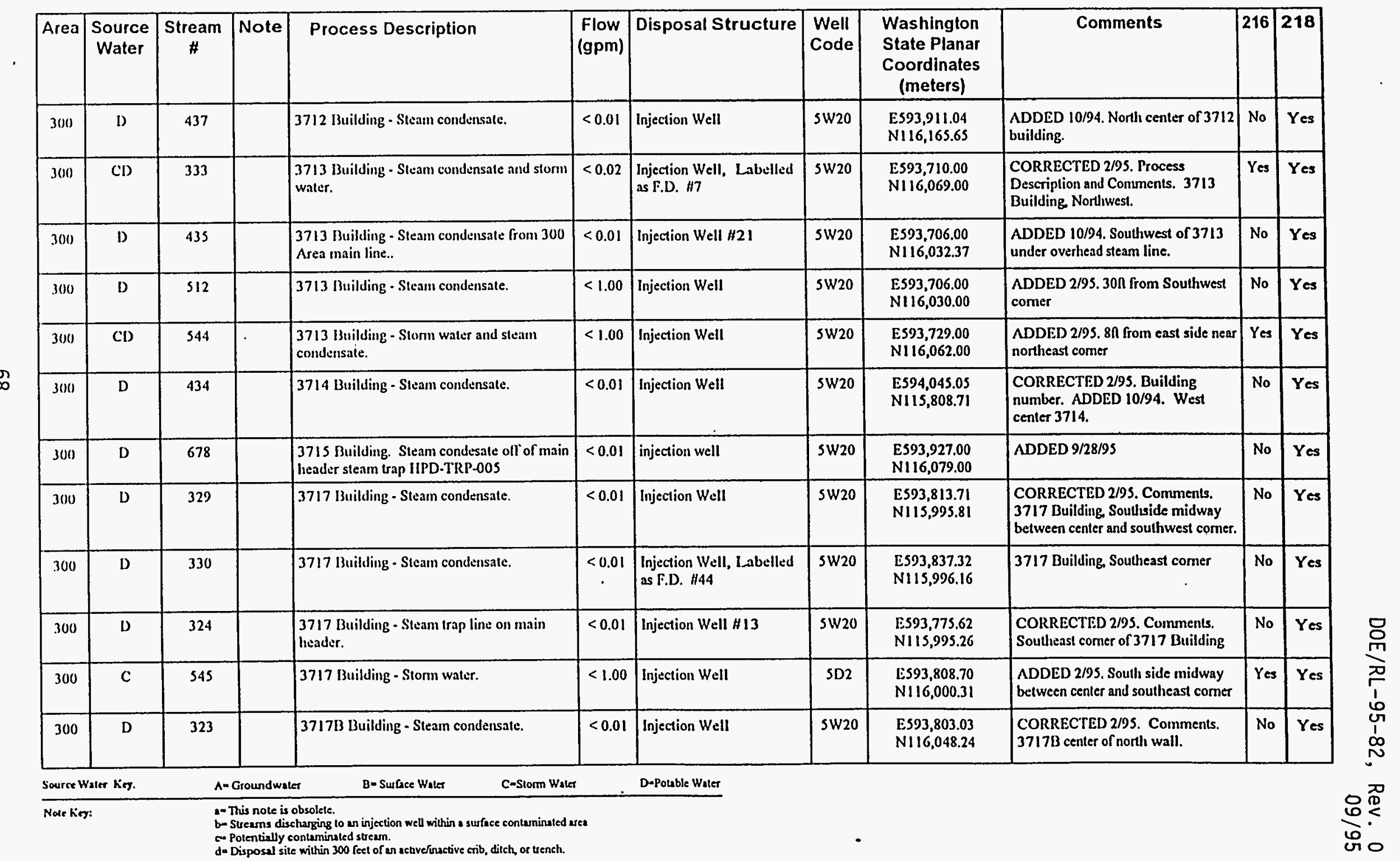


Table 3-1. Miscellaneous Streams Inventory

\begin{tabular}{|c|c|c|c|c|c|c|c|c|c|c|c|}
\hline Area & $\begin{array}{l}\text { Source } \\
\text { Water }\end{array}$ & $\begin{array}{c}\text { Stream } \\
\#\end{array}$ & Note & Process Description & $\begin{array}{c}\text { Flow } \\
\text { (gpm) }\end{array}$ & Disposal Structure & $\begin{array}{l}\text { Well } \\
\text { Code }\end{array}$ & $\begin{array}{l}\text { Washington } \\
\text { State Planar } \\
\text { Coordinates } \\
\text { (meters) }\end{array}$ & Comments & 216 & 218 \\
\hline 300 & $\mathrm{D}$ & 180 & & 3717B Builuing - steram condensitc. & 0.00 & Injection Well & sw20 & $\begin{array}{l}\text { E593,798.94 } \\
\text { N116,048.91 }\end{array}$ & $\begin{array}{l}\text { ELIMINATED 10/94. South side of } \\
\text { 3717B. }\end{array}$ & No & No \\
\hline 3160 & $D$ & 340 & & 3718 Building - Steam condensale. & $<0.01$ & $\begin{array}{l}\text { Injection Well, Labelled } \\
\text { as F.D. H40 }\end{array}$ & $5 W 20$ & $\begin{array}{l}\text { E594,095.21 } \\
\text { N115,947.36 }\end{array}$ & 3718 Building, Southwest comer & No & Yes \\
\hline 300 & c & 270 & & $\begin{array}{l}\text { 3718 } \mathrm{A} \text { Building roof stonm water runofr. } \\
\text { Drains are piped into ground and directed } \\
\text { away from } 340 \text { Building. }\end{array}$ & $<0.50$ & Injection Well & SD2 & $\begin{array}{l}\text { E594,101.13 } \\
\text { N115,876.19 }\end{array}$ & ELIMINATED 10/94. & No & No \\
\hline 300 & D & 436 & & $\begin{array}{l}3722 \text { Building - Sleam condensale, IIIPD- } \\
\text { T'RP-013, } 014 \text {. }\end{array}$ & $<0.01$ & Injection Well $H 6$ & sw20 & $\begin{array}{l}\text { E593,745.55 } \\
\text { N116,071.02 }\end{array}$ & $\begin{array}{l}\text { ADDED 10/94. Norliswest comer of } \\
3722 \text {. }\end{array}$ & No & Yes \\
\hline 300 & D & 383 & & 3730 [Building - Steam condensite. & $<0.01$ & lnjection Well & SW20 & $\begin{array}{l}\text { E593,821.00 } \\
\text { N } 115,908.00\end{array}$ & $\begin{array}{l}3730 \text { building, near the southwest } \\
\text { comer }\end{array}$ & No & Yes \\
\hline 300 & D & 420 & & 3730 Building - Steam condensale. & $<0.01$ & Injeclion Well & sw20 & $\begin{array}{l}\text { ES93,842.45 } \\
\text { NIIS,958.13 }\end{array}$ & $\begin{array}{l}\text { ELIMINATED. Stream routed to the } \\
\text { process sewer, per Lazarski } 9 / 28 / 95 \text {. } \\
\text { ADDED 10/94. Northwest comer of } \\
3730 \text {. }\end{array}$ & No & No \\
\hline 300 & $\mathrm{D}$ & 421 & & 3730 Building - Steam condensitle. & $<0.01$ & Injection Well & $5 W 20$ & $\begin{array}{l}\text { ES93,856.67 } \\
\text { NI15,948.83 }\end{array}$ & $\begin{array}{l}\text { ADDED 10/94. Northeast corner of } \\
3730 \text {. }\end{array}$ & No & Yes \\
\hline 300 & C & 517 & & 3731 Building - Stom waler run-off. & $<1.00$ & Injection Well & SD2 & $\begin{array}{l}\text { ES94,132.21 } \\
\text { N116,030.00 }\end{array}$ & $\begin{array}{l}\text { ADDED 2/95. } 3731 \text { Building } \\
\text { northeast comer roof drain. }\end{array}$ & Yes & Yes \\
\hline 300 & C & 518 & & 373 I Building - Storm waler nun-oll: & $<1.00$ & Injection Well & SD2 & $\begin{array}{l}\text { E594, } 132.39 \\
\text { N116,010.03 }\end{array}$ & $\begin{array}{l}\text { ADDED 2/95. } 373 \text { I Building } \\
\text { southeast comer roof drain. }\end{array}$ & Yes & Yes \\
\hline 300 & D) & 269 & & 37.31 Building - sleam condensalc. & $<0.50$ & Injection Well & SD2 & $\begin{array}{l}\text { E:594,132.25 } \\
\text { N116,019.53 }\end{array}$ & $\begin{array}{l}\text { DELETED stomwater as a source } \\
\text { 9/28/95. CORRECTED } 2 / 95 \text {. } \\
\text { Process Description and Commenls. } \\
3731 \text { Building center of east side }\end{array}$ & No & Yes \\
\hline 300 & $\mathrm{D}$ & 349 & & $\begin{array}{l}3732 \text { Building - Steam condensate from } \\
\text { quench tank. }\end{array}$ & $<0.01$ & Injection Well & $5 W 20$ & $\begin{array}{l}E 593,830.00 \\
\text { N116,054.00 }\end{array}$ & 3732 Building, SW corner & No & Yes \\
\hline
\end{tabular}

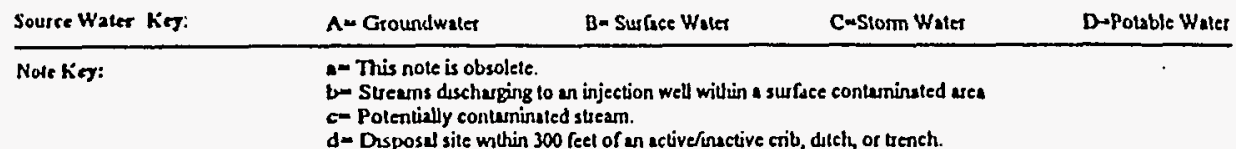


Table 3-1. Miscellaneous Streams Inventory

\begin{tabular}{|c|c|c|c|c|c|c|c|c|c|c|c|}
\hline Area & $\begin{array}{l}\text { Source } \\
\text { Water }\end{array}$ & $\begin{array}{c}\text { Stream } \\
\#\end{array}$ & Note & Process Description & $\begin{array}{l}\text { Flow } \\
(\mathrm{gpm})\end{array}$ & Disposal Structure & \begin{tabular}{c|} 
Well \\
Code
\end{tabular} & $\begin{array}{l}\text { Washington } \\
\text { State Planar } \\
\text { Coordinates } \\
\text { (meters) }\end{array}$ & Comments & 216 & 218 \\
\hline 300 & D) & 419 & & 3732 Iuilding - Stcan condensate. & $<0.01$ & Injection Well HIS & sW20 & $\begin{array}{l}E 593,826.00 \\
N 116,072.27\end{array}$ & $\begin{array}{l}\text { ADDED 10/94. Northwest comer of } \\
3732 \text {. }\end{array}$ & No & Yes \\
\hline 300 & D & 334 & & $\begin{array}{l}3734 \text { Building - Stean condensite from } \\
\text { main header. }\end{array}$ & $<0.01$ & $\begin{array}{l}\text { lujection Well, Labelled } \\
\text { as F.D. H8 }\end{array}$ & $5 W 20$ & $\begin{array}{l}\text { ES93,680.00 } \\
\text { N116,051.00 }\end{array}$ & 3734 Building. & No & Yes \\
\hline 300 & D & s!9 & & 3734A Building - Steam condensale. & $<1.00$ & Injection Well & $5 w 20$ & $\begin{array}{l}\text { ES93,679.76 } \\
\text { N } 116,040.00\end{array}$ & $\begin{array}{l}\text { ADDED 2/95. 3734A Building } \\
\text { south side. }\end{array}$ & No & Yes \\
\hline 300 & D & 397 & & 3745 Building - Steam condensate. & $<0.05$ & Injection Well HI & $5 W 20$ & $\begin{array}{l}\text { E } 593,729.01 \\
\text { NIIS,899.33 }\end{array}$ & $\begin{array}{l}\text { ADDED 10/94. Northeast corner of } \\
3745\end{array}$ & No & Yes \\
\hline 300 & $\mathrm{D}$ & 308 & & 37.45 Building - Stean condensate. & $<0.05$ & Injection Well HS & $5 W 20$ & $\begin{array}{l}\text { ES93,729.43 } \\
\text { N } 115,870.68\end{array}$ & ADDED 10/94. East side of 3745 & No & Yes \\
\hline 300 & D & 399 & & 3745 Building - Steam condensiate. & $<0.05$ & Injection Well H2 & $5 W 20$ & $\begin{array}{l}\text { ES93,725.07 } \\
\text { N115,856.60 }\end{array}$ & $\begin{array}{l}\text { ADDED 10/94. } 30 \text { feet south of } \\
3745\end{array}$ & No & Yes \\
\hline 300 & $\mathrm{D}$ & 380 & & 3745A Building - Steam condensate. & $<0.01$ & Injection Well & $5 w 20$ & $\begin{array}{l}\text { ES93,667.28 } \\
\text { N115,899.95 }\end{array}$ & $\begin{array}{l}\text { ELIMINATED 3/95. This stream } \\
\text { routed to the process sewer. 3745A } \\
\text { Building, West side }\end{array}$ & No & No \\
\hline 300 & $\mathrm{D}$ & 379 & & $\begin{array}{l}\text { 374513 Building - French drain; steam } \\
\text { condensate. }\end{array}$ & $<0.01$ & Injection Well & $5 W 20$ & $\begin{array}{l}\text { ES93,666.75 } \\
\text { N115,936.52 }\end{array}$ & $\begin{array}{l}\text { ELIMINATED } 3 / 95 \text {. This stream } \\
\text { rouled to the process sewer. } \\
\text { Northeast comer of } 3745 B \text { Building }\end{array}$ & No & No \\
\hline 300 & $\mathrm{D}$ & 491 & & 3762 Building - Steam condensate. & $<0.01$ & Injection Well $H 42$ & $5 W 20$ & $\begin{array}{l}\text { ES94,095.41 } \\
\text { N } 115,233.28\end{array}$ & $\begin{array}{l}\text { ADDED 10/94. Northeast of } \\
\text { building } 3762\end{array}$ & No & Yes \\
\hline 300 & $\mathrm{D}$ & 345 & & 3765 I Building - IIVAC condensate. & $<0.01$ & Injection Well & $5 w_{20}$ & $\begin{array}{l}\text { F:594,280.97 } \\
\text { N115,621.36 }\end{array}$ & 3765 Building, Southwest comer & No & Yes \\
\hline 300 & D & 446 & & 377 Building - Steam condensate. & $<0.05$ & Injection Well $\# 36$ & $5 W 20$ & $\begin{array}{l}\text { Es93,653.66 } \\
\text { N } 116,174.53\end{array}$ & ADDED 10/94. North of 377. & No & Yes \\
\hline 300 & c & 373 & & 3790 Building - Receives stom water. & $<0.01$ & Injection Well & $5 w 20$ & $\begin{array}{l}1: 594,019.24 \\
\text { NIIS,594.20 }\end{array}$ & Southwest 3790 & No & Yes \\
\hline
\end{tabular}

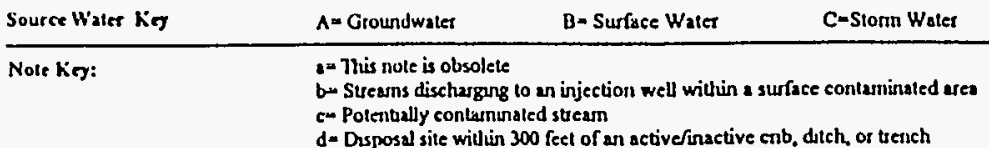

D-Potable Walet 
Table 3-1. Miscellaneous Streams Inventory

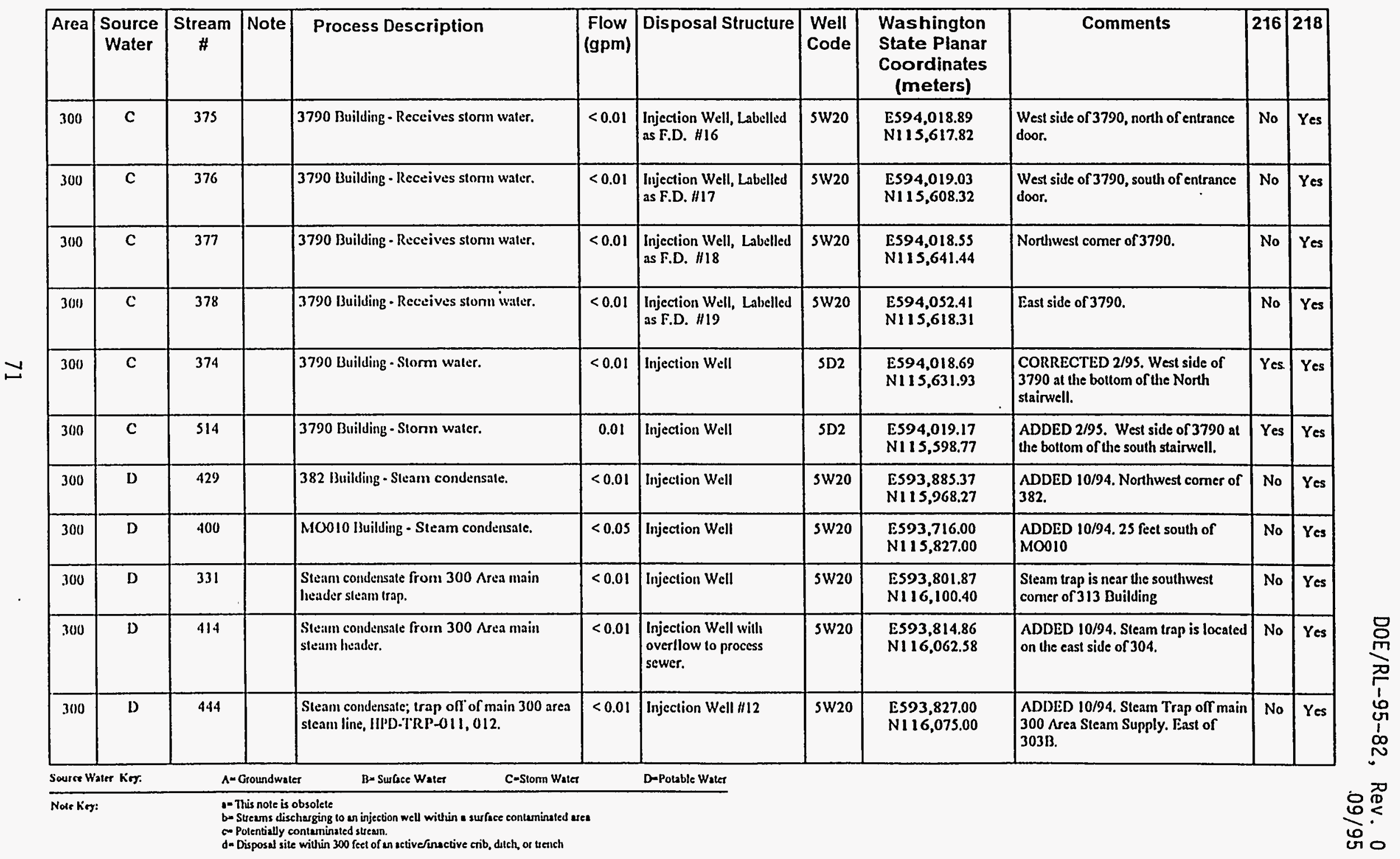


Table 3-1. Miscellaneous Streams Inventory

\begin{tabular}{|c|c|c|c|c|c|c|c|c|c|c|c|}
\hline Area & $\begin{array}{l}\text { Source } \\
\text { Water }\end{array}$ & $\begin{array}{c}\text { Stream } \\
\#\end{array}$ & Note & Process Description & $\begin{array}{c}\text { Flow } \\
\text { (gpm) }\end{array}$ & Disposal Structure & $\begin{array}{l}\text { Well } \\
\text { Code }\end{array}$ & $\begin{array}{l}\text { Washington } \\
\text { State Planar } \\
\text { Coordinates } \\
\text { (meters) }\end{array}$ & Comments & 216 & 218 \\
\hline 300 & i) & 339 & & $\begin{array}{l}\text { Steam condensate; trap olf of main } 300 \text { area } \\
\text { stean line. }\end{array}$ & $<0.01$ & $\begin{array}{l}\text { lujection Well, Labelled } \\
\text { as F.D. } 1 / 26\end{array}$ & $5 W 20$ & $\begin{array}{l}\text { E: } 593,802.66 \\
\text { N116,052.80 }\end{array}$ & $\begin{array}{l}\text { Located off southeast corner of } 303 \mathrm{~A} \\
\text { Building }\end{array}$ & No & Yes \\
\hline 300 & D & 332 & & $\begin{array}{l}\text { West high tank overflow and sleam } \\
\text { condensate trap. }\end{array}$ & $<0.01$ & lnjection Well & $5 w 20$ & $\begin{array}{l}\text { E594,037.19 } \\
\text { N1 } 16,008.63\end{array}$ & 300 Asca & Yes & Yes \\
\hline 3000 & c & 674 & & $\begin{array}{l}1226 \text { Building. hijection well out of service, } \\
\text { but may collect storm water. }\end{array}$ & $<0.01$ & injection well & SD2 & $\begin{array}{l}\text { Es94, } 117.00 \\
\text { N111,126.00 }\end{array}$ & $\begin{array}{l}\text { Added 8/21/95 per cc:mail message } \\
\text { from } M \text {. Gunter dated } 8 / 18 / 95\end{array}$ & Yes & Yes \\
\hline 3000 & 1) & 49 & & $\begin{array}{l}1240 \text { Building steam condensate and } \\
\text { welding torch cooling water (sanitary, closed } \\
\text { system). }\end{array}$ & $<0.00$ & Injection Well & $5 A 19$ & $\begin{array}{l}\text { ES94,061.63 } \\
\text { NIII,424.42 }\end{array}$ & $\begin{array}{l}\text { CORRECTED: } 67 / 6 / 95 \text {, stream is is } \\
\text { active per cc:mail from } M \text {. Gunter } \\
\text { dated } 6 / 30 / 95 \text {. Indicated that stream } \\
\text { was inactive on } 8 / 94\end{array}$ & No & Yes \\
\hline 400 & c & 37 & & 403 ISSF - E:llluent consists of rninwater. & $<0.01$ & Injection Well & 5D2 & $\begin{array}{l}E 587,648.59 \\
\text { N } 123,216.67 \\
\end{array}$ & & Yes & Yes \\
\hline 400 & $\mathrm{CD}$ & 19 & c & $\begin{array}{l}408 \text { South dump heal exchanger and } 491-W \\
\text { Heal Transport Building, west side - Eflluent } \\
\text { originales from rainwater and rheostal water. } \\
\text { Contains nonregulated quantities of sodium } \\
\text { carbonale. }\end{array}$ & $<0.01$ & Injection Well H0S & SD4, & $\begin{array}{l}\text { E587,539.32 } \\
\text { N123,055.90 }\end{array}$ & & Yes & Yes \\
\hline .100 & c & 17 & & $\begin{array}{l}408 \AA \text { lenst dump heal exchanger - Ellluent } \\
\text { consists of rainwater. }\end{array}$ & $<0.01$ & Injection Wsll 1103 & SD2 & $\begin{array}{l}\text { E } 587,701.33 \\
\text { N123,081.87 } \\
\end{array}$ & & Yes & Yes \\
\hline 400 & c & 20 & & $\begin{array}{l}408 \mathrm{C} \text { West dump heat exclsanger - Ellluent } \\
\text { originales from rainwater. }\end{array}$ & $<0.01$ & Injection Well 1106 & SD2 & $\begin{array}{l}\text { E.587,531.66 } \\
\text { N123,071.00 }\end{array}$ & $\begin{array}{l}\text { Eflluent will be re-routed to stream } \\
H 19 .\end{array}$ & Yes & Yes \\
\hline 400 & c & 26 & & $\begin{array}{l}\text { 45313 Switch Gear Pad - Eflluenl originates } \\
\text { from rainwaler. }\end{array}$ & $<0.01$ & Injection Well IIII & SD2 & $\begin{array}{l}\text { ES87, } 544.84 \\
\text { N123,043.00 }\end{array}$ & & Yes & Yes \\
\hline 400 & c & 27 & & $\begin{array}{l}\text { 453C Switch Gear Pad - Eflluent originales } \\
\text { from rainwaler. }\end{array}$ & $<0.01$ & Injection Well H07 & SD2 & $\begin{array}{l}\text { E587,559.50 } \\
\text { N123,143.91 }\end{array}$ & & Yes & Yes \\
\hline 400 & $\mathrm{CD}$ & 16 & & $\begin{array}{l}\text { 4621E Auxiliary E(puipment Building - } \\
\text { Water condensate from IIVAC system and } \\
\text { rain water. }\end{array}$ & $<0.01$ & Injection Well H02 & SD2, & $\begin{array}{l}\text { E } 587,665.47 \\
\text { N123,160.26 }\end{array}$ & & Yes & Yes \\
\hline
\end{tabular}


Table 3-1. Miscellaneous Streams Inventory

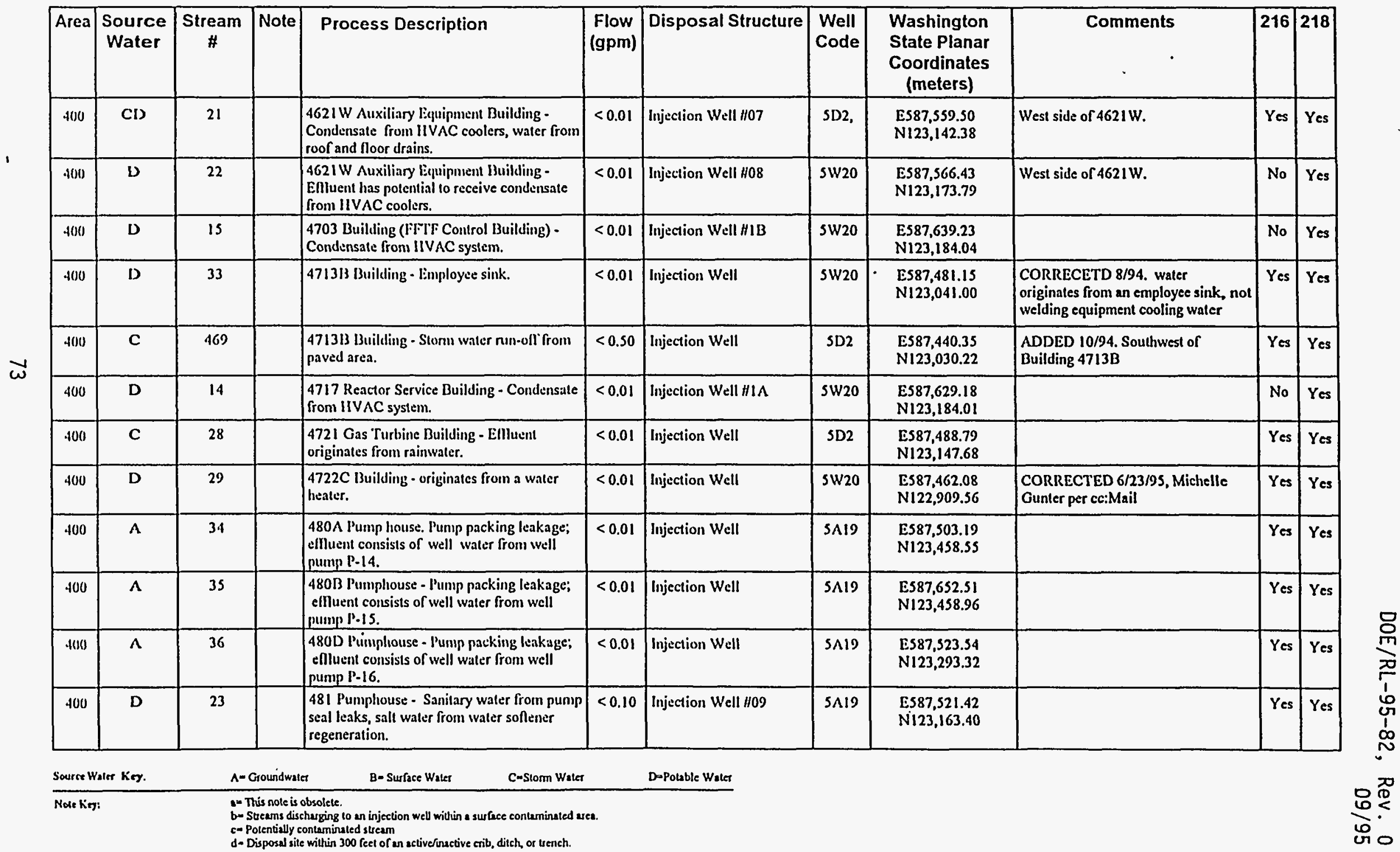


Table 3-1. Miscellaneous Streams Inventory

\begin{tabular}{|c|c|c|c|c|c|c|c|c|c|c|c|}
\hline Area & $\begin{array}{l}\text { Source } \\
\text { Water }\end{array}$ & $\begin{array}{c}\text { Stream } \\
\#\end{array}$ & Note & Process Description & $\begin{array}{c}\text { Flow } \\
\text { (gpm) }\end{array}$ & Disposal Structure & $\begin{array}{l}\text { Well } \\
\text { Code }\end{array}$ & $\begin{array}{l}\text { Washington } \\
\text { State Planar } \\
\text { Coordinates } \\
\text { (meters) }\end{array}$ & Comments & 216 & 218 \\
\hline 400 & $\mathrm{c}$ & 25 & & $\begin{array}{l}\text { 482A Building - T-58 Waler Storage Tank } \\
\text { and Equipment Room - Rainwater off water } \\
\text { storage tank. }\end{array}$ & $<0.01$ & Injection Well $/ 10$ & SD2 & $\begin{array}{l}\text { E587,516.25 } \\
\text { N123,181.09 }\end{array}$ & & Yes & Yes \\
\hline 400 & $c$ & 24 & & $\begin{array}{l}\text { 482A Building - T-87 Waler Storage and } \\
\text { Tank Equipment Room - Rainwaler off } \\
\text { water slorage lank. }\end{array}$ & $<0.01$ & Injection Well $\# 10 \Lambda$ & SD2 & $\begin{array}{l}\text { E587,546.28 } \\
\text { N123,186.17 }\end{array}$ & & Yes & Yes \\
\hline 400 & (D) & 18 & c & $\begin{array}{l}\text { 49IE Heat Transport Building, east side - } \\
\text { Eflluent originates from rainwvaler off roof of } \\
\text { IITS-E and condensate from } \mathrm{HVAC} \text { system. } \\
\text { Consists of rheostat water and nonregulated } \\
\text { quantities of sodium carbonale. }\end{array}$ & $<0.01$ & Injection Well HO4 & SD4, & $\begin{array}{l}\text { E587,655.13 } \\
\text { N123,053.66 }\end{array}$ & . & Yes & Yes \\
\hline 400 & $\mathrm{C}$ & 31 & & $\begin{array}{l}\text { Altitude Valve Pit T-58 - Ellhuent consists of } \\
\text { rainwater. }\end{array}$ & $<0.01$ & Injection Well & 5D2 & $\begin{array}{l}E 587,560.07 \\
\text { N120,438.71 } \\
\end{array}$ & & Yes & Yes \\
\hline 400 & $\mathrm{C}$ & 32 & & $\begin{array}{l}\text { Alitude Valve Pit T-87 - Eflluent consists } \\
\text { of rainwater. }\end{array}$ & $<0.01$ & Injection Well & SD2 & $\begin{array}{l}\text { ES87,529.60 } \\
\text { N120,438.63 } \\
\end{array}$ & & Yes & Yes \\
\hline 400 & $c$ & 30 & & $\begin{array}{l}\text { Altitude Valve T-330 - Eftluent consists of } \\
\text { rainwater. }\end{array}$ & $<0.01$ & Injection Well & 5D2 & $\begin{array}{l}\text { ES89,368.92 } \\
\text { N123,193.88 } \\
\end{array}$ & & Yes & Yes \\
\hline 600 & $D$ & 13 & & $\begin{array}{l}251 W \text { (sulsstation) - Continuous discharge } \\
\text { of sanitary water. Discharge is from the } \\
\text { automaled hypo-chlorination being used. }\end{array}$ & 6.00 & & - & $\begin{array}{l}\text { ES69,913.01 } \\
\text { N138,906.71 }\end{array}$ & $\begin{array}{l}\text { ELIMINATED 9/28/95 discharges } \\
\text { directly to ground. CORRECTED } \\
6 / 28 / 95 \text {, per D. Herman this does not } \\
\text { discharge to an injection well. } \\
\text { corrected } 8 / 94 \text {. Process was updated. }\end{array}$ & No & No \\
\hline 700 & c & 656 & & $\begin{array}{l}712 \text { B Building - This system collects storn } \\
\text { water from the areas surrounding the } 712 \\
\text { buildings. }\end{array}$ & $<0.05$ & Infiltration Trench & $\mathrm{N} / \mathrm{A}$ & $\begin{array}{l}\text { ES91,515.00 } \\
N 124,100.00\end{array}$ & $\begin{array}{l}\text { ADDED 6/95. This trencli is } \\
\text { scheduled to be constructed in the } \\
\text { summer of } 1995 \text {. }\end{array}$ & Yes & No \\
\hline
\end{tabular}

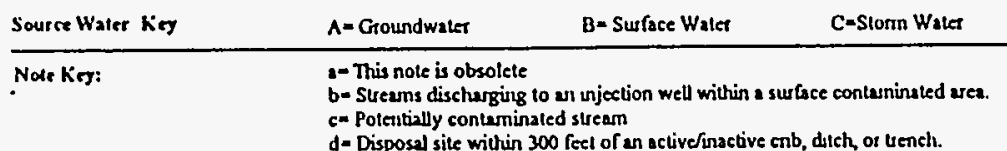

D.Potable Water 
DOE/RL-95-82, Rev. 0

$09 / 95$

\subsection{REFERENCES}

DOE, 1987, "PT an and ScheduTe to Discontinue Disposal of Contaminated Liquids into the Soil Column at the Hanford Site," DOE-065, Response to Congressional Request, U.S. Department of Energy, Richland Operations Office, Richland, Washington.

DOE, 1994, "Plan and ScheduTe for Disposition and Regulatory Compliance for Misce17aneous Streams," DOE/RL-93-94, Rev. 1, U.S. Department of Energy, Richland Operations Office, Richland, Washington.

Ecology, EPA, and DOE, 1994, "Hanford Federal Facility Agreement and Consent Order," Washington State Department of Ecology, U.S. Environmental Protection Agency, and U.S. Department of Energy, O1 ympia, Washington.

Ecology, and U.S. DOE, 1991, "Consent Order No. DE91N-177," Washington Department of Ecology, and U.S. Department of Energy, Olympia, Washington. 
DOE/RL-95-82, Rev. 0

$09 / 95$

(This page intentionally left blank) 
DOE/RL-95-82, Rev. 0 $09 / 95$

\section{APPENDIX A}

Miscellaneous Streams Inventory Area Maps

$A-i$ 
DOE/RL-95-82, Rev. 0

09/95

(This page intentionally left blank) 
Appendix A

The following area maps describes the locations of the active Hanford Site Miscellaneous Streams. The stream id numbers on the area maps corresponds to the "Streams \#" field on the Miscellaneous Streams Inventory. Area maps are included for the 100, 200, 300, and 400 Areas. Area maps were not available for the remaining locations.

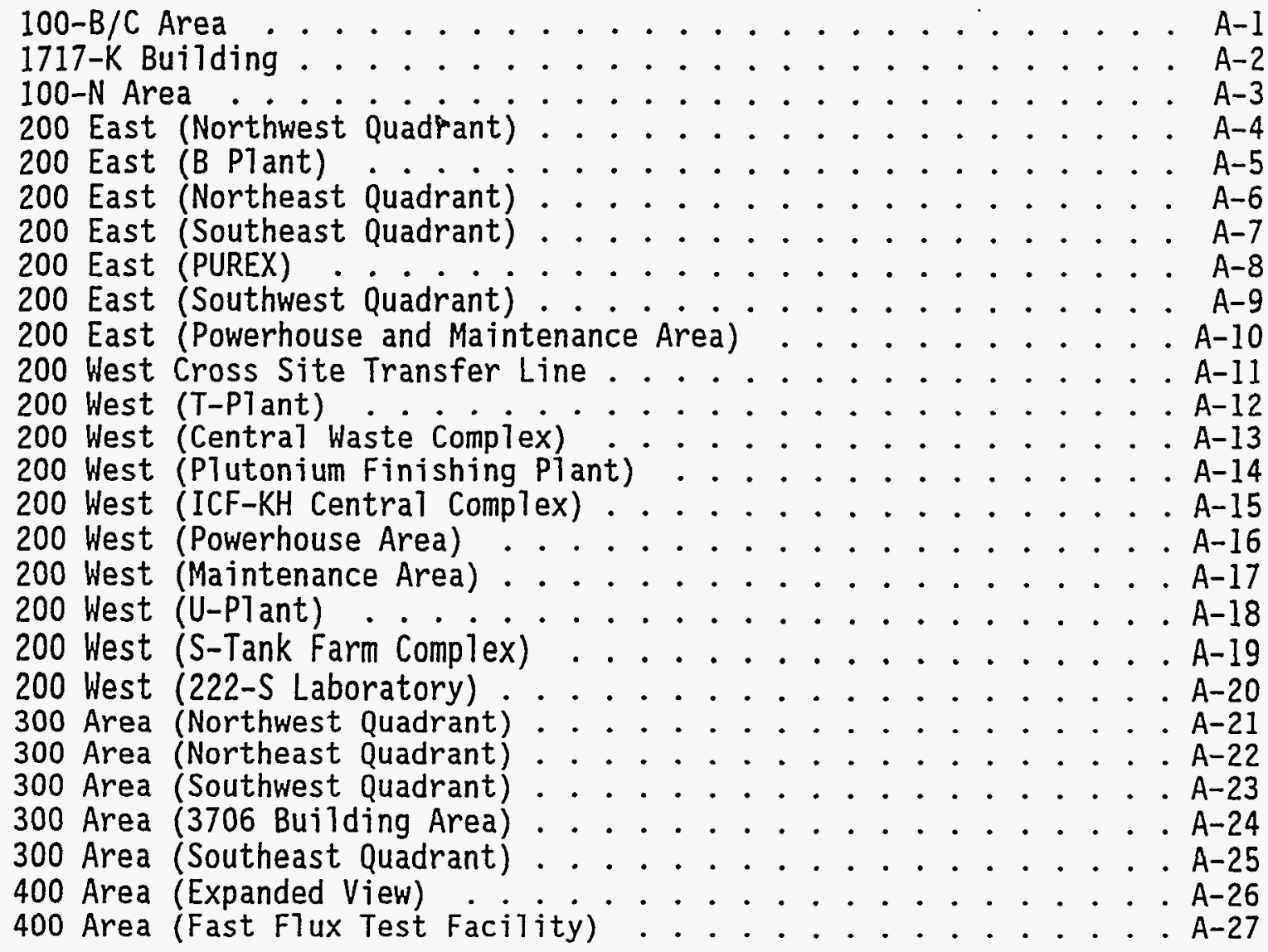


D0E/RL-95-82, Rev. 0

$09 / 95$

(This page intentionally left blank) 


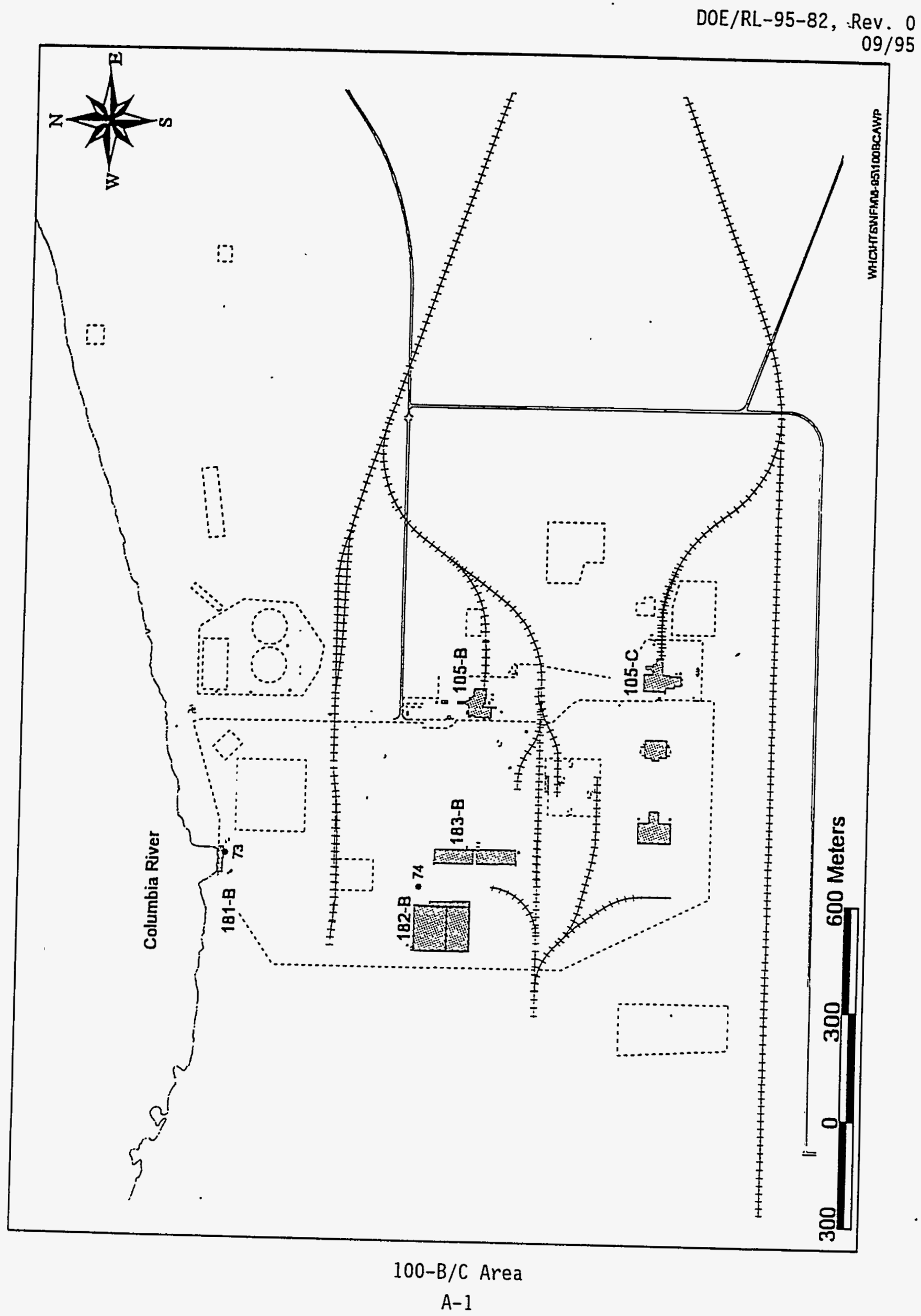




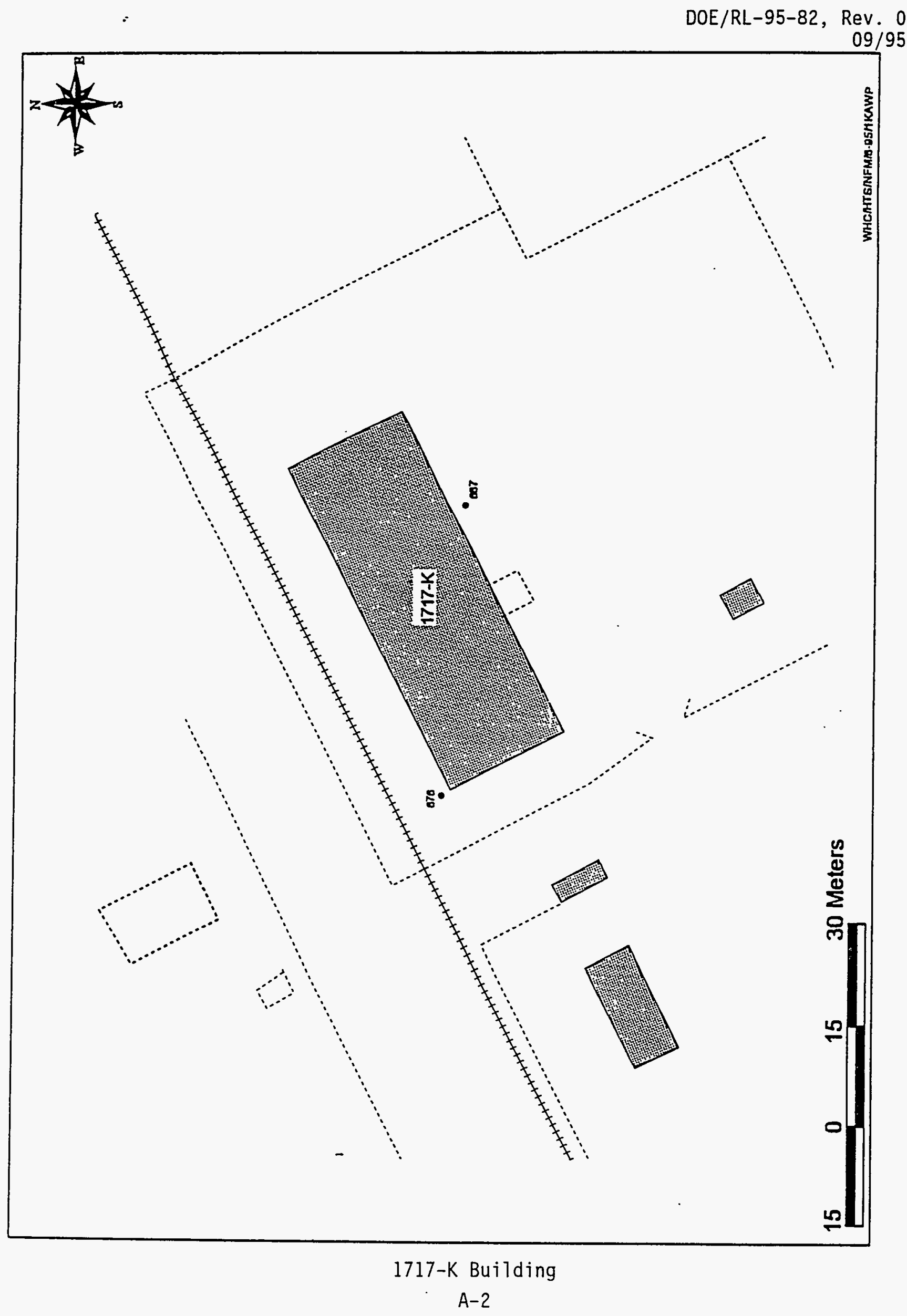


DOE/RL-95-82, Rev. 0

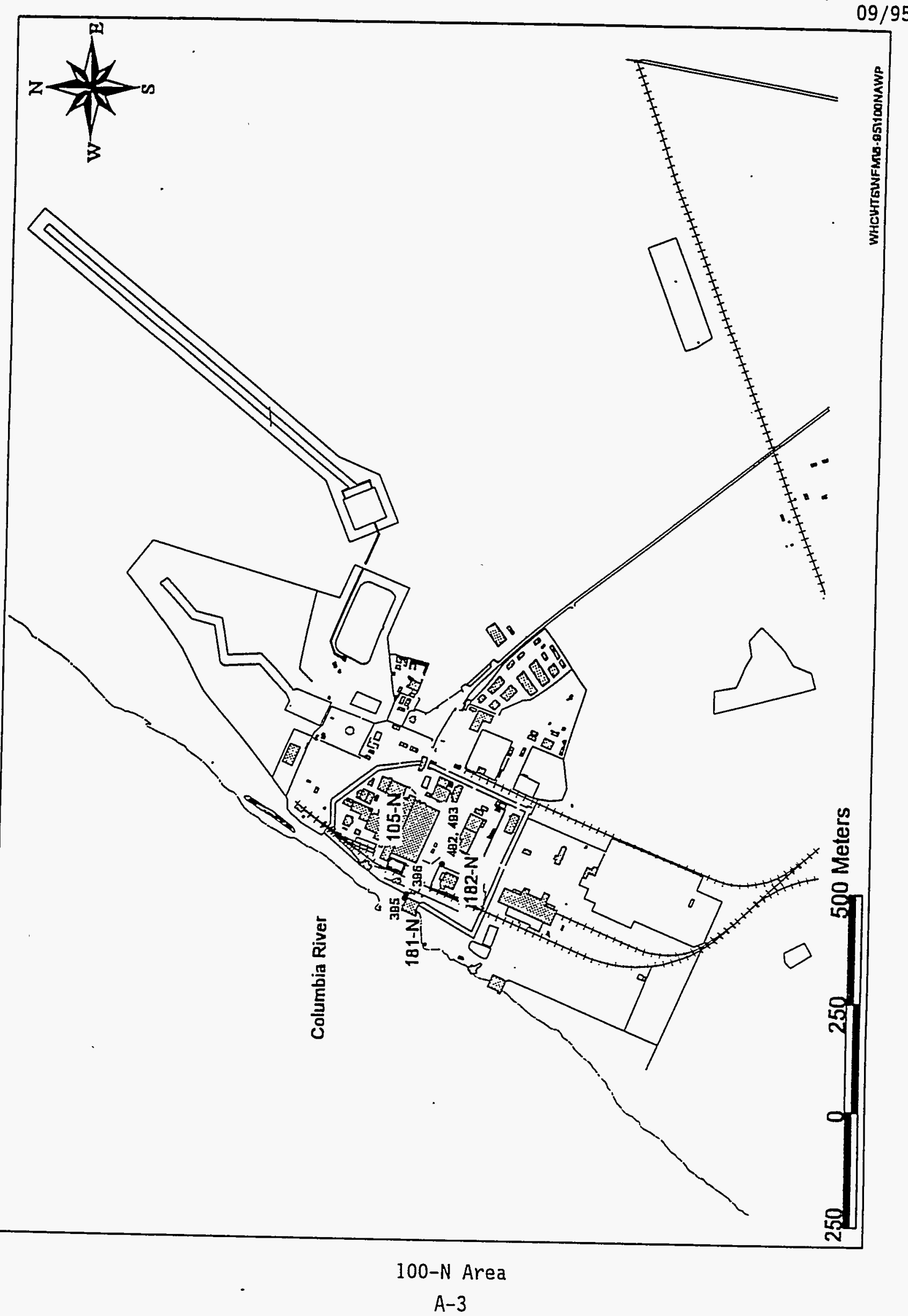


DOE/RL-95-82, Rev. 0

$09 / 95$

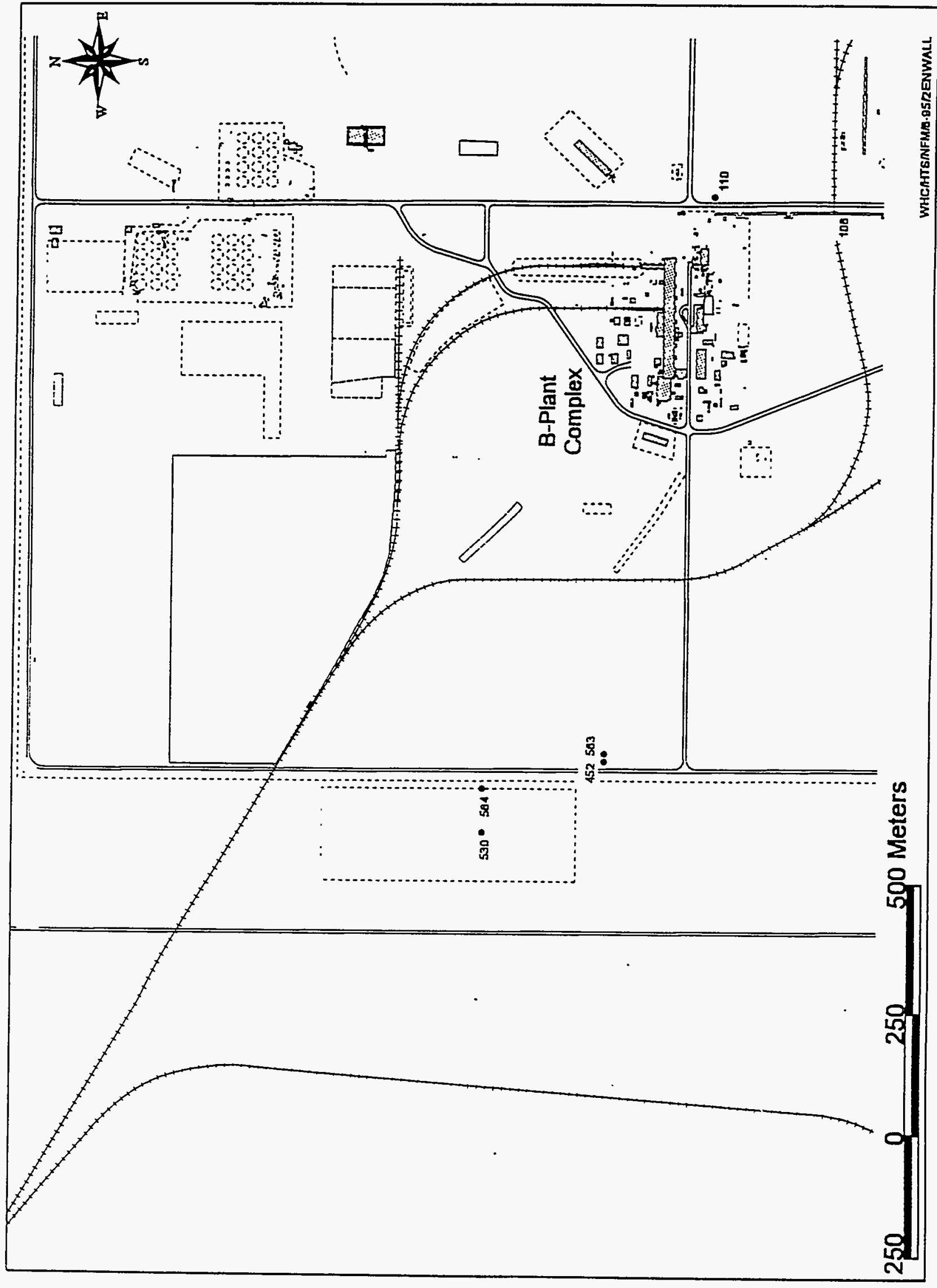

200 East (Northwest Quadrant) 


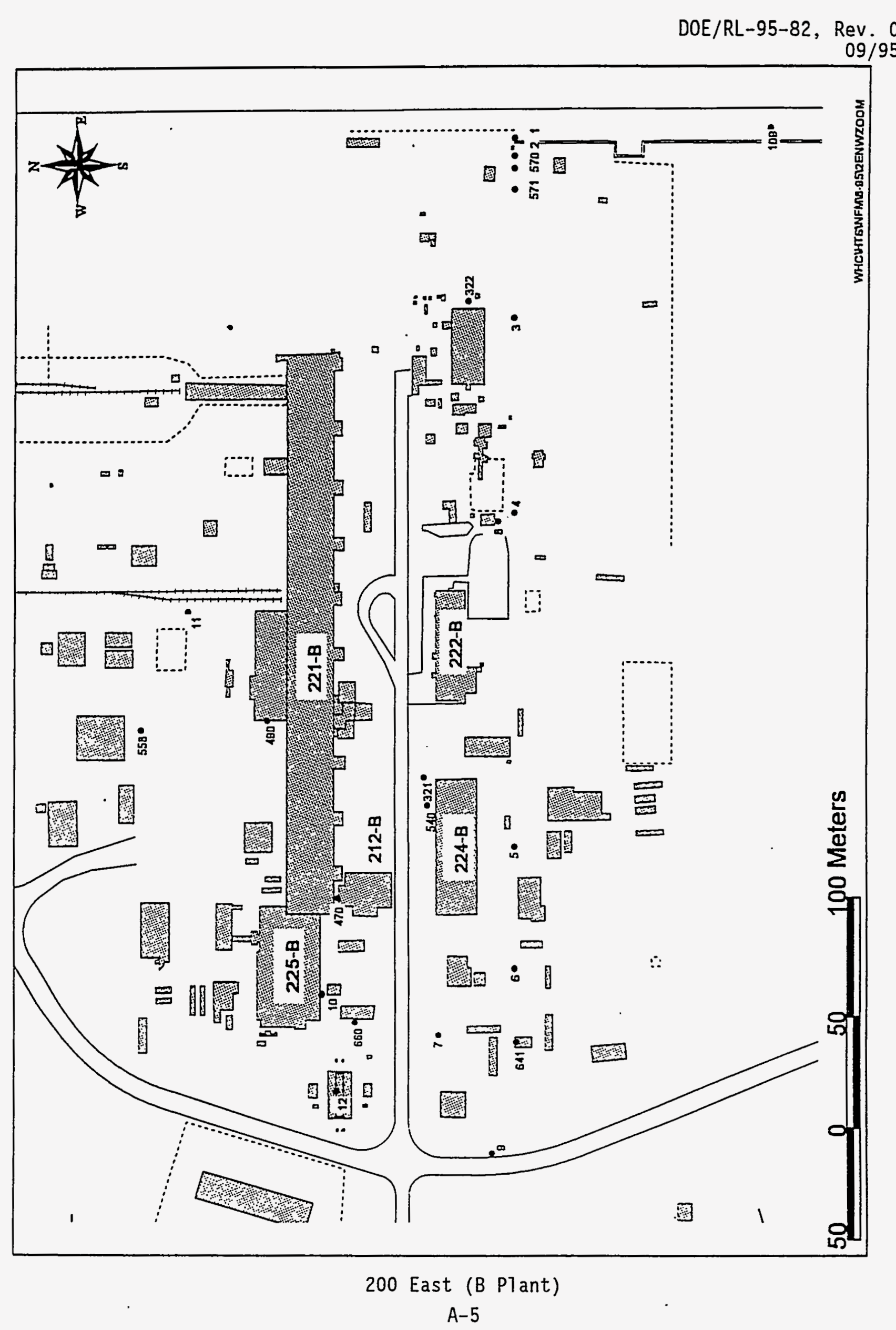




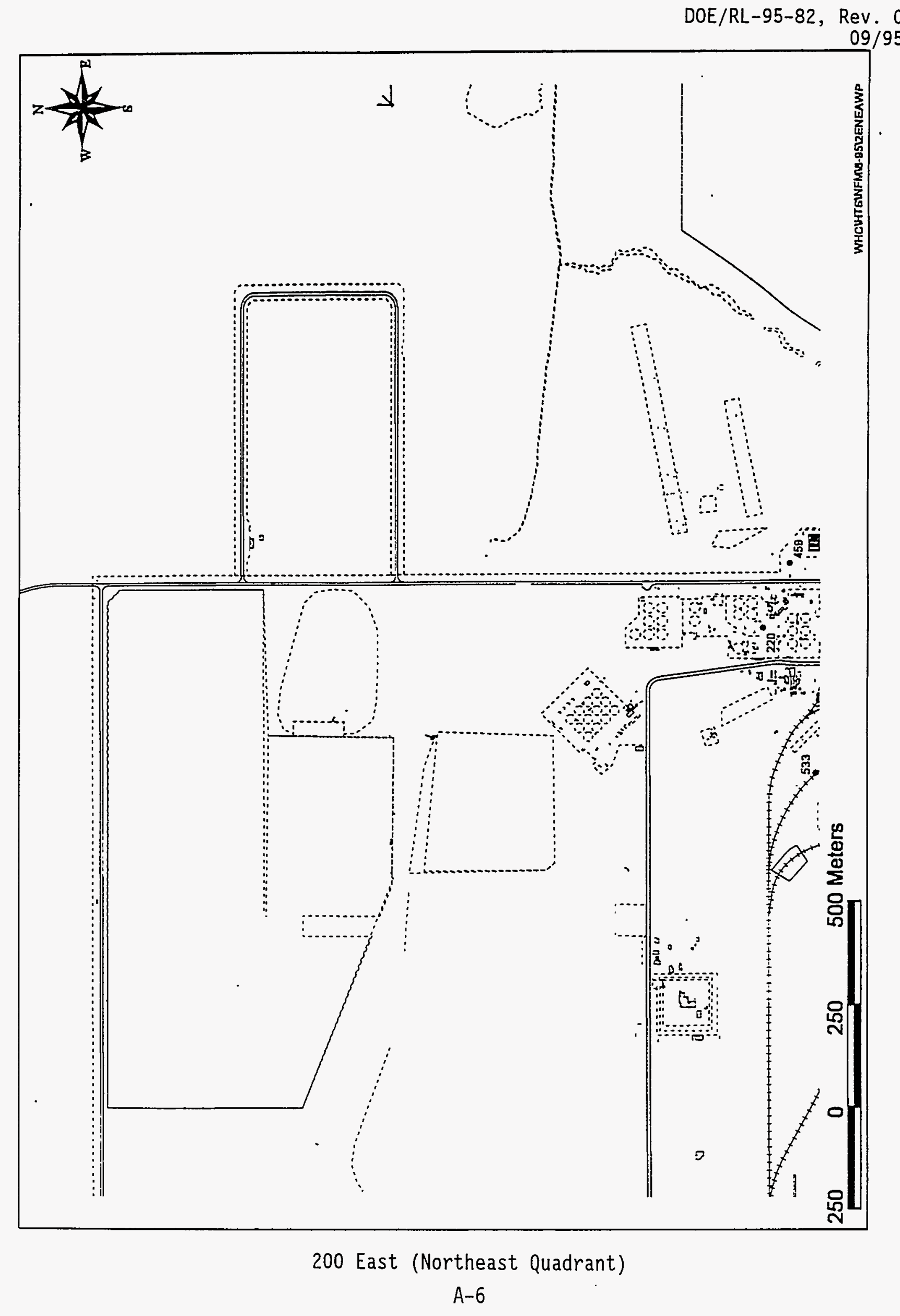


DOE/RL-95-82, Rev. 0

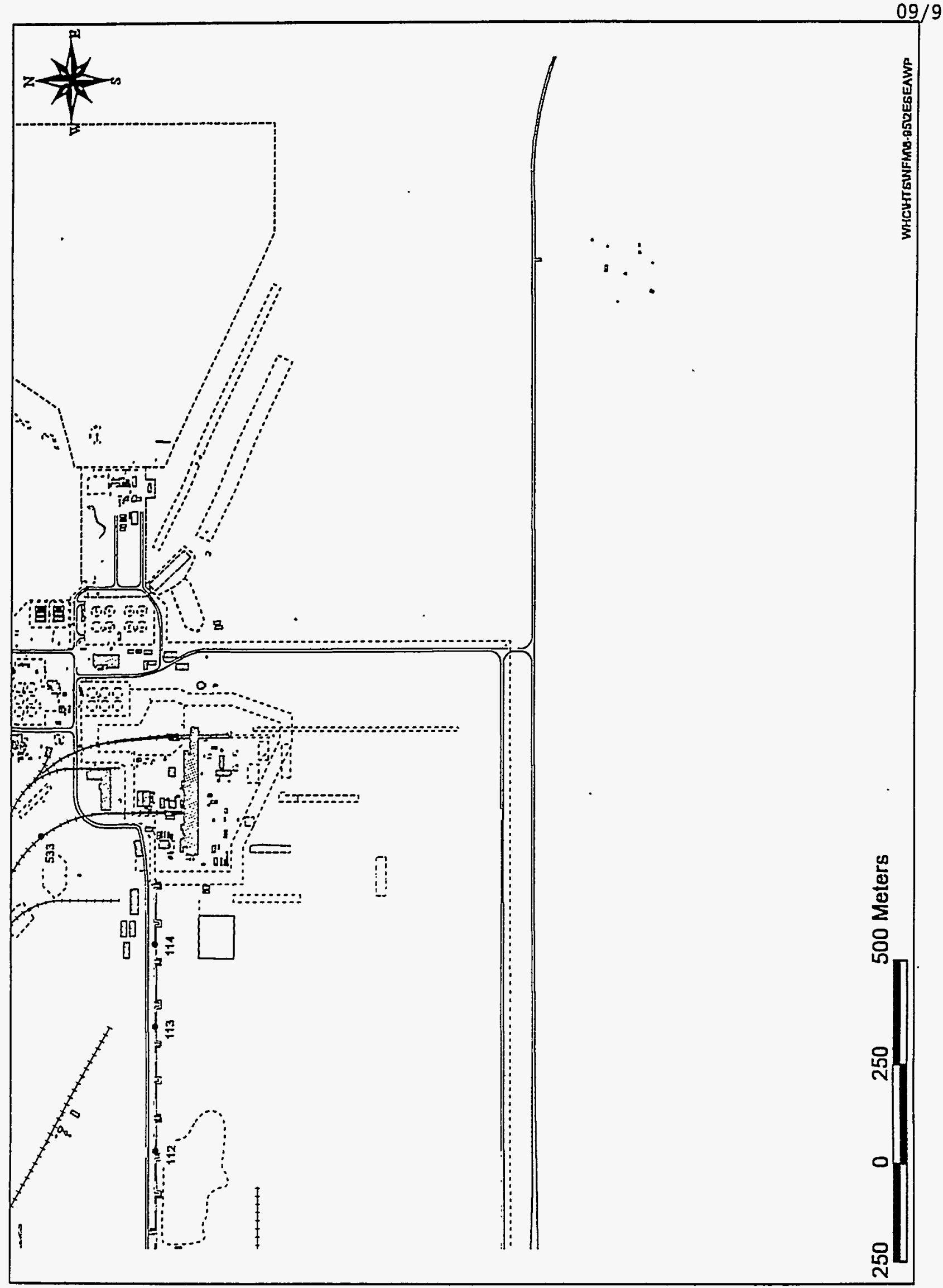

200 East (Southeast Quadrant) 


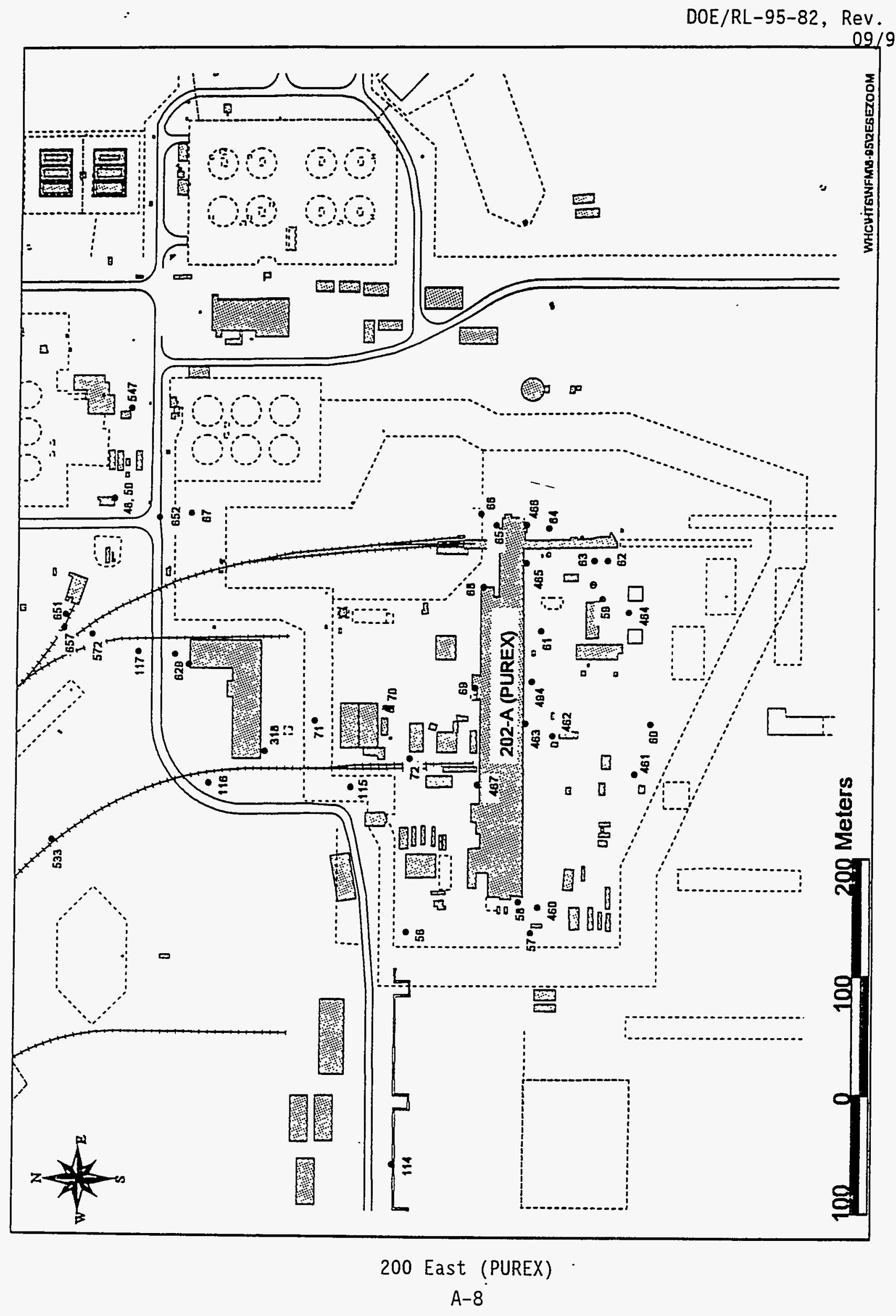




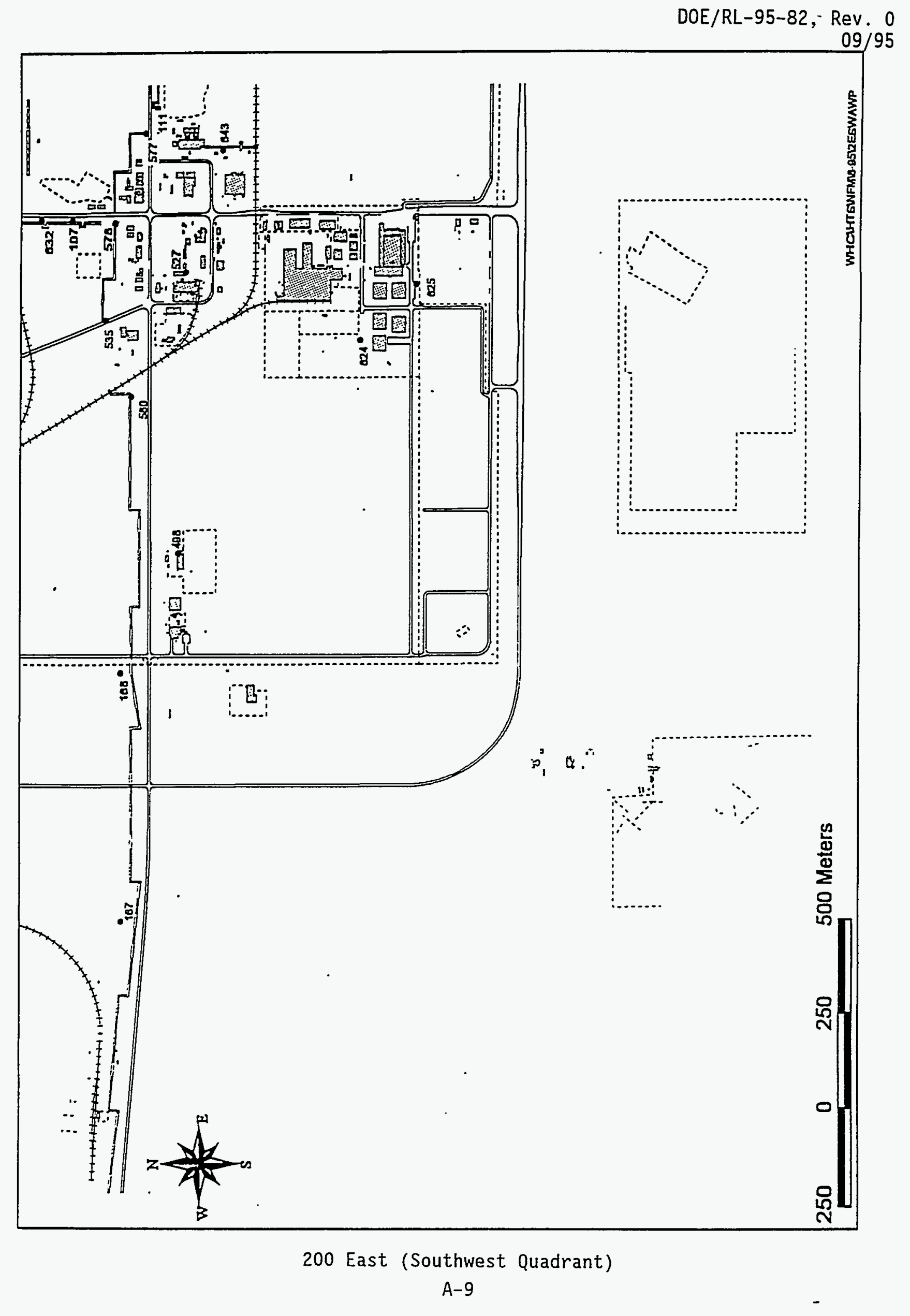




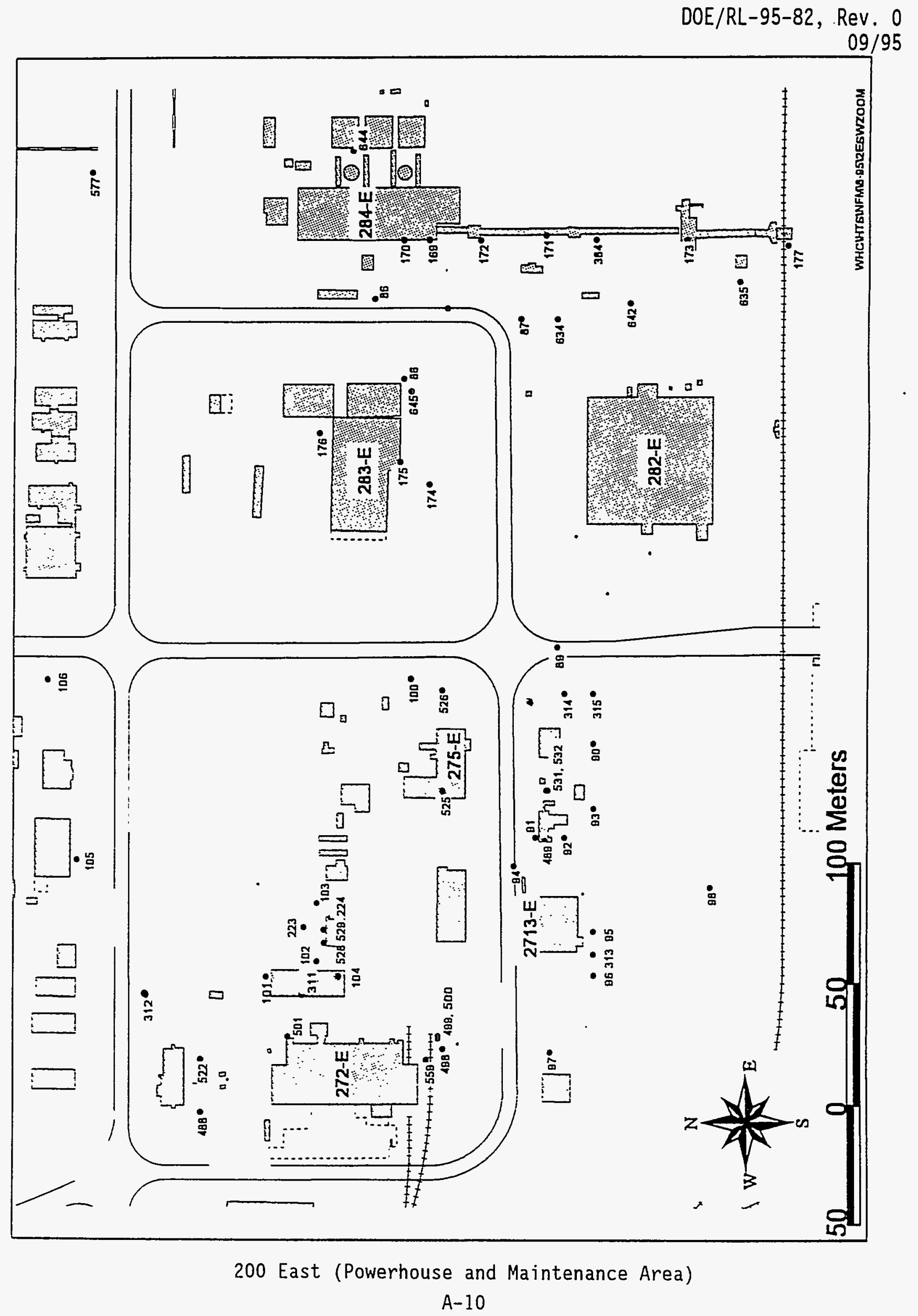




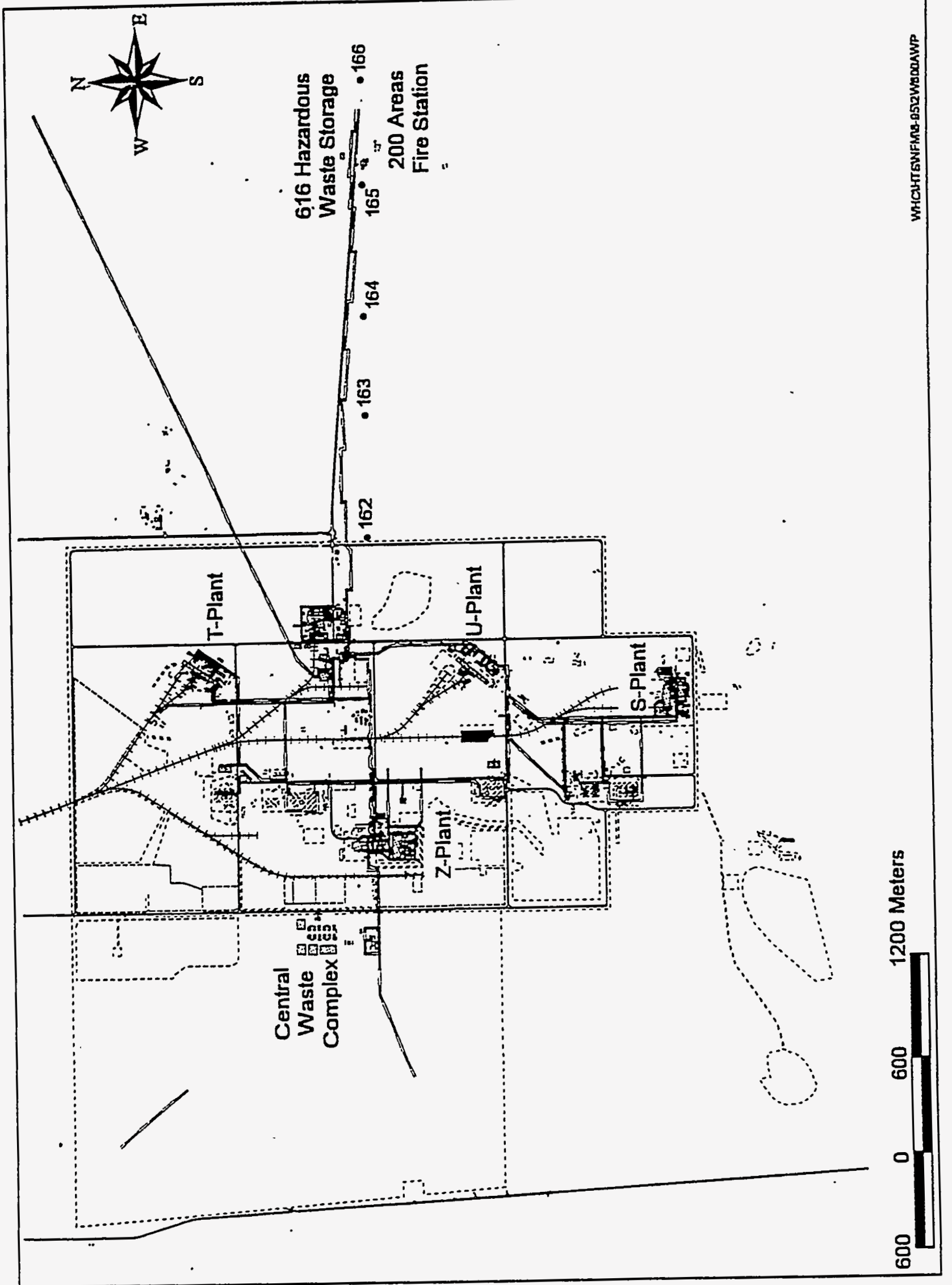

200 West Cross Site Transfer Line 


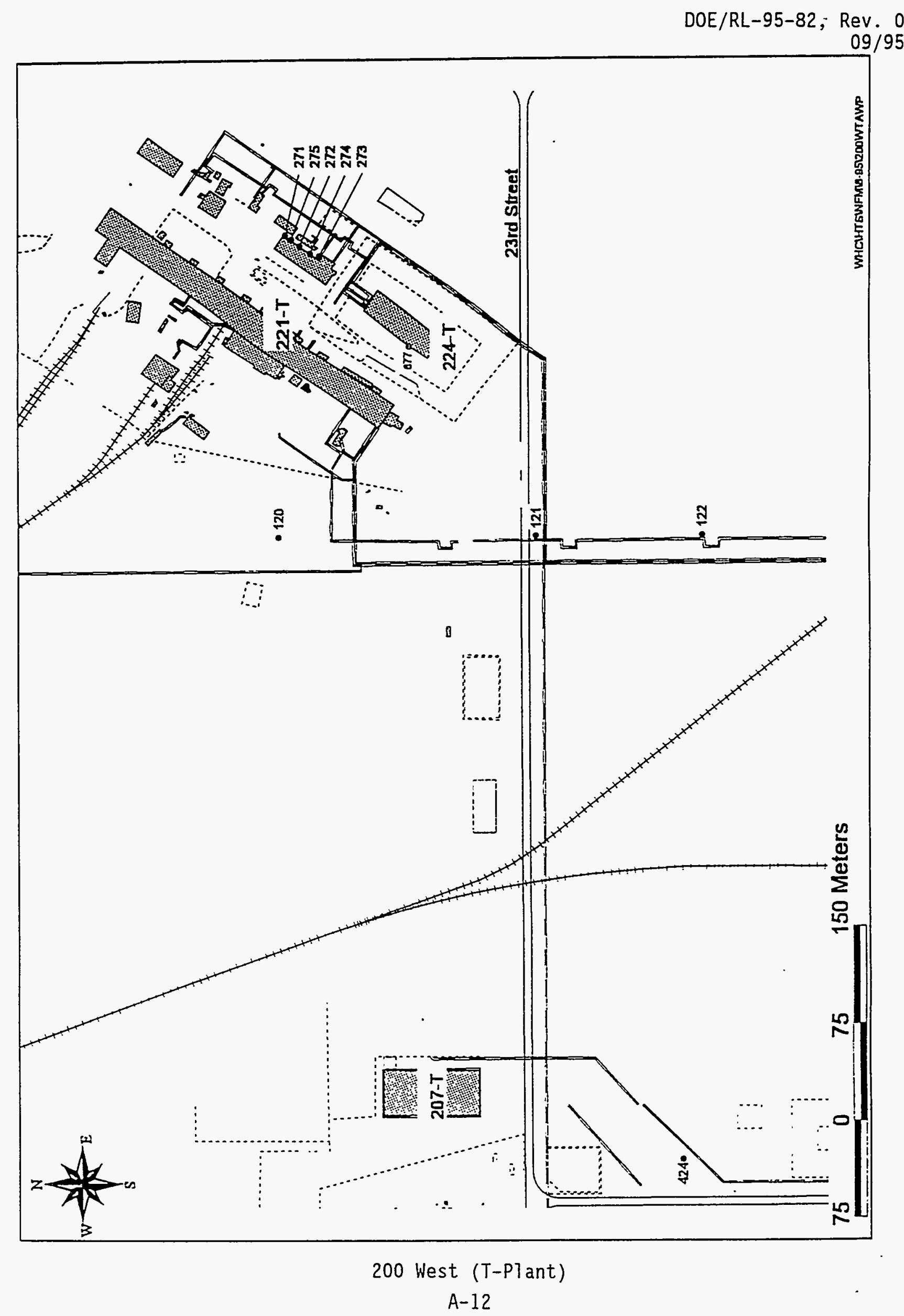




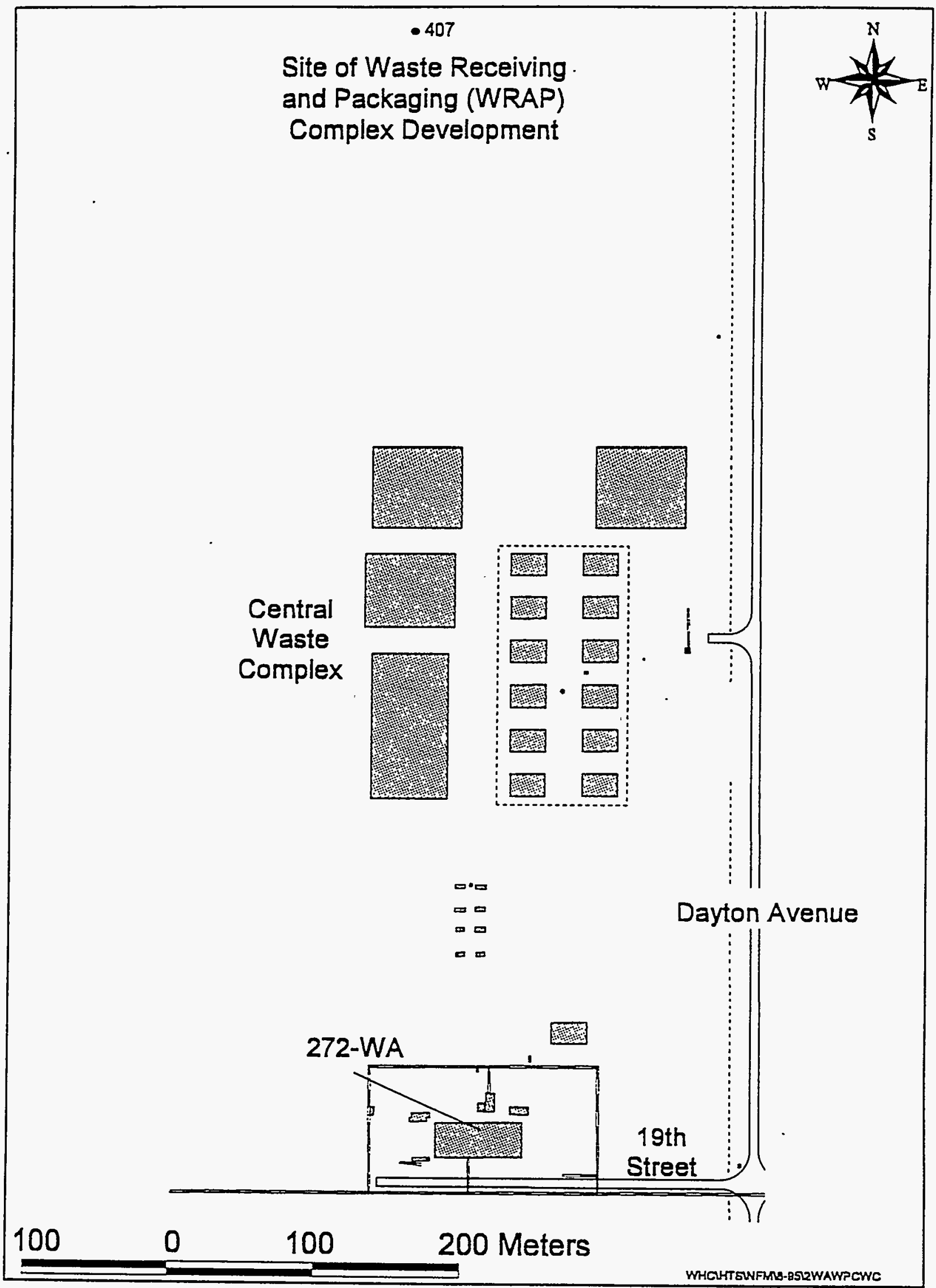

200 West (Central Waste Complex)

A-13 


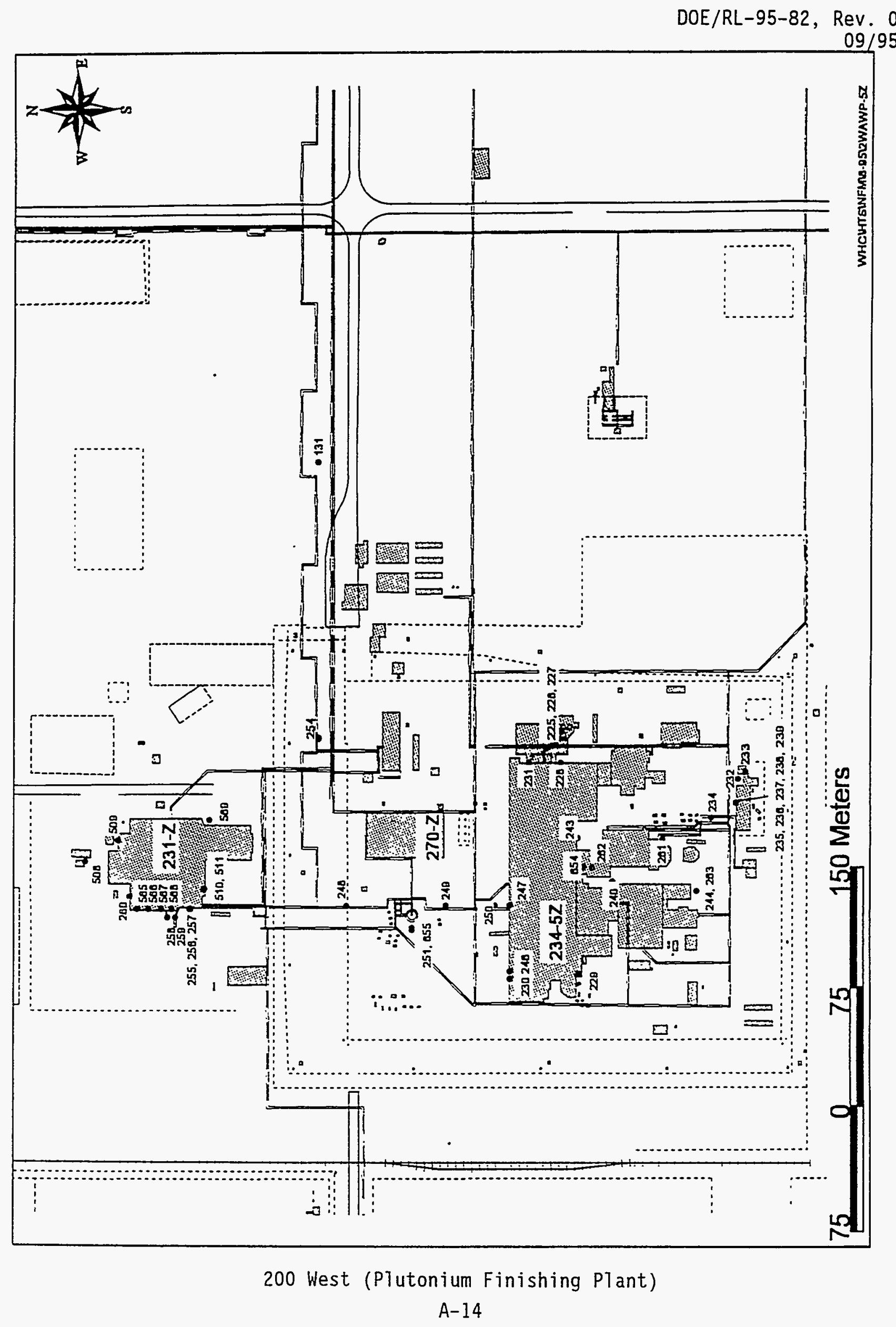




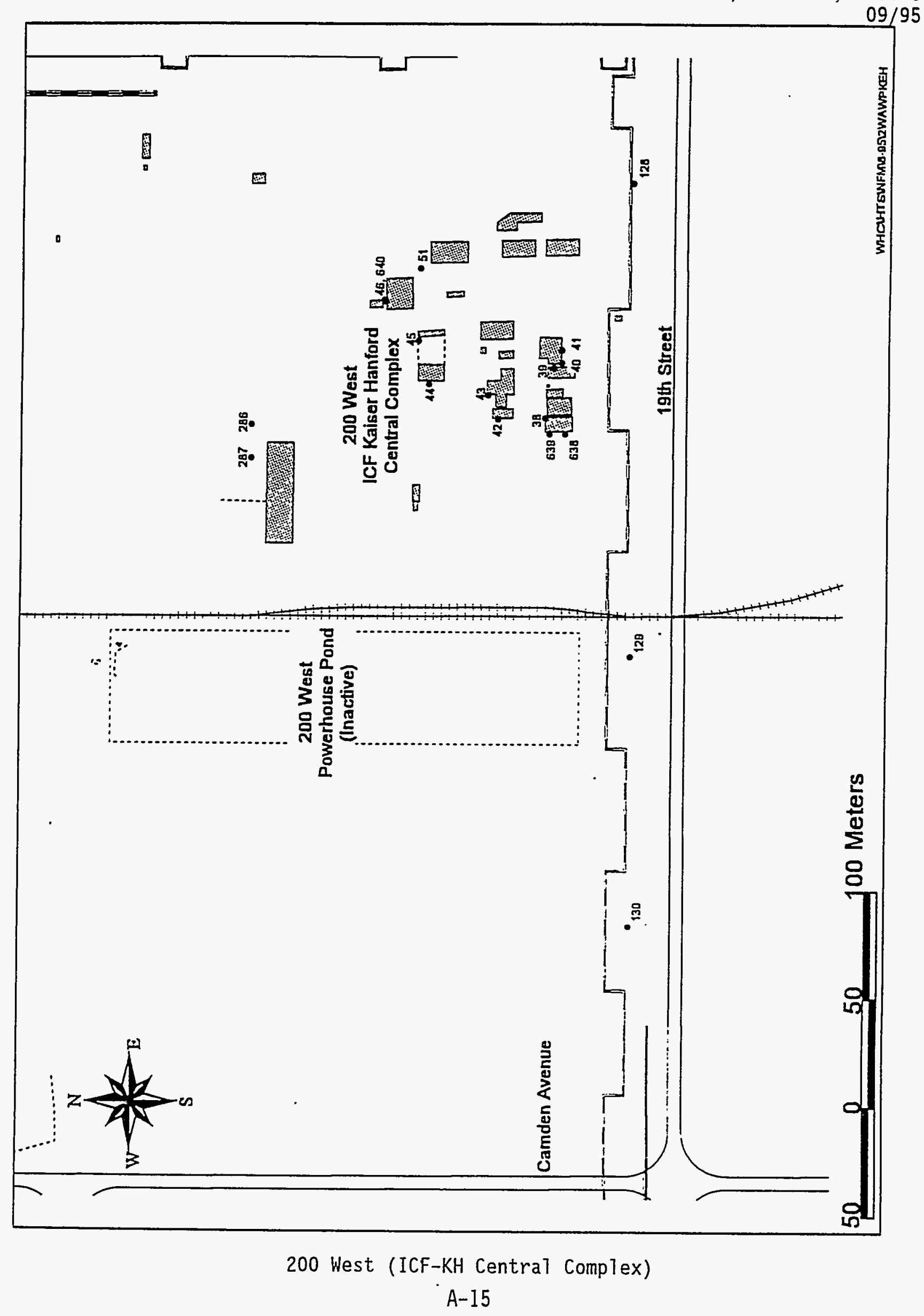


D0E/RL-95-82, Rev. 0 $09 / 95$

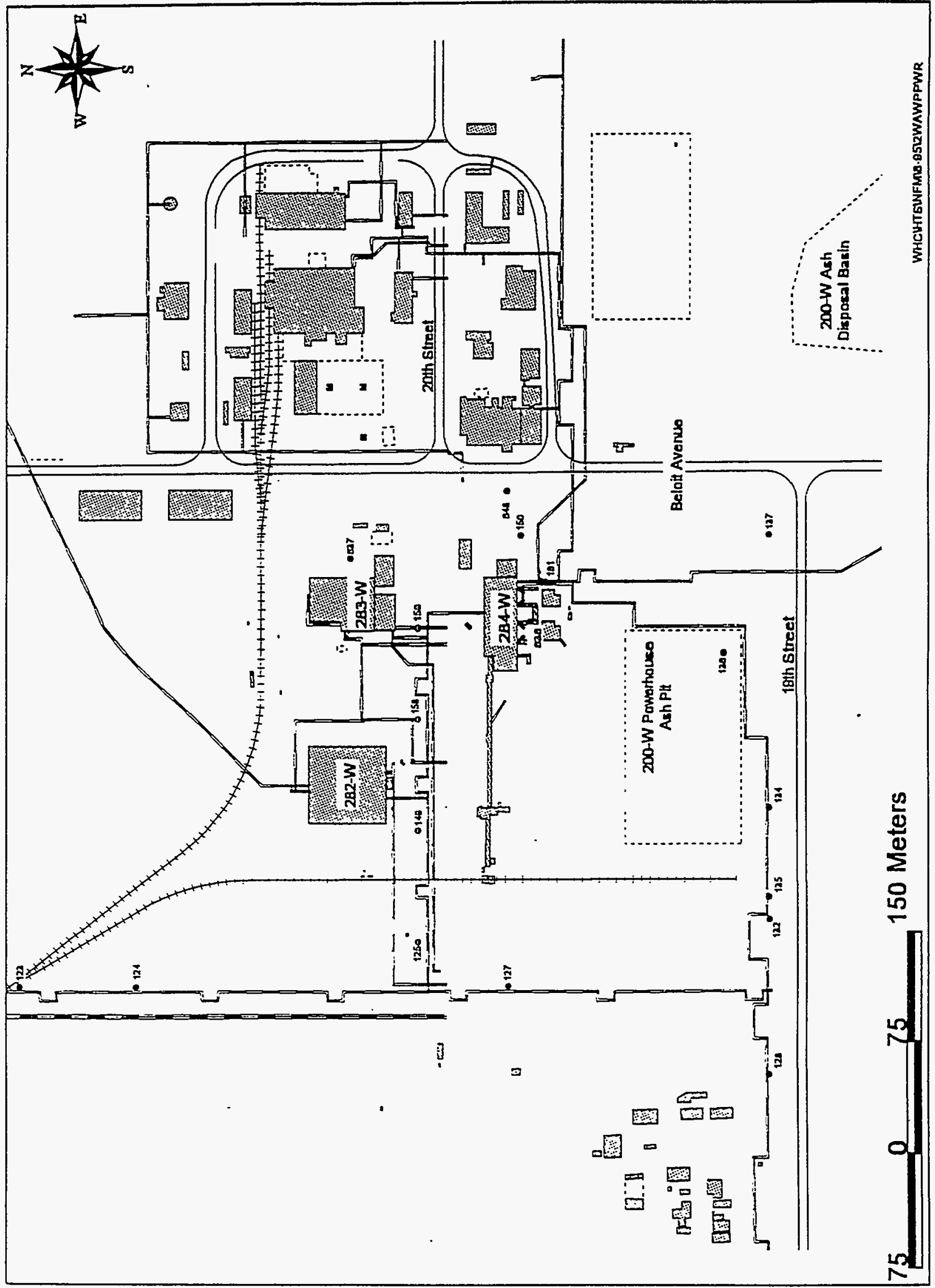

200 West (Powerhouse Area)

A-16 
DOE/RL-95-82, Rev. 0 $09 / 95$

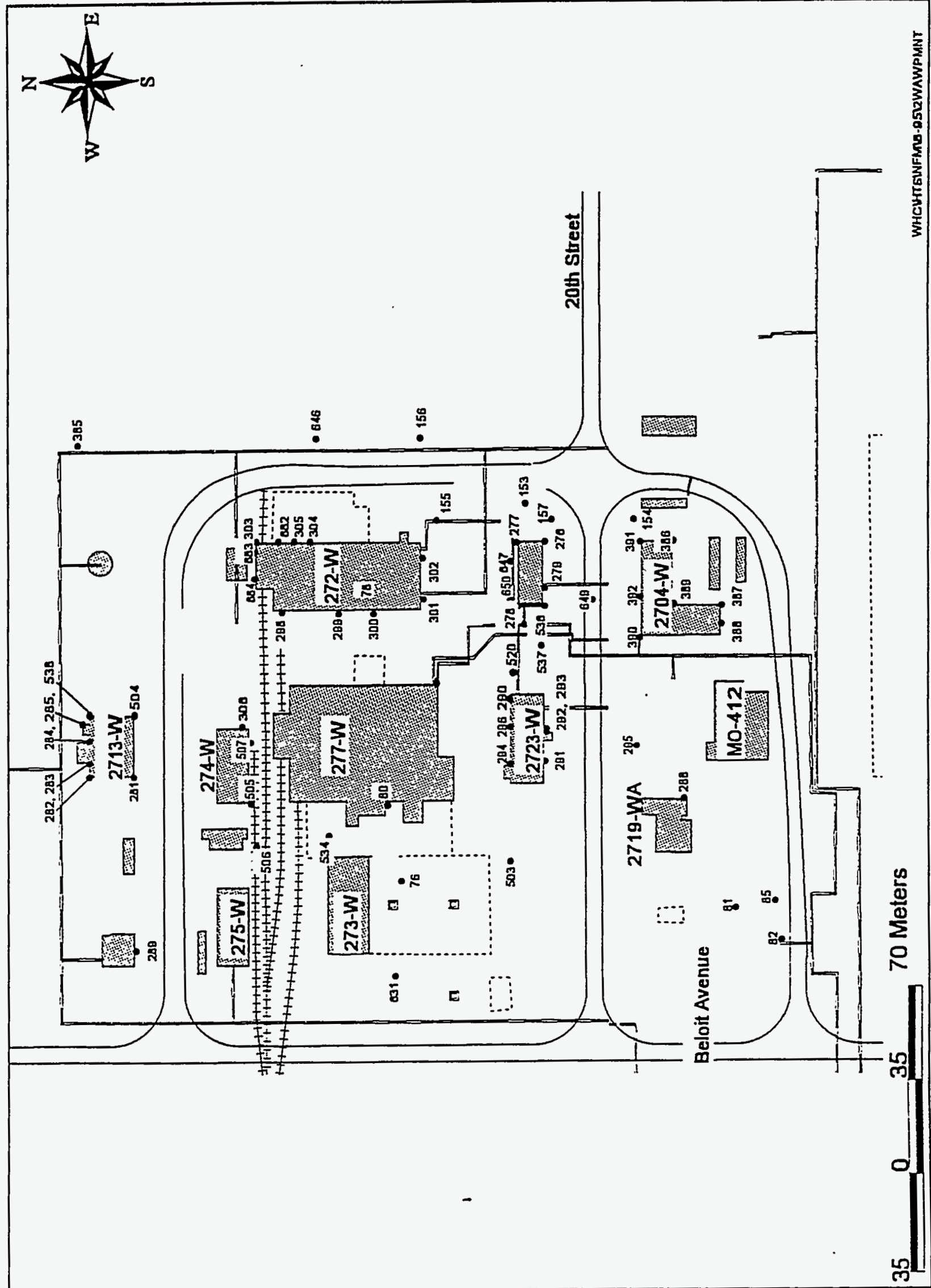

200 West (Maintenance Area) 


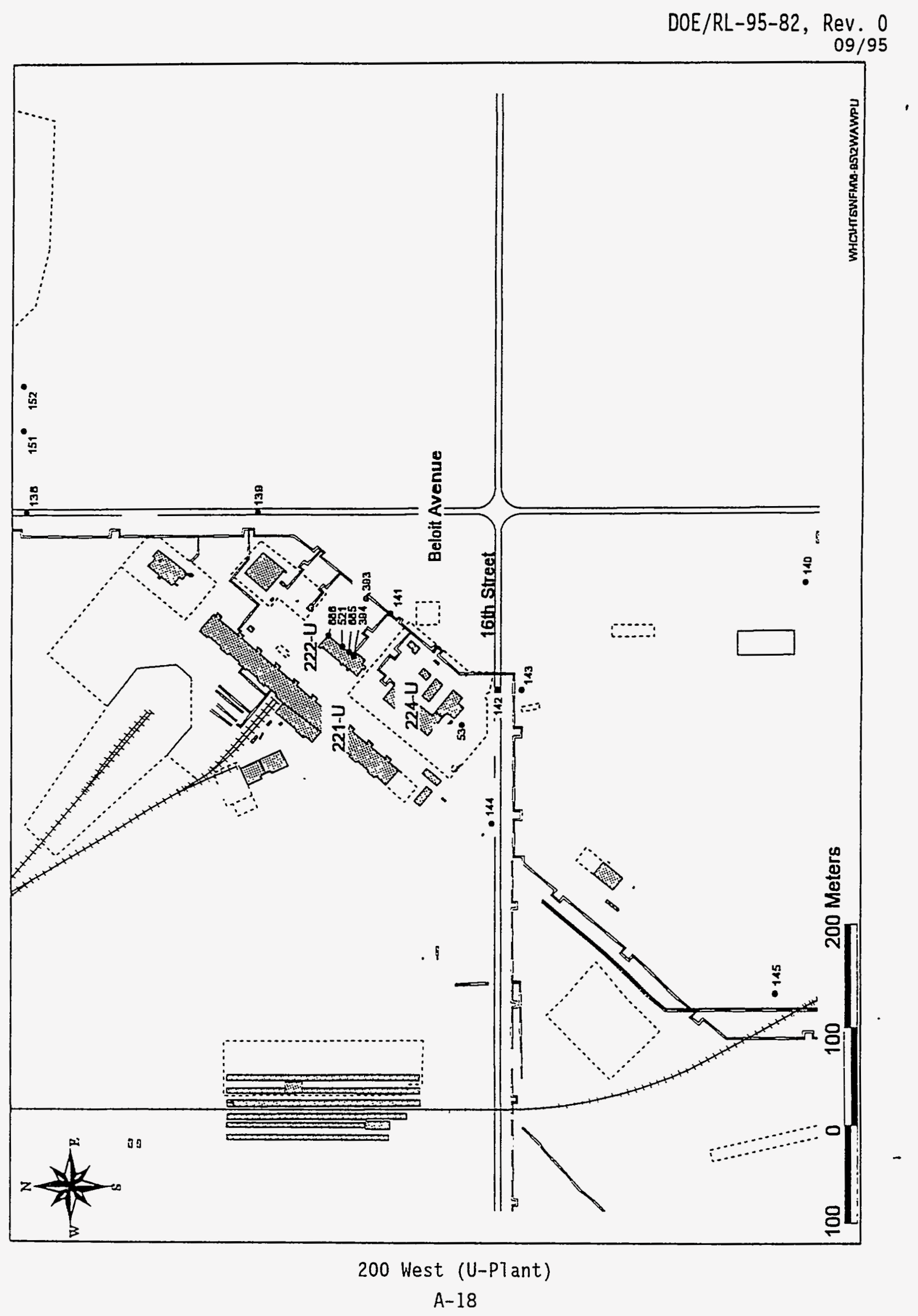


DOE/RL-95-82, Rev. 0

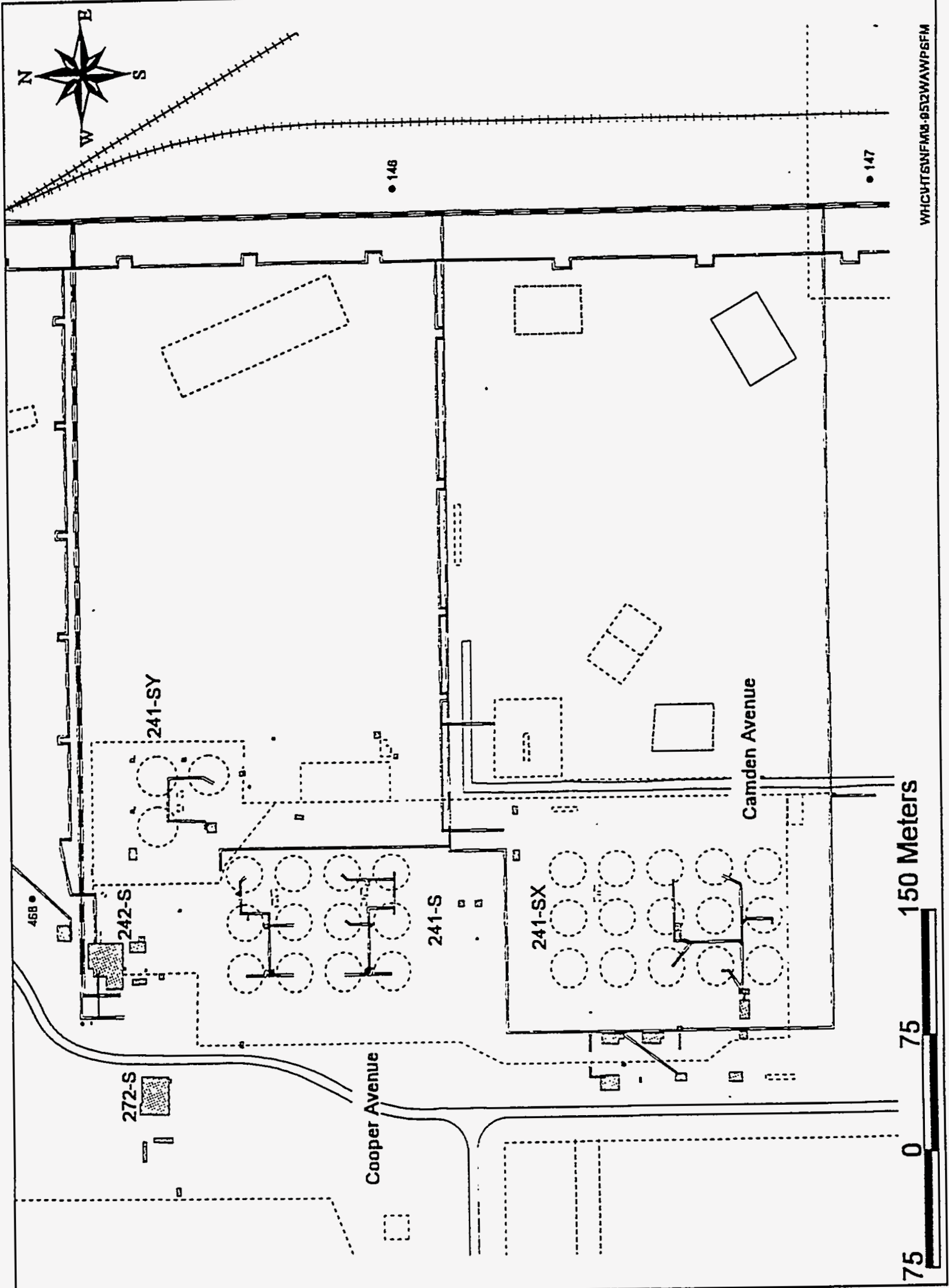

200 West (S-Tank Farm Complex) A-19 
DOE/RL-95-82, Rev. 0 09/95

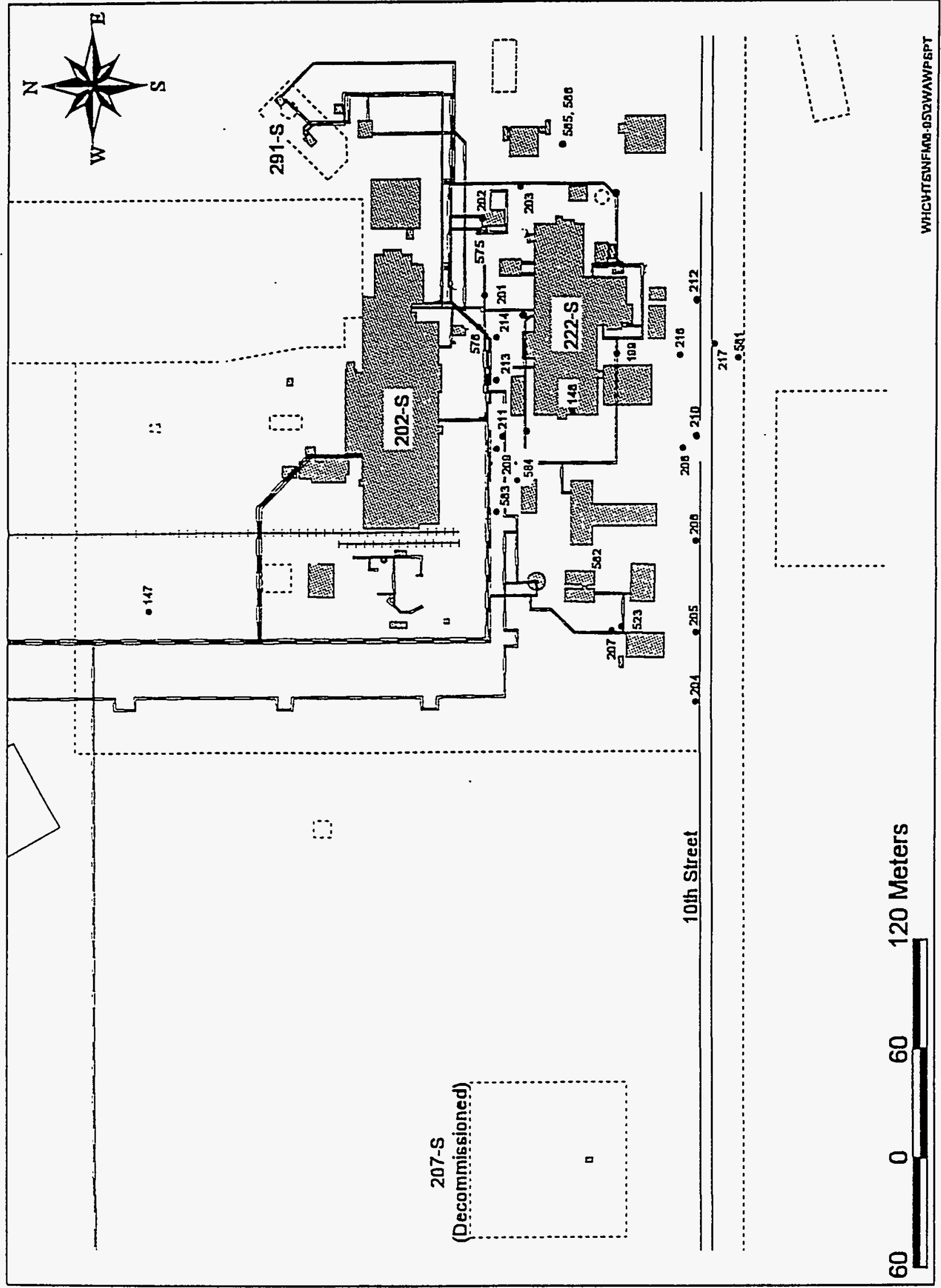

200 West (222-S Laboratory) 


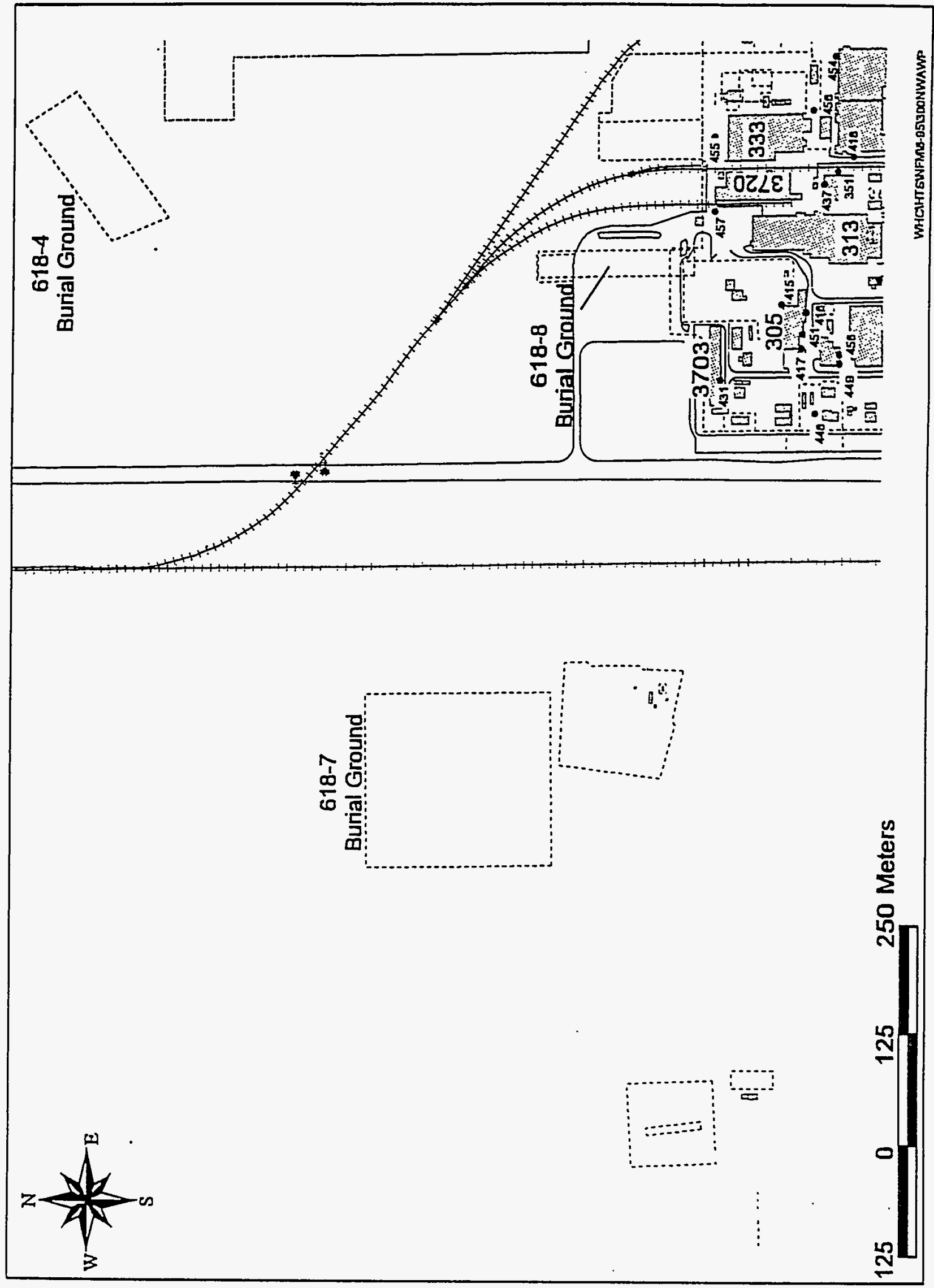




$$
\begin{array}{r}
\mathrm{DOE} / \mathrm{RL}-95-82, \begin{array}{r}
\mathrm{Rev} .0 \\
09 / 95
\end{array}
\end{array}
$$

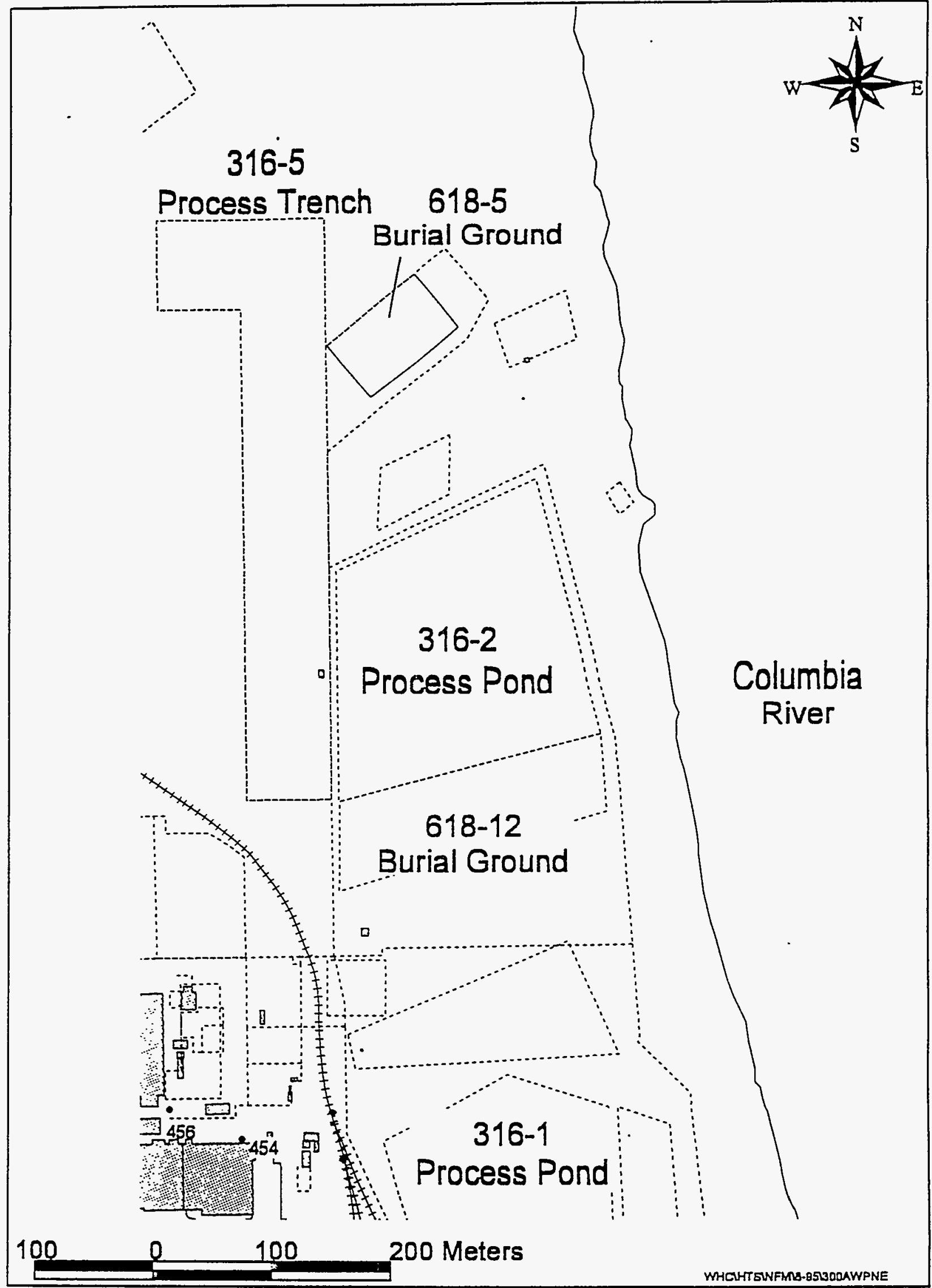

300 Area (Northeast Quadrant) 
DOE/RL-95-82, Rev. 0 $09 / 95$

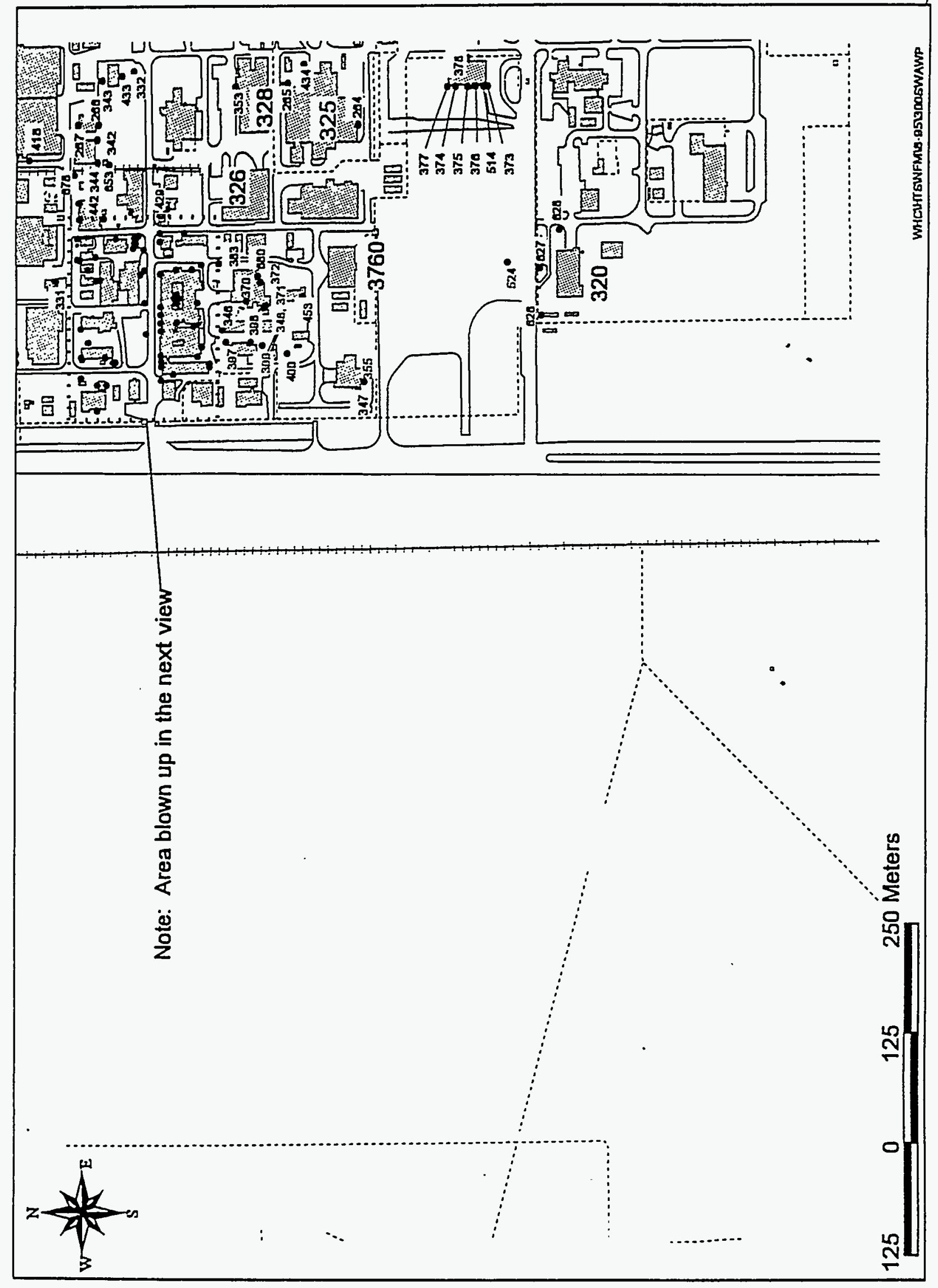

300 Area (Southwest Quadrant) 
DOE/RL-95-82, Rev. 0 $09 / 95$

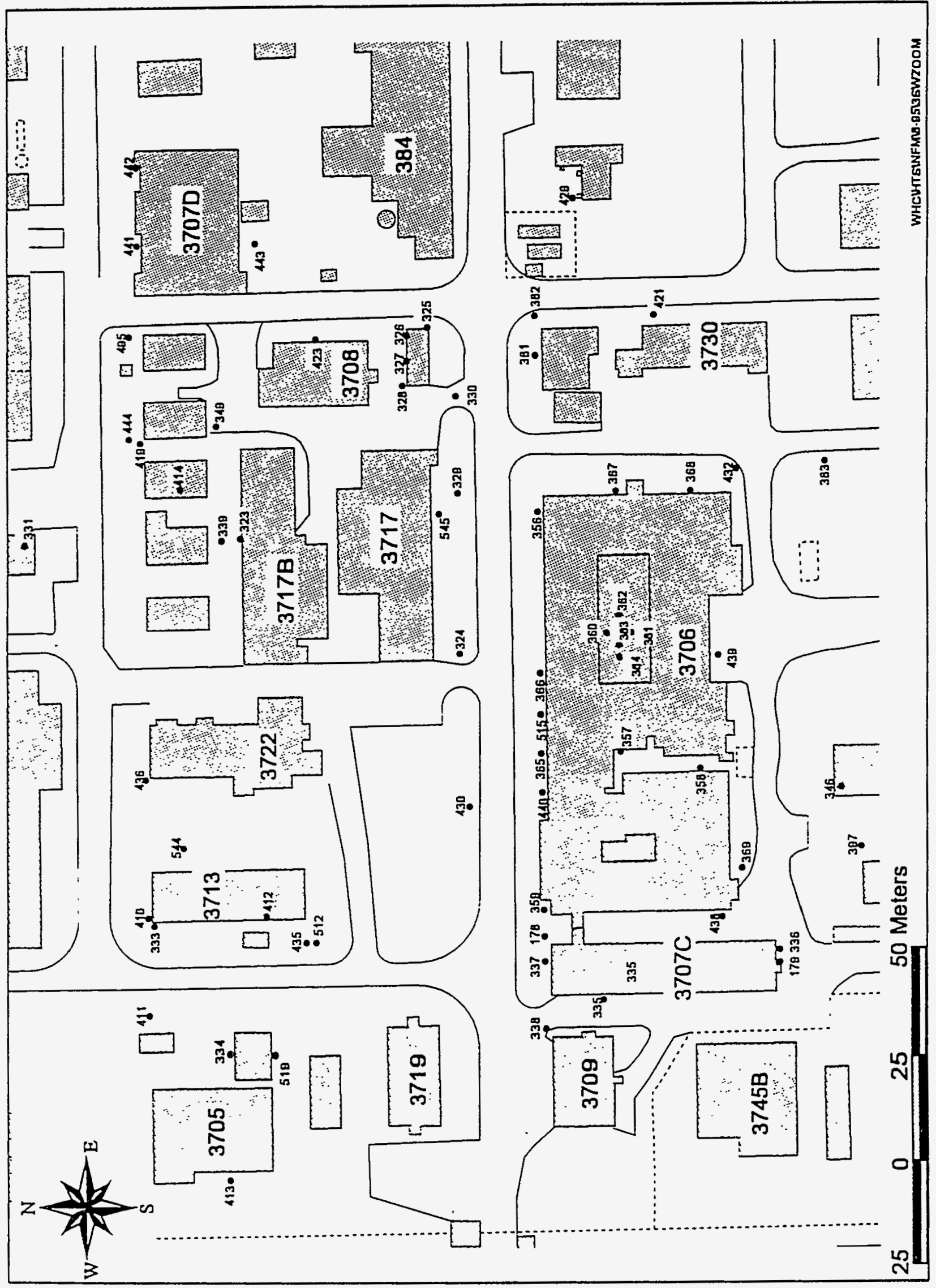

300 Area (3706 Building Area) 
DOE/RL-95-82, Rev. 0

$09 / 95$

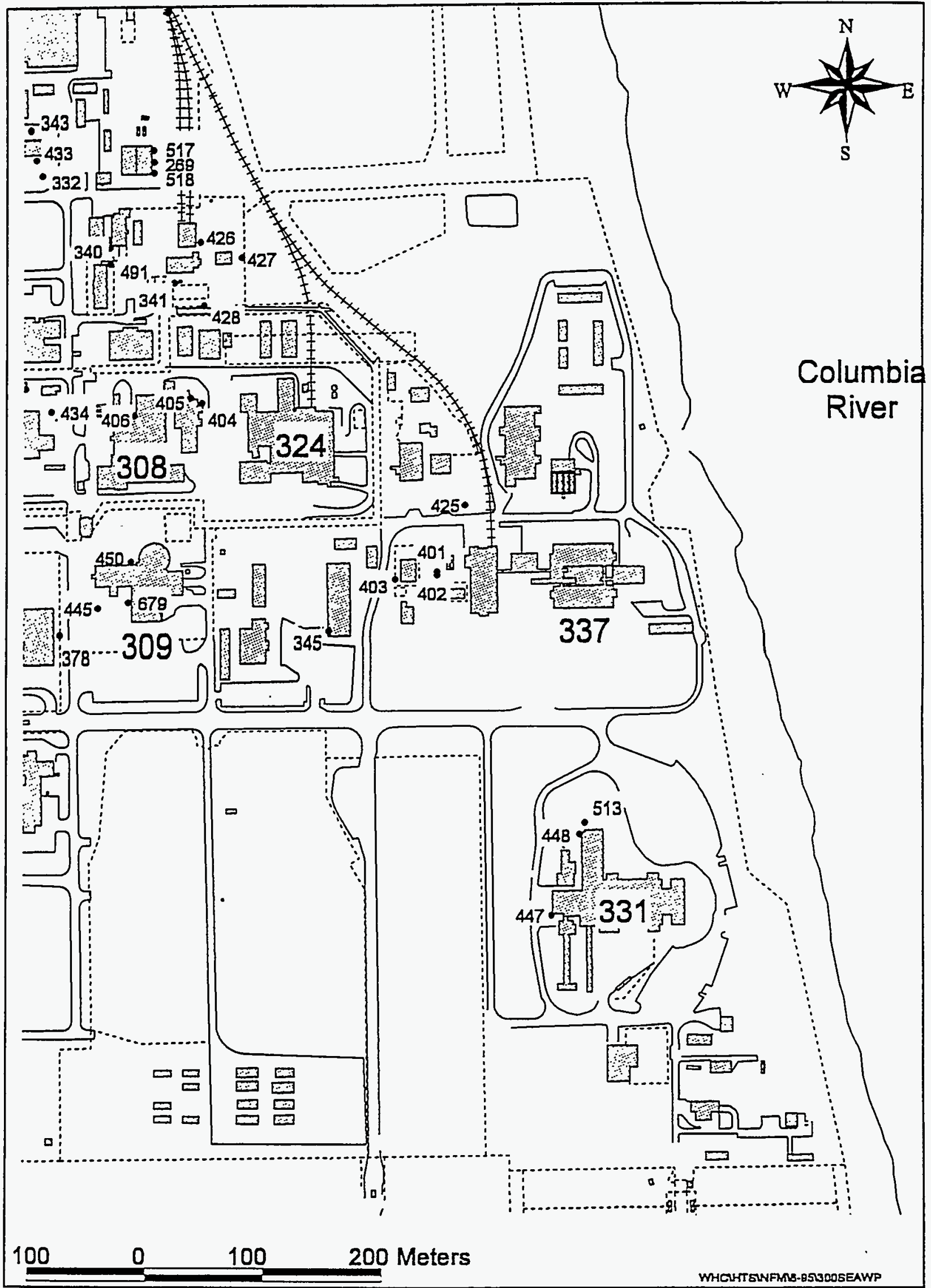

300 Area (Southeast Quadrant) 


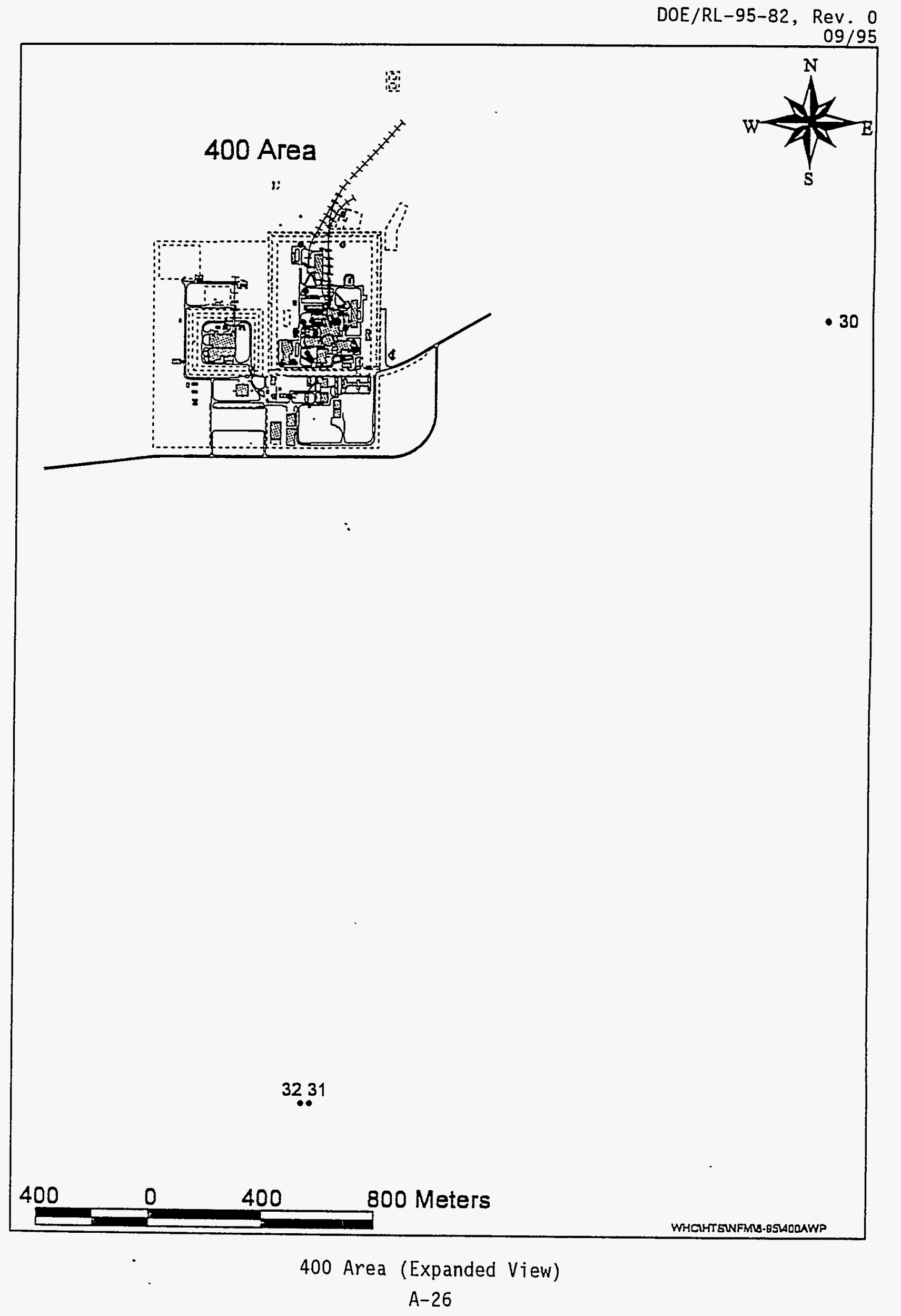




\section{DOE/RL-95-82, Rev. 0}

$=\frac{N}{4}_{3}^{2}$

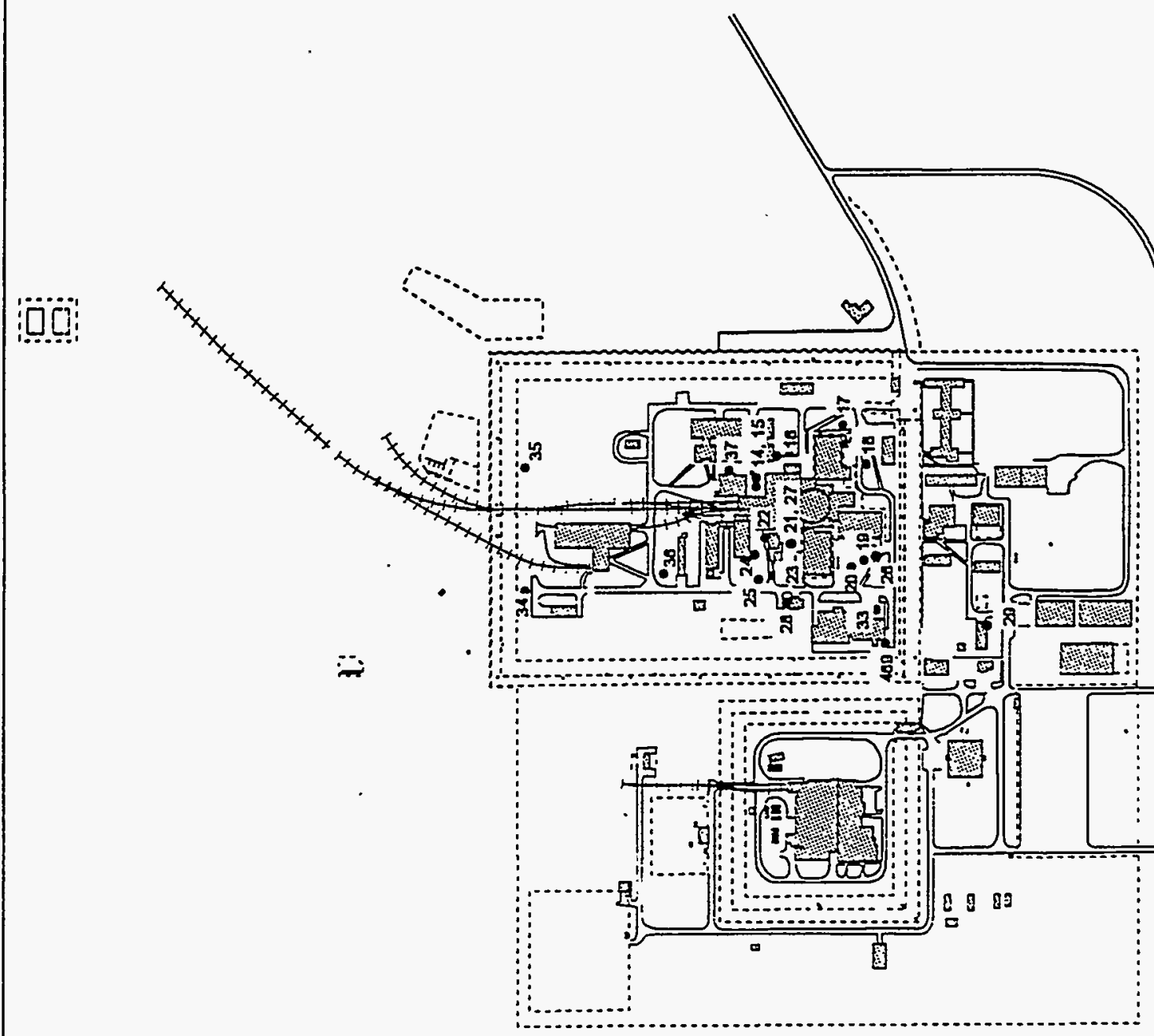


DOE/RL-95-82, Rev. 0

09/95

(This page intentionally left blank) 


\section{DISTRIBUTION}

Number of Copies

OFFSITE

5 Washington State Department of Ecology

1315 W. 4th Avenue

Kennewick, WA 99336-6018

Melodie A. Selby (2)

David S. Dougherty

Michael Hepp

1 U.S. Environmental Protection Agency

Doug Sherwood - EPA Region 10

712 Swift Boulevard, Suite 5

Richland, WA 99352

\section{ONSITE}

19 U.S. Department of Energy,

Richland Operations Office

MSIN

M. A. Barnard

LA-40

E. M. Bowers

S7-55

R. P. Carter

S7-55

D. H. Chapin

N2-36

B. A. Davis

R3-82

D. T. Evans

S7-41

R. N. Krekal

A5-15

D. J. Ortiz

G3-18

P. M. Pak

H4-83

J. E. Rasmussen

A5-15

L. D. Romine

R3-79

W. A. Rutherford

A5-51

C. O. Ruud

S7-54

W. D. Seaborg

T5-50

G. L. Sinton

S7-55

H. R. Trumble

H4-83

W. A. White

G3-18

Reading Room (2)

H2-53

5 Betchel Hanford Incorporated

J. H. Dunkirk

H4-86

R. J. Landon

H4-86

L. A. Mihalik

H4-79

J. E. Rugg

H4-86 


\section{DISTRUBUTION}

11 ICF-Kaiser Hanford

T. D. Boucher $\quad S 2-41$

B. J. Dixon (2) B4-20

M. R. Gunter B4-20

R. G. Hollenbeck G3-17

D. R. Herman $\quad$ S2-12

D. L. Klages $\$ 2-51$

C. E. Marple $\quad$ S2-51

R. L. Newell B4-20

A. D. Poor L6-55

$4 \quad$ Pacific Northwest Laboratory

D. S. Broussard K1-67

T. J. Lazarski P7-79

$\begin{array}{ll}\text { R. K. Woodruff } & \text { P7-68 }\end{array}$

Hanford Technical Library K1-11

28 Westinghouse Hanford Company

B. P. Atencio H6-25

L. E. Bomeman . R2-06

P. J. Crane T3-38

P. J. Davis-Vedder H6-22

B. G. Erlandson H6-20

D. L. Flyckt T7-38

C. J. Geier R2-36

R. D. Gustavson R1-51

A. J. Diliberto H6-32

L. P. Diediker T1-30

W. T. Dixon H6-21

M. J. Hall T6-14

S. E. Killoy $\quad$ S6-70

C. J. Lewis T3-01

J. J. Luke H6-25

K. J. Lueck H6-28

D. J. McBride T5-54

A. K. McDowell R1-51

P. C. Miller N2-33

B. D. Williamson B3-06

J. D. Williams H6-28

D. W. Wilson $\quad$ S6-30

C. D. Wollam $\quad$ S6-17

Central Files (1) A3-08

Document Processing and Distribution (2) L8-15

OSTI (2) A3-36 